\title{
Planejamento sob incerteza para metas de alcançabilidade estendidas
}

\author{
Silvio do Lago Pereira
}

\author{
TESE APRESENTADA \\ AO \\ Instituto de MATEMÁticA E EstatísticA \\ DA \\ UNIVERSIDADE DE SÃO PAULO \\ PARA \\ ObTEnÇÃo Do Título \\ $\mathrm{DE}$ \\ DOUTOR EM CIÊNCIAS
}

\begin{abstract}
Área de concentração: Ciência da Computação
Orientadora: $\operatorname{Prof}^{a}{ }^{a} \operatorname{Dr}^{a}$. Leliane Nunes de Barros
\end{abstract}

— São Paulo, novembro de 2007 _ 



\title{
Planejamento sob incerteza para metas de alcançabilidade estendidas
}

\author{
Este exemplar corresponde à redação \\ final da tese, devidamente corrigida e \\ defendida por Silvio do Lago Pereira, \\ aprovada pela comissão julgadora.
}

São Paulo, novembro de 2007.

Banca examinadora:

- Prof ${ }^{a}$. Dr. ${ }^{a}$ Leliane Nunes de Barros (presidente) - IME-USP

- Prof. Dr. Marcelo Finger - IME-USP

- Prof. Dr. Fábio Gagliard Cozman - EP-USP

- Prof. Dr. Jacques Wainer - IC-UNICAMP

- Prof. Dr. Marcos Alexandre Castilho - DI-UFPR

Suplentes:

- Prof. ${ }^{a}$ Dr. ${ }^{a}$ Renata Wassermann - IME-USP

- Prof. Dr. Jaime Simão Sichman - EP-USP 

A duas pessoas que não puderam ver a conclusão desse trabalho:

Joaquim, mais que um pai, $e$ Itivaldo, mais que um irmão. 



\section{Agradecimentos}

Durante os cinco anos em que me dediquei a esse doutorado, tive a felicidade de contar com o apoio de muitas pessoas. Agradeço, sinceramente, a cada uma delas!

À professora Leliane Nunes de Barros, pela dedicação e paciência com que me orientou, sempre contribuindo com idéias muito valiosas para melhorar cada parte dessa tese. Mais que orientadora, considero-a uma amiga muito estimada, que sempre demonstrou grande interesse pelo meu crescimento como pesquisador.

Ao professor Fábio Gagliardi Cozman da EP-USP, com quem tivemos a oportunidade de discutir alguns dos resultados que apresentamos no Capítulo 6 dessa tese.

Aos professores do Departamento de Ciência da Computação do IME-USP, que contribuíram para a minha formação nesse doutorado. Especialmente aos professores Marcelo Finger e Ana Cristina Vieira de Melo que, como membros da banca no meu exame de qualificação de doutorado, fizeram observações bastante úteis para a definição do escopo dessa tese.

Aos colegas do Departamento de Processamento de Dados da FATECSP-UNESP, que sempre me apoiaram e me incentivaram a prosseguir com meus estudos. Especialmente ao professor Luiz Tsutomu Akamine, pelas inúmeras horas que passamos conversando sobre assuntos relativos ao meu doutorado.

A todos meus amigos, que encararam os meus momentos de mau humor (que não foram raros nesses últimos meses) com muito bom humor. Especialmente à Cláudia Lello Courtouké, ao Leandro César Prudente e ao Fred Oliver.

À minha mãe, Maria José do Lago Pereira, e a toda a minha família que, apesar do meu distanciamento no período em que estive dedicado a esse trabalho, sempre estiveram comigo nos momentos em que eu mais precisei.

Finalmente, agradeço a Deus, que me permitiu encontrar todas essas pessoas ao longo do meu caminho... Se existe mesmo destino, acho que Deus planejou o meu nesse doutorado com base na meta $\forall \bullet \exists($ encontrar_pessoas_maravilhosas $\sqcup$ doutorado_concluído $)$. 



\section{Resumo}

Planejamento sob incerteza vem sendo cada vez mais requisitado em aplicações práticas de diversas áreas que requerem soluções confiáveis para metas complexas. Em vista disso, nos últimos anos, algumas abordagens baseadas no uso de métodos formais para síntese automática de planos têm sido propostas na área de Planejamento em Inteligência Artificial. Entre essas abordagens, planejamento baseado em verificação de modelos tem se mostrado uma opção bastante promissora; entretanto, conforme observamos, a maioria dos trabalhos dentro dessa abordagem baseiase em CTL e trata apenas problemas de planejamento para metas de alcançabilidade simples (como aquelas consideradas no planejamento clássico). Nessa tese, introduzimos uma classe de metas de planejamento mais expressivas (metas de alcançabilidade estendidas) e mostramos que, para essa classe de metas, a semântica de CTL não é adequada para formalizar algoritmos de síntese (ou validação) de planos. Como forma de contornar essa limitação, propomos uma nova versão de CTL, que denominamos $\alpha$-CTL. Então, a partir da semântica dessa nova lógica, implementamos um verificador de modelos (VACTL), com base no qual implementamos também um planejador (PACTL) capaz de resolver problemas de planejamento para metas de alcançabilidade estendidas, em ambientes não-determinísticos com observabilidade completa. Finalmente, discutimos como garantir a qualidade das soluções quando dispomos de um modelo de ambiente onde as probabilidades das transições causadas pela execução das ações são conhecidas. 



\begin{abstract}
Planning under uncertainty has being increasingly demanded for practical applications in several areas that require reliable solutions for complex goals. In sight of this, in the last few years, some approaches based on formal methods for automatic synthesis of plans have been proposed in the area of Planning in Artificial Intelligence. Among these approaches, planning based on model checking seems to be a very attractive one; however, as we observe, the majority of the works in this approach are mainly based on CTL and deals only with planning problems for simple reachability goals (as those considered in classical planning). In this thesis, we introduce a more expressive class of planning goals (extended reachability goals) and show that, for this class of goals, the CTL's semantics is not adequate to formalize algorithms for synthesis (or validation) of plans. As a way to overcome this limitation, we propose a new version of CTL, called $\alpha$-CTL. Then, based on the semantics of this new logic, we implement a model checker (VACTL), based on which we also implement a planner (PACTL) capable of solving planning problems for extended reachability goals, in nondeterministic planning environments with complete observability. Finally, we discuss how to guarantee the quality of the solutions when we have an environment model where the actions transitions probabilities are known.
\end{abstract}





\section{Sumário}

1 Introdução 1

1.1 O problema de planejamento $\ldots \ldots \ldots \ldots \ldots \ldots$

1.1.1 Planejamento clássico . . . . . . . . . . . . . . . . . 1

1.1.2 Planejamento sob incerteza . . . . . . . . . . . . . . . . . . 4

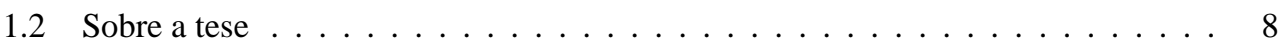

1.2.1 Motivação . . . . . . . . . . . . . . . . . . 9

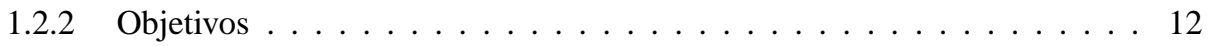

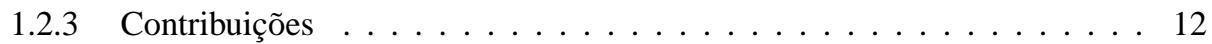

1.3 Organização . . . . . . . . . . . . . . . . . . . . . . . . 13

2 Verificação de modelos $\quad 15$

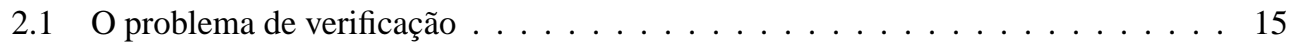

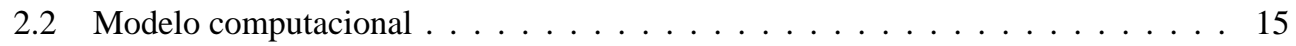

2.2.1 Estruturas de Kripke . . . . . . . . . . . . . . . . . . . . . . . . . 16

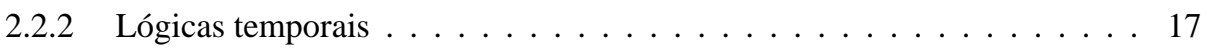

2.3 Verificação baseada em CTL . . . . . . . . . . . . . . . . . . . 22

2.3.1 Caracterização de ponto-fixo . . . . . . . . . . . . . . . . . 22

2.3.2 Computação de pontos-fixos . . . . . . . . . . . . . 23

2.4 Verificação de modelos simbólicos . . . . . . . . . . . . . . 26

2.4.1 Codificação proposicional . . . . . . . . . . . . . . . . . . 27

2.4.2 Diagramas de decisão binária $\ldots \ldots \ldots \ldots$ 
2.4.3 Algoritmo para verificação de modelos simbólicos . . . . . . . . . . 32

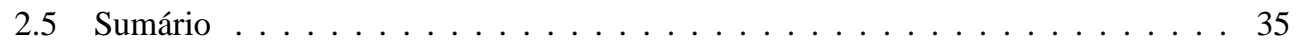

3 Planejamento baseado em verificação de modelos $\quad 37$

$3.1 \mathrm{O}$ arcabouço de verificação de modelos . . . . . . . . . . . . . . . 37

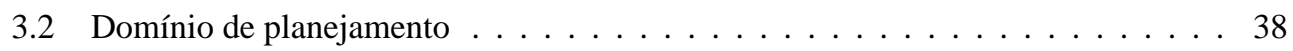

3.3 Linguagem $\mathcal{A R} \ldots \ldots \ldots \ldots \ldots$

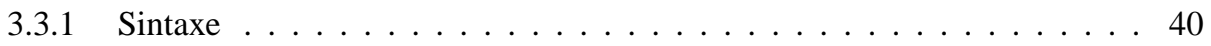

3.3.2 Semântica . . . . . . . . . . . . . . . . . . . . . 41

3.4 Especificando um domínio em $\mathcal{A R} \ldots \ldots \ldots$. . . . . . . . . 42

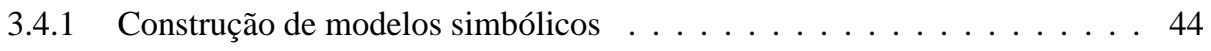

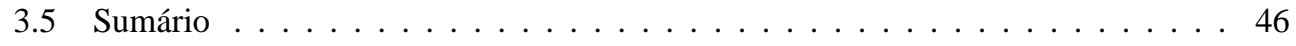

4 Planejamento para metas de alcançabilidade simples $\quad 47$

4.1 Metas de alcançabilidade simples . . . . . . . . . . . . . . . . . . . . 47

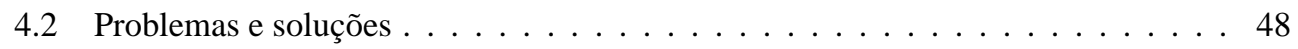

4.2.1 Caracterização das classes de soluções em CTL . . . . . . . . . . . . . 50

4.3 Algoritmos para síntese de políticas $\ldots \ldots \ldots \ldots \ldots \ldots \ldots \ldots \ldots \ldots$

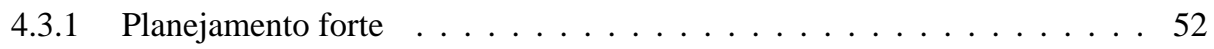

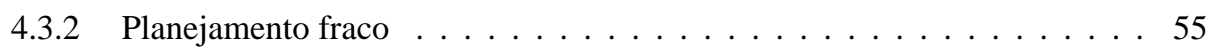

4.3 .3 Planejamento forte-cíclico $\ldots \ldots \ldots \ldots \ldots \ldots$

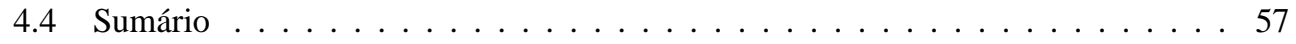

5 Planejamento para metas de alcançabilidade estendidas 59

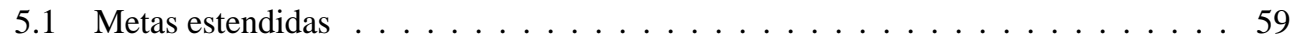

5.1.1 Metas de alcançabilidade estendidas . . . . . . . . . . . . . . . . . 61

5.1 .2 Problemas e soluções . . . . . . . . . . . . . . . . . 62

5.1.3 Subclasses de metas de alcançabilidade estendidas . . . . . . . . 63

5.2 Inadequação da lógica $\mathrm{CTL} \ldots \ldots \ldots \ldots$

5.2.1 Subclasse linear de metas de alcançabilidade estendidas . . . . . . . . . 64 
5.2.2 Subclasse ramificada de metas de alcançabilidade estendidas . . . . . . 66

5.3 A lógica temporal $\alpha$-CTL . . . . . . . . . . . . . . . . . . 68

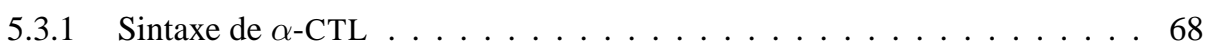

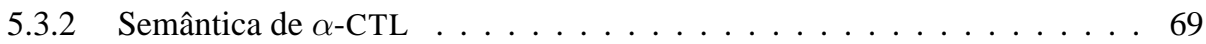

5.3.3 Um verificador de modelos para $\alpha$-CTL $\ldots \ldots \ldots \ldots \ldots$

5.4 Planejamento baseado em $\alpha$-CTL $\ldots \ldots \ldots \ldots \ldots$

5.4.1 O algoritmo de planejamento PACTL . . . . . . . . . . . . 75

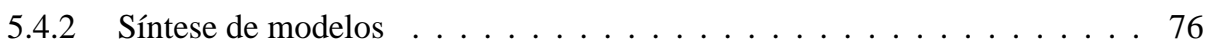

5.4.3 Propriedades formais do algoritmo PACTL . . . . . . . . . . . . 80

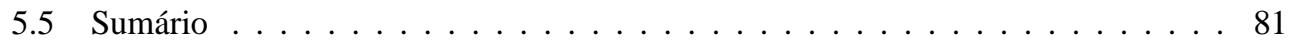

6 Qualidade das soluções em domínios quantitativos $\quad 83$

6.1 Processos de decisão markovianos . . . . . . . . . . . . . . . . 83

6.1 .1 Modelo do ambiente . . . . . . . . . . . . . . 84

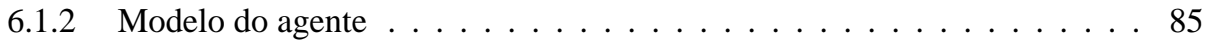

6.1 .3 Políticas ótimas $\ldots \ldots \ldots \ldots \ldots$

6.2 Planejamento quantitativo baseado em MDPs . . . . . . . . . . . . . . . . . . 89

6.2 .1 Domínios de planejamento . . . . . . . . . . . . . . . 89

6.2 .2 Problemas e soluções . . . . . . . . . . . . . . . . . 90

6.2.3 Qualidade das soluções . . . . . . . . . . . . . . . . 90

6.3 Planejamento probabilístico forte . . . . . . . . . . . . . . 95

6.3.1 Necessidade de soluções fortes . . . . . . . . . . . . . . . . . 95

6.3 .2 Domínio quantitativo $v s$ qualitativo . . . . . . . . . . . . . 96

6.3.3 Metas de alcançabilidade simples . . . . . . . . . . . . . 97

6.3.4 Metas de alcançabilidade estendidas . . . . . . . . . . . . . . 101

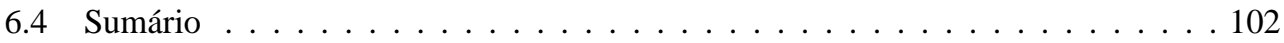

7 Conclusão $\quad 103$

7.1 Trabalhos correlatos . . . . . . . . . . . . . . . . . . 104

7.2 Contribuições desse trabalho . . . . . . . . . . . . . . . . 107 
7.3 Trabalhos futuros . . . . . . . . . . . . . . . 107

$\begin{array}{lr}\text { A Provas dos teoremas } & 109\end{array}$

A.1 Propriedades formais do algoritmo VACTL . . . . . . . . . . . . . . . . 109

A.2 Propriedades formais do algoritmo PACTL . . . . . . . . . . . . . . 111

A.3 Propriedades formais do algoritmo PPF . . . . . . . . . . . . . . 117

B Verificação e síntese de modelos usando $\alpha$-CTL 119

B.1 Especificação de modelos . . . . . . . . . . . . . . . . . . . . . . . 119

B.2 Verificador de modelos para $\alpha$-CTL . . . . . . . . . . . . . . 121

B.3 Planejador baseado em $\alpha$-CTL . . . . . . . . . . . . . . . . . . 125

$\begin{array}{lll}\text { C Teoria da decisão } & 131\end{array}$

C.1 Teoria da probabilidade . . . . . . . . . . . . . . . . 131

C.2 Teoria da utilidade . . . . . . . . . . . . . . . . 133

$\begin{array}{lr}\text { Referências Bibliográficas } & 138\end{array}$

$\begin{array}{ll}\text { Índice Remissivo } & 149\end{array}$ 


\section{Lista de Figuras}

1.1 Grafo de transições de estados para domínio do mundo dos blocos. . . . . . . . . 2

1.2 Incerteza como um jogo entre o agente e a natureza. . . . . . . . . . . . . . 5

1.3 Modelo conceitual de planejamento sob incerteza. . . . . . . . . . . . . 7

$1.4 U$ Um ambiente de planejamento sob incerteza. . . . . . . . . . . . . . . 8

1.5 Roomba: um robô aspirador de pó. . . . . . . . . . . . . . . . . . . . 10

2.1 Verificador de modelos. . . . . . . . . . . . . . . . . . . . . 15

2.2 Uma estrutura de Kripke e sua árvore de computação correspondente. . . . . . . . 17

2.3 Semântica dos operadores temporais da lógica CTL. . . . . . . . . . . . . . . 19

2.4 Sistemas distintos em CTL, mas idênticos em LTL. . . . . . . . . . . . . . . 20

2.5 Modelo de sistema com uma propriedade que não pode ser expressa em CTL. . . . 21

2.6 Funcionamento do algoritmo de ponto-fixo máximo para $\exists$ 口 $\ldots \ldots \ldots \ldots$

2.7 Funcionamento do algoritmo de ponto-fixo mínimo para $\exists \diamond p \ldots \ldots . \ldots 24$

2.8 Estrutura de Kripke sobre o conjunto $\mathbb{P}=\{p, q\} \ldots \ldots \ldots \ldots$

2.9 Árvore e diagrama de decisão binária para a fórmula $\left(p_{1} \vee p_{2}\right) \wedge\left(p_{2} \vee p_{3}\right) \ldots .29$

2.10 Compartilhamento de grafos isomorfos e eliminação de testes redundantes. . . . . 30

3.1 Planejamento baseado em verificação de modelos . . . . . . . . . . . . . 37

3.2 Domínio com assinatura $(\mathbb{P}, \mathbb{A})$, sendo $\mathbb{P}=\{p, q, r\}$ e $\mathbb{A}=\{a, b, c\} . \ldots 39$

3.3 Grafo de transições para o domínio descrito em (3.7). . . . . . . . . . . . . . . . 44

4.1 Domínio e estrutura de execução da política $\pi=\left\{\left(s_{0}, a_{01}\right),\left(s_{1}, a_{15}\right),\left(s_{2}, a_{20}\right)\right\} . \quad 49$ 
4.2 Classes de soluções em planejamento qualitativo. . . . . . . . . . . . . . . . . 51

4.3 Validação de política com relação a uma meta de planejamento. . . . . . . . . . . 51

4.4 Finalidade da função PodA. . . . . . . . . . . . . . . . . . . . . . 53

4.5 Funcionamento do algoritmo ForTe, para alcançar $s_{4}$, a partir de $s_{0}$. . . . . . . 54

4.6 Funcionamento do algoritmo FrACO, para alcançar $s_{5}$, a partir de $s_{0} . \ldots 56$

4.7 Funcionamento do algoritmo ForTECícLICO, para alcançar $s_{5}$, a partir de $s_{0}$. . . 57

5.1 Cenário para o agente "entregador". . . . . . . . . . . . . . . 59

5.2 Estrutura de execução da política estendida para o Exemplo 5.1. . . . . . . . . . . 61

5.3 Domínio de planejamento $\mathcal{D}^{1}$ e estrutura de Kripke $\mathcal{K}\left(\mathcal{D}^{1}\right)$ correspondente. . . . 64

5.4 Estrutura de execução $\mathcal{D}_{1}^{\pi_{1}}$ e estrutura de Kripke $\mathcal{K}\left(\mathcal{D}_{1}^{\pi_{1}}\right)$ correspondente. . . . . 65

5.5 Domínio de planejamento $\mathcal{D}^{2}$ e estrutura de Kripke $\mathcal{K}\left(\mathcal{D}^{2}\right)$ correspondente. . . . 66

5.6 Estrutura de execução $\mathcal{D}_{\pi_{2}}^{2}$ e estrutura de Kripke $\mathcal{K}\left(\mathcal{D}_{\pi_{2}}^{2}\right)$ correspondente. . . . . 68

5.7 Domínios e políticas como modelos temporais. . . . . . . . . . . . . . . . . 69

5.8 Planejador baseado em $\alpha$-CTL . . . . . . . . . . . . . . . . . 75

5.9 Mecanismo de síntese de modelos para operadores temporais locais. . . . . . . . . 77

5.10 Mecanismo de síntese de modelos para a fórmula $\forall \bullet \exists \odot g$. . . . . . . . . 79

6.1 Interação entre agente e ambiente dinâmico estocástico. . . . . . . . . . . . . 84

6.2 Domínio em que a solução ótima é forte-cíclica. . . . . . . . . . . . . . . . . . . 92

6.3 Domínio em que a solução ótima é estritamente fraca. . . . . . . . . . . . . . . . 93

6.4 Um domínio de planejamento probabilístico. . . . . . . . . . . . . . . . . . 98

6.5 Ordenação topológica dos estados do domínio na Figura 6.4 . . . . . . . . . . . . 98

6.6 Execução do algoritmo de planejamento probabilístico forte. . . . . . . . . . . . 999

B.1 Domínio de planejamento para meta de alcançabilidade simples $\mathcal{D}^{1}$. . . . . . . . 119

B.2 Domínio de planejamento para meta de alcançabilidade estendida $\mathcal{D}^{2}$. . . . . . 120

C.1 Comportamento irracional gerado pela violação à propriedade de transitividade. . 135

C.2 Ações com efeitos incertos vistas como loterias. . . . . . . . . . . . . . 135 


\section{Capítulo 1}

\section{Introdução}

"Failing to plan is
planning to fail."

Effie Jones

\subsection{O problema de planejamento}

$\mathrm{Na}$ vida real, todos os dias, nos deparamos com a necessidade de planejar nossas ações para que nossas metas possam ser alcançadas. De fato, a habilidade de planejar tarefas é um aspecto fundamental do comportamento inteligente e, já há mais de quarenta anos [McCarthy, 1963, Newell \& Simon, 1963], sua automatização tem sido um dos principais objetivos da pesquisa realizada na área de Inteligência Artificial [Russell \& Norvig, 2002].

\subsubsection{Planejamento clássico}

Há diversos fatores que dificultam o processo de automatização do planejamento de tarefas [Ghallab et al., 2004]. Visando simplificar esse processo, a abordagem clássica de planejamento [Korf, 1987, Weld, 1994, Fikes \& Nilsson, 1990] supõe que o ambiente de planejamento evolui de forma determinística; ou seja: (i) que não há incerteza sobre os efeitos das ações do agente; (ii) que o estado corrente do ambiente muda apenas como conseqüência das ações executadas pelo agente; e (iii) que o agente executa ações que conduzem a evolução do ambiente, de modo que um determinado estado final desejado seja alcançado. 
Com base nas suposições feitas pela abordagem clássica, a dinâmica de um ambiente de planejamento pode ser especificada, em tempo discreto, por um modelo denominado domínio de planejamento. Nesse modelo, um estado é uma configuração particular do ambiente; sendo que, em cada instante $t$, o ambiente pode estar num único estado $s_{t}$. Uma ação é um evento cuja ocorrência, controlada pelo agente, causa uma transição do instante $t$ para o instante $t+1$; fazendo com que o ambiente evolua do estado corrente $s_{t}$ para um próximo estado $s_{t+1}$.

Definição 1.1. Um domínio de planejamento clássico é definido por uma tupla $\mathcal{D}=\langle\mathcal{S}, \mathcal{A}, \mathcal{T}\rangle$, onde:

- $\mathcal{S} \neq \varnothing$ é um conjunto finito de estados possíveis do ambiente;

- $\mathcal{A} \neq \varnothing$ é um conjunto finito de ações executáveis pelo agente;

- $\mathcal{T}: \mathcal{S} \times \mathcal{A} \mapsto \mathcal{S}$ é uma função de transição de estados.

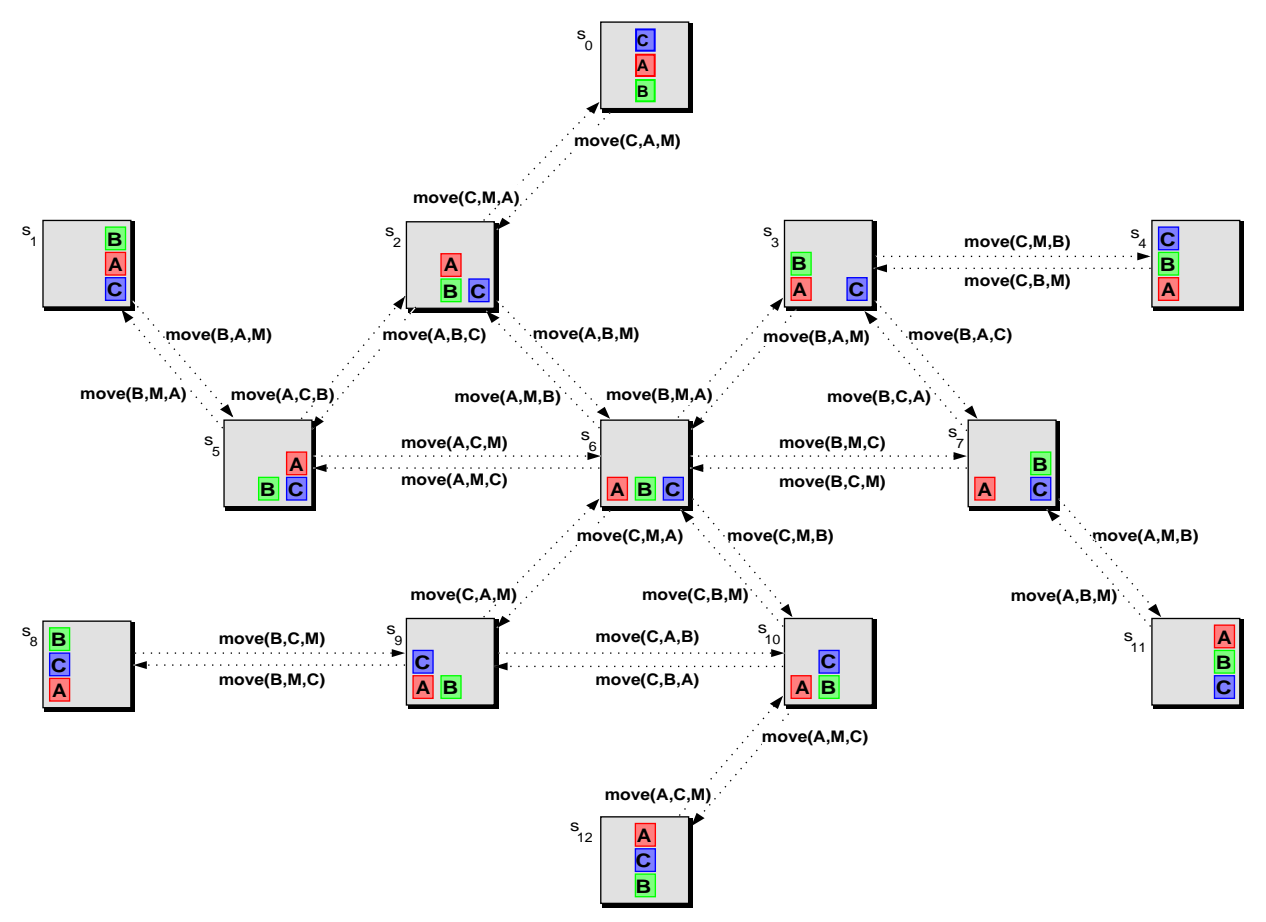

Figura 1.1: Grafo de transições de estados para domínio do mundo dos blocos.

Um domínio de planejamento pode ser descrito por meio de um grafo orientado e rotulado, denominado grafo de transições de estados ou, simplesmente, grafo de transições. Por exemplo, na Figura 1.1, podemos ver o grafo de transições para o domínio do mundo dos blocos 
[Nilsson, 1980]. Nesse grafo, os vértices representam todos os possíveis estados do mundo (i.e., configurações de três blocos A, B e C, sobre uma mesa M); enquanto os arcos, rotulados com as ações de movimentação de blocos que o agente é capaz de executar, representam todas as possíveis transições de estados no mundo.

No planejamento clássico, a meta do agente é alcançar um certo estado do ambiente, denominado estado meta, onde determinadas condições são satisfeitas. Como pode haver mais que um estado do ambiente que satisfaça tais condições, em geral, uma meta de planejamento clássico é especificada por meio de um conjunto de estados metas.

Definição 1.2. Um problema de planejamento clássico é definido por uma tupla $\mathcal{P}=\left\langle\mathcal{D}, s_{0}, \mathcal{G}\right\rangle$, onde:

- $\mathcal{D}=\langle\mathcal{S}, \mathcal{A}, \mathcal{T}\rangle$ é um domínio de planejamento clássico;

- $s_{0} \in \mathcal{S}$ é o estado inicial do ambiente;

- $\varnothing \neq \mathcal{G} \subseteq \mathcal{S}$ é um conjunto de estados metas.

Dado um problema de planejamento clássico, um planejador consiste essencialmente num algoritmo que realiza uma busca no grafo de transições de estados correspondente ao domínio de planejamento, tentando encontrar um caminho que leve o agente do estado inicial $s_{0}$ até um estado meta $s_{n} \in \mathcal{G}$. Quando tal caminho é encontrado, o planejador devolve a seqüência de ações que define esse caminho (i.e., um plano); do contrário, o planejador devolve fracasso.

Definição 1.3. Uma solução (ou plano) para um problema de planejamento clássico $\mathcal{P}=\left\langle\mathcal{D}, s_{0}, \mathcal{G}\right\rangle$ é uma seqüência de ações $\left\langle a_{0}, \ldots, a_{n-1}\right\rangle$ tal que, para $0 \leq i<n$ :

- $s_{i} \in \mathcal{S}$;

- $\mathcal{T}\left(s_{i}, a_{i}\right)=s_{i+1}$;

- $s_{n} \in \mathcal{G}$.

Exemplo 1.1 (Planejamento clássico). Considere o domínio do mundo dos blocos na Figura 1.1. Suponha que o agente esteja no estado $s_{0}$ (onde o bloco А está sobre o bloco в) e que sua meta seja alcançar um estado onde o bloco в esteja sobre o bloco A, ou seja, $\mathcal{G}=\left\{s_{1}, s_{3}, s_{4}\right\}$. Então, a seqüência de ações $\langle$ move $(\mathrm{C}, \mathrm{A}, \mathrm{M})$, move $(\mathrm{A}, \mathrm{B}, \mathrm{M})$, move $(\mathrm{B}, \mathrm{M}, \mathrm{A})\rangle$, que define um caminho que leva o agente do estado $s_{0}$ ao estado $s_{3}$, é uma solução para esse problema de planejamento. 
Embora a suposição de determinismo feita na abordagem clássica realmente simplifique a automatização da tarefa de planejamento, ainda assim, planejamento clássico está na classe de complexidade PSPACE-completo [Bylander, 1994, Bäckström, 1995]. Isso significa que o uso de representações mais compactas para os domínios pode melhorar a escalabilidade dos algoritmos de planejamento ${ }^{1}$, mas não pode reduzir a complexidade desses algoritmos.

Além disso, na prática, há várias situações em que a suposição de determinismo adotada em planejamento clássico se mostra inadequada. De fato, há pelo menos três razões para considerarmos que o ambiente de planejamento evolui de forma não-determinística (ou, equivalentemente, que as ações do agente possuem efeitos incertos): a primeira delas é que a especificação formal da dinâmica do ambiente de planejamento, geralmente incompleta, não é capaz de justificar todos os efeitos observados no mundo real (e.g., problemas de qualificação e de ramificação [Shanahan, 1997]); a segunda, é que a ocorrência de eventos exógenos ${ }^{2}$ pode interferir com as ações executadas pelo agente, modificando seus efeitos; e a terceira, é que algumas ações do agente podem realmente ter efeitos incertos (e.g., lançar uma moeda). De qualquer forma, mesmo considerando a hipótese de que o mundo real seja completamente determinístico, uma vez que desconhecemos (ou não podemos modelar) todas as causas dos efeitos observados, é razoável considerarmos que as ações do agente podem ter efeitos incertos.

\subsubsection{Planejamento sob incerteza}

Para modelar a incerteza existente acerca dos efeitos das ações do agente, podemos imaginar um jogo entre duas entidades [Blackwell \& Girshik, 1979, LaValle, 2006]:

- o agente: que planeja suas ações para atingir suas metas;

- a natureza: cujas intenções são desconhecidas e cujas ações (= eventos exógenos) podem interferir com as ações do agente, modificando seus efeitos.

No estado corrente do ambiente, denotado por $s$, o agente escolhe uma ação $a$ no conjunto $\mathcal{A}(s)$, que contém todas as ações podem ser executadas no estado $s$. Então, dependendo da ação $a$ escolhida pelo agente, a natureza escolhe uma ação $e$ no conjunto $\mathcal{E}(s, a)$, que contém todos os eventos exógenos que podem ocorrer simultaneamente à ação $a$ no estado $s$. A execução da ação $a$, simultaneamente à ocorrência do evento exógeno $e$, faz o ambiente evoluir de $s$ para um novo estado corrente $s^{\prime}$, a partir do qual o jogo prossegue analogamente.

\footnotetext{
${ }^{1}$ Permitindo lidar com domínios com mais de $10^{20}$ estados [Burch et al., 1990].

${ }^{2}$ Eventos exógenos são eventos sobre os quais o agente não tem controle.
} 


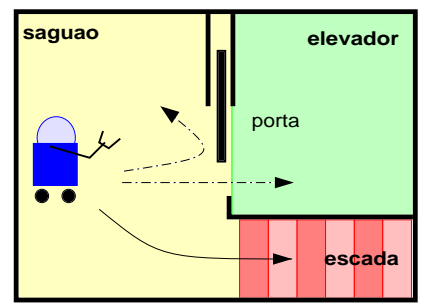

Escolhas do agente

entrar-no-elevador

$\longrightarrow$ ir-pela-escada

Figura 1.2: Incerteza como um jogo entre o agente e a natureza.

Exemplo 1.2 (Efeitos incertos). No estado s ilustrado na Figura 1.2, o agente deve escolher uma das ações no conjunto $\mathcal{A}(s)=\{$ entrar-no-elevador, ir-pela-escada $\}$. Quando ele escolhe a ação entrar-no-elevador, a natureza deve escolher uma das ações no conjunto $\mathcal{E}$ ( $s$, entrar-no-elevador) $=\{$ esperar, fechar-a-porta $\}$. Assim, mesmo executando a ação entrar-no-elevador, o agente não teria nenhuma garantia de que no instante seguinte ele estaria, de fato, dentro do elevador (pois a natureza poderia escolher fechar-a-porta, impedindo sua entrada no elevador).

Devido à interferência da natureza (Exemplo 1.2), mesmo conhecendo o estado corrente de seu ambiente, nem sempre o agente é capaz de prever, com absoluta certeza, qual será o estado resultante da execução de uma determinada ação. Sendo assim, a cada ação executada, ele precisa observar (ou sensoriar) o ambiente para determinar o seu estado corrente.

Dependendo da capacidade de percepção do agente, mesmo observando o ambiente, pode ser que ele não consiga determinar seu estado corrente com precisão (observabilidade parcial). Embora planejamento com observabilidade parcial tenha grande utilidade em aplicações práticas [Bertoli \& Pistore, 2004], trata-se de uma classe de problemas difíceis [Rintanen, 2004], para os quais existem poucos resultados satisfatórios. Por esse motivo, vamos assumir que o agente tem observabilidade completa do ambiente de planejamento, ou seja, que ele é capaz de discriminar precisamente o seu estado corrente, sem nenhuma ambigüidade.

Domínio de planejamento não-determinístico. Um domínio de planejamento não-determinístico é um modelo que expressa a "incerteza" que o agente tem acerca do comportamento da natureza. Dados um estado $s$ e uma ação $a$, esse modelo fornece ao agente uma "previsão" sobre o próximo estado do ambiente, caso a ação $a$ seja executada no estado $s$. 
Existem dois tipos básicos de domínios de planejamento não-determinístico:

- qualitativo (ou possibilístico): que supõe que o agente não tem nenhuma idéia sobre como a natureza escolhe sua ação no conjunto $\mathcal{E}(s, a)$;

- quantitativo (ou probabilístico): que supõe que o agente observou a natureza e obteve estatísticas sobre a freqüência com que ela escolhe cada uma das ações no conjunto $\mathcal{E}(s, a)$.

Em geral, um agente que dispõe de um modelo de incerteza qualitativo adota uma estratégia de seleção de ações que leva em conta apenas o fato de essas ações permitirem ou não que ele alcance sua meta.

Exemplo 1.3 (Estratégia pessimista). Considere a situação ilustrada na Figura 1.2 e suponha que a meta do agente seja alcançar o piso inferior. Para tanto, o agente pode escolher entre irpela-escada ou entrar-no-elevador. Sabendo que ao tentar entrar-no-elevador a natureza poderá fechar-a-porta, um agente que adote uma estratégia de seleção de ações pessimista escolherá ir-pela-escada. Ainda que usar o elevador seja um meio mais eficiente de alcançar sua meta, esse agente preferirá não correr riscos.

Por outro lado, um agente que dispõe de um modelo de incerteza quantitativo pode adotar uma estratégia de seleção de ações que considere também com que probabilidade cada uma das ações poderá levá-lo a alcançar sua meta e com que eficiência essa meta será alcançada; nesse caso, em geral, a estratégia adotada pelo agente é otimista com relação a essa informação.

Exemplo 1.4 (Estratégia otimista). Considere novamente a situação ilustrada na Figura 1.2, em que a meta é chegar ao piso inferior, mas suponha agora que o agente adote uma estratégia de seleção de ações otimista. Então, se o agente souber que a probabilidade da natureza escolher fechar-a-porta é muito pequena, ele correrá o risco e escolherá entrar-no-elevador.

Políticas. Quando o ambiente de planejamento evolui deterministicamente, a cada ação executada, o agente sabe exatamente qual é o próximo estado do ambiente e, portanto, pode escolher com absoluta certeza qual será a próxima ação a ser executada. Por outro lado, em ambientes não-determinísticos, o agente deve estar preparado para lidar com situações em que as coisas não acontecem conforme era esperado. 
Exemplo 1.5 (Inadequação de planos seqüenciais em ambientes não-determinísticos). Suponha que, a partir do estado corrente ilustrado na Figura 1.2, um agente otimista decida executar o plano seqüencial 〈entrar-no-elevador, sair-do-elevador〉, para alcançar sua meta de chegar ao piso inferior. Certamente, esse plano não seria de grande utilidade para o agente. Uma vez que não há garantia de que ele estará dentro do elevador após executar a ação entrar-no-elevador, também não há garantia de que ele poderá executar a ação sair-do-elevador logo em seguida.

Assim, em domínios de planejamento não-determinísticos, em vez de seqüências de ações, planos devem ser políticas. Uma política é um mapeamento de estados em ações, sintetizado a partir da especificação formal da dinâmica do ambiente (i.e., domínio de planejamento), do estado inicial desse ambiente e da meta do agente nesse ambiente (Figura 1.3). Intuitivamente, uma política define um padrão de comportamento para o agente: em cada instante, ele observa o estado corrente do ambiente e executa a ação mais apropriada para esse estado, conforme especificado pela política. Comportando-se dessa forma, o agente deve ser capaz de conduzir a evolução do ambiente, a despeito da ocorrência de eventos exógenos, de modo que sua meta possa ser alcançada.

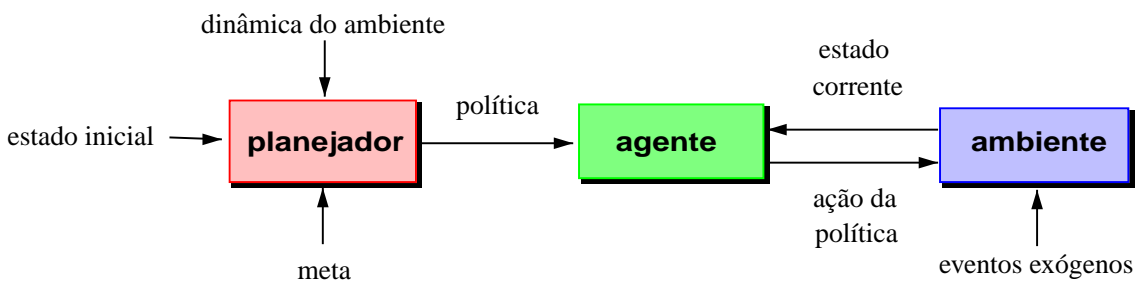

Figura 1.3: Modelo conceitual de planejamento sob incerteza.

Qualidade das soluções. Dado um problema de planejamento sob incerteza em que a meta de planejamento é especificada por meio de um conjunto de estados finais desejáveis $\mathcal{G}$, uma solução para esse problema é uma política que permite ao agente alcançar um dos estados em $\mathcal{G}$. Entretanto, como uma mesma política pode gerar diferentes caminhos de execução num ambiente não-determinístico, uma questão que surge é a seguinte: "que garantia um agente pode ter de que, seguindo uma determinada política, ele realmente alcançará sua meta?"

Na verdade, para um mesmo problema de planejamento, podem existir diferentes qualidades de políticas; cada uma delas definindo um tipo de comportamento distinto para o agente. 


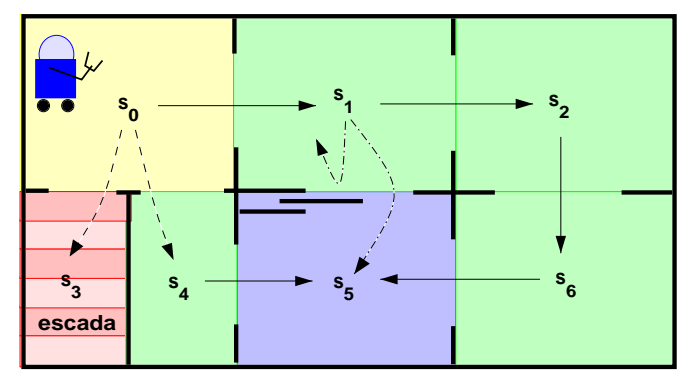

Figura 1.4: Um ambiente de planejamento sob incerteza.

O Exemplo 1.6 discute algumas qualidades de políticas e os respectivos tipos de comportamento que elas definem para um agente.

Exemplo 1.6 (Qualidade). Considere o ambiente de planejamento sob incerteza ilustrado na Figura 1.4. Nesse ambiente, quando o agente tenta mover-se de $s_{0}$ para $s_{4}$, ele pode acabar entrando em $s_{3}$ (onde há uma escada que o impede de voltar); e quando o agente tenta mover-se de $s_{1}$ para $s_{5}$, ele pode acabar permanecendo em $s_{1}$ (caso o a porta automática que separa essas duas salas se feche antes que ele consiga passar). Suponha que a meta do agente nesse ambiente, que encontra-se inicialmente no estado $s_{0}$, seja alcançar o estado $s_{5}$. Para esse problema, há três soluções distintas, com qualidades também distintas:

- $\pi_{1}=\left\{\left(s_{0}\right.\right.$, entrar-em- $\left.s_{1}\right),\left(s_{1}\right.$, entrar-em- $\left.s_{2}\right),\left(s_{2}\right.$, entrar-em- $\left.s_{6}\right),\left(s_{6}\right.$, entrar-em- $\left.\left.s_{5}\right)\right\}$

- $\pi_{2}=\left\{\left(s_{0}\right.\right.$, entrar-em- $\left.s_{1}\right),\left(s_{1}\right.$, entrar-em- $\left.\left.s_{5}\right)\right\}$

- $\pi_{3}=\left\{\left(s_{0}\right.\right.$, entrar-em- $\left.s_{4}\right),\left(s_{4}\right.$, entrar-em- $\left.\left.s_{5}\right)\right\}$

Seguindo a política $\pi_{1}$, o agente necessariamente alcança a meta de planejamento (estratégia do tipo "pessimista"); seguindo a política $\pi_{2}$, o agente alcança a meta, desde que, em algum momento, ele consiga ser rápido o suficiente para passar antes que a porta automática se feche (estratégia do tipo "tentativa e erro"); e, finalmente, seguindo a política $\pi_{3}$, o agente pode alcançar sua meta, mas não tem nenhuma garantia disso (estratégia do tipo "otimista").

\subsection{Sobre a tese}

O principal desafio da área de planejamento automatizado [Ghallab et al., 2004] é desenvolver algoritmos de planejamento eficientes para obtenção de soluções confiáveis para problemas reais. 
Esses problemas, além de possuírem enormes espaços de estados, em geral, também envolvem tomada de decisão sob incerteza, o que torna o desafio ainda maior.

Recentemente, avanços na área de planejamento automatizado têm levado a um crescente interesse em métodos automáticos para planejamento sob incerteza. Em conseqüência disso, várias abordagens interessantes foram propostas; incluindo planejamento baseado em análise meiosfins [Kushmerick et al., 1994], teoria da decisão [Boutilier et al., 1999, Thiébaux et al., 2006, Trevizan et al., 2007], verificação de modelos [Giunchiglia \& Traverso, 1999, Jensen et al., 2004, Bertoli \& Pistore, 2004], busca heurística [Brafman \& Hoffmann, 2004, Bonet \& Geffner, 2005, Bryce \& Kambhampati, 2004] e jogos adversariais [Jensen et al., 2001].

A despeito dessa grande variedade de abordagens propostas para lidar com planejamento sob incerteza, pelo menos três problemas importantes continuam em aberto na área: escalabilidade dos métodos de planejamento, expressividade das especificações de metas e qualidade das soluções obtidas pelos planejadores.

Com essa tese, contribuímos principalmente em dois desses problemas: expressividade das especificações de metas e qualidade das soluções obtidas para essas metas. Ademais, apesar de não tratarmos diretamente o problema da escalabilidade, adotamos uma abordagem de planejamento baseada em verificação de modelos [Giunchiglia \& Traverso, 1999] que, aliada ao uso de técnicas simbólicas [Burch et al., 1990], pode aumentar bastante a escalabilidade do método de planejamento que propomos.

\subsubsection{Motivação}

A Competição Internacional de Planejamento (IPC) é um evento bianual, realizado em paralelo com a Conferência Internacional de Planejamento Automatizado e Escalonamento (ICAPS), em que planejadores são avaliados, não apenas em termos de desempenho ao sintetizar uma solução, mas também com relação à qualidade dessa solução. Essa competição tem como objetivo definir o estado-da-arte em planejamento, avaliando e motivando a pesquisa existente, bem como identificando novas linhas de pesquisa para o futuro.

Com relação a metas de planejamento, a chamada oficial da última IPC $^{3}$, realizada em 2006, define o seguinte:

${ }^{3}$ http://zeus.ing.unibs.it/ipc-5 
"The competition will focus only on planning problems for goal reachability with unit costs, as they are the simplest generalization of classical planning to the nondeterministic setting, and also as the majority of existing planners fall into this category."

[Bonet \& Geffner, 2006]

De fato, a maioria dos planejadores descritos na literatura da área de planejamento automatizado trata apenas problemas de planejamento para metas de alcançabilidade simples, como aquelas do planejamento clássico (Seção 1.1.1), especificadas por meio de conjuntos de estados finais desejados. Como podemos perceber, até mesmo os planejadores que representam o estado-daarte nessa área ainda estão principalmente voltados para uma classe de metas com expressividade bastante limitada. Não obstante esse fato, planejadores capazes de lidar com especificações de metas mais expressivas (i.e., metas estendidas) têm despertado cada vez mais interesse na comunidade de planejamento ${ }^{4}$. Um exemplo de meta mais expressiva bastante interessante, que ainda não foi devidamente tratada pela comunidade de planejamento, é apresentada no Exemplo 1.7.

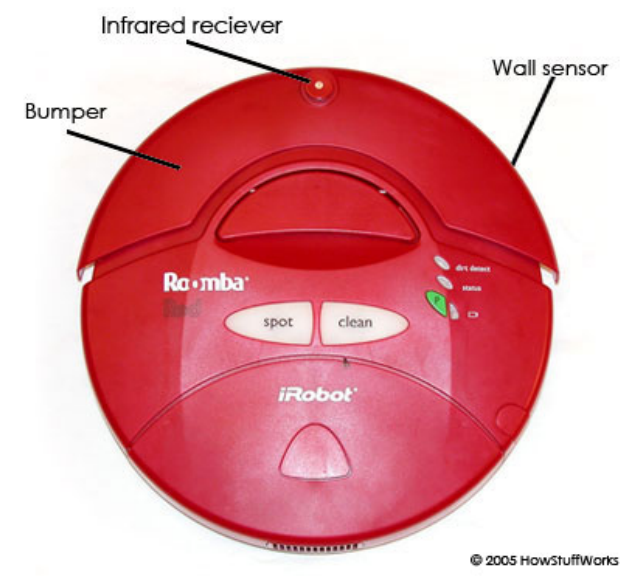

Figura 1.5: Roomba: um robô aspirador de pó.

\footnotetext{
${ }^{4}$ Veja Artificial Intelligence Journal Special Issue on Advances in Automated Plan Generation Systems - 2007, (http://users.rsise.anu.edu.au/thiebaux/AIJCFP.html).
} 
Exemplo 1.7 (Meta estendida). Roomba (Figura 1.5) é um robô bastante popular, comercializado pela empresa norte-americana iRobot ${ }^{\mathrm{TM}}$. Esse robô é um aspirador de pó capaz de se dirigir automaticamente a uma estação de recarga de bateria, sempre que ele detecta que sua bateria está fraca. Há um projeto de futuramente integrar esse aspirador a um "carpete inteligente", que seria capaz de mapear o ambiente e fornecer informações tais como conexões entre cômodos, posição do robô e localizações das estações de recarga e da sujeira. Essa integração permitirá que o robô possa planejar sua rota de limpeza de modo que sempre que sua bateria estiver fraca, ele esteja suficientemente próximo a uma estação de recarga. Dessa forma, ele poderá dirigirse a essa estação, recarregar sua bateria, e retornar ao ponto em que estava para continuar a limpeza. Note que as estações de recarga não precisarão estar na rota de limpeza planejada pelo robô, mas apenas serem facilmente acessíveis a partir dessa rota. Como podemos ver, a meta desse robô é um tipo de meta estendida, pois não há como especificá-la usando apenas um conjunto de estados finais desejados, a serem alcançados ao término da execução da política.

O problema com o uso de metas estendidas é que, quanto mais expressivas são essas metas, mais difícil se torna o processo de síntese de políticas capazes de alcançá-las (em geral, um aumento de expressividade implica num aumento de complexidade - vide Seção 5.1). Assim, uma questão que motivou o desenvolvimento dessa tese é a seguinte:

Seria possível aumentar a expressividade das metas de planejamento clássico, sem aumentar consideravelmente a complexidade dos métodos de planejamento necessários para tratá-las?"

Uma outra motivação importante está relacionada à garantia da qualidade das soluções obtidas para essas especificações de metas mais expressivas. Embora aplicações práticas de planejamento requeiram soluções com um alto nível de confiabilidade, o uso de métodos formais [Saiedian, 1996, Clarke \& Wing, 1996] para garantir a qualidade dos planos sintetizados pelos planejadores automáticos tem recebido relativamente pouca atenção. De fato, na literatura da área, a maioria dos trabalhos que empregam métodos formais para planejamento consideram apenas planejamento clássico (e.g., [Levesque et al., 1997, Reiter, 2001, Pereira \& Barros, 2002, Pereira \& Barros, 2004a, Pereira \& Barros, 2004b, Pereira \& Barros, 2004c]), havendo bem poucas referências ao uso de métodos formais para síntese ou validação de planos para ambientes de planejamento não-determinísticos. 


\subsubsection{Objetivos}

O objetivo dessa tese é propor um arcabouço de planejamento para metas de alcançabilidade estendidas (i.e., uma classe de metas que permitem impor restrições a serem preservadas ao longo de toda a execução da política sintetizada), em ambientes de planejamento não-determinísticos e completamente observáveis. Esse arcabouço, fundamentado no uso de métodos formais, permite tanto a especificação de metas com qualidades desejadas (bem mais expressivas do que aquelas consideradas em planejamento clássico), quanto a síntese e validação de políticas para essas metas (usando algoritmos implementados com base na verificação formal do domínio de planejamento, com relação à especificação de meta). Além disso, mostramos como o arcabouço proposto pode ser usado quando as probabilidades das transições no modelo do domínio de planejamento são conhecidas.

\subsubsection{Contribuições}

As principais contribuições dessa tese são as seguintes:

- definição de uma classe de metas mais expressivas que aquelas do planejamento clássico (denominadas metas de alcançabilidade estendidas) e que permitem especificar a qualidade da solução desejada (conforme ressaltamos na Subseção 1.2.1, essa classe de metas ainda não havia sido devidamente tratada pela comunidade de planejamento);

- definição de uma lógica temporal cuja semântica permite o tratamento da classe de metas de alcançabilidade estendidas (conforme mostramos na Seção 5.2, a lógica CTL, que pode ser usada para especificar metas de alcançabilidade simples, não é adequada para o tratamento de metas de alcançabilidade estendidas);

- formulação original de um arcabouço para resolver problemas de planejamento em domínios não-determinísticos e completamente observáveis, envolvendo metas de alcançabilidade estendidas com qualidade de solução desejada (até onde sabemos, esse é o primeiro trabalho que propõe métodos eficientes e escaláveis para tratamento de metas de alcançabilidade estendidas, que inclui metas de alcançabilidade simples do planejamento clássico como um caso especial);

- garantir soluções de qualidade através do uso de algoritmos corretos e completos, baseados em métodos formais. 


\subsection{Organização}

O restante dessa tese está organizado conforme segue. No Capítulo 2, introduzimos o arcabouço de verificação de modelos e apresentamos os algoritmos para verificação de propriedades especificadas em CTL. No Capítulo 3, mostramos como o arcabouço de verificação de modelos pode ser adaptado para resolver problemas de planejamento em ambientes não-determinísticos e completamente observáveis. No Capítulo 4, caracterizamos as diferentes classes de soluções para metas de alcançabilidade simples, com base na semântica de CTL, e apresentamos os algoritmos capazes de obter soluções em cada uma dessas classes. No Capítulo 5, definimos a classe de metas de alcançabilidade estendidas e mostramos porquê a semântica de CTL é inadequada para tratar essa classe de metas. Em seguida, definimos a lógica $\alpha$-CTL e, com base em sua semântica, mostramos como planejamento para metas de alcançabilidade estendidas (com requisitos de qualidade específicos) pode ser implementado dentro do arcabouço de verificação de modelos. No Capítulo 6, discutimos como podemos garantir a qualidade das soluções quando dispomos de um modelo de ambiente de planejamento em que as probabilidades das transições causadas pelas ações são conhecidas e apresentamos um algoritmo para planejamento probabilístico forte. Finalmente, no Capítulo 7, discutimos trabalhos correlatos, apresentamos as principais contribuições desse trabalho e apontamos alguns possíveis trabalhos futuros. 


\section{Capítulo 2}

\section{Verificação de modelos}

\subsection{O problema de verificação}

Dados um modelo $\mathcal{K}$, descrevendo a dinâmica de um sistema, e uma fórmula $\varphi$, descrevendo uma propriedade, verificação de modelos [Müller-Olm et al., 1999] consiste em resolver o problema $\mathcal{K} \stackrel{?}{\vDash} \varphi$. Nesse contexto, um verificador de modelos (Figura 2.1) é um algoritmo que recebe como entrada um par $(\mathcal{K}, \varphi)$ e visita sistematicamente os estados do modelo $\mathcal{K}$, verificando a validade da propriedade $\varphi$. Quando todos os estados do modelo $\mathcal{K}$ satisfazem a propriedade $\varphi$, o verificador devolve sucesso; caso contrário, o verificador devolve um contra-exemplo (e.g., um estado do modelo $\mathcal{K}$ onde a propriedade $\varphi$ é violada).

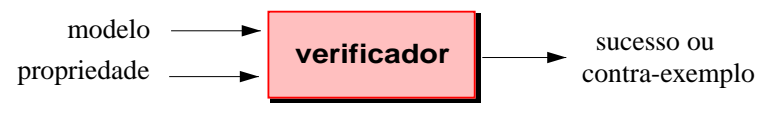

Figura 2.1: Verificador de modelos.

\subsection{Modelo computacional}

Nessa seção, definimos o modelo computacional de verificação de modelos; mais precisamente, definimos a estrutura utilizada para especificar a dinâmica do sistema, bem como a linguagem utilizada para especificar as propriedades a serem verificadas nesse sistema. 


\subsubsection{Estruturas de Kripke}

Seja $\mathbb{P} \neq \varnothing$ um conjunto finito de proposições atômicas. Formalmente, o modelo do sistema a ser verificado é uma estrutura de Kripke sobre $\mathbb{P}$.

Definição 2.1. Uma estrutura de Kripke sobre $\mathbb{P}$ é uma tupla $\mathcal{K}=\langle\mathcal{S}, \mathcal{T}, \mathcal{L}\rangle$, onde:

- $\mathcal{S} \neq \varnothing$ é um conjunto finito de estados;

- $\mathcal{T}: \mathcal{S} \mapsto 2^{\mathcal{S}}$ é uma função de transição de estados;

- $\mathcal{L}: \mathcal{S} \mapsto 2^{\mathbb{P}}$ é uma função de interpretação de estados.

Intuitivamente, as proposições em $\mathbb{P}$ representam propriedades dos estados do sistema e $\mathcal{L}(s)$ denota o conjunto de proposições atômicas que descrevem um estado particular $s \in \mathcal{S}$. Ademais, para todo estado $s \in \mathcal{S}$, assumimos que o conjunto $\mathcal{L}(s)$ é consistente e que $\mathrm{T} \in \mathcal{L}(s)$.

Originalmente, estruturas de Kripke foram propostas como um modelo semântico para lógicas modais [Kripke, 1963], cujas fórmulas utilizam a modalidade $\square$ para expressar necessidade (e a modalidade dual $\diamond$, para expressar possibilidade). Nesse contexto, os estados da estrutura de Kripke correspondem a "mundos possíveis", em que diferentes fatos (proposições) podem ser observados (satisfeitas); enquanto as transições correspondem a acessos que permitem transitar entre esses mundos. Assim, dizer que um fato é necessário (ou possível) num mundo $s \in \mathcal{S}$ significa que tal fato é observado em todo (ou algum) mundo acessível a partir de $s$.

Exemplo 2.1 (Modelo modal). Interpretando a estrutura de Kripke da Figura 2.2-a como um modelo modal, temos que o estado $s_{0}$ satisfaz a fórmula $\diamond q$, pois q é observado em $s_{1}$, acessivel a partir de $s_{0}$; porém, esse mesmo estado $\left(s_{0}\right)$ não satisfaz a fórmula $\square$ q, pois q não é observado em $s_{4}$, que também é acessivel a partir de $s_{0}$.

Por outro lado, no contexto de verificação de modelos, os estados numa estrutura de Kripke denotam "instantes" no tempo e, portanto, as modalidades $\square$ e $\diamond$ são usadas para expressar aspectos temporais. Em outras palavras, uma estrutura de Kripke é interpretada como um modelo temporal [Emerson, 1990].

Quando um estado particular numa estrutura de Kripke $\mathcal{K}$ é designado como estado inicial, essa estrutura pode ser desdobrada numa árvore infinita, enraizada nesse estado. Caso a função de transição em $\mathcal{K}$ seja parcial (i.e., não esteja definida para todos os estados em $\mathcal{S}$ ), adicionamos a cada estado terminal em $\mathcal{K}$ uma transição reflexiva. A justificativa para essas transições reflexivas é que, sendo a árvore obtida um modelo temporal para a estrutura de Kripke desdobrada, estados 


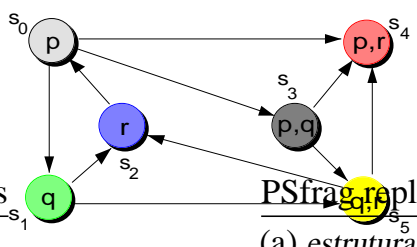

(a) estrutura de Kripke

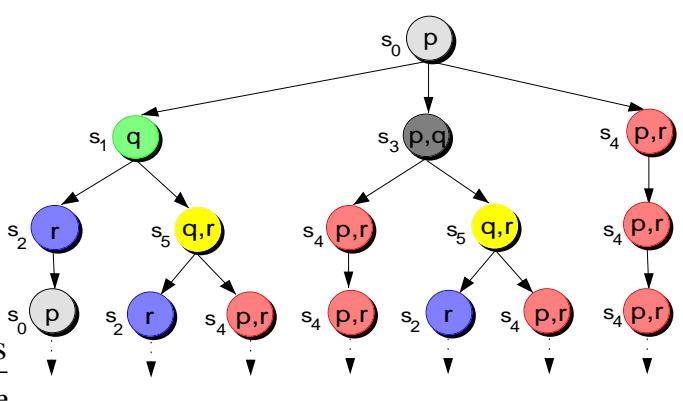

(b) árvore de computação

Figura 2.2: Uma estrutura de Kripke e sua árvore de computação correspondente.

terminais nessa estrutura devem persistir infinitamente no tempo. A árvore resultante do desdobramento de uma estrutura de Kripke representa todos os caminhos possíveis de computação do sistema modelado e, por esse motivo, tal árvore é denominada árvore de computação.

Exemplo 2.2 (Modelo temporal). Na estrutura de Kripke apresentada na Figura 2.2-a, o estado $s_{4}$ é terminal; ou seja, não existe transição desse estado para nenhum outro. Portanto, quando essa estrutura de Kripke é interpretada como um modelo temporal (árvore de computação apresentada na Figura 2.2-b), o estado $s_{4}$ deve persistir infinitamente no tempo.

Uma árvore de computação, obtida pelo desdobramento de uma estrutura de Kripke $\mathcal{K}$, a partir de um estado $s$, é denotada por $\Upsilon_{\mathcal{K}}^{s}$. Também usamos $\Upsilon_{\mathcal{K}}^{s}$ para denotar o conjunto de todos os caminhos que partem do estado $s$ na estrutura de Kripke $\mathcal{K}$. Um caminho numa árvore de computação $\Upsilon_{\mathcal{K}}^{s}$ é uma seqüência infinita de estados $\rho=\left\langle s=\rho_{0}, \rho_{1}, \ldots\right\rangle \in \mathcal{S}^{\omega}$, onde $\mathcal{S}^{\omega}$ denota o conjunto de todas as sequiências infinitas sobre $\mathcal{S}$, tal que $\rho_{i} \in \mathcal{T}\left(\rho_{i-1}\right)$, para $i>0$.

\subsubsection{Lógicas temporais}

Em geral, para especificar propriedades a serem verificadas num sistema, utilizamos lógicas temporais (i.e., lógicas modais cujas modalidades expressam aspectos temporais [Emerson, 1990]). Nessas lógicas, os estados na árvore de computação (modelo temporal do sistema) representam instantes no tempo e o valor de uma proposição depende do instante de tempo considerado. Há dois tipos básicos de lógicas temporais: aquelas de tempo linear e aquelas de tempo ramificado. Essencialmente, o que as distingue é o modo como elas lidam com as ramificações na árvore de computação: enquanto as lógicas de tempo linear consideram que cada instante no tempo 
tem um único futuro possível (semântica baseada em caminhos), as lógicas de tempo ramificado consideram que, para cada instante no tempo, pode haver vários futuros possíveis (semântica baseada em estados).

A lógica de tempo linear. LTL (Linear Time Logic) [Pnueli, 1977] é uma lógica temporal de tempo linear, cujas fórmulas permitem especificar propriedades sobre caminhos numa árvore de computação. As fórmulas LTL são construídas a partir dos seguintes operadores temporais:

- $\bigcirc$ (no próximo estado)

- $\square$ (invariantemente, em todos os estados futuros)

- $\diamond$ (finalmente, em algum estado futuro)

- $\sqcup$ (até em algum estado futuro)

As fórmulas LTL são avaliadas com relação a um caminho particular $\rho$ numa árvore de computação $\Upsilon_{\mathcal{K}}^{s}$ : a fórmula $O \varphi$ requer que $\varphi$ seja satisfeita no estado $\rho_{1}$; a fórmula $\square \varphi$ requer que $\varphi$ seja satisfeita em todos os estados de $\rho$; a fórmula $\diamond \varphi$ requer que $\varphi$ seja satisfeita em algum estado de $\rho$ e; finalmente, a fórmula $\varphi_{1} \sqcup \varphi_{2}$ requer que $\varphi_{2}$ seja satisfeita em algum estado $\rho_{j}$ e que $\varphi_{1}$ seja satisfeita em todo estado $\rho_{i}$, tal que $i<j$. A modalidade $\square$ funciona como um quantificador universal sobre estados de um caminho, enquanto a modalidade $\diamond$ funciona como uma espécie de quantificador existencial.

Definição 2.2. O conjunto de fórmulas LTL é definido indutivamente como:

$\varphi \doteq p \in \mathbb{P}\left|\neg \varphi_{1}\right| \varphi_{1} \wedge \varphi_{2}\left|\varphi_{1} \vee \varphi_{2}\right| \bigcirc \varphi_{1}\left|\square \varphi_{1}\right| \varphi_{1} \sqcup \varphi_{2}$

O operador temporal $\diamond$ é definido como:

- $\diamond \varphi \doteq T \sqcup \varphi$

Definição 2.3. Sejam $\mathcal{K}$ uma estrutura de Kripke, s um estado dessa estrutura e $\varphi$ uma fórmula LTL. A semântica das fórmulas LTL é definida como segue:

- $s \vDash \varphi$ se e só se $\rho \vDash \varphi$, para todo $\rho \in \Upsilon_{\mathcal{K}}^{s}$;

- $\rho \vDash p$ se e só se $p \in \mathcal{L}\left(\rho_{0}\right)$;

- $\rho \vDash \neg \varphi_{1}$ se e só se $\rho \not \varphi_{1}$;

- $\rho \vDash \varphi_{1} \wedge \varphi_{2}$ se e só se $\rho \vDash \varphi_{1}$ e $\rho \vDash \varphi_{2}$;

- $\rho \vDash \varphi_{1} \vee \varphi_{2}$ se e só se $\rho \vDash \varphi_{1}$ ou $\rho \vDash \varphi_{2}$;

- $\rho \vDash O \varphi_{1}$ se e só se $\rho_{1} \vDash \varphi_{1}$;

- $\rho \vDash \square \varphi_{1}$ se e só se, para $i \geq 0, \rho_{i} \vDash \varphi_{1}$;

- $\rho \vDash \varphi_{1} \sqcup \varphi_{2}$ se e só se existe $j \geq 0$ tal que $\rho_{j} \vDash \varphi_{2}$ e, para todo $i<j, \rho_{i} \vDash \varphi_{1}$. 
A lógica de tempo ramificado. CTL (Computation Tree Logic) [Clarke \& Emerson, 1982] é uma lógica temporal de tempo ramificado, cujas fórmulas permitem especificar propriedades quantificadas sobre caminhos numa árvore de computação. Na lógica CTL, operadores temporais (Figura 2.3) devem ser imediatamente precedidos por um quantificador de caminhos ( $\exists$ ou $\forall$ ):

- $\exists \circ$ (em algum próximo estado)

- $\exists \square$ (invariantemente, em algum dos estados futuros)

- $\exists \diamond$ (finalmente, em algum dos estados futuros)

- $\exists \sqcup$ (até, em algum estado futuro)

- $\forall \mathrm{O}$ (em todo próximo estado)

- $\forall \square$ (invariantemente, em todos os estados futuros)

- $\forall \diamond$ (finalmente, em todos os estados futuros)

- $\forall \sqcup$ (até, em todos os estados futuros)

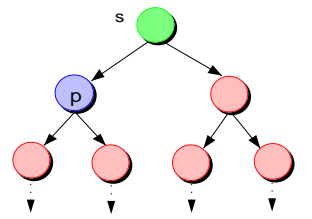

(a) $s \vDash \exists \bigcirc p$

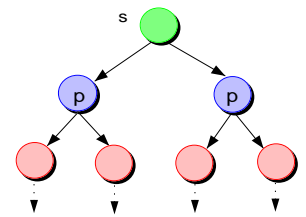

(e) $s \vDash \forall \bigcirc p$

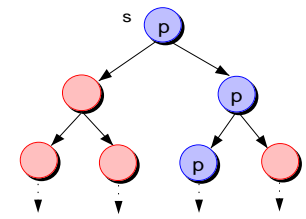

(b) $s \vDash \exists \square p$

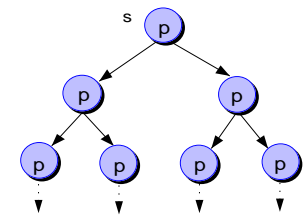

(f) $s \vDash \forall \square p$

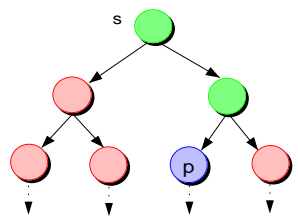

(c) $s \vDash \exists \diamond p$

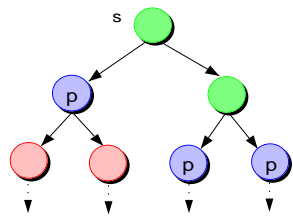

(g) $s \vDash \forall \diamond p$

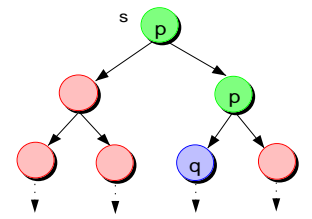

(d) $s \vDash \exists(p \sqcup q)$

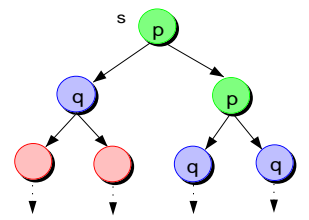

(h) $s \vDash \forall(p \sqcup q)$

Figura 2.3: Semântica dos operadores temporais da lógica CTL.

Definição 2.4. O conjunto de fórmulas CTL é definido indutivamente como:

$\varphi \doteq p \in \mathbb{P}\left|\neg \varphi_{1}\right| \varphi_{1} \wedge \varphi_{2}\left|\varphi_{1} \vee \varphi_{2}\right| \exists \bigcirc \varphi_{1}\left|\forall \bigcirc \varphi_{1}\right| \exists \square \varphi_{1}\left|\forall \square \varphi_{1}\right| \exists\left(\varphi_{1} \sqcup \varphi_{2}\right) \mid \forall\left(\varphi_{1} \sqcup \varphi_{2}\right) \bullet$

Os operadores temporais $\exists \diamond \mathrm{e} \forall \diamond$ são definidos conforme a seguir:

- $\exists \diamond \varphi_{2} \doteq \exists\left(T \sqcup \varphi_{2}\right)$

- $\forall \diamond \varphi_{2} \doteq \forall\left(T \sqcup \varphi_{2}\right)$ 
Definição 2.5. Sejam $\mathcal{K}$ uma estrutura de Kripke, s um estado dessa estrutura e $\varphi$ uma fórmula CTL. A semântica das fórmulas CTL é definida como segue:

- $s \vDash p$ se e só se $p \in \mathcal{L}(s)$;

- $s \vDash \neg \varphi_{1}$ se e só se $s \not \varphi_{1}$;

- $s \vDash \varphi_{1} \wedge \varphi_{2}$ se e só se $s \vDash \varphi_{1}$ e $s \vDash \varphi_{2}$;

- $s \vDash \varphi_{1} \vee \varphi_{2}$ se e só se $s \vDash \varphi_{1}$ ou $s \vDash \varphi_{2}$;

- $s \vDash \exists \bigcirc \varphi_{1}$ se e só se, para algum caminho $\rho \in \Upsilon_{\mathcal{K}}^{s}, \rho_{1} \vDash \varphi_{1}$;

- $s \vDash \forall \bigcirc \varphi_{1}$ se e só se, para todo caminho $\rho \in \Upsilon_{\mathcal{K}}^{s}, \rho_{1} \vDash \varphi_{1}$;

- $s \vDash \exists \square \varphi_{1}$ se e só se, para algum caminho $\rho \in \Upsilon_{\mathcal{K}}^{s}$ e todo $i \geq 1, \rho_{i} \vDash \varphi_{1}$;

- $s \vDash \forall \square \varphi_{1}$ se e só se, para todo caminho $\rho \in \Upsilon_{\mathcal{K}}^{s}$ e todo $i \geq 1, \rho_{i} \vDash \varphi_{1}$;

- $s \vDash \exists\left(\varphi_{1} \sqcup \varphi_{2}\right)$ se e só se para algum caminho $\rho \in \Upsilon_{\mathcal{K}}^{s}$, existe $j \geq 0$ tal que $\rho_{j} \vDash \varphi_{2}$ e, para todo $i<j, \rho_{i} \vDash \varphi_{1}$;

- $s \vDash \forall\left(\varphi_{1} \sqcup \varphi_{2}\right)$ se e só se para todo caminho $\rho \in \Upsilon_{\mathcal{K}}^{s}$, existe $j \geq 0$ tal que $\rho_{j} \vDash \varphi_{2}$ e, para todo $i<j, \rho_{i} \vDash \varphi_{1}$.

Comparações entre LTL e CTL. Geralmente, lógicas são comparadas sob dois aspectos principais: expressividade e complexidade.

Em termos de expressividade, as lógicas LTL e CTL são incomparáveis ${ }^{1}$ [Vardi, 1998b, Emerson \& Halpern, 1986]. Devido à forma como as semânticas dessas lógicas são definidas, CTL é capaz de distinguir situações que são consideradas idênticas em LTL (Exemplo 2.3). Ademais, devido à ausência de quantificadores em LTL, não há como especificar propriedades existenciais nessa lógica. Porém, isso não significa que LTL seja menos expressiva que CTL. Também há propriedades que podem ser especificadas em LTL, mas não em CTL (Exemplo 2.4).
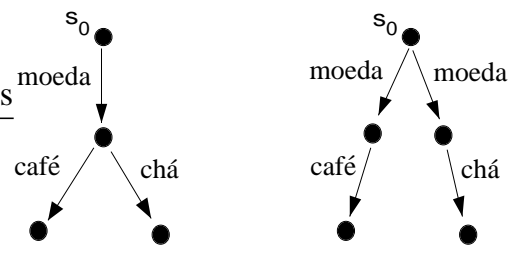

Figura 2.4: Sistemas distintos em CTL, mas idênticos em LTL.

\footnotetext{
${ }^{1}$ Essas lógicas são fragmentos de uma lógica mais expressiva (CTL ${ }^{\star}$ [Emerson \& Halpern, 1986]), que combina tempo linear e ramificado num mesmo formalismo.
} 
Exemplo 2.3 (Sistemas que não podem ser distinguidos pela semântica de LTL). Considere os sistemas de venda automática apresentados na Figura 2.4: no primeiro, a inserção de uma moeda causa uma transição para um estado onde pode-se escolher entre café ou chá; no segundo, a inserção de uma moeda causa uma transição não-determinística para um estado a partir do qual não há escolha. Embora esses sistemas sejam realmente distintos, a semântica baseada em caminhos da lógica LTL não é capaz de distingui-los; já que todos os caminhos que iniciam em $s_{0}$ são iguais, em ambos os sistemas.

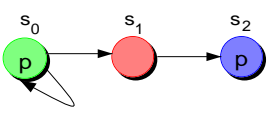

(a) estrutura de Kripke

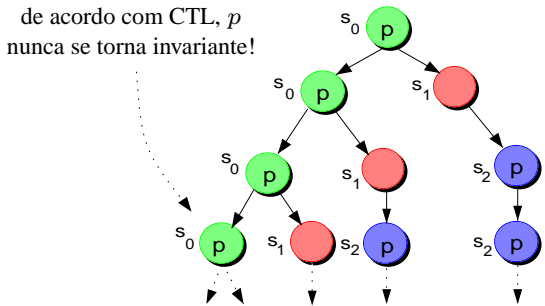

(b) árvore de computação

Figura 2.5: Modelo de sistema com uma propriedade que não pode ser expressa em CTL.

Exemplo 2.4 (Propriedade que não pode ser expressa em CTL). A propriedade "em todo caminho, finalmente p torna-se invariante" pode ser escrita em LTL como $\diamond \square p$. Em CTL, devido à restrição imposta por sua sintaxe, essa fórmula teria que ser escrita como $\forall \diamond \forall \square p$, que não expressa a propriedade desejada. De fato, essa fórmula CTL especifica que "em todo caminho, finalmente, há um estado a partir do qual, em todos os caminhos, a propriedade p é invariante". Na Figura 2.5, temos o modelo de um sistema que apresenta (e satisfaz) a propriedade especificada pela fórmula LTL, mas que não pode satisfazer a fórmula CTL: quando os caminhos são avaliados independentemente (LTL), em todos eles há um ponto onde finalmente p torna-se invariante; por outro lado, quando os caminhos são avaliados simultaneamente (CTL), como o estado $s_{0}$ sempre tem um estado sucessor onde p é falso $\left(s_{1}\right)$, não existe um ponto a partir do qual p torna-se invariante em todos os caminhos.

Em termos de complexidade, em geral, os algoritmos de verificação para CTL são muito mais eficientes que aqueles para LTL. Considere um modelo de tamanho $m$ (número de vértices e transições) e uma fórmula temporal de tamanho $n$ (número de sub-fórmulas): enquanto a complexidade de tempo dos algoritmos de verificação para CTL é $\mathcal{O}(m \times n)$, a complexidade de 
tempo dos algoritmos para LTL é $\mathcal{O}\left(m \times 2^{n}\right)$ [Schnoebelen, 2003]. Ademais, como os algoritmos para LTL são PSPACE-completos, é improvável que esse limite superior de tempo possa ser reduzido [Vardi, 1998a, Schnoebelen, 2003]. Note que essa comparação tradicionalmente feita entre LTL e CTL considera apenas a complexidade de pior caso; porém, como essas lógicas têm expressividades incomparáveis, uma comparação mais significativa deveria levar em conta apenas o fragmento comum ${ }^{2}$ dessas lógicas, ou seja, o conjunto de propriedades que podem ser especificadas tanto em LTL quanto em CTL.

\subsection{Verificação baseada em CTL}

Nessa subseção, apresentamos um algoritmo que efetivamente percorre o modelo do sistema, representado por uma estrutura de Kripke, verificando a validade de uma determinada propriedade, especificada por uma fórmula da lógica CTL. Esse algoritmo é implementado com base numa caracterização de ponto-fixo para os operadores temporais da lógica CTL.

\subsubsection{Caracterização de ponto-fixo}

A caracterização de ponto-fixo para os operadores temporais de CTL [Clarke \& Emerson, 1982] possibilita a criação de um algoritmo efetivo para o problema de verificação de modelos, que associa a cada fórmula $\varphi$ o conjunto de estados de $\mathcal{K}$ que a satisfazem.

Sejam $\mathcal{K}=\langle\mathcal{S}, \mathcal{T}, \mathcal{L}\rangle$ uma estrutura de Kripke e $2^{\mathcal{S}}$ o conjunto potência de $\mathcal{S}$. Como sabemos, $2^{\mathcal{S}}$ forma um reticulado sob união e intersecção, ordenado por inclusão de conjuntos, onde $A \subseteq$ $B$ se e só se $A \cup B=B$. Seja $\Gamma[Y]$ uma funcional com um símbolo $Y$ não-interpretado. Substituindo $Y$ por um elemento de $2^{\mathcal{S}}$, podemos obter uma função $\Gamma: 2^{\mathcal{S}} \mapsto 2^{\mathcal{S}}$. Por definição:

- $\Gamma$ é monotônica quando $A \subseteq B$ implica $\Gamma(A) \subseteq \Gamma(B)$.

- $\Gamma$ é $\cup$-contínua quando $A_{1} \subseteq A_{2} \subseteq \ldots$ implica $\Gamma\left(\cup_{i} A_{i}\right)=\cup_{i} \Gamma\left(A_{i}\right)$.

- $\Gamma$ é n-contínua quando $A_{1} \supseteq A_{2} \supseteq \ldots$ implica $\Gamma\left(\cap_{i} A_{i}\right)=\cap_{i} \Gamma\left(A_{i}\right)$.

Quando o conjunto $\mathcal{S}$ é finito, toda seqüência crescente de conjuntos tem um elemento máximo e toda seqüência decrescente de conjuntos tem um elemento mínimo. Assim, no caso finito, monotonicidade implica $\cup$-continuidade e $\cap$-continuidade.

\footnotetext{
${ }^{2}$ Para esse fragmento comum, algumas fórmulas em LTL podem ser exponencialmente mais curtas que aquelas correspondentes em CTL e, portanto, a verificação para LTL pode se tornar mais eficiente que para CTL. Como exemplo desse tipo de fórmula, podemos citar aquelas que especificam a existência de caminhos hamiltonianos.
} 
Um ponto-fixo de $\Gamma$ é qualquer $A$ tal que $\Gamma(A)=A$. Conforme [Tarski, 1955], um funcional monotônico, com relação à ordem de inclusão, sempre tem pontos-fixos mínimo e máximo.

Teorema 2.1 (Tarski-Knaster). Se $\Gamma[Y]$ é um funcional monotônico, então $\Gamma[Y]$ tem um pontofixo mínimo, denotado por $\mu Y . \Gamma[Y]$, e um ponto-fixo máximo, denotado por $\nu Y . \Gamma[Y]$. Se $\Gamma[Y]$ é monotônico e U-contínuo, então $\mu Y . \Gamma[Y]=\cup_{i \geq 0} \Gamma^{i}(\perp)$. Se $\Gamma[Y]$ é monotônico e n-contínuo, então $\nu Y . \Gamma[Y]=\cap_{i \geq 0} \Gamma^{i}(T)$.

Teorema 2.2 (Clarke-Emerson). Se $\mathcal{S}$ é finito, os operadores temporais globais de CTL são caracterizados por:

- $\exists \square \varphi=\nu Y \cdot(\varphi \wedge \exists \circ Y)$

- $\forall \square \varphi=\nu Y \cdot(\varphi \wedge \forall \circ Y)$

- $\exists \diamond \varphi=\mu Y .(\varphi \vee \exists \circ Y)$

- $\forall \diamond \varphi=\mu Y \cdot(\varphi \vee \forall \circ Y)$

- $\exists\left(\varphi_{1} \sqcup \varphi_{2}\right)=\mu Y \cdot\left(\varphi_{2} \vee\left(\varphi_{1} \wedge \exists \circ Y\right)\right)$

- $\forall\left(\varphi_{1} \sqcup \varphi_{2}\right)=\mu Y .\left(\varphi_{2} \vee\left(\varphi_{1} \wedge \forall \circ Y\right)\right)$

Como os operadores $\exists \circ \mathrm{e} \forall \bigcirc$ definem funções monotônicas sobre o reticulado $2^{\mathcal{S}}$, de acordo com o Teorema 2.1, existem soluções para as equações enunciadas no Teorema 2.2.

\subsubsection{Computação de pontos-fixos}

Existe uma correspondência direta entre fórmulas de CTL e conjuntos de estados que as satisfazem num determinado modelo temporal. Em particular, a fórmula $\perp$ corresponde ao conjunto $\varnothing$, enquanto a fórmula $\mathrm{T}$ corresponde ao conjunto $\mathcal{S}$. O algoritmo para computar um ponto-fixo mínimo (ou máximo) para um funcional $\Gamma[Y]$, caracterizando um operador CTL, consiste em iniciar com $\perp$ (ou T) e iterar esse funcional até que um ponto-fixo seja atingido. Para $\mathcal{S}$ finito, esse algoritmo termina em no máximo $|\mathcal{S}|+1$ iterações.

Exemplo 2.5 (Ponto-fixo máximo). Podemos usar o funcional $\Gamma[Y]=p \wedge \exists \bigcirc Y$ para computar o conjunto de estados da estrutura de Kripke na Figura 2.6-a que satisfazem a fórmula $\exists \square p$. Na primeira iteração, temos $\Gamma^{1}[\mathrm{~T}]=p \wedge \exists \bigcirc \mathrm{O}=\left\{s_{0}, s_{1}, s_{2}\right\}$; na segunda iteração, temos $\Gamma^{2}[\mathrm{~T}]=p \wedge \exists \circ(p \wedge \exists \bigcirc \mathrm{\top})=\left\{s_{0}, s_{1}\right\}$; e, finalmente, na terceira iteração, temos $\Gamma^{3}[\mathrm{~T}]=$ $p \wedge \exists \circ(p \wedge \exists \circ(p \wedge \exists \circ \mathrm{T}))=\left\{s_{0}\right\}$ (que já é o ponto-fixo máximo; pois $\Gamma^{4}[\mathrm{~T}]$ produz o mesmo resultado que $\left.\Gamma^{3}[\mathrm{\top}]\right)$. 


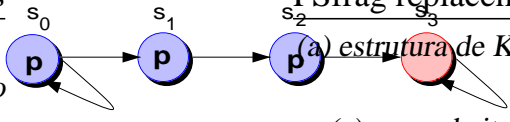

(a) estrutura de Kripke

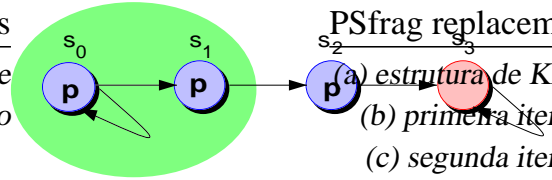

(c) segunda iteração

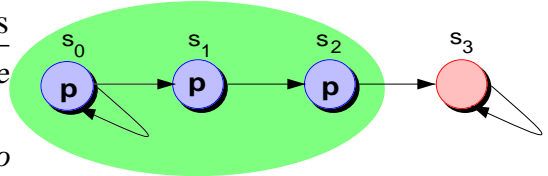

(b) primeira iteração

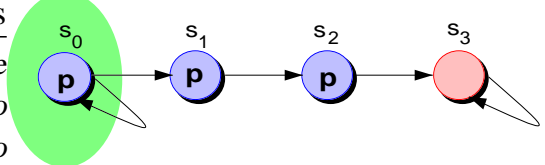

(d) terceira iteração

Figura 2.6: Funcionamento do algoritmo de ponto-fixo máximo para $\exists \square p$.

Exemplo 2.6 (Ponto-fixo mínimo). Para computar o conjunto de estados da estrutura de Kripke na Figura 2.7-a que satisfazem a fórmula $\exists \diamond p$, podemos usar o funcional $\Gamma[Y]=p \vee \exists \circ Y$. Na primeira iteração, temos $\Gamma^{1}[\perp]=p \vee \exists \bigcirc \perp=\left\{s_{1}\right\}$; na segunda iteração, temos $\Gamma^{2}[\perp]=$ $p \vee \exists \circ(p \vee \exists \circ \perp)=\left\{s_{0}, s_{1}\right\} ;$ e, finalmente, na terceira iteração, temos $\Gamma^{3}[\perp]=p \vee \exists \circ(p \vee \exists \circ$ $(p \vee \exists \bigcirc \perp))=\left\{s_{0}, s_{1}, s_{2}\right\}$ (que já é o ponto-fixo mínimo; pois $\Gamma^{4}[\perp]=\Gamma^{3}[\perp]$ ).

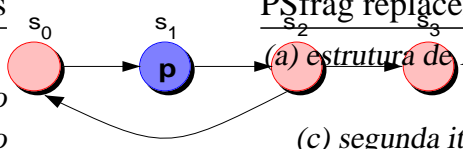

(a) estrutura de Kripke

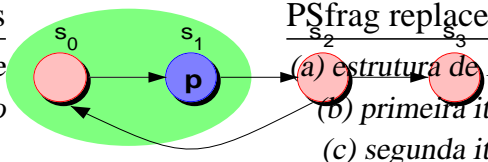

(c) segunda iteração

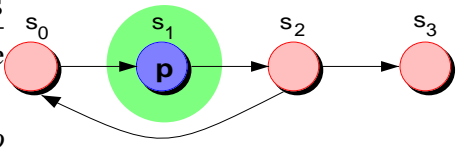

(b) primeira iteração

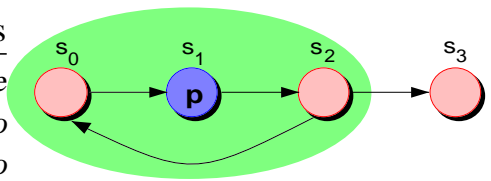

(d) terceira iteração

Figura 2.7: Funcionamento do algoritmo de ponto-fixo mínimo para $\exists \diamond p$.

Algoritmo de verificação para CTL. A seguir, apresentamos um algoritmo para verificação de propriedades especificadas em CTL. Esse algoritmo, denominado VM, recebe como entrada uma estrutura de Kripke $\mathcal{K}=\langle\mathcal{S}, \mathcal{T}, \mathcal{L}\rangle$ e uma fórmula CTL $\varphi$. A partir dessas entradas, o algoritmo computa o conjunto de estados $C \subseteq \mathcal{S}$ que não satisfazem a fórmula $\varphi$. Então, caso $C$ seja vazio, ele devolve sucesso; caso contrário, ele devolve $C$ como contra-exemplo. 
$\operatorname{VM}(\mathcal{K}, \varphi)$

$1 C \leftarrow \mathcal{S} \backslash \operatorname{SAT}(\varphi)$

2 se $C=\varnothing$ então devolva sucesso

3 devolva $C$

A operação básica no algoritmo VM é implementada pela função SAT, na qual a estrutura de Kripke $\mathcal{K}$ é tratada como variável global:

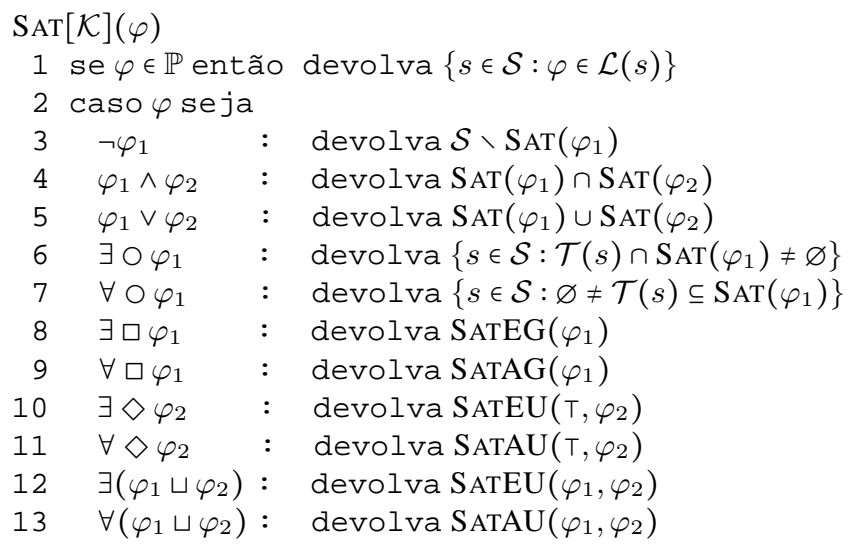

Nessa função, a semântica das fórmulas de CTL é obtida por indução estrutural. Operadores proposicionais e operadores temporais locais $(\exists \circ$ e $\forall \bigcirc)$ são tratados por meio de recursividade direta; enquanto operadores temporais globais ( $\exists \square, \forall \square, \exists \sqcup$ e $\forall \sqcup$ ) são tratados pelas seguintes funções especializadas para computação de pontos-fixos (que usam recursividade indireta):

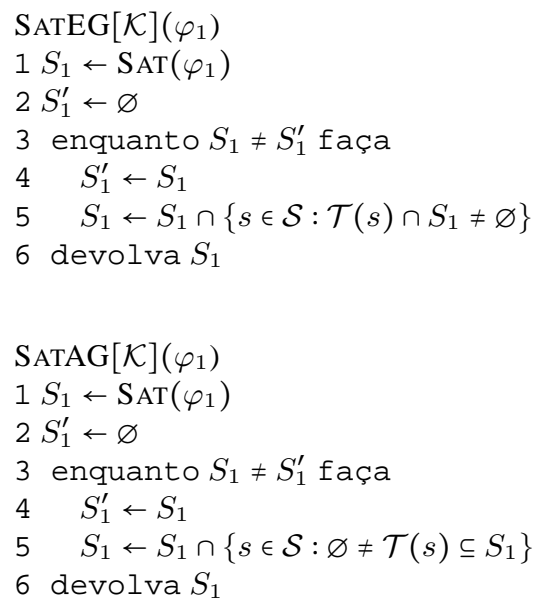


$\operatorname{SATEU}[\mathcal{K}]\left(\varphi_{1}, \varphi_{2}\right)$

$1 S_{1} \leftarrow \operatorname{SAT}\left(\varphi_{1}\right)$

$2 S_{2} \leftarrow \operatorname{SAT}\left(\varphi_{2}\right)$

$3 S_{2}^{\prime} \leftarrow \varnothing$

4 enquanto $S_{2} \neq S_{2}^{\prime}$ faça

$5 \quad S_{2}^{\prime} \leftarrow S_{2}$

$6 \quad S_{2} \leftarrow S_{2} \cup\left(\left\{s \in \mathcal{S}: \mathcal{T}(s) \cap S_{2} \neq \varnothing\right\} \cap S_{1}\right)$

7 devolva $S_{2}$

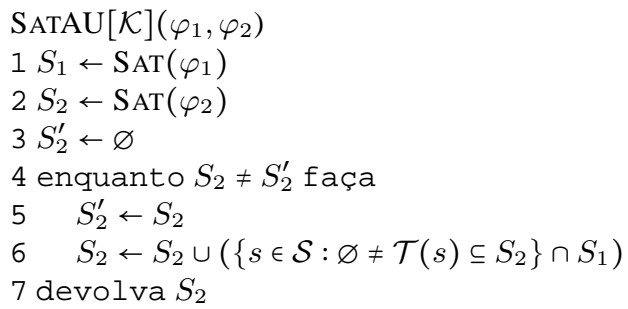

Note que as funções para computação de pontos-fixos máximos (SATEG e SATAG) e pontosfixos mínimos (SATEU e SATAU) são baseadas diretamente nas equivalências estabelecidas pelo Teorema 2.2. Como podemos ver, a caracterização de ponto-fixo para os operadores CTL possibilita a implementação de um algoritmo simples e efetivo para o problema de verificação de modelos. Esse algoritmo, porém, sofre do problema de "explosão de estados"; pois precisa construir explicitamente a estrutura de Kripke utilizada como modelo do sistema a ser verificado.

\subsection{Verificação de modelos simbólicos}

Verificação de modelos simbólicos [McMillan, 1992] é uma abordagem usada para controlar o problema de explosão de estados. Nessa abordagem, a estrutura de Kripke utilizada como modelo do sistema a ser verificado é representada, implicitamente, por meio de diagramas de decisão binária [Bryant, 1986], obtidos a partir da codificação proposicional de estados e transições.

Nessa seção, definimos a codificação proposicional de estados e transições; mostramos como, a partir dessa codificação, podemos obter diagramas de decisão binária representando uma estrutura de Kripke (i.e., modelo simbólico); e, finalmente, discutimos uma versão simbólica do algoritmo de verificação para CTL, que é capaz de manipular eficientemente esses diagramas. 


\subsubsection{Codificação proposicional}

O ponto de partida para a obtenção de um modelo simbólico é codificar estados e transições como fórmulas proposicionais.

Seja $\mathcal{K}=\langle\mathcal{S}, \mathcal{T}, \mathcal{L}\rangle$ uma estrutura de Kripke sobre $\mathbb{P}$. A codificação proposicional para um estado $s \in \mathcal{S}$, denotada por $\xi(s)$, é a fórmula

$$
\xi(s)=\bigwedge_{p \in \mathcal{L}(s)} p \wedge \bigwedge_{q \notin \mathcal{L}(s)} \neg q ;
$$

e a codificação proposicional para um conjunto de estados $X \subseteq \mathcal{S}$, denotada por $\xi(X)$, é a fórmula

$$
\xi(X)=\bigvee_{s \in X} \xi(s)
$$

Para codificar transições, precisamos de variáveis distintas para denotar propriedades que valem antes e depois das transições. Seja $\varphi$ uma fórmula proposicional. Usaremos a notação $[\varphi]^{\prime}$ para denotar a fórmula obtida por renomeação das variáveis proposicionais de $\varphi$, de modo que cada variável $p$ em $\varphi$ é substituída por outra variável $p^{\prime}$ correspondente. Essa função de renomeação deve ser bijetora, já que os símbolos $p$ e $p^{\prime}$ representam a mesma proposição, porém, em instantes de tempo consecutivos.

A codificação proposicional para uma transição $\left(s_{i}, s_{j}\right) \in \mathcal{T}$, denotada por $\xi\left(\left(s_{i}, s_{j}\right)\right)$, é a fórmula

$$
\xi\left(\left(s_{i}, s_{j}\right)\right)=\xi\left(s_{i}\right) \wedge\left[\xi\left(s_{j}\right)\right]^{\prime}
$$

e a codificação proposicional para uma relação de transições $\mathcal{T}$ é

$$
\xi(\mathcal{T})=\bigvee_{\left(s_{i}, s_{j}\right) \in \mathcal{T}} \xi\left(\left(s_{i}, s_{j}\right)\right)
$$

Exemplo 2.7 (Codificação proposicional da estrutura de Kripke). Considere a estrutura de Kripke $\mathcal{K}=\langle\mathcal{S}, \mathcal{L}, \mathcal{T}\rangle$ sobre $\mathbb{P}=\{p, q\}$, ilustrada na Figura 2.8. Os estados do conjunto $\mathcal{S}$ podem ser codificados como $\xi\left(s_{1}\right)=p \wedge q, \xi\left(s_{2}\right)=p \wedge \neg q$ e $\xi\left(s_{3}\right)=\neg p \wedge q$; e a relação de transição como $\xi(\mathcal{T})=\left(p \wedge q \wedge p^{\prime} \wedge \neg q^{\prime}\right) \vee\left(p \wedge q \wedge \neg p^{\prime} \wedge q^{\prime}\right) \vee\left(p \wedge \neg q \wedge \neg p^{\prime} \wedge q^{\prime}\right)$.

A seguir, mostramos como as fórmulas obtidas a partir da codificação proposicional de estados e transições podem ser convertidas para uma forma canônica, que permite a construção de uma representação simbólica eficiente para a estrutura de Kripke. 


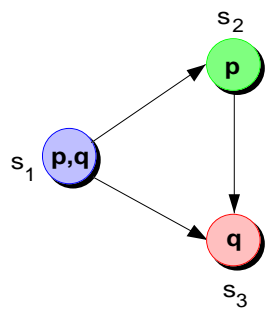

Figura 2.8: Estrutura de Kripke sobre o conjunto $\mathbb{P}=\{p, q\}$.

\subsubsection{Diagramas de decisão binária}

Sejam $\varphi$ uma fórmula proposicional e $\varphi[p:=c]$ a fórmula obtida pela substituição de toda ocorrência da variável $p$ em $\varphi$ pela constante $c \in\{\mathrm{T}, \perp\}$. A expansão de Shannon da fórmula $\varphi$, com relação à variável $p$, é dada pela seguinte equivalência:

$$
\varphi \equiv \varphi[p:=\top] \vee \varphi[p:=\perp] .
$$

Com base nessa equivalência, definimos o operador condicional ite (if-then-else) como:

$$
\operatorname{ite}(\phi, \alpha, \beta) \doteq(\phi \wedge \alpha) \vee(\neg \phi \wedge \beta),
$$

onde $\phi$ é denominado teste. Intuitivamente, se o teste é verdadeiro, a expressão ite $(\phi, \alpha, \beta)$ corresponde a $\alpha$; senão, corresponde a $\beta$.

Exemplo 2.8 (Equivalências). Todos os conectivos proposicionais podem ser expressos por meio do operador ite; veja:

- $\neg p=i t e(p, \perp, \top)$

- $p_{1} \wedge p_{2}=\operatorname{ite}\left(p_{1}, \top \wedge p_{2}, \perp \wedge p_{2}\right)=\operatorname{ite}\left(p_{1}, p_{2}, \perp\right)=\operatorname{ite}\left(p_{1}, \operatorname{ite}\left(p_{2}, \top, \perp\right), \perp\right)$

- $p_{1} \vee p_{2}=i \operatorname{te}\left(p_{1}, \top \vee p_{2}, \perp \vee p_{2}\right)=i \operatorname{te}\left(p_{1}, \top, p_{2}\right)=i \operatorname{te}\left(p_{1}, \mathrm{\top}, i t e\left(p_{2}, \top, \perp\right)\right)$

- $p_{1} \rightarrow p_{2}=i \operatorname{te}\left(p_{1}, \top \rightarrow p_{2}, \perp \rightarrow p_{2}\right)=i t e\left(p_{1}, p_{2}, \top\right)=i t e\left(p_{1}, i t e\left(p_{2}, \top, \perp\right), \top\right)$

Uma fórmula proposicional está na forma normal condicional se e só se contém apenas o operador ite, todos os testes são realizados sobre variáveis proposicionais e todas as variáveis proposicionais ocorrem apenas como testes [Andersen, 1997]. Toda fórmula proposicional $\varphi$ pode ser convertida para a forma normal condicional. Essa conversão é feita do seguinte modo: enquanto houver uma variável proposicional $p \in \varphi$ que não esteja sendo usada como teste, reescreva $\varphi$ como ite $(p, \varphi[p:=\top], \varphi[p:=\perp])$. 
Exemplo 2.9 (Conversão para forma normal condicional). Veja a conversão da fórmula $\left(p_{1} \vee\right.$ $\left.p_{2}\right) \wedge\left(p_{2} \vee p_{3}\right)$ para a forma normal condicional:

$$
\begin{aligned}
& \left(p_{1} \vee p_{2}\right) \wedge\left(p_{2} \vee p_{3}\right) \\
& =\operatorname{ite}\left(p_{1},\left(\top \vee p_{2}\right) \wedge\left(p_{2} \vee p_{3}\right),\left(\perp \vee p_{2}\right) \wedge\left(p_{2} \vee p_{3}\right)\right) \\
& =\operatorname{ite}\left(p_{1}, p_{2} \vee p_{3}, p_{2}\right) \\
& =\operatorname{ite}\left(p_{1}, i \operatorname{ite}\left(p_{2}, \top \vee p_{3}, \perp \vee p_{3}, i \operatorname{te}\left(p_{2}, \top, \perp\right)\right)\right. \\
& =\operatorname{ite}\left(p_{1}, i \operatorname{te}\left(p_{2}, \top, p_{3}, i \operatorname{tie}\left(p_{2}, \top, \perp\right)\right)\right. \\
& =\operatorname{ite}\left(p_{1}, i \operatorname{ite}\left(p_{2}, \top, i t e\left(p_{3}, \top, \perp\right), i t e\left(p_{2}, \top, \perp\right)\right)\right.
\end{aligned}
$$

Árvore de decisão binária. A forma normal condicional de uma fórmula proposicional descreve um grafo, denominado árvore de decisão binária, que define o valor dessa fórmula sob toda interpretação possível de suas variáveis. Por exemplo, na Figura 2.9-a, podemos ver a árvore de decisão binária correspondente à fórmula $\left(p_{1} \vee p_{2}\right) \wedge\left(p_{2} \vee p_{3}\right)$, cuja forma normal condicional é ite $\left(p_{1}, i \operatorname{te}\left(p_{2}, \mathrm{\top}\right.\right.$, ite $\left(p_{3}, \top, \perp\right)$, ite $\left.\left(p_{2}, \top, \perp\right)\right)$.

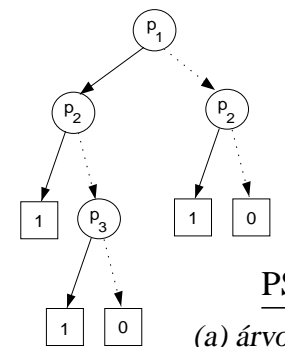

(a) árvore de decisão binária

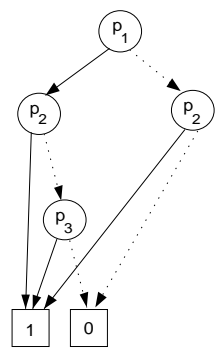

(b) diagrama de decisão binária

Figura 2.9: Árvore e diagrama de decisão binária para a fórmula $\left(p_{1} \vee p_{2}\right) \wedge\left(p_{2} \vee p_{3}\right)$.

Numa árvore de decisão binária, as folhas são rotuladas com as constantes 0 e 1 (denotando $\perp$ e T, respectivamente) e os demais nós são rotulados com variáveis proposicionais. Cada nó interno $p_{i}$ tem um filho esquerdo $\left(p_{i}=\mathrm{T}\right)$, indicado por uma linha contínua, e um filho direito $\left(p_{i}=\perp\right)$, indicado por uma linha pontilhada.

Diagrama de decisão binária ordenado e reduzido. Embora as árvores de decisão sejam muito úteis para representar fórmulas proposicionais, freqüentemente, elas apresentam muita redundância. Parte dessa redundância pode ser eliminada por meio do compartilhamento de subgrafos isomorfos. Quando esse compartilhamento é feito, a árvore é transformada num grafo 
orientado acíclico, denominado diagrama de decisão binária (BDD - BINARY DECISION DIAGRAM) [Bryant, 1986]. Por exemplo, o resultado do compartilhamento de subgrafos isomorfos na árvore de decisão da fórmula $p_{1} \wedge\left(p_{2} \vee p_{3}\right) \wedge\left(\neg p_{2} \vee p_{3}\right)$ (Figura 2.10-a) pode ser visto na Figura 2.10-b.

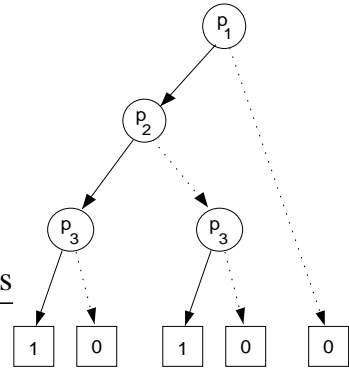

(a) grafo original

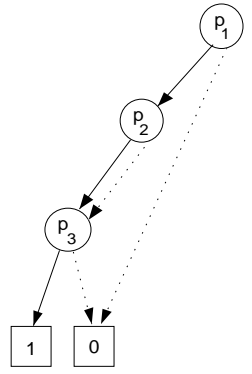

(b) compartilha subgrafos

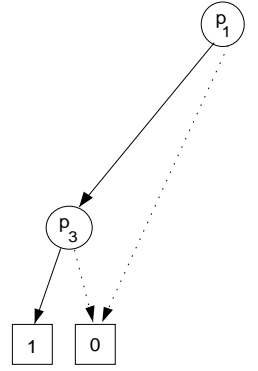

(c) elimina testes redundantes

Figura 2.10: Compartilhamento de grafos isomorfos e eliminação de testes redundantes.

Quando a ordem das variáveis de teste em todos os caminhos que levam da raiz até uma folha da árvore é sempre a mesma, o grafo obtido pelo compartilhamento é denominado diagrama de decisão binária ordenado (OBDD - ORDERED BDD). Às vezes, após o compartilhamento, alguns testes tornam-se redundantes. Para eliminar um teste redundante, basta excluir o nó que o representa e redirecionar todo arco de entrada desse nó para o seu filho (Figura 2.10-c). Quando todos os testes redundantes num diagrama de decisão binária ordenado são eliminados, o grafo resultante é denominado diagrama de decisão binária ordenado e reduzido (ROBDD - REDUCED OBDD). Uma propriedade importante sobre ROBDDs é que duas fórmulas equivalentes têm sempre o mesmo ROBDD correspondente [Bryant, 1992], isso possibilita um teste de terminação bastante eficiente em algoritmos que computam pontos-fixos [Andersen, 1997]. Cabe ainda salientar que, na literatura, é comum o uso da sigla BDD como sinônimo de ROBDD.

Algoritmos para manipulação de ROBDDs. Em termos de estruturas de dados, um ROBDD é uma tabela $T: n \mapsto\langle v, t, f\rangle$, que associa a cada identificador $n$ um nó com variável de teste $v$, filho esquerdo $t$ e filho direito $f$ [Pereira \& Barros, 2007a]. Ademais, devido ao compartilhamento de subgrafos, a tabela $T$ admite uma inversa $T^{-1}:\langle v, t, f\rangle \mapsto n$, mapeando nós em identificadores, utilizada para garantir que os diagramas sejam reduzidos. Também assumimos que $T(n)=T^{-1}(\langle v, t, f\rangle)=$ nulo, sempre que $(n,\langle v, t, f\rangle) \notin T$. Nessas tabelas, os iden- 
tificadores 0 e 1 são reservados para os nós folhas; as variáveis proposicionais $p_{1}, p_{2}, \ldots$ são representadas pelos índices $1,2, \ldots$; e a ordem em que as variáveis são testadas é definida pelos seus índices. Para facilitar a representação dos algoritmos, tratamos a tabela $T$ como uma variável global e usamos $|T|$ para denotar o número de tuplas nessa tabela.

- Iniciação da estrutura: O algoritmo InICIA recebe uma entrada $m$, indicando o número de variáveis proposicionais existentes na fórmula a ser representada, e inicia a tabela $T$ com duas tuplas especiais, representando os nós terminais 0 e 1 . Para um tratamento uniforme, esses nós terminais são associados à variável $p_{m+1}$.

$\operatorname{INICIA}[T](m)$

$1 T \leftarrow\{(0,\langle m+1$, nulo, nulo $\rangle),(1,\langle m+1$, nulo, nulo $\rangle)\}$

- Inserção de nós: Para inserir um nó na tabela, usamos o algoritmo INSERE. Caso o nó seja um teste redundante, o algoritmo simplesmente devolve o identificador de seu filho; caso o nó já tenha sido criado anteriormente, o algoritmo devolve seu identificador; e, finalmente, caso o nó seja novo, o algoritmo insere na tabela uma nova tupla para representar esse nó e devolve seu identificador.

$\operatorname{INSERE}[T](v, t, f)$

1 se $t=f$ então devolva $t$

$2 n \leftarrow T^{-1}(\langle v, t, f\rangle)$

3 se $n=$ nulo então

$n \leftarrow|T|$

$T \leftarrow T \cup\{(n,\langle v, t, f\rangle)\}$

7 devolva $n$

- Construção do diagrama: $\mathrm{O}$ algoritmo Constrói recebe uma fórmula ite $\left(v, \varphi_{t}, \varphi_{f}\right)$, na forma normal condicional, cria uma tabela com os nós do diagrama de decisão para essa fórmula e devolve o identificador para o nó raiz desse diagrama.

CONSTRÓi $\left(i t e\left(v, \varphi_{t}, \varphi_{f}\right)\right)$

1 se $\varphi_{t}, \varphi_{f} \in\{0,1\}$ então devolva $\operatorname{InSere}\left(v, \varphi_{t}, \varphi_{f}\right)$

2 se $\varphi_{f} \in\{0,1\}$ então devolva $\operatorname{InSeRe}\left(v, \operatorname{Constró}\left(\varphi_{t}\right), \varphi_{f}\right)$

3 se $\varphi_{t} \in\{0,1\}$ então devolva $\operatorname{Insere}\left(v, \varphi_{t}, \operatorname{Constrói}\left(\varphi_{f}\right)\right)$

4 devolva Insere $\left(v, \operatorname{Constrói~}\left(\varphi_{t}\right), \operatorname{Constrói~}\left(\varphi_{f}\right)\right)$

- Operações lógicas: As operações lógicas entre fórmulas são implementadas pelo algoritmo genérico APLICA, que baseia-se na seguinte equivalência:

$$
\operatorname{ite}\left(v, \varphi_{t}, \varphi_{f}\right) \star \operatorname{ite}\left(v, \varphi_{t}^{\prime}, \varphi_{f}^{\prime}\right) \equiv i t e\left(v, \varphi_{t} \star \varphi_{t}^{\prime}, \varphi_{f} \star \varphi_{f}^{\prime}\right), \operatorname{para} \star \epsilon\{\wedge, \vee, \rightarrow\}
$$


Assim, para efetuarmos uma operação lógica entre duas fórmulas, basta aplicarmos essa transformação, recursivamente, a partir das raízes dos diagramas dessas fórmulas. Para maior eficiência, o algoritmo a seguir utiliza memoização $o^{3}$ [Cormen et al., 2001], implementada por meio da tabela $M$.

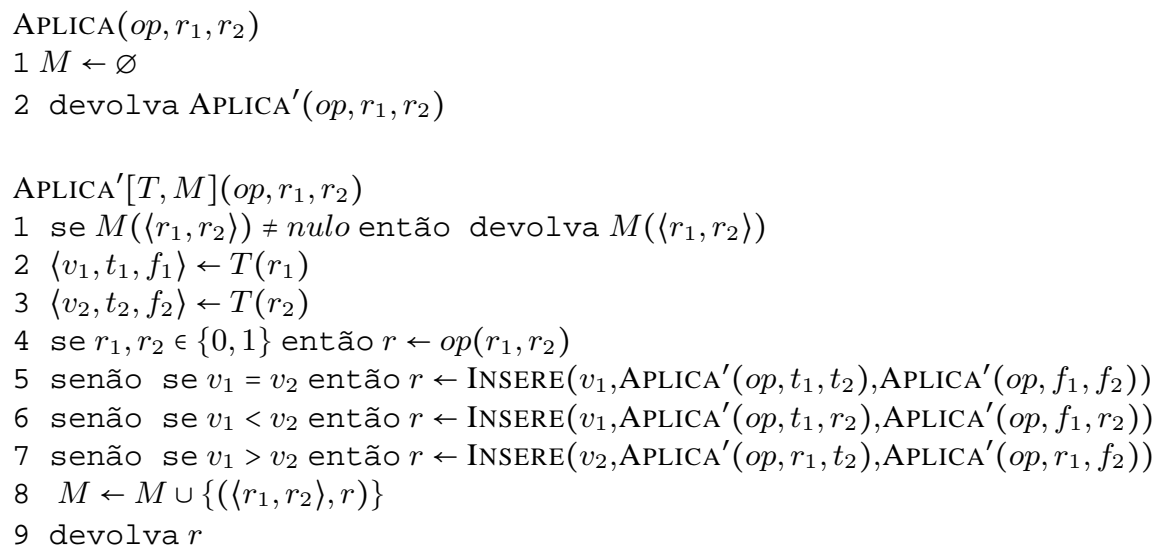

\subsubsection{Algoritmo para verificação de modelos simbólicos}

A idéia básica do algoritmo de verificação de modelos simbólicos é empregar ROBDDs para representar funções características de conjuntos de estados e transições da estrutura de Kripke, que serve de modelo para o sistema a ser verificado [McMillan, 1992]. Se $\Phi$ é uma relação $n$-ária sobre $\{0,1\}$, então $\Phi$ pode ser representada pelo ROBDD de sua função característica:

$$
f_{\Phi}\left(x_{1}, \ldots, x_{n}\right)=1 \Leftrightarrow\left(x_{1}, \ldots, x_{n}\right) \in \Phi
$$

Para que relações complexas possam ser construídas, é conveniente estender a lógica proposicional de modo a permitir quantificação sobre os valores das proposições:

$$
\begin{aligned}
& \forall p \varphi \equiv \varphi[p:=\top] \wedge \varphi[p:=\perp] \\
& \exists p \varphi \equiv \varphi[p:=\top] \vee \varphi[p:=\perp]
\end{aligned}
$$

Fórmulas proposicionais quantificadas têm o mesmo poder expressivo das fórmulas proposicionais ordinárias; porém, são mais concisas. Toda fórmula proposicional quantificada determina uma relação $n$-ária sobre $\{T, \perp\}$. Dado um diagrama de decisão binária para uma

\footnotetext{
${ }^{3}$ Técnica de programação dinâmica sob demanda, isso permite que o algoritmo consuma tempo polinomial.
} 
fórmula proposicional $\varphi$, é fácil construir os diagramas para as fórmulas $\forall p \varphi \mathrm{e} \exists p \varphi$, usando as equivalências (2.9) e (2.10). Na prática, porém, precisamos de algoritmos especiais para tratar eficientemente os quantificadores.

Produto relacional. Particularmente, no algoritmo de verificação que apresentamos nessa seção, quantificadores ocorrem apenas em produtos relacionais, que têm a seguinte forma:

$$
\exists \vec{p}\left[\varphi_{1}(\vec{p}) \wedge \varphi_{2}(\vec{p})\right],
$$

onde $\vec{p}$ é um vetor de variáveis proposicionais. Isso possibilita um tratamento muito eficiente de quantificadores. De fato, o algoritmo PRODREL, apresentado a seguir, realiza essa operação sobre os diagramas de decisão binária das fórmulas $\varphi_{1}(\vec{p})$ e $\varphi_{2}(\vec{p})$, cujas raízes são $r_{1}$ e $r_{2}$, sem ter que construir o diagrama para a fórmula $\varphi_{1}(\vec{p}) \wedge \varphi_{2}(\vec{p})$.

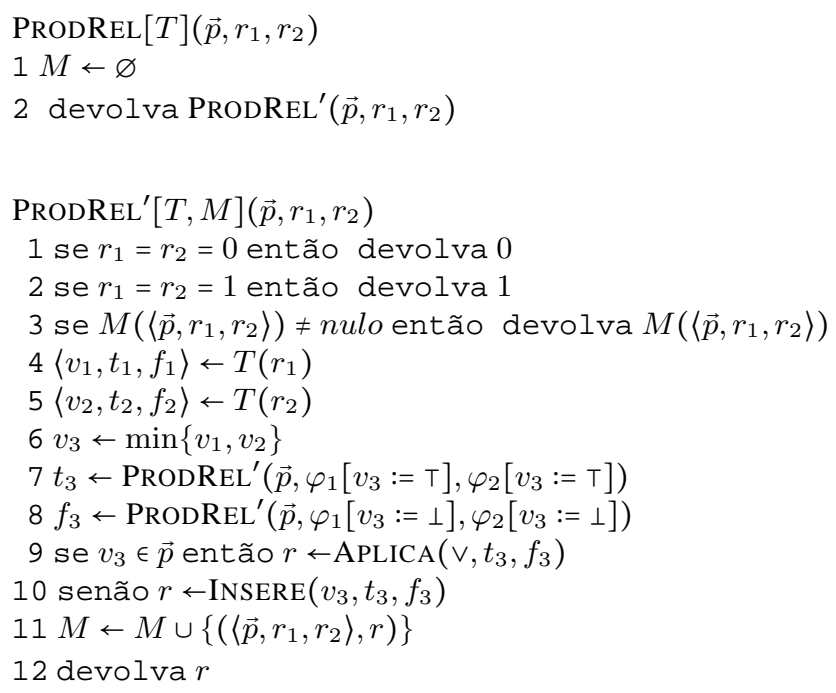

Estados e transições como ROBDDs. Dado um conjunto $A$, a função característica $A(x)=$ $x \in A$ permite identificar todo elemento $x$ de $A$. Um estado pode ser representado por um vetor $\vec{p}=\left(p_{1}, \ldots, p_{k}\right)$, onde $k$ é o número de proposições que descrevem os estados na estrutura de Kripke. Assim, um conjunto de estados $S$ pode ser representado por uma função característica $S(\vec{p})$, codificada como um ROBDD. Analogamente, a função característica $T\left(\left(\vec{p}, \vec{p}^{\prime}\right)\right)$ pode ser usada para representar a relação de transição. 
Exemplo 2.10 (Estados sucessores). Considere novamente a estrutura de Kripke da Figura 2.8, cuja relação de transição $\mathcal{T}$ pode ser representada pela seguinte função característica:

$$
\begin{aligned}
T\left((p, q),\left(p^{\prime}, q^{\prime}\right)\right)= & \left(p \wedge q \wedge p^{\prime} \wedge \neg q^{\prime}\right) \vee & & / /\left(s_{1}, s_{2}\right) \\
& \left(p \wedge q \wedge \neg p^{\prime} \wedge q^{\prime}\right) \vee & & / /\left(s_{1}, s_{3}\right) \\
& \left(p \wedge \neg q \wedge \neg p^{\prime} \wedge q^{\prime}\right) & & / /\left(s_{2}, s_{3}\right)
\end{aligned}
$$

Usando produto relacional, o conjunto de estados sucessores de $s_{1}$, denotado por $\mathcal{T}\left(s_{1}\right)$, pode ser obtido da seguinte maneira:

$$
\begin{aligned}
\mathcal{T}\left(s_{1}\right) & =\left(\exists p, q \cdot\left(\xi\left(s_{1}\right) \wedge T\left((p, q),\left(p^{\prime}, q^{\prime}\right)\right)\right)\left[p^{\prime}:=p, q^{\prime}:=q\right]\right. \\
& =\left(\exists p, q \cdot\left((p \wedge q) \wedge T\left((p, q),\left(p^{\prime}, q^{\prime}\right)\right)\right)\left[p^{\prime}:=p, q^{\prime}:=q\right]\right. \\
& =\left(\exists p, q \cdot\left(\left(p \wedge q \wedge p^{\prime} \wedge \neg q^{\prime}\right) \vee\left(p \wedge q \wedge \neg p^{\prime} \wedge q^{\prime}\right)\right)\right)\left[p^{\prime}:=p, q^{\prime}:=q\right] \\
& \left.=\left(p^{\prime} \wedge \neg q^{\prime} \vee \neg p_{1}^{\prime} \wedge q^{\prime}\right)\right)\left[p^{\prime}:=p, q^{\prime}:=q\right] \\
& =(p \wedge \neg q \vee \neg p \wedge q)) \\
& =\left\{\xi\left(s_{2}\right), \xi\left(s_{3}\right)\right\}
\end{aligned}
$$

Note que a quantificação existencial abstrai o estado origem da transição e a renomeação restaura os nomes das variáveis proposicionais que descrevem o estado corrente. Assim, para obter estados predecessores, basta quantificar existencialmente as variáveis de estado sucessor (ou seja, as variáveis representada pelo vetor $\vec{p}^{\prime}$ ).

O algoritmo de verificação simbólica VMS. O algoritmo de verificação simbólica VMS recebe como entrada um ROBDD $k$, representando uma estrutura de Kripke $\mathcal{K}$, e uma fórmula CTL $\varphi$, especificando a propriedade a ser verificada nessa estrutura. Através de uma chamada à função $\operatorname{SATS}(\varphi)$, o algoritmo obtém um ROBDD $s$ representando o conjunto de estados que satisfazem a fórmula $\varphi$. Em seguida, usando a equivalência $\neg s \equiv s \rightarrow \perp$, ele obtém um novo ROBDD $c$, representando o conjunto de estados que não satisfazem a fórmula $\varphi$. Então, caso $c$ seja o ROBDD da fórmula $\perp$ (i.e., o conjunto vazio), o algoritmo devolve sucesso; caso contrário, ele devolve $c$ como contra-exemplo.

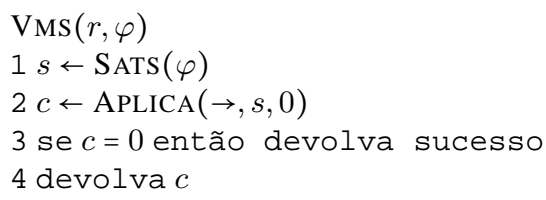


Como na versão enumerativa, a função SATS pode ser definida indutivamente sobre a estrutura da fórmula $\varphi$. Se $\varphi$ for uma fórmula proposicional, então $\operatorname{SATS}(\varphi)$ deve simplesmente devolver o ROBDD para $\varphi$; caso contrário, a função precisa chamar funções auxiliares especializadas: SATSEX, SATSEG e SATSEU.

A função SATSEX é implementada diretamente como um produto relacional, sendo suficiente encontrar os predecessores daqueles estados onde a fórmula sendo verificada é satisfeita:

$$
\operatorname{SATSEX}(\varphi(\vec{p}))=\exists \vec{p}^{\prime}\left[\varphi\left(\vec{p}^{\prime}\right) \wedge r\left((\vec{p}),\left(\vec{p}^{\prime}\right)\right)\right]
$$

As outras duas funções (SATSEG e SATSEU) são baseadas na caracterização de ponto-fixo dos operadores CTL, além do produto relacional:

$$
\begin{gathered}
\operatorname{Satseg}(\varphi(\vec{p}))=\nu Y(\vec{p}) \cdot[\varphi(\vec{p}) \wedge \operatorname{SAtseX}(Y(\vec{p}))] \\
\operatorname{SatsEU}\left(\varphi_{1}(\vec{p}), \varphi_{2}(\vec{p})\right)=\mu Y(\vec{p}) \cdot\left[\varphi_{2}(\vec{p}) \vee\left(\varphi_{1}(\vec{p}) \wedge \operatorname{SATSEX}(Y(\vec{p}))\right)\right]
\end{gathered}
$$

Note que essas funções auxiliares recebem fórmulas proposicionais como argumentos, enquanto o algoritmo VMS recebe uma fórmula temporal. Os casos de fórmulas proposicionais com operadores lógicos usuais são tratados pelo algoritmo APLICA (pág. 32). E os demais operadores temporais da lógica CTL podem ser tratados empregando-se as seguintes equivalências:

- $\forall \circ \varphi \equiv \neg \exists \bigcirc \neg \varphi$

- $\forall \square \varphi \equiv \neg \exists \diamond \neg \varphi$

- $\forall\left(\varphi_{1} \sqcup \varphi_{2}\right) \equiv \neg\left(\exists\left(\neg \varphi_{2} \sqcup\left(\neg \varphi_{1} \wedge \neg \varphi_{2}\right)\right) \vee \exists \square \neg \varphi_{2}\right)$

\subsection{Sumário}

Nesse capítulo, mostramos que problema de verificação de modelos [Müller-Olm et al., 1999] consiste, essencialmente, em verificar se uma dada estrutura de Kripke [Kripke, 1963], representando o modelo formal de um sistema, é também um modelo semântico para uma fórmula temporal, representando uma certa propriedade a ser verificada nesse sistema.

Também apresentamos uma caracterização de ponto-fixo [Tarski, 1955] para os operadores da lógica temporal de tempo ramificado CTL [Clarke \& Emerson, 1982], a partir da qual derivamos um algoritmo para verificação de modelos, que denominamos VM. 
Vimos também que o algoritmo apresentado para verificação de modelos, apesar de efetivo, sofre do problema de "explosão de estados". Então, como uma forma de controlar os efeitos desse problema, apresentamos também uma abordagem simbólica para verificação de modelos [McMillan, 1992]. Nessa abordagem, conjuntos de estados e transições são representados implicitamente por meio de diagramas de decisão binária ordenados e reduzidos (ROBDDs) [Bryant, 1992], obtidos a partir de uma codificação proposicional dos estados e transições na estrutura de Kripke. Finalmente, usando esses diagramas, apresentamos um algoritmo bastante eficiente para verificação de modelos simbólicos, que denominamos VMs. Esse algoritmo é a base para uma implementação eficiente dos métodos de planejamento que propomos nessa tese.

No próximo capítulo, mostraremos como o arcabouço de verificação de modelos pode ser empregado para resolver problemas de planejamento sob incerteza qualitativa. 


\section{Capítulo 3}

\section{Planejamento baseado em verificação de modelos}

\subsection{O arcabouço de verificação de modelos}

Como mostramos no Capítulo 2, dados um modelo $\mathcal{K}$ e uma fórmula $\varphi$, verificação de modelos consiste em resolver o problema $\mathcal{K} \stackrel{?}{\vDash} \varphi$. Quando o arcabouço de verificação de modelos é empregado no contexto de planejamento automatizado [Giunchiglia \& Traverso, 1999], o modelo $\mathcal{K}$ descreve a dinâmica do ambiente de planejamento, enquanto a fórmula $\varphi$ descreve a meta do agente nesse ambiente. Nesse caso, além de $\mathcal{K}$ e $\varphi$, o planejador recebe também o estado inicial $s_{0}$ do ambiente. Então, se $\left(\mathcal{K}, s_{0}\right) \vDash \varphi$, o planejador devolve um plano, i.e., uma política de comportamento que permite ao agente atingir sua meta; senão, ele devolve fracasso (Figura 3.1).

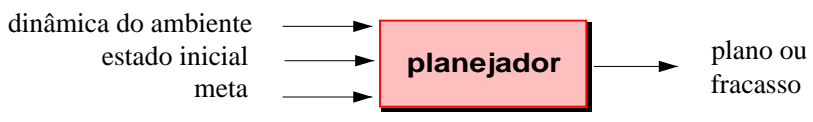

Figura 3.1: Planejamento baseado em verificação de modelos

Note, porém, que há uma diferença significativa entre verificadores e planejadores: enquanto um verificador precisa verificar a validade da propriedade $\varphi$ em todos os estados do modelo, em geral, um planejador precisa garantir a validade dessa propriedade apenas no grafo de transições induzido pelas ações incluídas no plano sintetizado como solução (ou seja, num “submodelo"). 


\subsection{Domínio de planejamento}

Sejam $\mathbb{P} \neq \varnothing$ um conjunto finito de proposições atômicas, que denotam propriedades de estados de um ambiente de planejamento, e $\mathbb{A} \neq \varnothing$ um conjunto finito de ações, que representam as habilidades do agente nesse ambiente. Um domínio de planejamento é um modelo formal para a dinâmica do ambiente do agente e, como os conjuntos $\mathbb{P}$ and $\mathbb{A}$ são dependentes do ambiente específico considerado, o par $(\mathbb{P}, \mathbb{A})$ é denominado assinatura do domínio de planejamento.

Definição 3.1. Um domínio de planejamento qualitativo com assinatura $(\mathbb{P}, \mathbb{A})$ é definido por uma tupla $\mathcal{D}=\langle\mathcal{S}, \mathcal{L}, \mathcal{T}\rangle$, onde:

- $\mathcal{S} \neq \varnothing$ é um conjunto finito de estados;

- $\mathcal{L}: \mathcal{S} \mapsto 2^{\mathbb{P}}$ é uma função de rotulação de estados;

- $\mathcal{T}: \mathcal{S} \times \mathbb{A} \mapsto 2^{\mathcal{S}}$ é uma função de transição de estados.

O conjunto $\mathcal{S}$ contém todos os possíveis estados do ambiente de planejamento e, a cada estado $s \in \mathcal{S}$, a função de rotulação de estados $\mathcal{L}$ associa um conjunto de proposições $\mathcal{L}(s) \in 2^{\mathbb{P}}$. Assumimos que, para todo estado $s \in \mathcal{S}$, o conjunto $\mathcal{L}(s)$ é consistente e $T \in \mathcal{L}(s)$.

A incerteza que o agente tem a respeito do comportamento da natureza é modelada pela função de transição $\mathcal{T}$. Dados um estado $s \in \mathcal{S}$ e uma ação $a \in \mathbb{A}$, o conjunto de $a$-sucessores de $s$, denotado por $\mathcal{T}(s, a)$, é o conjunto de todos os estados que podem ser diretamente alcançados pela execução da ação $a$ no estado $s$. Particularmente, assumimos que $\mathcal{T}(s, a)=\varnothing$, se a ação $a$ não pode ser executada no estado $s$. Ademais, quando $a$ é executada em $s$, apenas um dos estados em $\mathcal{T}(s, a)$ será, de fato, o estado corrente. Porém, por se tratar de um modelo de incerteza qualitativo, não sabemos com que probabilidade cada um dos estados no conjunto $\mathcal{T}(s, a)$ poderá ser o próximo estado corrente do ambiente.

Um domínio de planejamento qualitativo com assinatura $(\mathbb{P}, \mathbb{A})$ pode ser representado por um grafo de transições cujos estados são rotulados com subconjuntos de $\mathbb{P}$ e cujas transições são rotuladas com elementos de $\mathbb{A}$.

Exemplo 3.1 (Grafo de transições). Na Figura 3.2, podemos ver o grafo de transições para um domínio de planejamento qualitativo com assinatura $(\mathbb{P}, \mathbb{A})$, onde $\mathbb{P}=\{p, q, r\}$ e $\mathbb{A}=\{a, b, c\}$. Por exemplo, considerando o estado $s_{0}$ nesse domínio, temos $\mathcal{L}\left(s_{0}\right)=\{\top\}, \mathcal{T}\left(s_{0}, a\right)=\left\{s_{1}, s_{2}\right\}$ $e \mathcal{T}\left(s_{0}, b\right)=\left\{s_{3}\right\}$. Observe que, ao contrário da ação $b$, que tem efeito determinístico quando executada em $s_{0}$, a ação a tem efeito incerto quando executada nesse mesmo estado. 


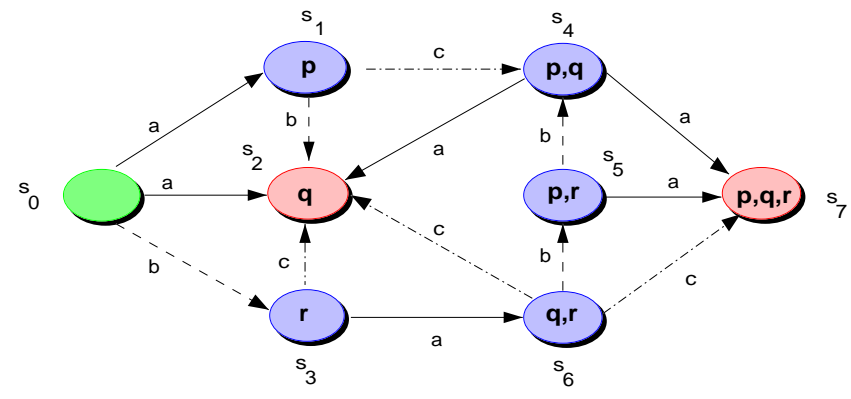

Figura 3.2: Domínio com assinatura $(\mathbb{P}, \mathbb{A})$, sendo $\mathbb{P}=\{p, q, r\}$ e $\mathbb{A}=\{a, b, c\}$.

Embora um domínio de planejamento possa ser formalmente especificado por meio de um grafo de transições (modelo explícito), na prática, isso pode ser inviável. Como o número de estados num domínio de planejamento é exponencial no número de proposições usadas na descrição dos estados do ambiente, em geral, é praticamente impossível enumerar todos os estados e transições explicitamente. Ademais, é muito mais simples, intuitivo e seguro especificar ações por meio de precondições e efeitos, do que por meio da enumeração explícita de estados e transições. Assim, em aplicações práticas de planejamento, domínios são normalmente especificados através do uso de linguagens de alto-nível, especializadas para descrição de ações (e.g., StRIPS [Fikes \& Nilsson, 1990], AdL [Pednault, 1989] e PdDL [McDermott, 1998]).

Na próxima seção, mostramos como um modelo explícito de domínio pode ser obtido a partir de uma especificação de ações numa linguagem de alto-nível. Isso permitirá que a formalização que apresentamos no restante dessa tese seja baseada apenas em modelos explícitos (que podem ser obtidos, automaticamente, a partir de especificações de alto-nível). Desta forma, ainda que os conceitos apresentados na Seção 3.3 sejam bastante relevantes para aplicações práticas de planejamento, eles não são essenciais para a compreensão dos próximos capítulos.

\subsection{Linguagem $\mathcal{A R}$}

$\mathcal{A R}$ [Giunchiglia et al., 1997] é uma linguagem formal para descrição de ações que, além de uma sintaxe intuitiva e uma semântica bem definida, incorpora uma solução para o problema de persistência temporal ${ }^{1}$ na presença de efeitos incertos e indiretos (i.e., não-determinismo e ramificação). Em $\mathcal{A R}$, as proposições que denotam propriedades dos estados do ambiente são

\footnotetext{
${ }^{1}$ Frame problem [Shanahan, 1997].
} 
denominadas fluentes. Um fluente cujo valor pode ser modificado apenas em decorrência da execução de ações do agente é denominado inerte; enquanto um fluente cujo valor pode ser modificado em conseqüência da ocorrência de um evento exógeno é denominado não-inerte.

\subsubsection{Sintaxe}

Formalmente, $\mathcal{A R}$ é uma família de linguagens; cada uma delas caracterizada por um conjunto de fluentes, parte dos quais devem ser inertes, e por um conjunto de ações.

Uma fórmula atômica em $\mathcal{A R}$ é uma expressão da forma

$$
(p \text { is } x)
$$

onde $p$ é um fluente e $x \in \operatorname{Dom}(p)$. Quando $\operatorname{Dom}(p)=\{\mathrm{T}, \perp\}$, uma fórmula ( $p$ is $\mathrm{T}$ ) pode ser abreviada por $p$ (analogamente, uma fórmula ( $p$ is $\perp$ ) pode ser abreviada por $\neg p$ ). Uma fórmula é uma combinação proposicional de fórmulas atômicas.

Uma linguagem $\mathcal{A R}$ permite sentenças das seguintes formas (onde $a$ é uma ação, $p$ é um fluente inerte e $\varphi$ é uma fórmula proposicional):

$$
\begin{gathered}
a \text { causes } \varphi^{\prime} \text { if } \varphi \\
a \text { possibly changes } p \text { if } \varphi \\
\text { always } \varphi
\end{gathered}
$$

Intuitivamente, uma sentença da forma (3.2) estabelece que a execução da ação $a$, em um estado que satisfaz $\varphi$, causa necessariamente uma transição para um estado que satisfaz $\varphi^{\prime}$; uma sentença da forma (3.3) estabelece que a execução da ação $a$, em um estado que satisfaz $\varphi$, causa possivelmente uma transição para um estado onde o valor do fluente $p$ é modificado; e, finalmente, uma sentença da forma (3.4) estabelece que a fórmula $\varphi$ deve ser satisfeita em todo estado do domínio de planejamento. Uma sentença da forma ( $a$ causes $\varphi^{\prime}$ if T) pode ser abreviada por $\left(a\right.$ causes $\left.\varphi^{\prime}\right)$. Analogamente, ( $a$ causes $\perp$ if $\neg \varphi$ ) pode ser abreviada por ( $a$ has preconditions $\varphi$ ), estabelecendo que a ação $a$ só pode ser executada em estados que satisfazem a precondição $\varphi$. Um conjunto de sentenças das formas (3.2), (3.3) e (3.4) é denominado especificação de domínio. 


\subsubsection{Semântica}

A semântica de uma especificação de domínio é representada por uma função de transição de estados $\mathcal{T}$ correspondente. Intuitivamente, $\mathcal{T}(s, a)$ denota o conjunto de estados que podem resultar da execução da ação $a$ no estado $s$.

Estados. Uma valoração é uma função $\sigma$ que associa a cada fluente $p$ um elemento $x \in$ $\operatorname{Dom}(p)$. Uma valoração $\sigma$ é estendida para fórmulas atômicas da seguinte maneira:

$$
\sigma(p \text { is } x)= \begin{cases}\top & \text { se } \sigma(p)=x \\ \perp & \text { caso contrário }\end{cases}
$$

A extensão de uma valoração $\sigma$ para fórmulas arbitrárias pode ser definida indutivamente sobre a estrutura dessas fórmulas, usando a semântica padrão dos conectivos da lógica proposicional.

Definição 3.2. Seja $\mathcal{D}$ uma especificação de domínio e $\sigma$ uma valoração. Então, $\sigma$ é um estado em $\mathcal{D}$ se e só se, para toda sentença da forma (always $\varphi$ ) em $\mathcal{D}, \sigma(\varphi)=T$.

Em outras palavras, um estado é uma valoração que satisfaz todas as sentenças da forma (always $\varphi$ ) existentes numa especificação de domínio $\mathcal{D}$.

Transições. Para uma ação $a$ e um estado $s$, seja $\mathcal{T}_{0}(s, a)$ o conjunto de estados $s^{\prime}$ tais que, para cada sentença da forma ( $a$ causes $\varphi^{\prime}$ if $\varphi$ ) em $\mathcal{D}$, temos $s^{\prime}\left(\varphi^{\prime}\right)=$ T sempre que $s(\varphi)=$ T. Ou seja, $\mathcal{T}_{0}(s, a)$ é o conjunto de estados que satisfazem a fórmula $\varphi^{\prime}$ (efeito de $a$ ), sempre que o estado $s$ satisfaz a fórmula $\varphi$ (precondição de $a$ ). O conjunto $\mathcal{T}(s, a)$ é definido como o subconjunto de $\mathcal{T}_{0}(s, a)$ que contém apenas "estados bem próximos" de $s$.

Para tornar essa noção de "estados bem próximos" mais precisa, vamos usar $\mathcal{N}_{a}\left(s, s^{\prime}\right)$ para denotar o conjunto de fórmulas atômicas cujos valores em $s^{\prime}$ são resultantes da execução da ação $a$ no estado $s$. Formalmente, $\mathcal{N}_{a}\left(s, s^{\prime}\right)$ é o conjunto de fórmulas da forma

$$
p \text { is } s^{\prime}(p)
$$

tais que:

- (i) $p$ é um fluente inerte e $s^{\prime}(p) \neq s(p)$; ou

- (ii) existe em $\mathcal{D}$ uma sentença da forma ( $a$ possibly changes $p$ if $\varphi$ ) e $s(\varphi)=T$. 
A condição (i) vale se (3.6) é um fato que torna-se verdadeiro quando a execução da ação $a$ no estado $s$ resulta no estado $s^{\prime}$ (isso significa que o conjunto $\mathcal{N}_{a}\left(s, s^{\prime}\right)$ inclui todos os fatos novos com relação a fluentes inertes do domínio que são afetados por $a$ ). A condição (ii) vale se alguma sentença de efeito não-determinístico permite $p$ mudar (isso significa que o conjunto $\mathcal{N}_{a}\left(s, s^{\prime}\right)$ inclui todos os fatos relacionados a fluentes não-inertes do domínio que são possivelmente afetados por $a$ ). Note que a condição (ii) é necessária para garantir que o valor de $p$ seja considerado "novo" em $s^{\prime}$, mesmo quando seu valor nesse estado coincidir com aquele no estado $s$ (pois, nesse caso, a condição (i) não vale).

Agora, podemos definir a função de transição como a seguir.

Definição 3.3. $\mathcal{T}(s, a)$ é o conjunto de estados $s^{\prime} \in \mathcal{T}_{0}(s, a)$, para os quais $\mathcal{N}_{a}\left(s, s^{\prime}\right)$ é minimal com relação à inclusão de conjuntos, ou seja, para os quais não existe $s^{\prime \prime} \in \mathcal{T}_{0}(s, a)$, tal que $\mathcal{N}_{a}\left(s, s^{\prime \prime}\right)$ seja um subconjunto próprio de $\mathcal{N}_{a}\left(s, s^{\prime}\right)$.

A condição de minimalidade ${ }^{2}$ imposta pela Definição 3.3 é que garante a solução do problema de persistência temporal na semântica das linguagens $\mathcal{A R}$.

\subsection{Especificando um domínio em $\mathcal{A R}$}

Para tornar as definições da última seção mais claras, considere um ambiente de planejamento em que o agente é capaz de trocar uma lâmpada, além de ligar ou desligar um interruptor. Suponha que os estados desse ambiente sejam descritos pelos fluentes ligado, queimada e acesa; sendo que ligado é um fluente que indica o estado do interruptor e queimada e acesa são fluentes que indicam o estado da lâmpada. Usando a linguagem $\mathcal{A R}$ caracterizada por esses conjuntos de ações e fluentes, podemos descrever a dinâmica do ambiente de planejamento através da seguinte especificação de domínio:

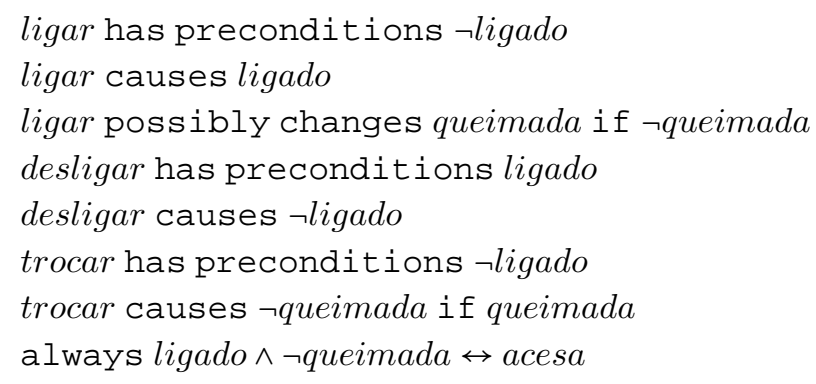

\footnotetext{
${ }^{2}$ Noção semelhante àquela de circunscrição, usada em lógica de predicados não-monotônica [Shanahan, 1997].
} 
Nessa especificação, o fluente ligado é um efeito direto da ação ligar; o fluente acesa é um efeito indireto (ou ramificado [Shanahan, 1997]) dessa mesma ação; e o fluente queimada é um fluente não-inerte, cujo valor é afetado de forma não-determinística pela execução da ação ligar.

Para simplificar a discussão sobre esse especificação, vamos usar $L$ e $\bar{L}$ para denotar as fórmulas atômicas (ligado is T) e (ligado is $\perp$ ), respectivamente. Analogamente, usaremos $Q$ e $\bar{Q}$ para o fluente queimada; e $A$ e $\bar{A}$ para o fluente acesa.

O Exemplo 3.2 mostra como o conjunto de estados de um domínio de planejamento pode ser obtido a partir de sua especificação em $\mathcal{A R}$.

Exemplo 3.2 (Conjunto de estados). Considere a especificação de domínio (3.7), onde são os fluentes que descrevem os estados do ambiente são ligado (L), queimada $(Q)$ e acesa $(A)$. Há oito valorações distintas para esses três fluentes:

$\{L Q A, L Q \bar{A}, L \bar{Q} A, L \bar{Q} \bar{A}, \bar{L} Q A, \bar{L} Q \bar{A}, \bar{L} \bar{Q} A, \bar{L} \bar{Q} \bar{A}\}$.

Entretanto, apenas quatro dessas oito valorações satisfazem a restrição imposta pela sentença (always ligado $\wedge$ queimada $\leftrightarrow$ acesa). Logo, de acordo com a Definição 3.2, o conjunto de estados do domínio especificado em (3.7) é:

$\{L Q \bar{A}, L \bar{Q} A, \bar{L} Q \bar{A}, \bar{L} \bar{Q} \bar{A}\}$.

O Exemplo 3.3 mostra como a função de transição de um domínio de planejamento pode ser obtida a partir de sua especificação em $\mathcal{A R}$.

Exemplo 3.3 (Transições). Considere a ação ligar, no domínio de planejamento especificado em (3.7), cuja precondição é $\neg$ ligado ( $\bar{L}$ ). Claramente, há apenas dois estados do domínio em que essa ação pode ser executada (aqueles estados em que o interruptor está desligado):

- Para o primeiro estado $(\bar{L} Q \bar{A})$, temos:

- $\mathcal{T}_{0}(\bar{L} Q \bar{A}$, ligar $)=\{L Q \bar{A}, L \bar{Q} A\}$

- $\mathcal{N}_{\text {ligar }}(\bar{L} Q \bar{A}, L Q \bar{A})=\{L\}$

- $\mathcal{N}_{\text {ligar }}(\bar{L} Q \bar{A}, L \bar{Q} A)=\{L, \bar{Q}, A\}$

Então, de acordo com a Definição 3.3, como o conjunto $\mathcal{N}_{\text {ligar }}(\bar{L} Q \bar{A}, L \bar{Q} A)$ não é minimal, segue que $\mathcal{T}(\bar{L} Q \bar{A}$, ligar $)=\{L Q \bar{A}\}$. De fato, a transição do estado $\bar{L} Q \bar{A}$ para o estado $L \bar{Q}$ A não faz mesmo sentido, já que a ação ligar não pode transformar uma lâmpada queimada em não-queimada.

- Para o segundo estado $(\bar{L} \bar{Q} \bar{A})$, temos. 
- $\mathcal{T}_{0}(\bar{L} \bar{Q} \bar{A}$, ligar $)=\{L Q \bar{A}, L \bar{Q} A\}$

- $\mathcal{N}_{\text {ligar }}(\bar{L} \bar{Q} \bar{A}, L Q \bar{A})=\{L, Q\}$

- $\mathcal{N}_{\text {ligar }}(\bar{L} \bar{Q} \bar{A}, L \bar{Q} A)=\{L, \bar{Q}, A\}$

Agora, os dois conjuntos $\mathcal{N}_{\text {ligar }}$ são minimais $(\{L, Q\} \not\{L, \bar{Q}, A\}$ e $\{L, \bar{Q}, A\} \not\{L, Q\})$. Logo, de acordo com a Definição 3.3, segue que $\mathcal{T}(\bar{L} \bar{Q} \bar{A}$, ligar $)=\{L Q \bar{A}, L \bar{Q} A\}$. De fato, quando a lâmpada não está queimada, a ação ligar pode queimá-la, já que esse é um efeito não-determinístico dessa ação.

As transições relativas às demais ações do domínio (3.7) podem ser obtidas de forma análoga.

O grafo de transições completo para o domínio especificado em (3.7), discutido nos Exemplos 3.2 e 3.3, pode ser visto na Figura 3.3.

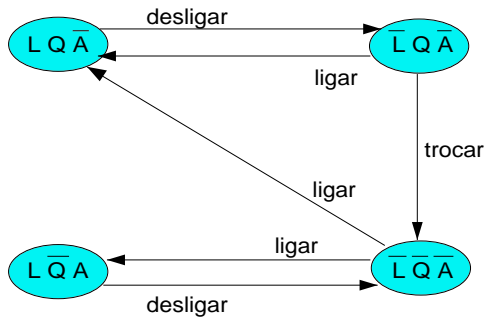

Figura 3.3: Grafo de transições para o domínio descrito em (3.7).

\subsubsection{Construção de modelos simbólicos}

Dada uma especificação de domínio $\mathcal{D}$ em $\mathcal{A R}$, podemos construir um modelo simbólico correspondente. O conjunto de estados $\mathcal{S}$ desse modelo pode ser codificado como segue:

$$
\mathcal{S} \doteq \bigwedge_{(\operatorname{always} \varphi) \in \mathcal{D}} \varphi
$$

Para codificar a função de transição do modelo simbólico, introduzimos uma variável act, sobre o conjunto de ações do domínio. Ademais, para representar os valores dos fluentes antes e após a execução de uma ação, introduzimos uma variável $p^{\prime}$ para cada fluente $p$ do domínio. A função de transição é representada por uma fórmula $\mathcal{T}$, definida sobre as variáveis $a c t, \vec{p}$ e $\vec{p}^{\prime}$. Atribuições 
a essas variáveis, satisfazendo $\mathcal{T}$, representam transições no modelo. Para obtermos $\mathcal{T}$, primeiro construímos a fórmula a seguir:

$$
\mathcal{T}_{0} \doteq \mathcal{S} \wedge \mathcal{S}\left[\vec{p}:=\vec{p}^{\prime}\right] \wedge \bigwedge_{\left(a \text { causes } \varphi^{\prime} \text { if } \varphi\right) \in \mathcal{D}}\left((a c t=a \wedge \varphi) \rightarrow \varphi^{\prime}\left[\vec{p}:=\vec{p}^{\prime}\right]\right)
$$

Intuitivamente, o conjunto $\mathcal{S}\left[\vec{p}:=\vec{p}^{\prime}\right]$ garante que as valorações para estados sucessores sejam estados do modelo; enquanto $\varphi^{\prime}\left[\vec{p}:=\vec{p}^{\prime}\right]$ impõe que os efeitos de uma ação $a$ (especificados por $\varphi^{\prime}$ ) devem valer nos estados sucessores, sempre que suas precondições (especificadas por $\varphi$ ) forem satisfeitas no estado corrente.

Note que a fórmula $\mathcal{T}_{0}$ identifica tudo o que deve mudar nos estados sucessores, como consequiência da execução de cada ação particular, mas não diz nada a respeito do que deve persistir. Podemos obter $\mathcal{T}$ (i.e., a relação de transição) minimizando as mudanças em $\mathcal{T}_{0}$, ou seja, eliminando todas as valorações onde variações nos fluentes são desnecessárias.

$\mathrm{Na}$ fórmula a seguir, assumimos que $p_{1}, \ldots, p_{m}\left(p_{m+1}, \ldots, p_{n}\right)$ são fluentes inertes (nãoinertes) de $\mathcal{D}$, listados de acordo com alguma enumeração fixa:

$$
\begin{aligned}
& \mathcal{T} \doteq \mathcal{T}_{0} \wedge \neg \exists v_{1} \ldots v_{n} .( \\
& \mathcal{T}_{0}\left[p_{1}^{\prime}:=v_{1}, \ldots, p_{n}^{\prime}:=v_{n}\right] \wedge \\
&\left(a \text { possibly changes } p_{j} \text { if } \varphi\right) \in \mathcal{D} \\
& \wedge\left((a c t=a \wedge \varphi) \rightarrow p_{j}^{\prime}=v_{j}\right) \wedge \\
&\left.\wedge\left(p_{i}=v_{i} \vee p_{i}^{\prime}=v_{i}\right) \wedge \widehat{i \in[1, \ldots, m]}^{\wedge}\left(p_{i}^{\prime} \neq v_{i}\right)\right)
\end{aligned}
$$

Intuitivamente, essa definição estabelece que, dada uma ação $a$, uma valoração para as variáveis $\vec{p}^{\prime}$ é compatível com uma valoração para as variáveis $\vec{p}$ se e só se satisfaz as condições de efeitos $\left(\mathcal{T}_{0}\right)$ e não existe outra valoração $\left(\neg \exists v_{1} \ldots v_{n}\right)$ que também satisfaça essas condições de efeitos $\left(\mathcal{T}_{0}\left[p_{1}^{\prime}:=v_{1}, \ldots, p_{n}^{\prime}:=v_{n}\right]\right)$, que seja compatível com a valoração em $\vec{p}$ com relação aos fluentes afetados por $\left((a c t=a \wedge \varphi) \rightarrow p_{j}^{\prime}=v_{j}\right)$ e que seja mais próxima do estado corrente (conjunção e disjunção iterativas no final da fórmula (3.10)).

A fórmula (3.10) pode ainda ser representada na forma de um diagrama de decisão binária ordenado e reduzido (ROBDD), como discutimos no último capítulo. Assim, daqui em diante, como os algoritmos para verificação de modelos podem operar diretamente sobre o modelo (simbólico) construído a partir de uma especificação de domínio em $\mathcal{A R}$, vamos supor que esse modelo de domínio (i.e., o grafo de transições) está disponível. 


\subsection{Sumário}

Nesse capítulo, mostramos que o arcabouço de verificação de modelos [Müller-Olm et al., 1999] pode ser adaptado para resolver problemas de planejamento automatizado [Ghallab et al., 2004]. Nesse contexto, o modelo considerado descreve a dinâmica do ambiente de planejamento, enquanto a propriedade a ser verificada descreve a meta do agente nesse ambiente.

Também apresentamos a definição formal de domínio de planejamento qualitativo e mostramos como um modelo explícito de domínio (i.e., grafo de transições) pode ser obtido a partir de uma descrição de ações numa linguagem de alto-nível chamada $\mathcal{A R}$ [Giunchiglia et al., 1997]. Finalmente, concluímos o capítulo mostrando o modelo obtido, apesar de explícito, pode ser representado eficientemente através do uso de ROBDDs [Bryant, 1992], cujos algoritmos de manipulação foram apresentados no Capítulo 2.

No próximo capítulo, discutiremos planejamento baseado em verificação de modelos para metas de alcançabilidade simples, em domínios de planejamento qualitativo descritos por meio de modelos explícitos. 


\section{Capítulo 4}

\section{Planejamento para metas de alcançabilidade simples}

\subsection{Metas de alcançabilidade simples}

Uma meta de alcançabilidade simples expressa uma condição a ser satisfeita no estado final do ambiente de planejamento, alcançado após a execução de um plano. Em planejamento clássico, onde o ambiente evolui de forma determinística, a noção de solução para um problema de planejamento para meta de alcançabilidade simples é clara. A execução de um plano nesse tipo de ambiente sempre produz uma única seqüência de estados possível e o estado final nessa seqüência deve satisfazer a meta de planejamento (i.e., deve ser um estado meta). Em planejamento sob incerteza, porém, a noção de solução é mais complexa. Como a execução de um mesmo plano num ambiente não-determinístico pode resultar em várias seqüências de estados distintas, uma solução para um problema de planejamento sob incerteza com meta de alcançabilidade simples deve ser caracterizada em termos de todas as seqüências de estados que essa solução pode gerar ao ser executada.

Nesse capítulo, definimos precisamente as três classes de soluções possíveis para um problema de planejamento sob incerteza qualitativa com meta de alcançabilidade simples; caracterizamos essas classes de soluções em termos da semântica de CTL; e, finalmente, descrevemos os algoritmos que garantem obter políticas em cada uma dessas três classes de soluções. 


\subsection{Problemas e soluções}

Nesse capítulo, adotamos a definição de domínio de planejamento qualitativo apresentada na Seção 3.2 (pág. 38).

Definição 4.1. Um problema de planejamento para meta de alcançabilidade simples é definido por uma tupla $\mathcal{P}=\left\langle\mathcal{D}, s_{0}, \phi\right\rangle$, onde:

- $\mathcal{D}=\langle\mathcal{S}, \mathcal{L}, \mathcal{T}\rangle$ é um domínio de planejamento qualitativo com assinatura $(\mathbb{P}, \mathbb{A})$;

- $s_{0} \in \mathcal{S}$ é o estado inicial do ambiente;

- $\phi$ é uma fórmula proposicional sobre $\mathbb{P}$.

Sejam $\mathcal{D}=\langle\mathcal{S}, \mathcal{L}, \mathcal{T}\rangle$ um domínio de planejamento com assinatura $(\mathbb{P}, \mathbb{A}), p \in \mathbb{P}$ uma proposição atômica e $\phi$ uma fórmula proposicional sobre $\mathbb{P}$. Definimos a relação de satisfatibilidade entre estados e fórmulas proposicionais como segue:

- $s \vDash p$ se só se $p \in \mathcal{L}(s)$;

- $s \vDash \neg \phi$ se e só se $s \not \neq$;

- $s \vDash \phi \wedge \phi^{\prime}$ se e só se $s \vDash \phi$ e $s \vDash \phi^{\prime}$;

- $s \vDash \phi \vee \phi^{\prime}$ se e só se $s \vDash \phi$ ou $s \vDash \phi^{\prime}$.

Assim, um problema de planejamento para metas de alcançabilidade simples $\mathcal{P}=\left\langle\mathcal{D}, s_{0}, \phi\right\rangle$ pode ser também definido pela tupla $\mathcal{P}=\left\langle\mathcal{D}, s_{0}, \mathcal{G}\right\rangle$, onde $\mathcal{G}$ é um conjunto de estados metas, ou seja, $\mathcal{G}=\{s \in \mathcal{S}: s \vDash \phi\}$.

Definição 4.2. Uma política $\pi$ para um domínio de planejamento qualitativo $\mathcal{D}$ é uma função parcial $\pi: \mathcal{S} \mapsto \mathbb{A}$, que mapeia estados em ações; tal que, para todo estado $s \in \mathcal{S}$, se $\pi$ está definida para s, então $\pi(s) \in\{a \in \mathbb{A}: \mathcal{T}(s, a) \neq \varnothing\}$.

Dada uma política $\pi$ para um domínio $\mathcal{D}$, o conjunto de estados alcançáveis por $\pi$, denotado por $\mathcal{S}_{\pi}$, é $\{s:(s, a) \in \pi\} \cup\left\{s^{\prime}:(s, a) \in \pi\right.$ e $\left.s^{\prime} \in \mathcal{T}(s, a)\right\}$. A estrutura de execução de $\pi$ (ou grafo de transições induzido por $\pi$ ), denotada por $\mathcal{D}_{\pi}$, é o subgrafo de $\mathcal{D}$ que tem $\mathcal{S}_{\pi}$ como conjunto de estados e que contém todas as transições induzidas pelas ações em $\pi$. Por exemplo, a estrutura de execução da política $\pi=\left\{\left(s_{0}, a_{01}\right),\left(s_{1}, a_{15}\right),\left(s_{2}, a_{20}\right)\right\}$, para o domínio de planejamento na Figura 4.1-a, pode ser vista na Figura 4.1-b: 


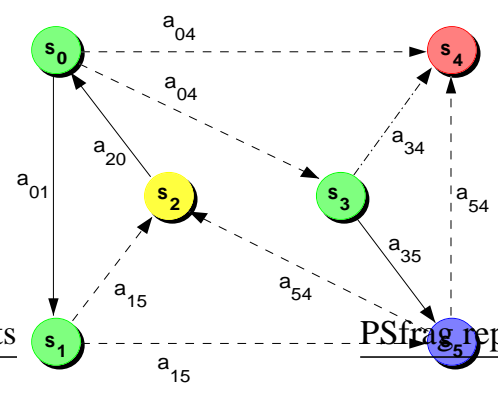

(a) domínio

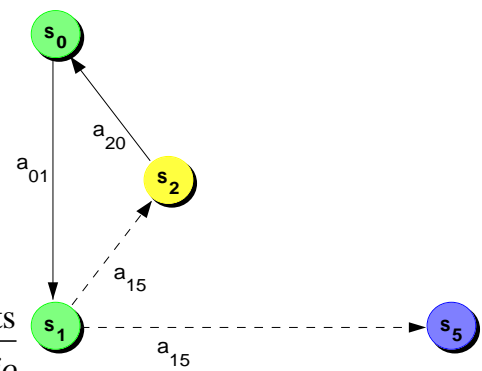

(b) estrutura de execução

Figura 4.1: Domínio e estrutura de execução da política $\pi=\left\{\left(s_{0}, a_{01}\right),\left(s_{1}, a_{15}\right),\left(s_{2}, a_{20}\right)\right\}$.

Informalmente, uma solução (ou plano) para um problema de planejamento qualitativo $\mathcal{P}$ é uma política, definindo um comportamento condicional e iterativo, que pode levar o agente do estado inicial $s_{0}$ até um estado meta $s_{n} \in \mathcal{G}$.

Exemplo 4.1 (Solução). Considere o domínio de planejamento na Figura 4.1-a. Nesse domínio, cada estado corresponde a uma localização no ambiente de planejamento e o agente nesse ambiente é um robô que se move de uma localização para outra. Suponha que o agente esteja inicialmente no estado $s_{0}$ e que sua meta seja alcançar o estado $s_{5}$. Então, a política $\pi=\left\{\left(s_{0}, a_{01}\right),\left(s_{1}, a_{15}\right),\left(s_{2}, a_{20}\right)\right\}$ é uma solução para esse problema de planejamento. $O$ comportamento condicional definido por $\pi$ deve-se ao fato da escolha de ações depender do estado corrente do ambiente (e.g., se estiver em $s_{0}$, execute $a_{01}$ ) e o comportamento iterativo, ao fato do agente poder visitar uma mesma localização um número arbitrário de vezes (e.g., executando a ação $a_{15}$ no estado $s_{1}$, o agente pode retornar ao estado inicial $\left.s_{0}\right)$.

Soluções para problemas de planejamento para metas de alcançabilidade simples podem ser classificadas em três diferentes classes, de acordo com a sua qualidade: uma solução fraca é uma política que pode atingir um estado meta mas que, devido ao não-determinismo, não oferece nenhuma garantia disso [Cimatti et al., 1997]; uma solução forte é uma política que sempre alcança um estado meta, independentemente do não-determinismo [Cimatti et al., 1998]; e uma solução forte-cíclica é uma política que sempre alcança um estado meta, sob a hipótese de que sua execução eventualmente conseguirá sair de todos os ciclos existentes [Daniele et al., 1999].

Exemplo 4.2 (Diferentes qualidades de soluções). Considere as políticas a seguir, levando em conta o domínio na Figura 4.1-a: 
- $\pi_{1}=\left\{\left(s_{0}, a_{04}\right),\left(s_{3}, a_{35}\right),\left(s_{5}, a_{54}\right)\right\}$

- $\pi_{2}=\left\{\left(s_{0}, a_{04}\right),\left(s_{3}, a_{34}\right)\right\}$

- $\pi_{3}=\left\{\left(s_{0}, a_{01}\right),\left(s_{1}, a_{15}\right),\left(s_{2}, a_{20}\right),\left(s_{5}, a_{54}\right)\right\}$

Essas políticas representam diferentes estratégias que o agente pode empregar para alcançar o estado meta $s_{4}$, partindo do estado inicial $s_{0}$. A política $\pi_{1}$ é uma solução fraca: se o agente atinge o estado $s_{2}$ (via ação $a_{54}$ ), ele não consegue mais alcançar sua meta. A política $\pi_{2}$ é uma solução forte: independentemente da incerteza existente com relação aos efeitos da ação $a_{04}$, o agente sempre atinge a sua meta (seja diretamente a partir do estado $s_{0}$, ou indiretamente a partir do estado $\left.s_{3}\right)$. Finalmente, $\pi_{3}$ é uma solução forte-cíclica. Note que essa última política descreve um comportamento iterativo, já que o agente pode ter que repetir várias vezes uma mesma ação (e.g., $\left.a_{20}\right)$ antes de atingir sua meta. Enquanto as políticas $\pi_{1}$ e $\pi_{2}$, garantidamente, atingem um estado terminal (não necessariamente meta), a política $\pi_{3}$ pode ficar executando "infinitamente".

\subsubsection{Caracterização das classes de soluções em CTL}

Um ponto importante a observar é que, de acordo com a Definição 4.1, um problema de planejamento qualitativo $\mathcal{P}=\left\langle\mathcal{D}, s_{0}, \phi\right\rangle$ define apenas a meta de planejamento (especificada pela fórmula proposicional $\phi$ ). A qualidade da política particular que deverá ser sintetizada como solução para o problema $\mathcal{P}$, entretanto, é uma decisão que depende do agente (ou seja, do método de planejamento empregado). Uma questão que surge, então, é a seguinte:

Como podemos especificar metas de alcançabilidade simples, indicando também a qualidade da solução desejada?

Como a qualidade de uma política (solução) depende de que caminhos de execução ela é capaz de gerar, a lógica CTL torna-se uma escolha natural para especificar metas de alcançabilidade simples, com a qualidade de solução desejada.

Seja $\pi$ uma política com estrutura de execução $\mathcal{D}_{\pi}$. Abstraindo-se os rótulos nas transições em $\mathcal{D}_{\pi}$, obtemos uma estrutura de Kripke correspondente, denotada por $\mathcal{K}\left(\mathcal{D}_{\pi}\right)$. Com base nessa estrutura de Kripke, podemos caracterizar as classes de soluções conforme a seguir. 
Definição 4.3. Seja $\pi$ (com estrutura de execução $\mathcal{D}_{\pi}$ ) uma política para um problema de planejamento qualitativo $\mathcal{P}=\left\langle\mathcal{D}, s_{0}, \phi\right\rangle$, onde $\phi$ é uma fórmula proposicional sobre $\mathbb{P}$. Dizemos que $\pi$ é uma solução:

- fraca para $\mathcal{P}$ se e só se $\left(\mathcal{K}\left(\mathcal{D}_{\pi}\right), s_{0}\right) \vDash \exists \diamond \phi$;

- forte para $\mathcal{P}$ se e só se $\left(\mathcal{K}\left(\mathcal{D}_{\pi}\right), s_{0}\right) \vDash \forall \diamond \phi$;

- forte-cíclica para $\mathcal{P}$ se e só se $\left(\mathcal{K}\left(\mathcal{D}_{\pi}\right), s_{0}\right) \vDash \forall \square \exists \diamond \phi$.

Intuitivamente uma política $\pi$ é uma solução fraca para $\mathcal{P}$, se algum caminho a partir de $s_{0}$, em $\mathcal{K}\left(\mathcal{D}_{\pi}\right)$, alcança um estado final satisfazendo $\phi$; é uma solução forte, se todo caminho a partir de $s_{0}$, em $\mathcal{K}\left(\mathcal{D}_{\pi}\right)$, é finito e alcança um estado final satisfazendo $\phi$; e é uma solução forte-cíclica, se todo caminho a partir de $s_{0}$, em $\mathcal{K}\left(\mathcal{D}_{\pi}\right)$, alcança um estado final satisfazendo $\phi$.

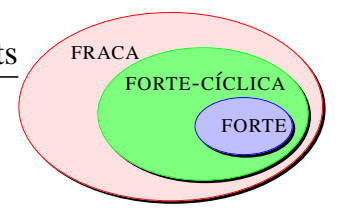

Figura 4.2: Classes de soluções em planejamento qualitativo.

Como podemos ver na Figura 4.2, toda solução forte é também forte-cíclica (pois todo modelo temporal para $\forall \diamond \phi$ é também um modelo para $\forall \square \exists \diamond \phi$ ) e toda solução forte-cíclica é também fraca (pois todo modelo para $\forall \square \exists \diamond \phi$ é também um modelo para $\exists \diamond \phi$ ). Soluções estritamente fracas e soluções fortes correspondem a dois casos extremos de satisfação de metas de alcançabilidade simples. Intuitivamente, soluções estritamente fracas correspondem a "planos otimistas", enquanto soluções fortes correspondem a "planos seguros".

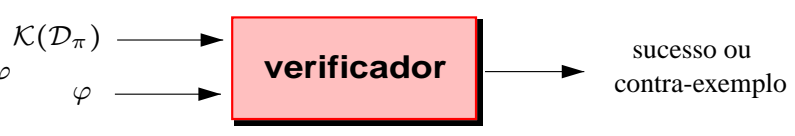

Figura 4.3: Validação de política com relação a uma meta de planejamento.

É importante notar que a formalização das classes de soluções para metas de alcançabilidade simples com qualidade embutida, proposta nessa tese (Definição 4.3), evidencia o fato de que um verificador de modelos para CTL pode ser empregado diretamente para validar políticas dadas (Figura 4.3), mas não para sintetizá-las a partir da especificação do domínio de planejamento. 
De fato, algoritmos para síntese de políticas precisam levar em conta os diferentes tipos de transições (causadas pelas diferentes ações do domínio) e, portanto, não podem ser baseados apenas na semântica de CTL (como faz um verificador de modelos). Isto é, para decidir que ações que irão compor as políticas, os algoritmos de síntese necessitam de um tipo de controle que não pode ser justificado diretamente pela semântica de CTL.

\subsection{Algoritmos para síntese de políticas}

A seguir, apresentamos os algoritmos capazes de obter soluções com diferentes qualidades, para problemas de planejamento qualitativo com metas de alcançabilidade simples.

\subsubsection{Planejamento forte}

O algoritmo de planejamento FORTE [Cimatti et al., 1998] recebe como entrada um problema de planejamento qualitativo para meta de alcançabilidade simples $\mathcal{P}=\left\langle\mathcal{D}, s_{0}, \phi\right\rangle$ e devolve uma solução forte (se existir) ou fracasso. Para encontrar uma solução forte, esse algoritmo realiza uma busca regressiva em largura, partindo do conjunto de estados metas $\mathcal{G}=\{s \in \mathcal{S}: s \vDash \phi\}$, até alcançar o estado inicial $s_{0}$, através da aplicação iterativa da função PrÉImAGEMForte ao conjunto corrente de estados atingíveis $S^{\prime}$, definida como:

$$
\operatorname{PrÉ} \text { IMAGEMForte }\left(S^{\prime}\right)=\left\{(s, a): s \in \mathcal{S}, a \in \mathbb{A} \text { e } \varnothing \neq \mathcal{T}(s, a) \subseteq S^{\prime}\right\}
$$

Note que essa função resulta num conjunto de estados ${ }^{1}$ cujos sucessores estão todos no conjunto $S^{\prime}$. Como a regressão inicia com $S^{\prime}=\mathcal{G}$, podemos garantir que todo caminho iniciando num estado no conjunto $\operatorname{Pré}$ Imagemforte $\left(S^{\prime}\right)$ leva, necessariamente, a um estado meta em $\mathcal{G}$; ademais, como cada novo estado em $\operatorname{PrÉ}$ ImagemForte $\left(S^{\prime}\right)$ só tem sucessores em $S^{\prime}$, não haverá ciclos nesses caminhos. Portanto, podemos garantir que o algoritmo ForTe termina em um dos casos: (i) quando o estado inicial estiver no conjunto $S^{\prime}$ (nesse caso, $\pi_{i}$ é uma solução forte para $\mathcal{P}$ ); ou (ii) quando um ponto-fixo mínimo, a partir do qual o conjunto $S^{\prime}$ não pode mais ser estendido, for atingido (nesse caso, não existe solução forte para $\mathcal{P}$ ).

\footnotetext{
${ }^{1}$ Na verdade PRÉIMAGEMForTE resulta numa política, ou seja, um conjunto de pares $(s, a)$, mas estamos nos referindo apenas aos estados mapeados por essa política.
} 


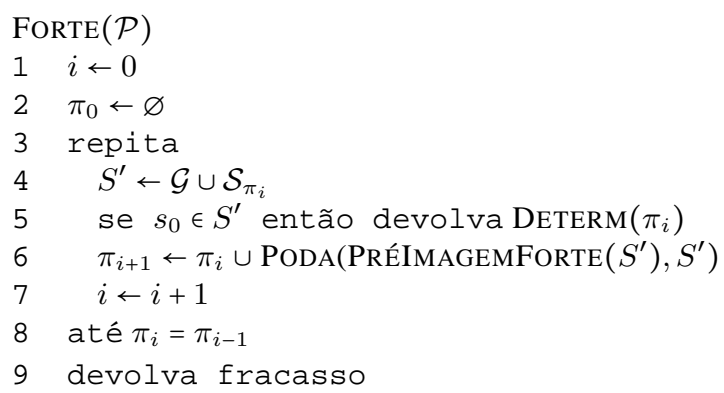

Para eliminar da pré-imagem forte de $S^{\prime}$ os estados que já estavam em $S^{\prime}$ (e que, portanto, já foram mapeados a alguma ação em uma das iterações anteriores), o algoritmo de planejamento FORTE utiliza a função PODA, definida como:

$$
\operatorname{PodA}\left(\pi, S^{\prime}\right)=\left\{(s, a) \in \pi: s \notin S^{\prime}\right\}
$$

Essa função tem duas finalidades importantes: (i) garantir que a política sintetizada seja acíclica e (ii) garantir que a política sintetizada seja ótima no pior caso (veja o Exemplo 4.3).

Finalmente, para garantir que a política devolvida seja determinística, i.e., que associe no máximo uma ação de $\mathbb{A}$ a cada estado de $\mathcal{S}$, o algoritmo Forte utiliza a função $\operatorname{Determ}(\pi)$, que devolve uma política $\pi^{\prime} \subseteq \pi$ tal que $\mathcal{S}_{\pi^{\prime}}=\mathcal{S}_{\pi}$ e $\pi^{\prime}$ satisfaz a condição de que para cada estado $s \in \mathcal{S}_{\pi^{\prime}}$ exista uma única ação associada.

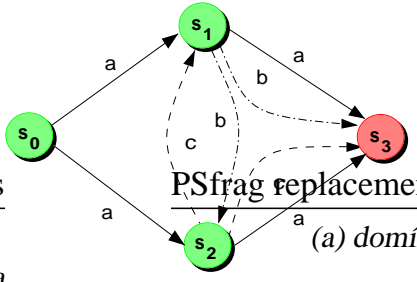

(a) domínio

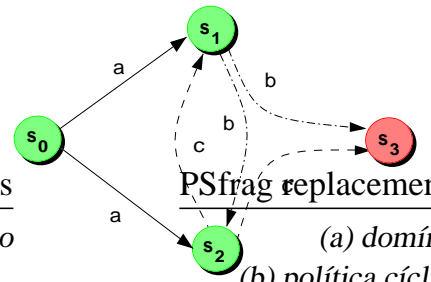

(b) política cíclica

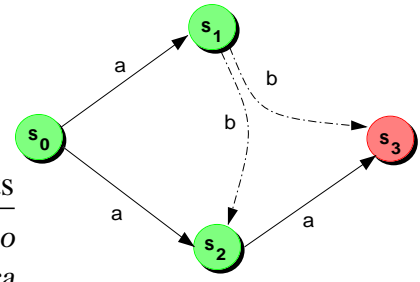

(c) política sub-ótima

Figura 4.4: Finalidade da função PoDA.

Exemplo 4.3 (Necessidade da poda). Considere o domínio na Figura 4.4-a. Calculando a préimagem forte do conjunto $S^{\prime}=\left\{s_{3}\right\}$, obtemos a política parcial $\pi_{1}=\left\{\left(s_{1}, a\right),\left(s_{2}, a\right)\right\}$. Note que, nesse momento, as ações b e c são descartadas; pois elas levam a estados que estão fora do conjunto $S^{\prime}$. Na segunda iteração, o conjunto $S^{\prime}$ é atualizado para $\left\{s_{1}, s_{2}, s_{3}\right\}$. Então, calculando a pré-imagem forte de $S^{\prime}$, obtemos $\pi_{2}=\left\{\left(s_{1}, a\right),\left(s_{1}, b\right),\left(s_{2}, a\right),\left(s_{2}, c\right)\right\}$. Portanto, 
sem podar os novos pares $\left(s_{1}, b\right)$ e $\left(s_{2}, c\right)$ em $\pi_{2}$, que mapeiam estados que já foram mapeados na primeira iteração, a função Determ pode devolver como solução final a política da Figura 4.4-b, que é cíclica; ou então a política da Figura 4.4-c, que é sub-ótima no pior caso.

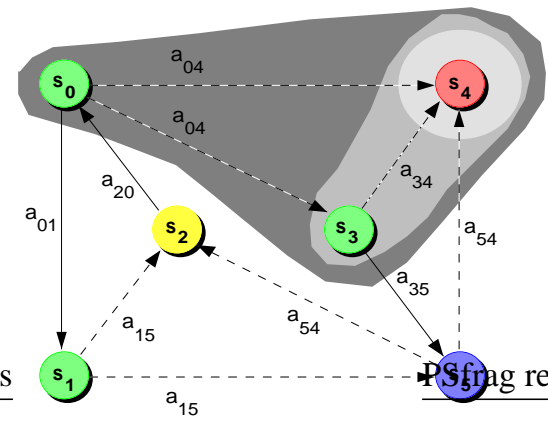

(a) regressão

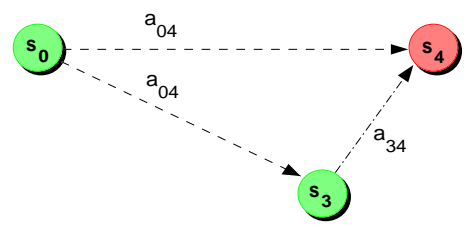

(b) estrutura da política sintetizada

Figura 4.5: Funcionamento do algoritmo ForTe, para alcançar $s_{4}$, a partir de $s_{0}$.

Exemplo 4.4 (Funcionamento do algoritmo FORTE). Considere o domínio de planejamento na Figura 4.5-a e suponha que o problema de planejamento nesse domínio seja alcançar o estado meta $s_{4}$, a partir do estado inicial $s_{0}$. O funcionamento do algoritmo FORTE, ao resolver esse problema, é descrito a seguir:

- Na primeira iteração, temos:

$S^{\prime}=\left\{s_{4}\right\}$

$\operatorname{PrÉ}$ IMAgemForte $\left(S^{\prime}\right)=\left\{\left(s_{3}, a_{34}\right)\right\}$

$\operatorname{Poda}\left(\operatorname{PrÉ}\right.$ ImagemForte $\left.\left(S^{\prime}\right), S^{\prime}\right)=\left\{\left(s_{3}, a_{34}\right)\right\}$

$\pi_{1}=\left\{\left(s_{3}, a_{34}\right)\right\}$

Note que, nessa iteração, a função de pré-imagem forte não seleciona o par $\left(s_{0}, a_{04}\right)$; pois, apesar da execução da ação $a_{04}$ no estado $s_{0}$ levar ao estado $s_{4} \in S^{\prime}$, ela também pode levar ao estado $s_{3}$, que não está em $S^{\prime}$. Ademais, como nenhum estado foi mapeado em uma iteração anterior, a função de poda não tem efeito.

- Na segunda iteração, temos:

$$
\begin{aligned}
& \mathcal{S}^{\prime}=\left\{s_{3}, s_{4}\right\} \\
& \text { PréImagemForte }\left(S^{\prime}\right)=\left\{\left(s_{0}, a_{04}\right),\left(s_{3}, a_{34}\right)\right\} \\
& \text { Poda(PréImagemForte } \left.\left(S^{\prime}\right), S^{\prime}\right)=\left\{\left(s_{0}, a_{04}\right)\right\} \\
& \pi_{2}=\left\{\left(s_{0}, a_{04}\right),\left(s_{3}, a_{34}\right)\right\}
\end{aligned}
$$


Nessa iteração, a função de pré-imagem forte não seleciona o par $\left(s_{5}, a_{54}\right)$; pois a execução da ação $a_{54}$ no estado $s_{5}$ pode levar também ao estado $s_{2}$, que não está em $S^{\prime}$. Ademais, como o estado $s_{3}$ já foi mapeado na primeira iteração, a função de poda descarta o par $\left(s_{3}, a_{34}\right)$. É claro que, para esse exemplo, não é necessário descartar esse par; porém, se o estado $s_{3}$ tivesse sido mapeado a uma nova ação (diferente de $a_{34}$ ), isso seria imprescindivel (vide Exemplo 4.3).

- Finalmente, como na terceira iteração a condição $s_{0} \in\left(\mathcal{G} \cup \mathcal{S}_{\pi_{2}}\right)$ é satisfeita, o algoritmo FORTE devolve $\pi_{2}$ (Figura 4.5-b) como resposta.

\subsubsection{Planejamento fraco}

O algoritmo de planejamento FrACo [Cimatti et al., 1997] é muito semelhante ao algoritmo de planejamento FORTE, mas usa uma função de pré-imagem fraca:

$$
\operatorname{PrÉ} \text { ImagemFraca }\left(S^{\prime}\right)=\left\{(s, a): s \in \mathcal{S}, a \in \mathbb{A} \text { e } \mathcal{T}(s, a) \cap S^{\prime} \neq \varnothing\right\}
$$

Essa função resulta num conjunto de estados $S$ a partir dos quais é possível atingir estados em $S^{\prime}$; porém, não há garantia de que todas as transições realizadas a partir de estados em $S$ realmente levem a estados em $S^{\prime}$. É por esse motivo que estados terminais que não são estados metas (e.g., estados $s_{2}$ e $s_{4}$ na Figura 4.6-b) podem surgir na estrutura de execução das políticas devolvidas como solução pelo algoritmo FraCo.

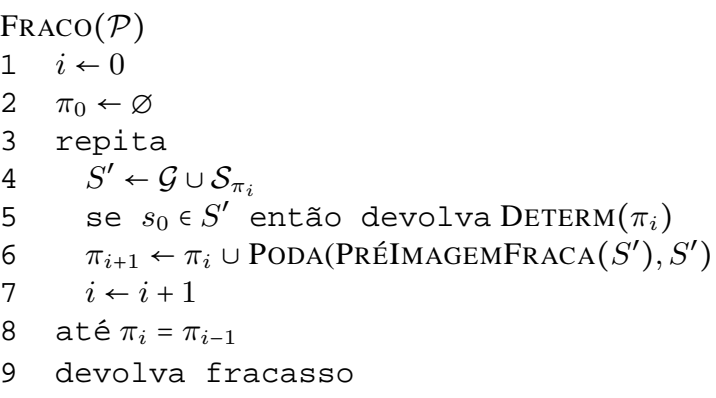

\subsubsection{Planejamento forte-cíclico}

O algoritmo de planejamento ForTECíclico [Daniele et al., 1999] inicia com uma política universal $\pi_{0}=\{(s, a): s \in \mathcal{S}, a \in \mathbb{A} \mathrm{e} \mathcal{T}(s, a) \neq \varnothing\}$, contendo todos os pares estado-ação possíveis 


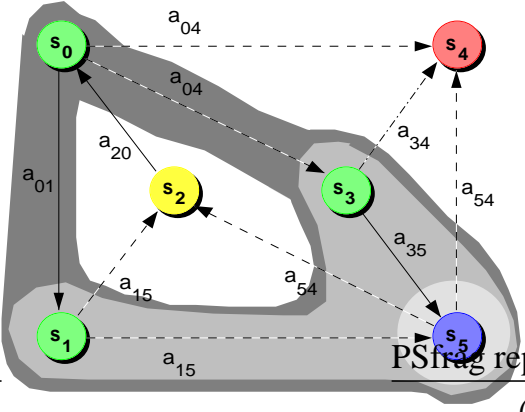

(a) regressão

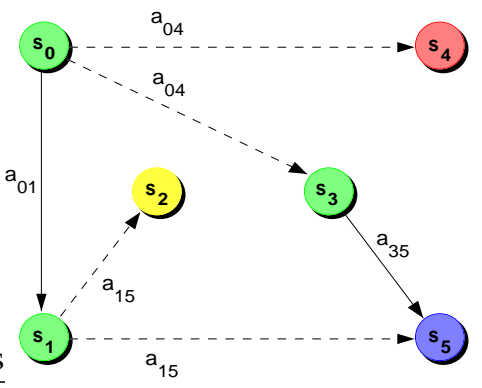

(b) estrutura

Figura 4.6: Funcionamento do algoritmo FRACO, para alcançar $s_{5}$, a partir de $s_{0}$.

no domínio considerado. Em seguida, iterativamente, vai eliminando os pares que levam a estados fora dessa política. A eliminação é implementada pelas funções PodABECo, que remove todos os pares $(s, a) \in \pi_{i}$, a partir dos quais é impossível atingir um estado meta; e PoDAForA, que remove todos os pares $(s, a) \in \pi_{i}$ que levam a estados fora de $\mathcal{S} \cup \mathcal{S}_{\pi_{i}}$. Como PodaFora pode causar o surgimento de novos becos e PODABECo pode causar o surgimento de novas transições para fora do conjunto $\mathcal{G} \cup \mathcal{S}_{\pi_{i+1}}$, o algoritmo prossegue até que a convergência seja atingida. Nesse ponto, caso a política obtida não cubra o estado inicial $s_{0}$, o algoritmo devolve fracasso; caso contrário, as transições que geram ciclos não-triviais são removidas por PODARETROCESSO e a política determinística resultante é devolvida como solução.

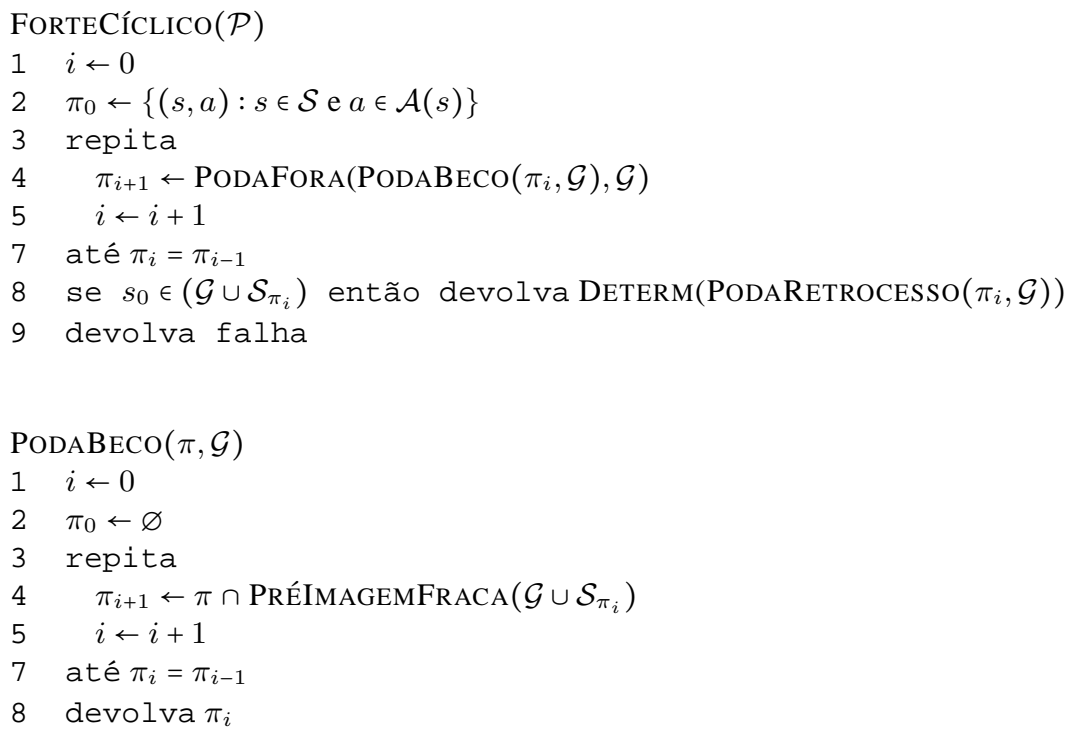




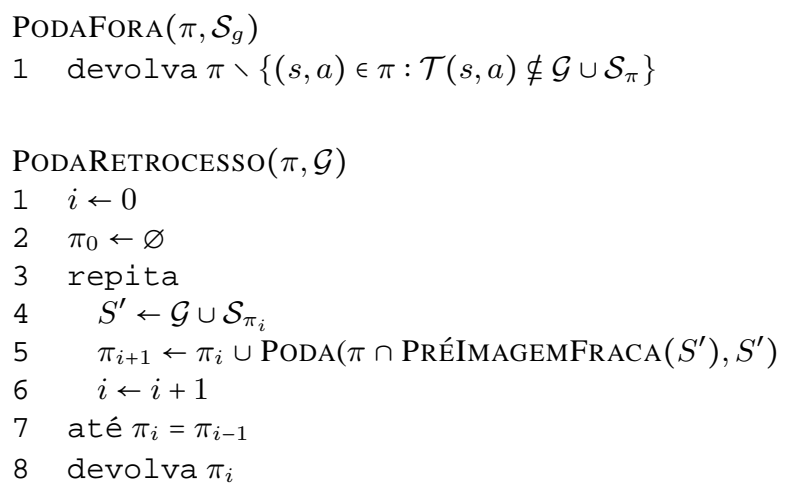

Exemplo 4.5 (Funcionamento do algoritmo ForTeCíclico). Na Figura 4.7, podemos acompanhar o funcionamento do algoritmo FORTECICLICO, ao tentar alcançar o estado meta $s_{5}$, a partir do estado inicial $s_{0}$. Primeiro o beco $s_{4}$, para o qual não há ação executável, é removido. Em seguida, todas as transições que levam para "fora" são eliminadas. Como esse é um problema muito simples, o algoritmo termina na primeira iteração. Em problemas mais complexos, a eliminação de transições poderia causar o surgimento de novos becos e mais iterações seriam necessárias para que uma solução fosse obtida.

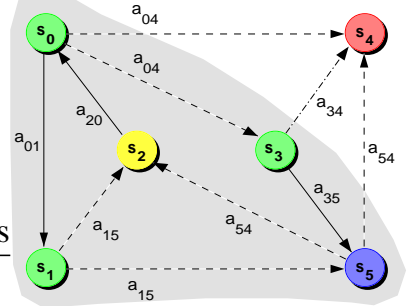

(a) política inicial

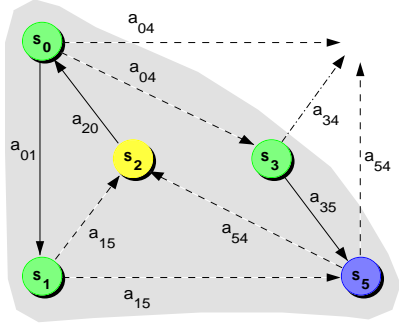

(b) poda beco

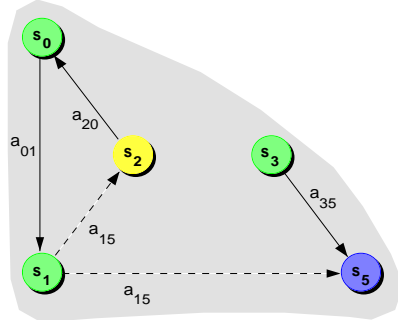

(c) poda fora

Figura 4.7: Funcionamento do algoritmo ForTECíclico, para alcançar $s_{5}$, a partir de $s_{0}$.

\subsection{Sumário}

Nesse capítulo, definimos o problema de planejamento qualitativo para metas de alcançabilidade simples [Giunchiglia \& Traverso, 1999, Ghallab et al., 2004] e mostramos que uma solução para esse tipo de problema pode ter diferentes qualidades, dependendo dos caminhos de execução que ela é capaz de produzir no domínio de planejamento qualitativo considerado. 
Em seguida, caracterizamos as classes de soluções e mostramos que, usando a lógica temporal de tempo ramificado CTL [Clarke \& Emerson, 1982], podemos especificar metas de alcançabilidade simples, assim como a qualidade da soluções desejadas. Também mostramos que, quando metas de alcançabilidade simples são especificadas em CTL, o arcabouço de verificação de modelos pode ser usado diretamente apenas para validar políticas, mas não para sintetizá-las.

Finalmente, concluímos o capítulo apresentando algoritmos de planejamento capazes de sintetizar políticas em cada uma das classes de soluções [Cimatti et al., 1997, Cimatti et al., 1998, Daniele et al., 1999]. Esses algoritmos, apesar de utilizarem técnicas de verificação de modelos, precisam também implementar um tipo de controle para escolhas de ações que não pode ser justificado diretamente com base em CTL; pois a semântica dessa lógica não é capaz de distinguir os diferentes tipos de transições que são causadas pelas diferentes ações existentes no domínio de planejamento.

No próximo capítulo, mostramos como podemos estender a expressividade das metas de alcançabilidade simples (herdadas do planejamento clássico) e apresentamos os algoritmos necessários para tratar uma classe de metas mais expressiva, que denominamos metas de alcançabilidade estendidas. Para tanto, propomos uma nova lógica ( $\alpha$-CTL) cuja semântica é capaz de justificar o tipo de controle de escolha de ações que precisamos em algoritmos de planejamento. 


\section{Capítulo 5}

\section{Planejamento para metas de alcançabilidade estendidas}

\subsection{Metas estendidas}

Uma meta estendida é qualquer especificação de meta que estende, de alguma forma, a expressividade de uma meta de alcançabilidade simples. Metas estendidas constituem uma classe bastante abrangente de metas que inclui, por exemplo, metas que especificam algum tipo de controle de busca [Kabanza et al., 1997], metas que especificam comportamentos de programas de controle [Ramadge \& Wonham, 1989, Ziller \& Schneider, 2005], metas que especificam qualidades mistas de solução [Baral \& Zhao, 2006] e metas que especificam uma ordem de preferência entre submetas [Krulwich, 1992]. Ao contrário de metas de alcançabilidade simples, que expressam condições a serem satisfeitas apenas no estado final alcançado pela execução da política, metas estendidas podem expressar condições que devem ser satisfeitas ao longo de toda execução da política [Bacchus \& Kabanza, 1996, Pistore \& Traverso, 2001, Baral \& Zhao, 2006].

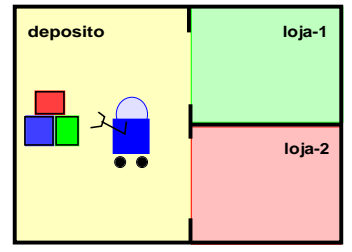

Figura 5.1: Cenário para o agente "entregador".

Exemplo 5.1 (Meta estendida). Considere a situação ilustrada na Figura 5.1 e suponha que a 
60CAPÍTULO 5. PLANEJAMENTO PARA METAS DE ALCANÇABILIDADE ESTENDIDAS

meta do agente seja "manter-se entregando caixas nas lojas 1 e 2, alternadamente". Essa meta é bastante diferente daquelas consideradas no planejamento clássico; particularmente, uma política que a satisfaça nunca alcança um estado final. Também é fácil perceber que, para satisfazê-la, uma política precisa associar duas ações distintas ao estado depósito: entregar-na-loja-1 e entregar-na-loja-2. Conseqüentemente, também precisaríamos manter alguma informação adicional que permitisse ao agente escolher a ação correta em cada momento (pois, havendo duas ações associadas ao mesmo estado, a escolha do agente não dependeria mais apenas do estado corrente do ambiente de planejamento).

O Exemplo 5.1 mostra que, no caso geral, metas estendidas não admitem políticas da forma $\pi: \mathcal{S} \mapsto \mathcal{A}$ como solução (note que para satisfazer a meta considerada no Exemplo 5.1, o mapeamento realizado pela política não pode ser feito em função apenas do estado corrente). Portanto, além do estado corrente, políticas para metas estendidas também podem precisar levar em conta o contexto corrente de execução, ou seja, o estado interno do agente no momento em que uma ação é selecionada para execução.

Política estendida. Quando a informação adicional necessária para a seleção de ação é limitada, a solução para uma meta estendida pode ser representada por uma política estendida $\hat{\pi}$ : $\mathcal{S} \times \mathcal{C} \mapsto \mathbb{A}$, onde $\mathcal{C} \neq \varnothing$ é um conjunto finito de contextos de execução [Ghallab et al., 2004]. Assim, dada uma política estendida $\hat{\pi}$, uma transição induzida por $\hat{\pi}$ é uma tupla da forma $(s, c) \stackrel{a}{\rightarrow}\left(s^{\prime}, c^{\prime}\right)$, onde $s, s^{\prime} \in \mathcal{S}, c, c^{\prime} \in \mathcal{C}$ e $a \in \mathbb{A}$; e um caminho na estrutura de execução dessa política é uma sequiência de transições da forma $\left(s_{0}, c_{0}\right) \stackrel{a_{0}}{\rightarrow}\left(s_{1}, c_{1}\right) \stackrel{a_{1}}{\rightarrow}\left(s_{2}, c_{2}\right) \stackrel{a_{2}}{\rightarrow}\left(s_{3}, c_{3}\right) \ldots$ Por exemplo, a estrutura de execução de uma política estendida que soluciona o problema de planejamento considerado no Exemplo 5.1 pode ser vista na Figura 5.2. Note que, nessa política, os contextos de execução voltou-da-loja-1 e voltou-da-loja-2 não são proposições de descrição de estados e, portanto, não podem ser obtidos pela simples observação do estado corrente do ambiente de planejamento.

Apesar de uma política estendida ser uma função parcial sobre $\mathcal{S} \times \mathcal{C}$, ainda assim, o espaço de busca para metas estendidas pode aumentar consideravelmente; no pior caso, o tamanho do espaço de estados do domínio de planejamento é multiplicado pela cardinalidade do conjunto de contextos, ou seja, o tamanho do espaço de busca é $O(|\mathcal{S}| \times|\mathcal{C}|)$. 


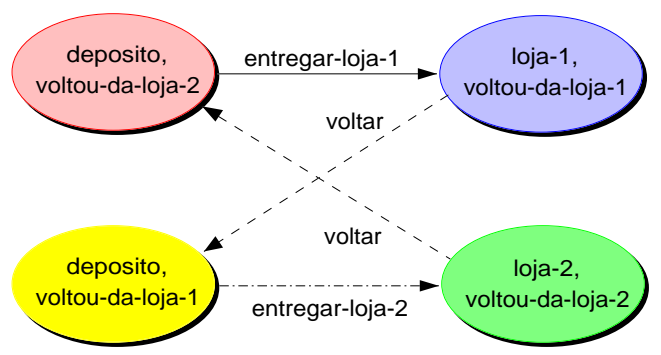

Figura 5.2: Estrutura de execução da política estendida para o Exemplo 5.1.

Linguagens extra-lógicas. A especificação de metas estendidas também pode requerer o uso de linguagens extra-lógicas especializadas (e.g., EAGLE [Lago et al., 2002]). Considere, por exemplo, a meta de planejamento: "tente alcançar p; se falhar, alcance q". Não há fórmula CTL que seja capaz de expressar essa meta [Ghallab et al., 2004]. Uma política que satisfaça a fórmula $\exists \diamond p$ (tente alcançar $p$ ) não garante que o agente fará o possível para alcançar $p$, mas apenas que ele terá uma chance de alcançar $p$. Além disso, não há como expressar o significado de "se falhar". Uma fórmula que tentasse expressar essa idéia usando disjunção (e.g., $\exists \diamond p \vee \exists \diamond q$ ou $\neg \exists \diamond p \rightarrow \exists \diamond q$ ) não poderia impedir que o planejador encontrasse uma solução que ignorasse completamente a primeira parte da meta $(\exists \diamond p)$, satisfazendo apenas a segunda $(\exists \diamond q)$.

\subsubsection{Metas de alcançabilidade estendidas}

Metas de alcançabilidade estendidas (que incluem metas de alcançabilidade simples como caso especial) constituem uma subclasse da classe de metas estendidas. Uma meta de alcançabilidade estendida, além de especificar uma condição a ser alcançada ao final da execução de uma política, também estabelece uma condição a ser preservada (ou evitada) em todos os estados visitados durante a execução dessa política.

Definição 5.1. Uma meta de alcançabilidade estendida é um par de fórmulas $\left(\varphi_{1}, \varphi_{2}\right)$, onde $\varphi_{1}$ especifica uma condição a ser preservada durante a execução de uma política e $\varphi_{2}$ é uma condição a ser alcançada ao final da execução dessa política.

Exemplo 5.2 (Meta de alcançabilidade estendida). Considere um problema em que a meta de um robô móvel é deslocar-se até um certo local de seu ambiente. Evidentemente, qualquer política de comportamento que leve o robô ao local desejado é uma solução para esse problema. 
Entretanto, suponha que no ambiente existam locais de risco para o robô. Nesse caso, além de alcançar o local desejado, seria interessante que o robô não fosse danificado durante o seu trajeto. Assim, uma solução ideal para esse problema seria uma política que garantisse ao robô alcançar o local desejado, sempre evitando locais de risco (condição que só pode ser especificada por meio de uma meta de alcançabilidade estendida).

Claramente, metas de alcançabilidade estendidas são menos expressivas que metas estendidas; entretanto, elas são mais expressivas que as metas de alcançabilidade simples (consideradas na maioria dos planejadores descritos na literatura), pois permitem estabelecer preferências entre os possíveis estados intermediários visitados durante a execução de uma política. Além disso, metas de alcançabilidade estendidas permitem uma maior eficiência de planejamento, quando comparadas às metas estendidas mais gerais, e ainda podem ser especificadas em lógica temporal (ao contrário de metas estendidas mais gerais, que podem requerer o uso de linguagens extra-lógicas especializadas e algoritmos ad hoc relacionados (e.g., [Lago et al., 2002])).

\subsubsection{Problemas e soluções}

Nesse capítulo, adotamos a definição de domínio de planejamento qualitativo apresentada na Seção 3.2 (pág. 38).

Definição 5.2. Um problema de planejamento para meta de alcançabilidade estendida é definido por uma tupla $\mathcal{P}=\left\langle\mathcal{D}, s_{0},\left(\varphi_{1}, \varphi_{2}\right)\right\rangle$, onde:

- $\mathcal{D}=\langle\mathcal{S}, \mathcal{L}, \mathcal{T}\rangle$ é um domínio de planejamento qualitativo com assinatura $(\mathbb{P}, \mathbb{A})$;

- $s_{0} \in \mathcal{S}$ é o estado inicial do ambiente;

- $\left(\varphi_{1}, \varphi_{2}\right)$ é uma meta de alcançabilidade estendida, definida sobre $\mathbb{P}$.

Definição 5.3. Seja $\pi$ (com estrutura de execução $\mathcal{D}_{\pi}$ ) uma política para um problema de planejamento qualitativo com meta de alcançabilidade estendida $\mathcal{P}=\left\langle\mathcal{D}, s_{0},\left(\varphi_{1}, \varphi_{2}\right)\right\rangle$. Dizemos que $\pi$ é uma solução:

- fraca para $\mathcal{P}$ se algum caminho a partir de $s_{0}$, em $\mathcal{D}_{\pi}$, alcança um estado que satisfaz $\varphi_{2}$ e passa apenas por estados que satisfazem $\varphi_{1}$;

- forte para $\mathcal{P}$ se todo caminho a partir de $s_{0}$, em $\mathcal{D}_{\pi}$, alcança um estado que satisfaz $\varphi_{2}$, após um número finito de passos, e passa apenas por estados que satisfazem $\varphi_{1}$;

- forte-cíclica para $\mathcal{P}$ se todo caminho a partir de $s_{0}$, em $\mathcal{D}_{\pi}$, alcança um estado que satisfaz $\varphi_{2}$ e passa apenas por estados que satisfazem $\varphi_{1}$. 
Devemos salientar que, mesmo para problemas de planejamento com metas de alcançabilidade estendidas, a qualidade da solução ainda é uma decisão do agente. A questão que surge é: seria possível definir a qualidade da solução desejada na própria especificação de meta? Ou seja, seria possivel escrever uma fórmula que expressasse, além das propriedades a serem preservadas e alcançadas, a qualidade da solução desejada?

\subsubsection{Subclasses de metas de alcançabilidade estendidas}

Algumas variações interessantes de metas de alcançabilidade estendidas são:

- $\left(\mathrm{T}, \varphi_{2}\right)$ : alcançar a propriedade $\varphi_{2}$ (meta de alcançabilidade simples);

- $\left(\varphi_{1}, \varphi_{2}\right)$ : alcançar a propriedade $\varphi_{2}$, preservando a propriedade $\varphi_{1}$;

- $\left(\neg \varphi_{1}, \varphi_{2}\right)$ : alcançar a propriedade $\varphi_{2}$, evitando a propriedade $\varphi_{1}$;

- $\left(\varphi_{1} \wedge \neg \varphi_{1}^{\prime}, \varphi_{2}\right)$ : alcançar a propriedade $\varphi_{2}$, preservando $\varphi_{1}$ e evitando $\varphi_{1}^{\prime}$.

Uma meta de alcançabilidade estendida $\left(\varphi_{1}, \varphi_{2}\right)$ define uma classe de metas que pode ser subdividida em duas subclasses distintas, de acordo com o tipo da fórmula $\varphi_{1}$ :

- quando $\varphi_{1}$ é uma fórmula proposicional, temos a subclasse linear de metas de alcançabilidade estendidas; pois a validade de $\varphi_{1}$ depende apenas do caminho específico que leva a um estado meta (i.e., um estado que satisfaz a fórmula $\varphi_{2}$ );

- quando $\varphi_{1}$ é uma fórmula temporal (i.e., uma fórmula contendo um operador temporal), temos a subclasse ramificada de metas de alcançabilidade estendidas; pois a validade de $\varphi_{1}$ depende não apenas do caminho específico que leva a um estado meta (i.e., um estado que satisfaz a fórmula $\varphi_{2}$ ), mas também das possíveis ramificações desse caminho, resultantes da execução de ações distintas daquela efetivamente escolhida pelo agente para alcançar um estado meta.

\subsection{Inadequação da lógica $C T L$}

Como vimos na Seção 2.2.2, CTL [Clarke \& Emerson, 1982] é uma lógica de tempo ramificado que permite raciocínio sobre linhas alternativas de tempo (i.e., futuros possíveis) e, portanto, essa lógica parece ser uma escolha bastante "natural" para tratar problemas de planejamento em ambientes não-determinísticos. De fato, CTL tem sido o principal formalismo usado para planejamento qualitativo baseado em verificação de modelos [Cimatti et al., 1997, Cimatti et al., 1998, 
Daniele et al., 1999, Pistore et al., 2001, Pistore \& Traverso, 2001]. Apesar disso, entretanto, a semântica de CTL não é adequada para tratar a classe de metas de alcançabilidade estendidas.

\subsubsection{Subclasse linear de metas de alcançabilidade estendidas}

Apesar de podermos utilizar CTL para especificar metas da subclasse linear, assim como para validar políticas para esse tipo de meta, sua semântica não é adequada para formalizar algoritmos para síntese de políticas para metas de alcançabilidade estendidas.

Usando CTL, uma meta de alcançabilidade estendida da subclasse linear (i.e., cuja condição a ser preservada $\varphi_{1}$ é especificada por uma fórmula proposicional) pode ser especificada de três formas distintas, dependendo da qualidade de solução desejada:

- $\exists\left(\varphi_{1} \sqcup \varphi_{2}\right)$, se uma solução fraca é desejada;

- $\forall\left(\varphi_{1} \sqcup \varphi_{2}\right)$, se uma solução forte é desejada; ou

- $\forall \square \exists\left(\varphi_{1} \sqcup \varphi_{2}\right)$, se uma solução forte-cíclica é desejada;

Note que, usando essas fórmulas, estamos especificando não apenas a meta de planejamento, mas também a qualidade de solução desejada (que decorre diretamente da semântica de CTL). Além disso, se $\varphi_{1}$ é a constante $T$, uma meta de alcançabilidade estendida da subclasse linear reduz-se a uma meta de alcançabilidade simples; podendo ser especificada como $\exists \diamond \varphi_{2}, \forall \diamond \varphi_{2}$, ou $\forall \square \exists \diamond \varphi_{2}$, respectivamente, para solução fraca, forte ou forte-cíclica (vide Seção 4.2.1).

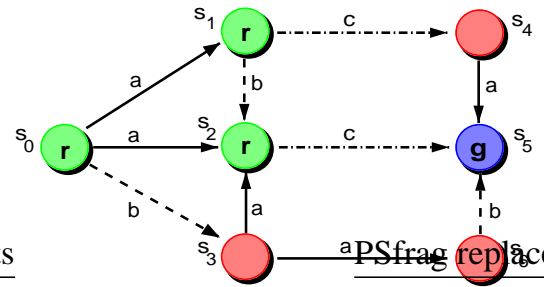

(a) domínio

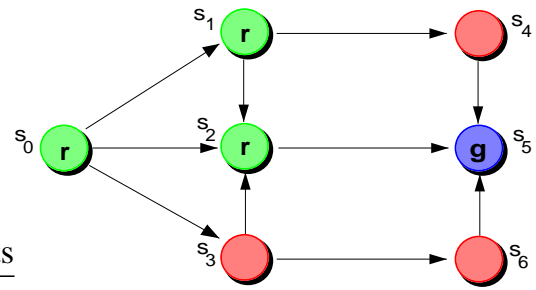

(b) estrutura de Kripke

Figura 5.3: Domínio de planejamento $\mathcal{D}^{1}$ e estrutura de Kripke $\mathcal{K}\left(\mathcal{D}^{1}\right)$ correspondente.

Para ver por que a semântica de CTL não é adequada para formalizar algoritmos de síntese para a subclasse linear de metas de alcançabilidade estendidas, considere o Exemplo 5.3 a seguir. 
Exemplo 5.3 (Síntese). Suponha que o agente no domínio de planejamento $\mathcal{D}^{1}$, ilustrado na Figura 5.3-a, esteja inicialmente no estado $s_{0}$ e que sua meta seja alcançar garantidamente um estado que satisfaça a propriedade g, passando apenas por estados que satisfaçam a propriedade $r$. É fácil ver que, de acordo com a semântica de CTL, essa meta de alcançabilidade estendida linear pode ser especificada pela fórmula $\forall(r \sqcup g)$; que vale num determinado estado somente se todo caminho que parte desse estado finalmente alcança $\mathrm{g}$, preservando $r$ ao longo de toda a sua extensão. Entretanto, de acordo com essa mesma semântica, também é claro que

$\left(\mathcal{K}\left(\mathcal{D}^{1}\right), s_{0}\right) \not \forall(r \sqcup g)$,

pois em $\mathcal{K}\left(\mathcal{D}^{1}\right)$, a estrutura de Kripke correspondente ao domínio $\mathcal{D}^{1}$ (Figura 5.3-b), as transições não têm rótulos e, portanto, não há como diferenciá-las com relação às ações que as produzem; além disso, existe uma transição que leva de $s_{0}$ a $s_{3}$, onde a propriedade $r$ não é satisfeita. Isso significa que, a partir do domínio de planejamento $\mathcal{D}^{1}$, um planejador baseado em CTL não seria capaz de sintetizar uma política que alcançasse a meta especificada pela fórmula $\forall(r \sqcup g)$; e, conseqüentemente, tal planejador terminaria devolvendo fracasso.

Para contornar essa limitação de CTL, quando utilizada para síntese de políticas, planejadores baseados em verificação de modelos freqüentemente utilizam algoritmos especializados (e.g., EAGLE [Lago et al., 2002]) para construir uma política (que induz um subgrafo do domínio de planejamento $\mathcal{D}$ ) e, em seguida, usam a semântica de CTL para garantir que a estrutura de execução dessa política satisfaz a fórmula CTL que especifica a meta de planejamento.

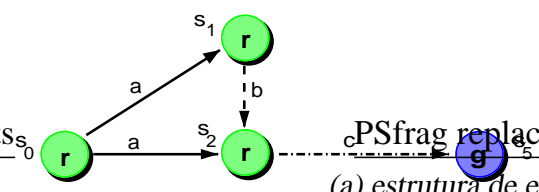

(a) estrutura de execução

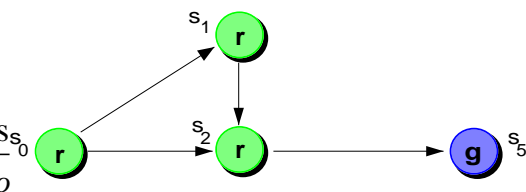

(b) estrutura de Kripke

Figura 5.4: Estrutura de execução $\mathcal{D}_{1}^{\pi_{1}}$ e estrutura de Kripke $\mathcal{K}\left(\mathcal{D}_{1}^{\pi_{1}}\right)$ correspondente.

Exemplo 5.4 (Validação). Considere a política $\pi_{1}=\left\{\left(s_{0}, a\right),\left(s_{1}, b\right),\left(s_{3}, c\right)\right\}$, cuja estrutura de execução no domínio $\mathcal{D}^{1}$ (Figura 5.3) é apresentada na Figura 5.4-a. É fácil ver que podemos usar a semântica de CTL para validar essa política. Basta verificar se a estrutura de Kripke correspondente à estrutura de execução dessa política (Figura 5.4-b) é um modelo temporal para a fórmula que especifica a meta de planejamento, ou seja, basta resolver o problema de verificação de modelos $\left(\mathcal{K}\left(\mathcal{D}_{\pi_{1}}^{1}\right), s_{0}\right) \stackrel{?}{\vDash} \forall(r \sqcup g)$. 
Conforme podemos ver pelos Exemplos 5.3 e 5.4, apesar de CTL ser adequada para especificar metas de alcançabilidade estendidas da subclasse linear, assim como para formalizar algoritmos de validação das soluções obtidas para elas, a semântica de CTL não é adequada para formalizar algoritmos para síntese de políticas para essa subclasse de metas.

\subsubsection{Subclasse ramificada de metas de alcançabilidade estendidas}

Nessa subseção, mostramos que a semântica da lógica CTL não é adequada para especificar metas de alcançabilidade estendidas da subclasse ramificada; nem para formalizar algoritmos de síntese ou validação de políticas para essas metas

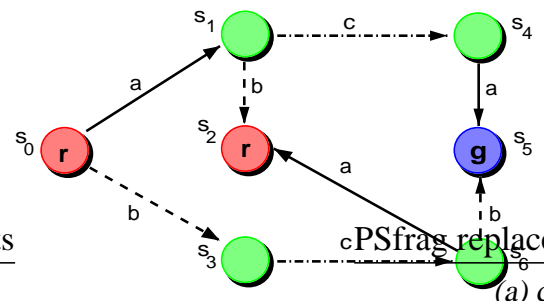

(a) domínio

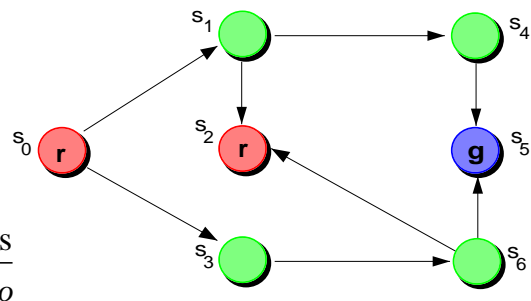

(b) estrutura de Kripke

Figura 5.5: Domínio de planejamento $\mathcal{D}^{2}$ e estrutura de Kripke $\mathcal{K}\left(\mathcal{D}^{2}\right)$ correspondente.

A subclasse ramificada de metas de alcançabilidade estendidas contém metas em que a condição de preservação $\varphi_{1}$ é uma fórmula temporal. Por exemplo, considere o domínio de planejamento $\mathcal{D}^{2}$, ilustrado na Figura 5.5-a. Nesse domínio, o agente poderia ser o robô Roomba do Exemplo 1.7 (pág. 11), a proposição $r$ poderia descrever a propriedade dos estados onde há estações de recarga de bateria e a propriedade $g$ poderia descrever a propriedade do estado final que o robô deseja alcançar. Nesse contexto, suponha que a meta do agente, que encontra-se inicialmente no estado $s_{0}$, seja a seguinte:

"necessariamente alcançar um estado que satisfaça a propriedade g, passando apenas por estados a partir dos quais uma estação de recarga de bateria possa ser alcançada, garantidamente, em no máximo dois passos".

A princípio, poderíamos pensar em usar a fórmula CTL $\forall((r \vee \forall O r \vee \forall O \forall O r) \sqcup g)$ para especificar essa meta. Entretanto, há dois problemas com essa especificação de meta: 
1. Uma vez que CTL não é capaz de distinguir entre os diferentes tipos de transições que podem ocorrer a partir de um determinado estado do modelo, não há como utilizar sua semântica para raciocinar sobre ramificações alternativas induzidas por ações que não serão efetivamente executadas. Entretanto, a condição de preservação $(r \vee \forall \circ r \vee \forall O \forall O r)$ é apenas uma contingência; ela não requer que o agente realmente alcance um estado onde haja uma estação de recarga de bateria (a menos que isso se torne necessário). Assim, é claro que a semântica da fórmula $\forall((r \vee \forall \bigcirc r \vee \forall \bigcirc \forall \bigcirc r) \sqcup g)$ não especifica exatamente a restrição que desejamos impor ao especificar a nossa meta de planejamento.

2. De qualquer forma, mesmo assumindo que a fórmula $\forall((r \vee \forall \bigcirc r \vee \forall \bigcirc \forall \bigcirc r) \sqcup g)$ possa ser usada para especificar a meta considerada, podemos ver que $\left(\mathcal{K}\left(\mathcal{D}^{2}\right), s_{0}\right) \not \forall((r \vee \forall \circ r \vee \forall \circ \forall \circ r) \sqcup g)$ pois, na estrutura de Kripke $\mathcal{K}\left(\mathcal{D}^{2}\right)$, ilustrada na Figura 5.5-b, não há garantia de que uma estação de recarga possa ser alcançada em no máximo dois passos, em todos os estados nos caminhos que partem do estado inicial $s_{0}$ (e.g., a partir de $s_{4}$ não há como alcançar uma estação de recarga). Note, porém, que:

- há uma estação de recarga no estado $s_{0}$;

- a partir do estado $s_{3}$, a estação de recarga em $s_{2}$ pode ser alcançada em dois passos;

- a partir do estado $s_{6}$, a estação de recarga em $s_{2}$ pode ser alcançada em um passo.

Portanto, a política $\pi_{2}=\left\{\left(s_{0}, b\right),\left(s_{3}, c\right),\left(s_{6}, b\right)\right\}$ é, de fato, uma solução para o problema de planejamento que estamos considerando. Apesar disso, entretanto, temos que $\left(\mathcal{K}\left(\mathcal{D}_{\pi_{2}}^{2}\right), s_{0}\right) \not \forall((r \vee \forall \circ r \vee \forall \circ \forall \circ r) \sqcup g)$,

ou seja, a estrutura de Kripke $\mathcal{K}\left(\mathcal{D}_{\pi_{2}}^{2}\right)$ na Figura 5.6-b, correspondente à estrutura de execução da política $\pi_{2}$ (Figura 5.6-a), não satisfaz essa meta; já que o estado $s_{2}$ (onde encontra-se a estação de recarga) não aparece nessa estrutura.

Assim, é evidente que a semântica da lógica CTL não é adequada para tratar esse problema de planejamento: nem para a síntese da política $\pi_{2}$ (a partir do domínio de planejamento $\mathcal{D}^{2}$ ), nem para a sua validação (a partir da sua estrutura de execução $\mathcal{D}_{\pi_{2}}^{2}$ ). Devemos ainda salientar que, como o estado $s_{2}$ (onde há uma estação de recarga) precisa ser descartado durante a síntese da política $\pi_{2}$, na verdade, não há mesmo como validar a estrutura de execução de $\pi_{2}$ após a sua síntese (pois a sua validação depende da existência de $s_{2}$ ). Isso mostra a importância de garantir a qualidade das políticas para metas de alcançabilidade estendidas durante o processo de síntese. 


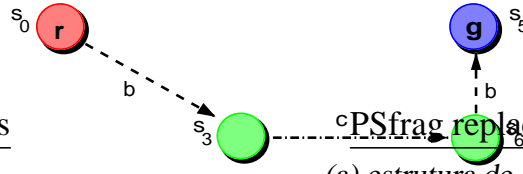

(a) estrutura de execução

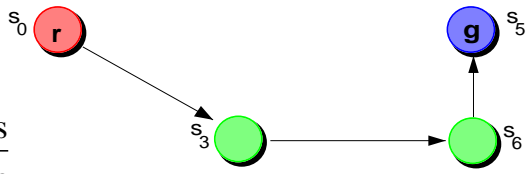

(b) estrutura de Kripke

Figura 5.6: Estrutura de execução $\mathcal{D}_{\pi_{2}}^{2}$ e estrutura de Kripke $\mathcal{K}\left(\mathcal{D}_{\pi_{2}}^{2}\right)$ correspondente.

\subsection{A lógica temporal $\alpha$-CTL}

Nessa seção, propomos uma nova versão da lógica temporal CTL, que denominamos $\alpha$-CTL, em cuja semântica as ações têm um papel fundamental [Pereira \& Barros, 2007c]. Na próxima seção, essa lógica será utilizada como base para a implementação de um arcabouço de planejamento sob incerteza qualitativa para metas de alcançabilidade estendidas

\subsubsection{Sintaxe de $\alpha$-CTL}

Para diferenciar sintaticamente as fórmulas $\alpha$-CTL (daquelas em CTL), empregamos um conjunto diferenciado de símbolos "pontuados" para representar os operadores temporais: $\odot$ (sucessor imediato $), \odot($ invariantemente $), \diamond($ finalmente $)$ e $\sqcup$ (até que).

Definição 5.4. Seja $p \in \mathbb{P}$ uma proposição atômica. A sintaxe de $\alpha$-CTL é definida indutivamente como:

$\varphi::=p|\neg p| \varphi_{1} \wedge \varphi_{2}\left|\varphi_{1} \vee \varphi_{2}\right| \exists \odot \varphi_{1}\left|\forall \odot \varphi_{1}\right| \exists \odot \varphi_{1}\left|\forall \odot \varphi_{1}\right| \exists\left(\varphi_{1} \sqcup \varphi_{2}\right) \mid \forall\left(\varphi_{1} \sqcup \varphi_{2}\right)$ •

De acordo com a sintaxe de $\alpha$-CTL, fórmulas bem-formadas estão na forma normal negativa; onde o escopo da negação é restrito a fórmulas atômicas. Isso facilita a definição da semântica dos operadores temporais globais em termos de pontos-fixos máximos e mínimos. Além disso, todos os operadores temporais devem ser prefixados por um quantificador de caminhos $(\exists$ ou $\forall$ ).

Os operadores temporais compostos pelo símbolo $\odot$ são definidos como:

$$
\begin{array}{lll}
\exists \odot \varphi_{2} & \doteq \quad \exists\left(T \sqcup \varphi_{2}\right) \\
\forall \odot \varphi_{2} & \doteq \quad \forall\left(\top \sqcup \varphi_{2}\right)
\end{array}
$$


Apesar das ações serem essenciais na definição da semântica de $\alpha$-CTL, note que elas não são usadas na composição das fórmulas dessa lógica. Quando especificamos metas de alcançabilidade estendidas, desejamos impor restrições apenas sobre os estados visitados durante a execução da política que será sintetizada. Impor restrições também sobre ações (além de desnecessário para a classe de metas em que estamos interessados) pode aumentar a complexidade de planejamento. Por essa razão, outras lógicas de ações encontradas na literatura [Nicola \& Vaandrager, 1990, Pecheur \& Raimondi, 2006], que permitem impor restrições sobre ações, não são adequadas para formalizar a classe de metas de alcançabilidade estendidas que propomos nessa tese.

\subsubsection{Semântica de $\alpha$-CTL}

Seja $\mathbb{P} \neq \varnothing$ um conjunto de proposições atômicas e $\mathbb{A} \neq \varnothing$ um conjunto de ações, contendo a ação trivial $\tau$. Um modelo temporal para uma fórmula da lógica $\alpha$-CTL é um grafo de transições de estados $\mathcal{D}=\langle\mathcal{S}, \mathcal{L}, \mathcal{T}\rangle$ com assinatura $(\mathbb{P}, \mathbb{A})$, cujos estados são rotulados por subconjuntos nãovazios de $\mathbb{P}$ e cujas transições são rotuladas por elementos de $\mathbb{A}$. Nesse modelo temporal, estados terminais (i.e., estados onde só é possível executar a ação $\tau$ ) persistem infinitamente no tempo. Informalmente, um modelo temporal para fórmulas $\alpha$-CTL é um domínio de planejamento $\mathcal{D}$ ou a estrutura de execução $\mathcal{D}_{\pi}$ de uma política $\pi$.

Intuitivamente, um estado $s$ em um modelo temporal $\mathcal{D}$ satisfaz uma fórmula $\forall \odot \varphi$ (ou $\exists \odot \varphi$ ) se existe uma ação $\alpha \in \mathbb{A}$ que, quando executada em $s$, necessariamente (ou possivelmente) alcança um estado sucessor imediato de $s$ que satisfaz a fórmula $\varphi$. Em outras palavras, a modalidade $\odot$ representa o conjunto de $\alpha$-sucessores de $s$, para alguma ação particular $\alpha$; o quantificador $\forall$ requer que todos esses $\alpha$-sucessores satisfaçam $\varphi$; e o quantificador $\exists$ requer que algum desses $\alpha$-sucessores satisfaça $\varphi$.

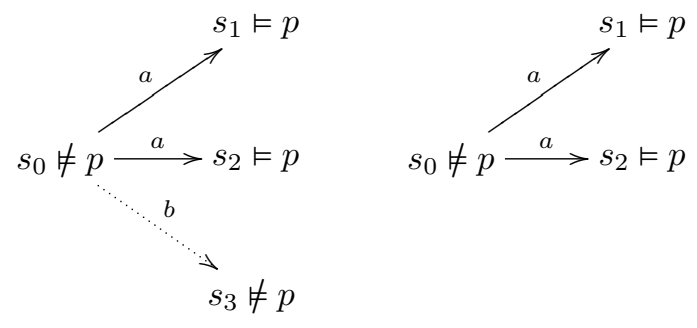
(a) domínio $\mathcal{D}^{3}$
(b) política $\pi_{3}=\left\{\left(s_{0}, a\right)\right\}$

Figura 5.7: Domínios e políticas como modelos temporais. 
70CAPÍTULO 5. PLANEJAMENTO PARA METAS DE ALCANÇABILIDADE ESTENDIDAS

Considere o modelo temporal $\mathcal{D}^{3}$, esquematizado na Figura 5.7-a. Nesse modelo, $\mathcal{T}\left(s_{0}, a\right)=$ $\left\{s_{1}, s_{2}\right\}$ e tanto $s_{1}$ quanto $s_{2}$ satisfazem $p$. Assim, de acordo com a semântica de $\alpha$-CTL, segue que $\left(\mathcal{D}^{3}, s_{0}\right) \vDash \forall \odot p$ (note que, de acordo com a semântica de CTL, o resultado seria diferente). Ainda de acordo com a semântica de $\alpha$-CTL, segue também que $\left(\mathcal{D}^{3}, s_{0}\right) \vDash \forall \odot \neg p$ (basta executar a ação $b$ no estado $s_{0}$ ). Isso é possível devido ao fato de que cada ocorrência da modalidade $\odot$ pode instanciar uma ação $\alpha$ distinta e, conseqüentemente, a quantificação pode ser feita sobre diferentes conjuntos de $\alpha$-sucessores de $s_{0}$. Entretanto, o fato de termos $\left(\mathcal{D}^{3}, s_{0}\right) \vDash \forall \odot p \wedge \forall \odot \neg p$ não significa que exista uma política capaz de alcançar $p$ e $\neg p$, simultaneamente, a partir do estado $s_{0}$ (tal política existiria apenas se fosse garantido alcançar $p$ e $\neg p$ em um único passo $\forall \odot(p \wedge \neg p)-$ mas, evidentemente, isso não é possível $\left.-\left(\mathcal{D}^{3}, s_{0}\right) \not \forall \odot(p \wedge \neg p)\right)$. O fato de termos $\left(\mathcal{D}^{3}, s_{0}\right) \vDash \forall \odot p \wedge \forall \odot \neg p$ significa apenas que, a partir de $s_{0}$, o agente pode escolher entre alcançar $p$ ou $\neg p$ no próximo estado do ambiente. Essa possibilidade de escolha é muito importante em planejamento; pois, se o agente não puder fazer escolhas, não há o que planejar. Num domínio de planejamento, o agente deve ser capaz de modificar o estado corrente do ambiente (i.e., das proposições que descrevem esse estado), selecionando e executando ações apropriadas, de acordo com a sua meta.

Antes de definirmos formalmente a semântica de $\alpha$-CTL, precisamos definir o conceito de pré-imagem de um conjunto de estados:

Definição 5.5. Seja $Y \subseteq \mathcal{S}$ um conjunto de estados. A pré-imagem fraca de $Y$, denotada por $\mathcal{T}_{\exists}^{-}(Y)$, é o conjunto $\{s \in \mathcal{S}: a \in \mathbb{A} e \mathcal{T}(s, a) \cap Y \neq \varnothing\}$; e a pré-imagem forte de $Y$, denotada por $\mathcal{T}_{\forall}^{-}(Y)$, é o conjunto $\{s \in \mathcal{S}: a \in \mathbb{A} e \varnothing \neq \mathcal{T}(s, a) \subseteq Y\}$.

A semântica para os operadores temporais globais $(\exists \bullet, \forall \bullet, \exists \bullet$ e $\forall \bullet)$ é derivada a partir da semântica dos operadores temporais locais $(\exists \odot$ e $\forall \odot)$, usando operações de ponto-fixo mínimo $(\mu)$ e ponto-fixo máximo $(\nu)$.

Definição 5.6. Sejam $\mathcal{D}=\langle\mathcal{S}, \mathcal{L}, \mathcal{T}\rangle$ um modelo temporal com assinatura $(\mathbb{P}, \mathbb{A})$ e $p \in \mathbb{P}$ uma proposição atômica. A intensão de uma fórmula $\alpha$-CTL $\varphi$ em $\mathcal{D}$ (i.e., o conjunto de estados de $\mathcal{D}$ que satisfazem $\varphi$ ), denotado por $\llbracket \varphi \rrbracket_{\mathcal{D}}$, é definida indutivamente como:

- $\llbracket p \rrbracket_{\mathcal{D}}=\{s \in \mathcal{S}: p \in \mathcal{L}(s)\}$

- $\neg p \rrbracket_{\mathcal{D}}=\mathcal{S} \backslash \llbracket p \rrbracket_{\mathcal{D}}$

- $\llbracket \varphi_{1} \wedge \varphi_{2} \rrbracket_{\mathcal{D}}=\llbracket \varphi_{1} \rrbracket_{\mathcal{D}} \cap \llbracket \varphi_{2} \rrbracket_{\mathcal{D}}$ 
- $\llbracket \varphi_{1} \vee \varphi_{2} \rrbracket_{\mathcal{D}}=\llbracket \varphi_{1} \rrbracket_{\mathcal{D}} \cup \llbracket \varphi_{2} \rrbracket_{\mathcal{D}}$

- $\llbracket \exists \odot \varphi_{1} \rrbracket_{\mathcal{D}}=\mathcal{T}_{\exists}^{-}\left(\llbracket \varphi_{1} \rrbracket_{\mathcal{D}}\right)$

- $\llbracket \forall \odot \varphi_{1} \rrbracket_{\mathcal{D}}=\mathcal{T}_{\forall}^{-}\left(\llbracket \varphi_{1} \rrbracket_{\mathcal{D}}\right)$

- $\exists \boxminus \varphi_{1} \rrbracket_{\mathcal{D}}=\nu Y .\left(\llbracket \varphi_{1} \rrbracket_{\mathcal{D}} \cap \mathcal{T}_{\exists}^{-}(Y)\right)$

- $\llbracket \forall \boxminus \varphi_{1} \rrbracket_{\mathcal{D}}=\nu Y \cdot\left(\llbracket \varphi_{1} \rrbracket_{\mathcal{D}} \cap \mathcal{T}_{\forall}^{-}(Y)\right)$

- $\llbracket \exists\left(\varphi_{1} \sqcup \varphi_{2}\right) \rrbracket_{\mathcal{D}}=\mu Y .\left(\llbracket \varphi_{2} \rrbracket_{\mathcal{D}} \cup\left(\llbracket \varphi_{1} \rrbracket_{\mathcal{D}} \cap \mathcal{T}_{\exists}^{-}(Y)\right)\right)$

- $\forall\left(\varphi_{1} \sqcup \varphi_{2}\right) \rrbracket_{\mathcal{D}}=\mu Y .\left(\llbracket \varphi_{2} \rrbracket_{\mathcal{D}} \cup\left(\llbracket \varphi_{1} \rrbracket_{\mathcal{D}} \cap \mathcal{T}_{\forall}^{-}(Y)\right)\right)$

Definição 5.7. Sejam $\mathcal{D}=\langle\mathcal{S}, \mathcal{L}, \mathcal{T}\rangle$ um modelo temporal com assinatura $(\mathbb{P}, \mathbb{A}), s \in \mathcal{S}$ um estado desse modelo e $\varphi$ uma fórmula $\alpha$-CTL. A relação de satisfatibilidade para $\alpha$-CTL é definida como:

$$
(\mathcal{D}, s) \vDash \varphi \Leftrightarrow s \in \llbracket \varphi \rrbracket_{\mathcal{D}}
$$

\subsubsection{Um verificador de modelos para $\alpha$-CTL}

Um verificador de modelos para $\alpha$-CTL pode ser implementado diretamente a partir da semântica de $\alpha$-CTL. Dados um modelo $\mathcal{D}=\langle\mathcal{S}, \mathcal{L}, \mathcal{T}\rangle$ com assinatura $(\mathbb{P}, \mathbb{A})$ e uma fórmula $\alpha$-CTL $\varphi$, o verificador VACTL ${ }^{1}$ [Pereira \& Barros, 2007d] inicia computando o conjunto de estados $C$ que não satisfazem a fórmula $\varphi$ no modelo $\mathcal{D}$; então, caso $C$ seja vazio, ele devolve sucesso; caso contrário, ele devolve esse conjunto como contra-exemplo.

$\operatorname{VACTL}(\varphi, \mathcal{D})$

$1 C \leftarrow \mathcal{S} \backslash \operatorname{InTENSÃO}(\varphi)$

2 se $C=\varnothing$ então devolva sucesso

3 devolva $C$

A operação básica desse verificador é implementada pela função $\operatorname{InTENSÃo}(\varphi)$, que computa indutivamente a intensão da fórmula $\varphi$ no modelo $\mathcal{D}$, usando a Definição 5.6. Nessa função, onde $\mathcal{D}$ é uma variável global, fórmulas proposicionais são tratadas por meio de recursividade direta; enquanto fórmulas temporais são tratadas por meio de recursividade indireta, implementada através de chamadas a funções auxiliares especializadas para cada um dos operadores temporais da lógica $\alpha$-CTL. A função InTENSÃo é definida conforme a seguir:

\footnotetext{
${ }^{1}$ Um protótipo desse verificador de modelos é apresentado no Apêndice B.
} 


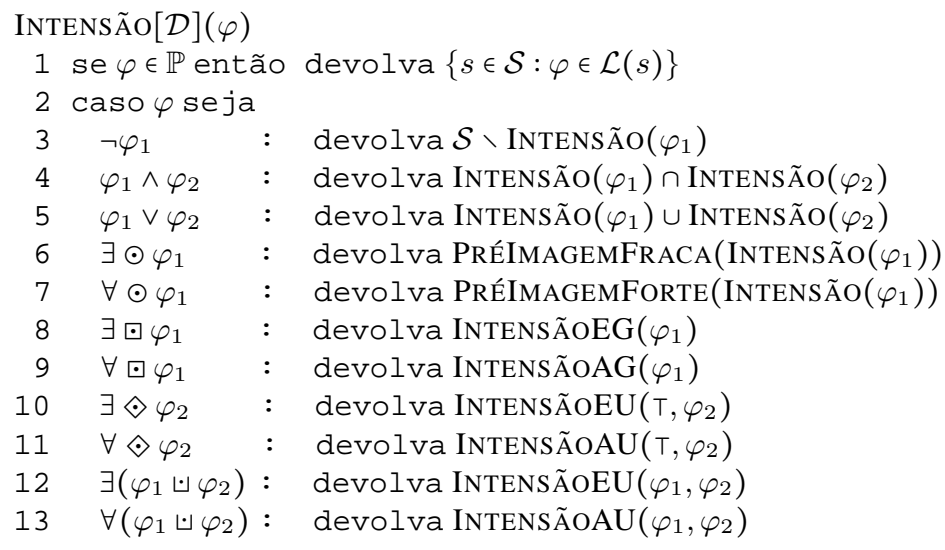

Tratamento de operadores temporais locais. Para verificar a validade de subfórmulas contendo operadores temporais locais $(\exists \odot \mathrm{e} \forall \odot)$, o algoritmo INTENS ÃO chama as seguintes funções básicas para computação de pré-imagens (que implementam diretamente a Definição 5.5):

PRÉIMAGEMFraCA $[\mathcal{D}](Y)$

1 devolva $\{s \in \mathcal{S}: a \in \mathbb{A}$ e $\mathcal{T}(s, a) \cap Y \neq \varnothing\}$

PRÉIMAGEMForte $[\mathcal{D}](Y)$

1 devolva $\{s \in \mathcal{S}: a \in \mathbb{A}$ e $\varnothing \neq \mathcal{T}(s, a) \subseteq Y\}$

Dado um conjunto de estados $Y \subseteq \mathcal{S}$ e um modelo $\mathcal{D}=\langle\mathcal{S}, \mathcal{L}, \mathcal{T}\rangle$, a função PréImagemFraca devolve um conjunto maximal de estados $X \subseteq \mathcal{S}$ tal que, para cada estado $s \in X$, existe uma ação $a \in \mathbb{A}$, cuja execução no estado $s$ leva a pelo menos um estado dentro do conjunto $Y$. Analogamente, a função PrélmagemForte devolve um conjunto maximal de estados $X \subseteq \mathcal{S}$ tal que, para cada estado $s \in X$, existe uma ação $a \in \mathbb{A}$, cuja execução no estado $s$ leva somente a estados dentro do conjunto $Y$.

Tratamento de operadores temporais globais. Para verificar a validade de subfórmulas contendo operadores temporais globais, o algoritmo INTENSÃo chama funções auxiliares capazes de computar pontos-fixos máximos e mínimos.

Os operadores temporais globais $\exists \boxminus$ e $\forall \varpi$, para os quais a semântica $\alpha$-CTL é definida em termos de ponto-fixo máximo, são tratados pelas seguintes funções: 


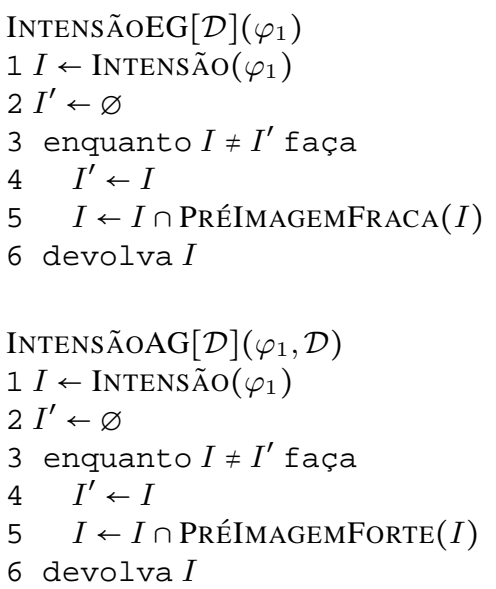

Essas duas funções são bastante semelhantes; sendo que a única diferença entre elas está no tipo de pré-imagem que elas computam dentro do laço de repetição. Inicialmente, o conjunto $I$ de estados que satisfazem a fórmula $\varphi_{1}$ no modelo $\mathcal{D}$ é computado. Em seguida, em cada iteração, o conjunto $I$ é atualizado de modo que apenas os estados para os quais existem ações que levam a estados que também satisfazem a fórmula $\varphi_{1}$ sejam mantidos. Tal atualização é realizada tomando-se a intersecção entre o conjunto $I$ e sua pré-imagem. Esse processo iterativo finaliza apenas quando um ponto-fixo máximo é obtido. Nesse caso, o conjunto de estados $I$ computado na última iteração corresponde à intensão da fórmula temporal originalmente considerada $\left(\exists \boxminus \varphi_{1}\right.$ ou $\left.\forall \bullet \varphi_{1}\right)$. Portanto, esse conjunto é devolvido como resposta pelas funções.

Os operadores temporais globais $\exists \sqcup$ e $\forall \bullet$, para os quais a semântica $\alpha$-CTL é definida em termos de ponto-fixo mínimo, são tratados pelas seguintes funções:

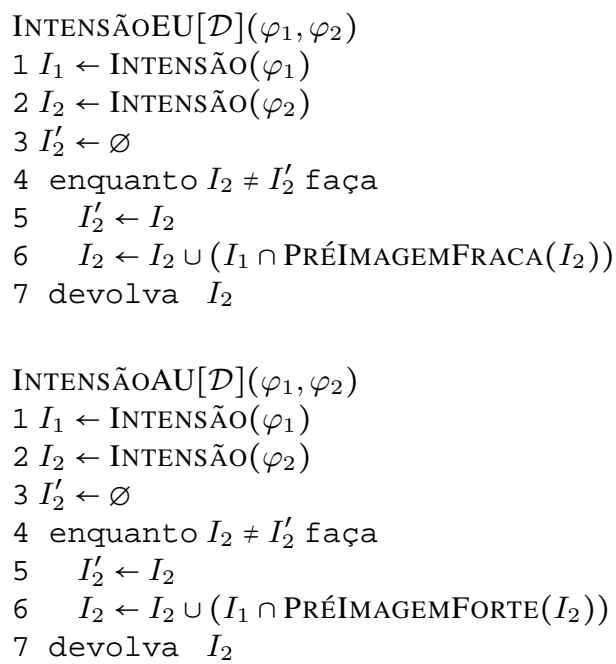


Essas duas funções também funcionam de forma semelhante; sendo que a única diferença entre elas está no tipo de pré-imagem que é computada dentro do laço de repetição. Inicialmente, elas computam os conjuntos $I_{1}$ e $I_{2}$ de estados que satisfazem as fórmulas $\varphi_{1}$ e $\varphi_{2}$, respectivamente. Em seguida, em cada iteração, elas computam a intersecção entre o conjunto $I_{1}$ e a pré-imagem do conjunto $I_{2}$ (para garantir que a fórmula $\varphi_{1}$ seja satisfeita em todos os estados dos caminhos que levam a estados onde $\varphi_{2}$ é satisfeita) e tomam a união desse resultado com o conjunto $I_{2}$ (para garantir que os estados que satisfazem $\varphi_{2}$, mas não $\varphi_{1}$, ainda possam ser considerados no conjunto final de estados que será devolvido como solução). Esse processo iterativo só termina quando um ponto-fixo mínimo é obtido. Nesse caso, o conjunto $I_{2}$ obtido na última iteração representa a intensão da fórmula temporal originalmente considerada $\left(\exists\left(\varphi_{1} \sqcup \varphi_{2}\right)\right.$ ou $\left.\forall\left(\varphi_{1} \sqcup \varphi_{2}\right)\right)$ e é devolvido como resposta pelas funções.

Propriedades formais do algoritmo VACTL. O teorema a seguir garante que o algoritmo de verificação de modelos VACTL é correto e completo. Sua prova é apresentada no Apêndice A.

Teorema 5.1. Dados um modelo $\mathcal{D}$ com assinatura $(\mathbb{P}, \mathbb{A})$ e uma fórmula $\alpha$-CTL $\varphi$, a função INTENSÃo $[\mathcal{D}](\varphi)$ devolve o conjunto de estados $\llbracket \varphi \rrbracket_{\mathcal{D}}$.

Corolário 5.1. Dados um modelo $\mathcal{D}$ com assinatura $(\mathbb{P}, \mathbb{A})$ e uma fórmula $\alpha$-CTL $\varphi$, o algoritmo $\operatorname{VACTL}(\mathcal{D}, \varphi)$ sucede se e somente se todo estado em $\mathcal{D}$ satisfaz $\varphi$.

\subsection{Planejamento baseado em $\alpha$-CTL}

A definição de intensão de uma fórmula $\alpha$-CTL $\varphi$ pode ser reformulada de modo que o conjunto de estados $\llbracket \varphi \rrbracket_{\mathcal{D}}$ seja transformado num submodelo $M \subseteq \mathcal{D}$, contendo não apenas os estados que satisfazem a fórmula $\varphi$, mas também as transições consideradas durante a seleção desses estados. Como veremos, tal submodelo $M$ nada mais é que a estrutura de execução de uma política $\pi$ capaz de alcançar a meta de planejamento especificada pela fórmula $\varphi$. Assim, um algoritmo de planejamento para metas de alcançabilidade estendidas expressas em $\alpha$-CTL [Pereira \& Barros, 2007b] pode ser formalizado por meio do uso dessa nova definição de $\llbracket \varphi \rrbracket_{\mathcal{D}}$.

Nessa seção, implementamos um arcabouço de planejamento para metas de alcançabilidade estendidas, tomando como base o verificador de modelos para $\alpha$-CTL apresentado na Subseção 5.3.3. Nesse arcabouço, uma solução para um problema de planejamento é obtida como efeito 
colateral da verificação de um modelo $\mathcal{D}$, especificando um ambiente de planejamento, com relação a uma fórmula $\alpha$-CTL $\varphi$, especificando uma meta de planejamento.

\subsubsection{O algoritmo de planejamento PACTL}

Uma meta de alcançabilidade estendida $\left(\varphi_{1}, \varphi_{2}\right)$ pode ser especificada por uma das três fórmulas $\alpha$-CTL a seguir, dependendo da qualidade de solução desejada:

- $\varphi \doteq \exists\left(\varphi_{1} \sqcup \varphi_{2}\right)$, se uma solução fraca é desejada;

- $\varphi \doteq \forall\left(\varphi_{1} \sqcup \varphi_{2}\right)$, se uma solução forte é desejada; ou

- $\varphi \doteq \forall \bullet \exists\left(\varphi_{1} \sqcup \varphi_{2}\right)$, se uma solução forte-cíclica é desejada.

Dado um problema de planejamento $\mathcal{P}=\left\langle\mathcal{D}, s_{0}, \varphi\right\rangle$ com assinatura $(\mathbb{P}, \mathbb{A})$, onde $\varphi$ é uma meta de alcançabilidade estendida especificada em $\alpha$-CTL, uma solução com a qualidade desejada para $\mathcal{P}$ pode ser obtida pelo algoritmo PACTL $^{2}$, definido conforme a seguir.

$\operatorname{PACTL}(\mathcal{P})$

$1 M \leftarrow \operatorname{Modelo}(\min , \varphi)$

$2 C \leftarrow \operatorname{Cobertura}(M)$

3 se $s_{0} \in C$ então devolva Política $(M)$

4 devolva fracasso

Esse algoritmo inicia sintetizando um submodelo $M \subseteq \mathcal{D}$ e obtendo o conjunto $C$ de estados cobertos por esse submodelo. Então, se $s_{0} \in C$, ele devolve uma política $\pi$ extraída de $M$, cuja execução permite ao agente alcançar a meta $\varphi$, a partir de $s_{0}$, no domínio $\mathcal{D}$; caso contrário, ele devolve fracasso (Figura 5.8).

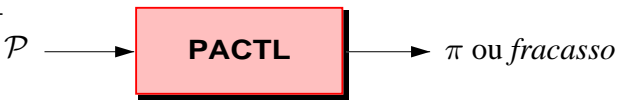

Figura 5.8: Planejador baseado em $\alpha$-CTL.

Para sintetizar o submodelo $M$, o algoritmo Pactl usa a função Modelo (vide Subseção 5.4.2), que devolve um conjunto de estados e pares de estados e ações. Para obter a cobertura desse modelo, PACTL faz uma chamada à seguinte função:

\footnotetext{
${ }^{2}$ Um protótipo desse planejador é apresentado no Apêndice B.
} 
Cobertura $(M)$

1 devolva $\{s \in \mathcal{S}: s \in M\} \cup\{s \in \mathcal{S}:(s, a) \in M\}$

que devolve a união dos conjuntos de estados terminais e não-terminais de $M$. Finalmente, para extrair uma política do submodelo $M$, o algoritmo PaCtL usa a função $\operatorname{PoLítica~}(M)$, que devolve uma política $\pi$ tal que:

- $\operatorname{Cobertura}(\pi)=\operatorname{Cobertura}(M) \mathrm{e}$

- para todo $(s, a) \in \pi$ e $\left(s^{\prime}, a^{\prime}\right) \in \pi$, se $s=s^{\prime}$, então $a=a^{\prime}$.

\subsubsection{Síntese de modelos}

Para sintetizar o modelo $M$, o algoritmo PACtL usa a função Modelo, para a qual a variável $\mathcal{D}$ é global. Essa função é muito similar à função InTENsÃo, utilizada pelo verificador de modelos VACTL (vide Subseção 5.3.3). A principal diferença é que a função Modelo requer um parâmetro adicional que indica se a fórmula $\varphi$ está num escopo min (que evita ciclos) ou max (que permite ciclos). Especialmente quando desejamos modelos para fórmulas contendo os operadores temporais globais $\exists \bullet$ e $\forall \bullet$ (que especificam propriedades que devem ser invariantes no tempo), esse parâmetro deve ser definido como max.

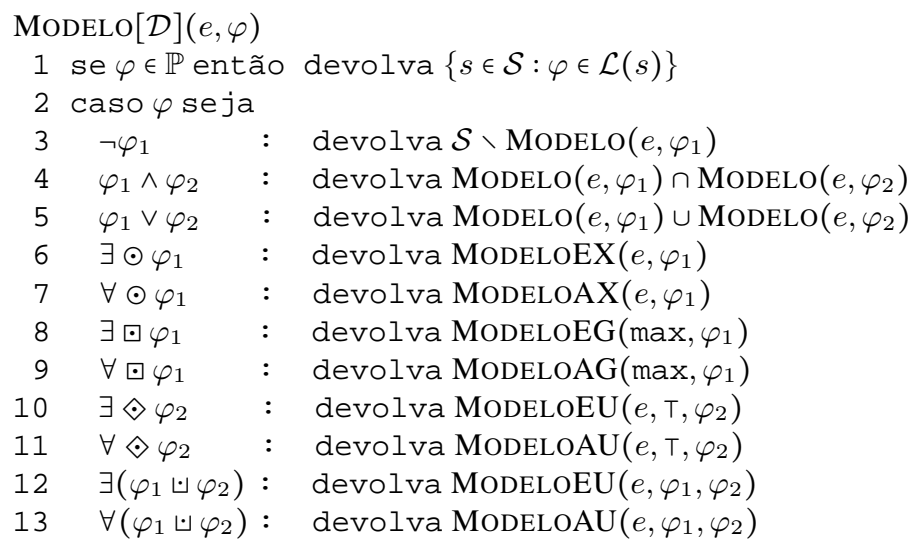

Tratamento de fórmulas proposicionais. Um modelo $M$ sintetizado a partir de uma fórmula proposicional $\varphi$ é o conjunto maximal de estados $\mathcal{Y} \subseteq \mathcal{S}$ tal que, para todo $s \in \mathcal{Y}, s \vDash \varphi$. Fórmulas proposicionais são tratadas diretamente pela função MODELO, usando indução estrutural. 
Tratamento de operadores temporais locais. Para tratar os operadores temporais $\exists \odot$ e $\forall \odot$, a função MODELo chama as seguintes funções auxiliares:

$\operatorname{ModeloEX}[\mathcal{D}]\left(e, \varphi_{1}\right)$

$1 M_{1} \leftarrow \operatorname{Modelo}\left(e, \varphi_{1}\right)$

$2 I_{1} \leftarrow$ PréimagemFracamap $\left(\operatorname{Cobertura}\left(M_{1}\right)\right)$

$3 P_{1} \leftarrow \operatorname{PodaX}\left(I_{1}, M_{1}\right)$

4 devolva $M_{1} \cup P_{1}$

$\operatorname{ModeLOAX}[\mathcal{D}]\left(e, \varphi_{1}\right)$

$1 M_{1} \leftarrow \operatorname{MODELO}\left(e, \varphi_{1}\right)$

$2 I_{1} \leftarrow$ PréImagemForteMap $\left(\right.$ Cobertura $\left.\left(M_{1}\right)\right)$

$3 P_{1} \leftarrow \operatorname{PodaX}\left(I_{1}, M_{1}\right)$

4 devolva $M_{1} \cup P_{1}$

As funções ModeloEX e ModeloAX sintetizam submodelos de $\mathcal{D}$, a partir de fórmulas do tipo $\exists \odot \varphi_{1}$ e $\forall \odot \varphi_{1}$, respectivamente. Para tanto, primeiro elas sintetizam um modelo $M_{1}$ a partir da subfórmula $\varphi_{1}$ e computam a pré-imagem $I_{1}$ do conjunto de estados cobertos por esse modelo; em seguida, elas podam o conjunto $I_{1}$ para evitar que estados já cobertos por $M_{1}$ sejam mapeados a novas ações (e.g., estado $s_{1}$ na Figura 5.9-a), obtendo um pré-componente $P_{1}$. Finalmente, a união do modelo $M_{1}$ com o pré-componente $P_{1}$ é devolvida como resposta. Os pares $(s, a) \in P_{1}$ é que garantem que uma política extraída do modelo $M_{1} \cup P_{1}$ satisfaz a meta especificada pela formula inicial $\left(\exists \odot \varphi_{1}\right.$ ou $\left.\forall \odot \varphi_{1}\right)$.

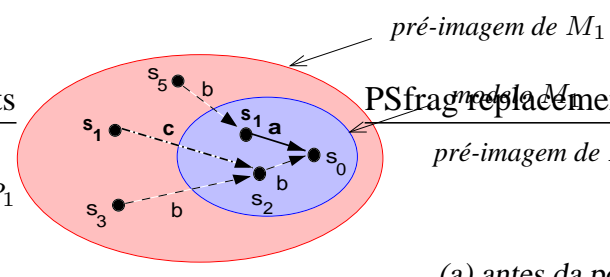

(a) antes da poda

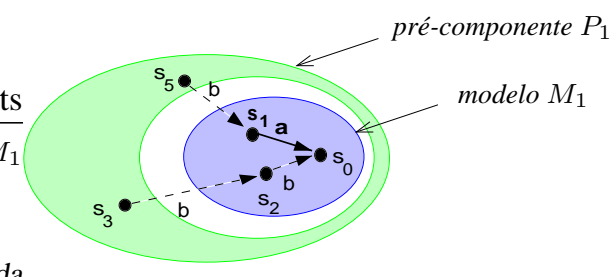

(b) depois da poda

Figura 5.9: Mecanismo de síntese de modelos para operadores temporais locais.

A computação de pré-imagens e poda é realizada pelas seguintes funções:

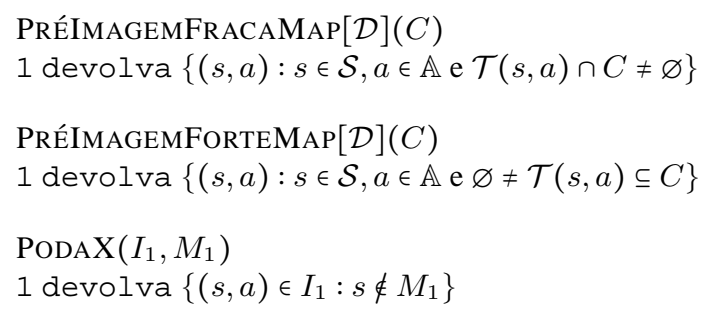


Tratamento de operadores temporais globais. Para tratar os operadores temporais globais $\exists \varpi$ e $\forall \varpi$, a função Modelo chama as seguintes funções de ponto-fixo máximo:
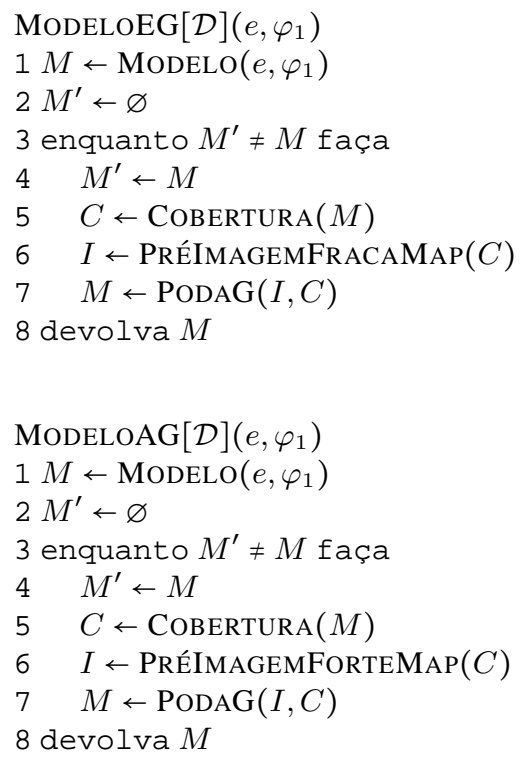

As funções Modeloeg e ModeloAg sintetizam submodelos de $\mathcal{D}$, a partir de fórmulas do tipo $\exists \boxminus \varphi_{1}$ e $\forall \bullet \varphi_{1}$, respectivamente. Para tanto, primeiro elas sintetizam um modelo $M$ a partir da subfórmula $\varphi_{1}$. A partir daí, iterativamente, esse modelo é contraído da seguinte forma: primeiro a pré-imagem $I$ do conjunto de estados cobertos pelo modelo $M$ corrente é computada e, em seguida, o conjunto $I$ é podado de modo que apenas estados cobertos pelo modelo $M$ sejam mantidos. Então, o resultado da poda passa a ser o modelo $M$ corrente na próxima iteração. Procedendo dessa forma, a cada iteração, estados que não satisfazem a propriedade invariante especificada por $\varphi_{1}$ são descartados. Assim, quando o ponto-fixo máximo é atingido, podemos garantir que uma política extraída do modelo $M$ computado na última iteração (e devolvido como resposta) satisfaz a meta especificada pela formula inicial $\left(\exists \bullet \varphi_{1}\right.$ ou $\left.\forall \bullet \varphi_{1}\right)$.

A poda utilizada pelas funções ModeloEG e ModeloAG é definida conforme a seguir:

$\operatorname{PodaG}(I, C)$

1 devolva $\{(s, a) \in I: s \in C\}$

Um exemplo de como funcionam o algoritmo de tratamento do operador temporal global $\forall \bullet$ pode ser visto na Figura 5.10. O tratamento para o operador $\exists \odot$ funciona de forma análoga. 


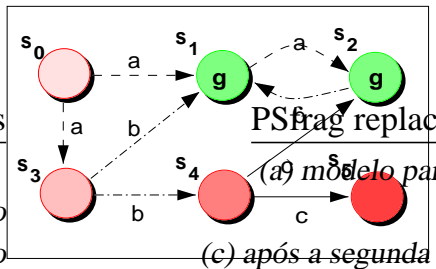

(a) modelo para $\exists \odot g$

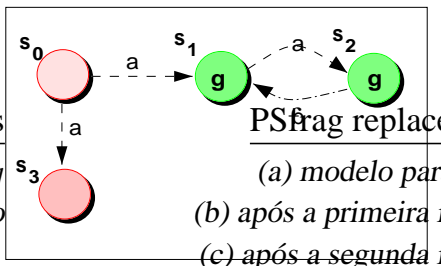

(c) após a segunda iteração

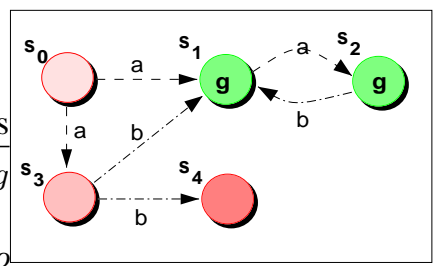

(b) após a primeira iteração

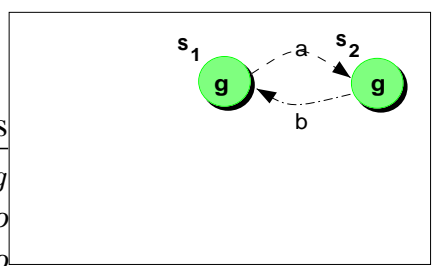

(d) após a terceira iteração

Figura 5.10: Mecanismo de síntese de modelos para a fórmula $\forall \bullet \exists \diamond g$.

Para tratar os operadores temporais globais $\exists \sqcup$ e $\forall \bullet$, a função Modelo chama as seguintes funções de ponto-fixo mínimo:

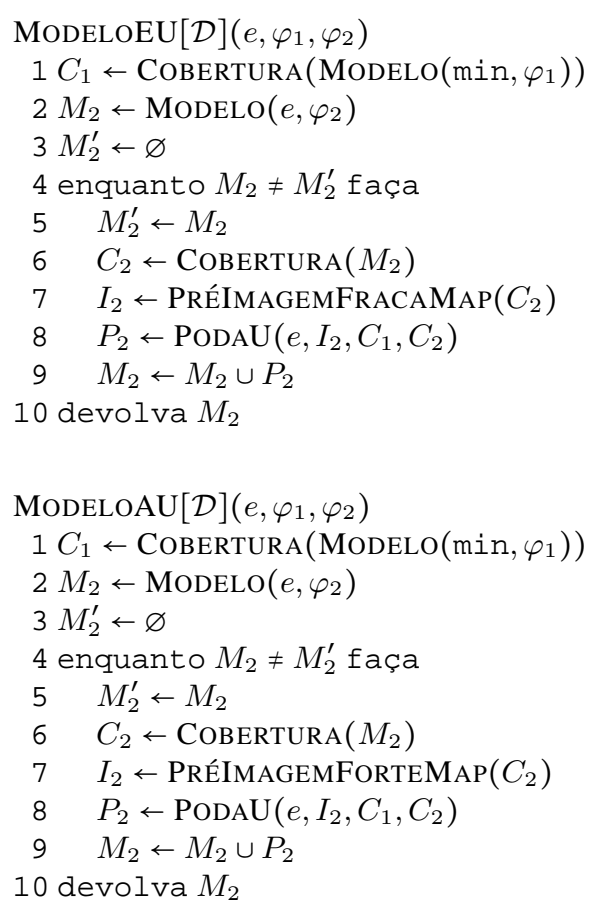


As funções ModeloEU e ModeloAU sintetizam submodelos de $\mathcal{D}$, a partir de fórmulas do tipo $\exists\left(\varphi_{1} \sqcup \varphi_{2}\right)$ e $\forall\left(\varphi_{1} \sqcup \varphi_{2}\right)$, respectivamente. Para tanto, primeiro elas determinam o conjunto de estados $C_{1}$, que satisfazem a fórmula $\varphi_{1}$, e sintetizam um modelo $M_{2}$ para a fórmula $\varphi_{2}$. A partir daí, iterativamente, o modelo $M_{2}$ é expandido da seguinte forma: primeiro a pré-imagem $I_{2}$ do conjunto de estados cobertos pelo modelo $M_{2}$ corrente é computada e, em seguida, o conjunto $I_{2}$ é podado de modo que apenas estados que também estejam em $C_{1}$ sejam mantidos no pré-componente $P_{2}$ (caso ciclos devam ser evitados $(e=\min$ ), a função de poda também elimina de $P_{2}$ todos os estados que já foram cobertos por $M_{2}$ ). Então, a união do modelo $M_{2}$ com o seu pré-componente $P_{2}$ passa a ser o novo modelo $M_{2}$ a ser considerado na próxima iteração. Procedendo dessa forma, a cada iteração, o modelo $M_{2}$ inicial é expandido com novos pares $(s, a)$, tais que $s \in C_{1}$ e a execução da ação $a$ no estado $s$ leva a estados em $M_{2}$. Assim, quando o ponto-fixo mínimo é atingido, podemos garantir que uma política extraída do modelo $M_{2}$ computado na última iteração (e devolvido como resposta) satisfaz a meta especificada pela formula inicial $\left(\exists\left(\varphi_{1} \bullet \varphi_{2}\right)\right.$ ou $\left.\forall\left(\varphi_{1} \bullet \varphi_{2}\right)\right)$.

A poda utilizada pelas funções ModeloEU e ModeloAU é definida conforme a seguir:

$\operatorname{PODAU}\left(e, I_{2}, C_{1}, C_{2}\right)$

$1 P_{2} \leftarrow\left\{(s, a) \in I_{2}: s \in C_{1}\right\}$

2 se $e=\min$ ent ão $P_{2} \leftarrow\left\{(s, a) \in P_{2}: s \notin C_{2}\right\}$

3 devolva $P_{2}$

\subsubsection{Propriedades formais do algoritmo PACTL}

Algumas propriedades formais do algoritmo PACTL são estabelecidas pelos teoremas a seguir, cujas provas são apresentadas no Apêndice A.

Teorema 5.2. Seja $\mathcal{P}=\left\langle\mathcal{D}, s_{0}, \exists\left(\varphi_{1} \sqcup \varphi_{2}\right)\right\rangle$ um problema de planejamento. Se $\mathcal{P}$ tem solução, então a política devolvida pelo algoritmo $\operatorname{PACTL}(\mathcal{P})$ é uma solução fraca para $\mathcal{P}$.

Teorema 5.3. Seja $\mathcal{P}=\left\langle\mathcal{D}, s_{0}, \forall\left(\varphi_{1} \sqcup \varphi_{2}\right)\right\rangle$ um problema de planejamento. Se $\mathcal{P}$ tem solução, então a política devolvida pelo algoritmo $\operatorname{PACTL}(\mathcal{P})$ é uma solução forte para $\mathcal{P}$.

Teorema 5.4. Seja $\mathcal{P}=\left\langle\mathcal{D}, s_{0}, \forall \bullet \exists\left(\varphi_{1} \sqcup \varphi_{2}\right)\right\rangle$ um problema de planejamento. Se $\mathcal{P}$ tem solução, então a política devolvida pelo algoritmo $\operatorname{PACTL}(\mathcal{P})$ é uma solução forte-cíclica para $\mathcal{P}$.

Teorema 5.5. Seja $\mathcal{P}=\left\langle\mathcal{D}, s_{0}, \varphi\right\rangle$ um problema de planejamento para uma meta de alcançabilidade estendida $\varphi$. Então, o algoritmo $\operatorname{PACTL}(\mathcal{P})$ devolve fracasso se e só se $\mathcal{P}$ não tem solução. 
Teorema 5.6. O menor caminho gerado pela execução de uma política $\pi$, obtida por meio da chamada $\operatorname{PACTL}\left(\left\langle\mathcal{D}, s_{0}, \exists\left(\varphi_{1} \sqcup \varphi_{2}\right)\right\rangle\right)$, é mínimo no melhor caso.

Teorema 5.7. O maior caminho gerado pela execução de uma política $\pi$, obtida por meio da chamada $\operatorname{PACTL}\left(\left\langle\mathcal{D}, s_{0}, \forall\left(\varphi_{1} \sqcup \varphi_{2}\right)\right\rangle\right)$, é mínimo no pior caso.

\subsection{Sumário}

Nesse capítulo, introduzimos uma classe de metas mais expressiva, que inclui metas de alcançabilidade simples como uma subclasse, que denominamos metas de alcançabilidade estendidas. Em seguida, mostramos que a semântica da lógica CTL [Clarke \& Emerson, 1982] é inadequada tanto para especificar metas dessa classe, quanto para formalizar algoritmos de síntese e validação de políticas para elas.

Como uma forma de resolver esse problema, definimos uma nova versão da lógica temporal de tempo ramificado CTL, que denominamos $\alpha$-CTL [Pereira \& Barros, 2007c]. Então, com base nessa lógica, implementamos um verificador de modelos [Pereira \& Barros, 2007d] que, em seguida, foi utilizado como base para a implementação de um arcabouço de planejamento para metas de alcançabilidade estendidas (em domínios qualitativos e completamente observáveis), capaz de sintetizar soluções com diferentes qualidades. Procedendo dessa forma, em vez de construir uma política de forma ad hoc e depois validá-la, podemos sintetizar uma política cuja validade é consequiência direta de um processo de síntese bem fundamentado em métodos formais.

No próximo capítulo, discutimos a qualidade das soluções obtidas quando consideramos domínios de planejamento quantitativos. 
82CAPÍTULO 5. PLANEJAMENTO PARA METAS DE ALCANÇABILIDADE ESTENDIDAS 


\section{Capítulo 6}

\section{Qualidade das soluções em domínios quantitativos}

Quando um agente toma decisões com base num modelo qualitativo, em geral, ele escolhe ações levando em conta apenas o fato de elas permitirem ou não que sua meta seja alcançada. Por outro lado, quando um agente dispõe de um modelo quantitativo, ele pode definir preferências entre os diferentes resultados das diversas ações possíveis e, depois, ponderar essas preferências levando em conta a probabilidade de ocorrência de cada resultado. Agindo dessa forma, o agente pode encontrar uma política que lhe permita ter um desempenho ótimo no caso esperado. Nesse capítulo, mostramos que, mesmo quando a política é ótima no caso esperado, ainda podemos classificá-la como fraca, forte-cíclica ou forte.

\subsection{Processos de decisão markovianos}

Um processo de decisão é uma seqüência de variáveis aleatórias $\left\langle S_{t}\right\rangle_{t=0}^{\infty}$, representando estados de um ambiente dinâmico estocástico, que induz uma função de transição probabilística da forma

$$
\mathcal{P}\left(S_{t+1}=s_{t+1} \mid S_{0}=s_{0}, \ldots, S_{t}=s_{t} ; A_{t}=a_{t}\right) .
$$

Essa função de transição probabilística define a probabilidade condicional do próximo estado do ambiente ser $s_{t+1}$, dado que o histórico de evolução desse ambiente é $s_{0}, \ldots, s_{t}$ e que, no seu estado corrente $s_{t}$, o agente escolheu executar a ação $a_{t}$. Porém, quando o estado corrente $s_{t}$ resume o histórico de evolução do ambiente de forma compacta, sem descartar informações 
necessárias para prever o estado futuro $s_{t+1}$, temos que:

$$
\mathcal{P}\left(S_{t+1}=s_{t+1} \mid S_{0}=s_{0}, \ldots, S_{t}=s_{t} ; A_{t}=a_{t}\right)=\mathcal{P}\left(S_{t+1}=s_{t+1} \mid S_{t}=s_{t} ; A_{t}=a_{t}\right)
$$

A Equação 6.2 especifica a propriedade de Markov [Markov, 1971] e um processo de decisão que apresenta essa propriedade é denominado processo de decisão markoviano (MDP - MARKOV Decision Process) [Puterman, 1994]. Em outras palavras, um processo de decisão markoviano é um processo de decisão em que a probabilidade de transição para um estado futuro depende apenas do estado corrente do ambiente e da ação executada nesse estado, sendo o passado do ambiente completamente irrelevante.

\subsubsection{Modelo do ambiente}

Um processo de decisão markoviano é um modelo formal para uma interação síncrona entre um agente e seu ambiente: a cada instante, o agente observa o estado corrente do ambiente $s_{t} \mathrm{e}$ decide executar uma ação $a_{t}$; essa ação afeta o estado corrente, produzindo um estado futuro $s_{t+1}$ e uma recompensa $r_{t+1}$ (Figura 6.1). O objetivo do agente é maximizar a soma esperada das recompensas recebidas ao longo do tempo [Puterman, 1994].

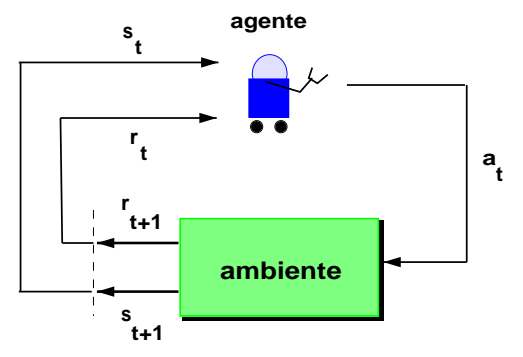

Figura 6.1: Interação entre agente e ambiente dinâmico estocástico.

Definição 6.1. Um processo de decisão markoviano (MDP) é definido por uma tupla da forma $\mathcal{M}=\langle\mathcal{S}, \mathcal{A}, \mathcal{T}, \mathcal{R}\rangle$, onde:

- $\mathcal{S} \neq \varnothing$ é um conjunto finito de estados possiveis do ambiente;

- $\mathcal{A} \neq \varnothing$ é um conjunto finito de ações executáveis pelo agente;

- $\mathcal{T}: \mathcal{S} \times \mathcal{A} \times \mathcal{S} \mapsto[0,1]$ é uma função de transição de estados probabilística; 
- $\mathcal{R}: \mathcal{S} \mapsto \mathbb{R}_{+}$é uma função de recompensa de estados.

Num MDP $\mathcal{M}=\langle\mathcal{S}, \mathcal{A}, \mathcal{T}, \mathcal{R}\rangle$, o conjunto $\mathcal{S}$ representa todos os possíveis estados em que o ambiente pode estar, enquanto o conjunto $\mathcal{A}$ representa todas as ações que o agente é capaz de executar. A função de transição $\mathcal{T}$ representa a dinâmica do ambiente, de modo que $\mathcal{T}\left(s, a, s^{\prime}\right)$ denota a probabilidade de transição para o estado $s^{\prime}$, dado que a ação $a$ foi executada no estado $s$; ou seja, para todo $s, s^{\prime} \in \mathcal{S}$ e $a \in \mathcal{A}$,

$$
\mathcal{T}\left(s, a, s^{\prime}\right)=\mathcal{P}\left(S_{t+1}=s^{\prime} \mid S_{t}=s ; A_{t}=a\right) .
$$

Ademais, a função de transição $\mathcal{T}$ também define uma distribuição de probabilidades sobre o conjunto de estados do ambiente $\mathcal{S}$; ou seja, para cada par $s \in \mathcal{S}$ e $a \in \mathcal{A}$, se para algum $s^{\prime} \in \mathcal{S}$ temos que $\mathcal{T}\left(s, a, s^{\prime}\right)>0$, então:

$$
\sum_{s^{\prime} \in \mathcal{S}} \mathcal{T}\left(s, a, s^{\prime}\right)=1
$$

Finalmente, a função de recompensa $\mathcal{R}$ produz um número real positivo $\mathcal{R}(s)$, representando a recompensa imediata que o agente recebe ao visitar o estado $s \in \mathcal{S}$.

\subsubsection{Modelo do agente}

Uma política é uma função $\pi: \mathcal{S} \mapsto \mathcal{A}$, que mapeia estados em ações; ou seja, $\pi(s)$ denota a ação recomendada pela política $\pi$ para o estado $s$. No contexto de MDPs, consideramos que uma política é sempre uma função total. Assim, quando um agente segue uma política $\pi$, não importa qual seja o resultado de uma ação, o agente sempre saberá o que fazer em seguida. Quando as ações recomendadas por uma política são sempre as mesmas para cada estado, independentemente do instante de tempo considerado, dizemos que a política é estacionária; do contrário, dizemos que a política é não-estacionária.

Dada uma política $\pi$, o agente decide o que fazer consultando sua percepção, que informa o estado corrente $s$, e depois executando a ação $\pi(s)$, conforme recomendado pela política $\pi$. Nesse sentido, podemos dizer que uma política é um modelo de um agente executor, i.e., um agente de reflexo simples, sintetizado a partir das decisões tomadas por uma agente decisório. 


\subsubsection{Políticas ótimas}

Devido à natureza estocástica do ambiente, a cada vez que uma política é executada a partir de um determinado estado inicial fixo, podemos observar um histórico distinto de evolução do ambiente. Assim, ao avaliarmos uma política, além de considerarmos por quantos passos essa política será seguida pelo agente, devemos também levar em conta a utilidade de cada um dos possíveis históricos que podem ser gerados por esses passos.

Há duas formas de avaliarmos uma política:

- Horizonte finito. Assumimos que o agente seguirá a política por um número finito de passos. Nesse caso, a maneira como o agente se comporta costuma mudar, à medida em que ele se aproxima de seus últimos passos. Assim, quando o tempo de vida do agente é finito, geralmente, a política é não-estacionária.

Seja $\pi$ uma política não-estacionária, i.e., uma política que associa o estado $s$ à ação $\pi_{n}(s)$, quando ainda restam $n$ passos ao agente; e seja $V_{n}^{\pi}(s)$ a recompensa total esperada, a partir do estado $s$, seguindo-se a política $\pi$ por $n$ passos. Definimos indutivamente o valor de $\pi$, para um estado $s$, da seguinte maneira [Bellman, 1957]:

$$
V_{n}^{\pi}(s)= \begin{cases}\mathcal{R}(s) & \text { se } n=0 \\ \mathcal{R}(s)+\sum_{s^{\prime} \in \mathcal{S}} \mathcal{T}\left(s, \pi_{n}(s), s^{\prime}\right) \cdot V_{n-1}^{\pi}\left(s^{\prime}\right) & \text { se } n>0\end{cases}
$$

- Horizonte infinito. Assumimos que o agente seguirá a política por um número infinito de passos. Nesse caso, como o agente tem sempre um número infinito de passos restantes, não há porque mudar o seu modo de agir com o passar do tempo. Então, quando consideramos um horizonte infinito, é mais razoável considerar também que $\pi$ é uma política estacionária. Ademais, para garantir que seu valor seja finito (apesar de ser dado por uma soma de infinitos termos), usaremos uma fator de desconto $0<\gamma<1$.

Seja $\pi$ uma política estacionária, que associa o estado $s$ à ação $\pi(s)$; e seja $V^{\pi}(s)$ a recompensa descontada total esperada, a partir do estado $s$, seguindo-se a política $\pi$ por infinitos passos. Definimos indutivamente o valor de $\pi$, para um estado $s$, como [Bellman, 1957]:

$$
V^{\pi}(s)=\mathcal{R}(s)+\gamma \sum_{s^{\prime} \in \mathcal{S}} \mathcal{T}\left(s, \pi(s), s^{\prime}\right) \cdot V^{\pi}\left(s^{\prime}\right)
$$


Obtenção de políticas ótimas. Os métodos para obtenção de políticas ótimas que apresentamos a seguir são baseados na seguinte definição:

Definição 6.2. Sejam $\mathcal{M}$ um MDP $e \Pi$ o conjunto de todas as políticas para $\mathcal{M}$. Dizemos que uma política $\pi^{*}$ é ótima se, para toda política $\pi \in \Pi$ e todo estado $s \in \mathcal{S}$, temos $V^{\pi^{*}}(s) \geq V^{\pi}(s)$; ou seja, uma política é ótima se maximiza a função valor $V$.

Seja $V^{*}$ a função valor de uma política ótima. Uma política gulosa com relação a $V^{*}$, denotada por $\pi^{*}$, é uma política ótima [Puterman, 1994]. Essa política é definida por:

$$
\pi^{*}(s)=\arg \max _{a \in \mathcal{A}}\left\{\mathcal{R}(s)+\gamma \sum_{s^{\prime} \in \mathcal{S}} \mathcal{T}\left(s, a, s^{\prime}\right) \cdot V\left(s^{\prime}\right)\right\}
$$

Os dois métodos básicos para obtenção de políticas ótimas são ${ }^{1}$ :

- Iteração de valor: Esse método calcula o valor $V^{*}$ de uma política ótima e, gradativamente, constrói uma política ótima $\pi^{*}$. Em cada iteração $n$ do processo, consideramos que restam apenas $n$ passos até o final da vida do agente e escolhemos uma ação que maximiza sua recompensa descontada total esperada nesse ponto. À medida em que essas ações vão sendo escolhidas, uma política ótima vai sendo construída. No caso de horizonte finito $(\gamma=1)$, as ações escolhidas em cada iteração $n$ formam uma política não-estacionária ótima $\pi_{n}^{*}$. Já no caso de horizonte infinito $(0<\gamma<1)$, após um número finito de iterações $k$, o processo converge para a função $V^{*}$ [Puterman, 1994]. Então, as ações escolhidas na $k$-ésima etapa desse processo constituem uma política estacionária ótima.

Em cada iteração $n$, a utilidade de executar uma ação $a$ num estado $s$, é definida por:

$$
Q_{n}(s, a)=\mathcal{R}(s)+\gamma \sum_{s^{\prime} \in \mathcal{S}} \mathcal{T}\left(s, a, s^{\prime}\right) \cdot V_{n-1}\left(s^{\prime}\right), \quad \text { para } n>0
$$

A utilidade de um estado $s$ é definida por:

$$
V_{n}(s)= \begin{cases}\mathcal{R}(s) & \text { se } n=0 \\ \max _{a \in \mathcal{A}}\left\{Q_{n}(s, a)\right\} & \text { se } n>0\end{cases}
$$

Finalmente, a ação $\pi_{n}(s)$, que maximiza $V_{n}(s)$, é definida por:

\footnotetext{
${ }^{1}$ Um implementação desses métodos em JAVA é apresentada em [Pereira \& Barros, 2007e].
} 


$$
\pi_{n}(s)=\arg \max _{a \in \mathcal{A}}\left\{Q_{n}(s, a)\right\}, \quad \text { para } n>0
$$

O algoritmo Vi (VAlue Iteration) [Puterman, 1994], apresentado a seguir, recebe como entrada um MDP $\mathcal{M}$ e devolve como saída uma política estacionária ótima $\pi^{*}$.

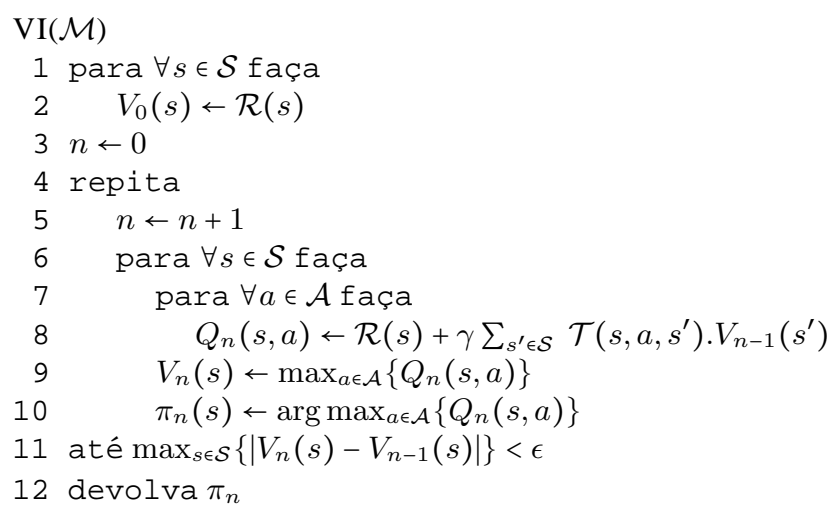

- Iteração de política: Esse método, implementado pelo algoritmo PI (POLICy ItERATION) [Puterman, 1994] apresentado a seguir, começa com uma política inicial aleatória e, a cada iteração, altera essa política de modo que seu valor seja aumentado. Como o número de políticas possíveis é finito, após um número finito de iterações, o valor da política converge para o valor ótimo [Puterman, 1994]. A política obtida na última iteração é, portanto, uma política estacionária ótima. Note que, ao contrário do método de iteração de valor, que pode ser usado para obtenção de políticas não-estacionárias ótimas, o método de iteração de política só permite a obtenção de políticas estacionárias.

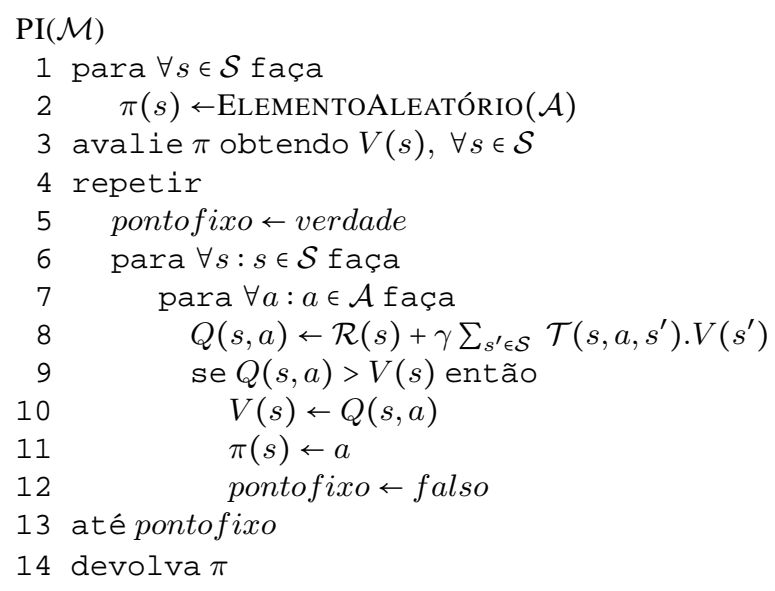




\subsection{Planejamento quantitativo baseado em MDPs}

Nessa seção, mostramos como o arcabouço de MDPs pode ser usado para implementar planejamento quantitativo (também denominado planejamento probabilístico).

\subsubsection{Domínios de planejamento}

Sejam $\mathbb{P} \neq \varnothing$ um conjunto finito de proposições atômicas, que denotam propriedades de estados do ambiente de planejamento, e $\mathbb{A} \neq \varnothing$ um conjunto finito de ações, que representam as habilidades do agente nesse ambiente.

Definição 6.3. Um domínio de planejamento quantitativo com assinatura $(\mathbb{P}, \mathbb{A})$ é definido por uma tupla da forma $\mathcal{D}=\langle\mathcal{S}, \mathcal{L}, \mathcal{T}\rangle$, onde:

- $\mathcal{S} \neq \varnothing$ é um conjunto finito de estados do ambiente;

- $\mathcal{L}: \mathcal{S} \mapsto 2^{\mathbb{P}}$ é uma função de rotulação de estados;

- $\mathcal{T}: \mathcal{S} \times \mathbb{A} \times \mathcal{S} \mapsto[0,1]$ é uma função de transição de estados probabilística.

Dados dois estados $s, s^{\prime} \in \mathcal{S}$ e uma ação $a \in \mathbb{A}$, a probabilidade de alcançar o estado $s^{\prime}$, dado que a ação $a$ foi executada no estado $s$, é denotada por $\mathcal{T}\left(s, a, s^{\prime}\right)$. Ademais, para todo estado $s \in \mathcal{S}$, se existem uma ação $a \in \mathbb{A}$ e um estado $s^{\prime} \in \mathcal{S}$ tal que $\mathcal{T}\left(s, a, s^{\prime}\right) \neq 0$, então:

$$
\sum_{s^{\prime} \in \mathcal{S}} \mathcal{T}\left(s, a, s^{\prime}\right)=1
$$

Também assumimos que o conjunto de ações $\mathbb{A}$ contém a ação trivial $\tau$, cuja execução mantém o estado corrente do ambiente inalterado. Particularmente para essa ação, para todo $s, s^{\prime} \in \mathcal{S}$, temos que:

$$
\mathcal{T}\left(s, \tau, s^{\prime}\right)=\left\{\begin{array}{lll}
0 & \text { se } & s \neq s^{\prime} \\
1 & \text { se } & s=s^{\prime}
\end{array}\right.
$$

Assim, quando o agente executa a ação $\tau$ em um determinado estado, ele sempre permanece nesse mesmo estado. Intuitivamente, a ação $\tau$ representa o fato que, em qualquer estado, o agente sempre pode escolher não fazer nada. 
Dado um estado $s \in \mathcal{S}$, o conjunto de ações executáveis em $s$, denotado por $\mathbb{A}(s)$, é o conjunto $\left\{a \in \mathbb{A}: \exists s^{\prime} . \mathcal{T}\left(s, a, s^{\prime}\right)>0\right\}$. Assumimos que, para todo estado $s \in \mathcal{S}, \tau \in \mathbb{A}(s)$. Dizemos que um estado $s \in \mathcal{S}$ é terminal se $\mathbb{A}(s)=\{\tau\}$.

\subsubsection{Problemas e soluções}

Como definimos Seção 6.1, a função de recompensa $\mathcal{R}$ é uma componente importante na especificação de um MDP. Intuitivamente, quando o agente visita um estado $s$, como recompensa, ele recebe uma quantia $\mathcal{R}(s)$. Assim, estados com recompensas muito baixas funcionam como pólos repelentes; enquanto estados com recompensas muito altas funcionam como pólos atrativos para $o$ agente.

Em termos de planejamento, podemos definir um estado meta num MDP como sendo um estado que tem recompensa máxima (i.e., um estado absorvente). Isso significa que, para cada nova meta de planejamento, uma nova função de recompensa deve ser especialmente formulada. Por esse motivo, funções de recompensa não podem ser fornecidas como parte integrante da especificação de um domínio de planejamento (que deve ser suficientemente genérica para permitir que o agente possa planejar para diferentes metas, em diferentes momentos). Funções de recompensa constituem uma linguagem muito expressiva, que permite especificar metas bastante complexas [Ghallab et al., 2004]. Apesar disso, entretanto, funções de recompensa são extremamente dependentes do problema e, freqüentemente, são definidas de maneira ad hoc.

Dado um problema de planejamento quantitativo $\mathcal{P}$, especificado por um domínio $\mathcal{D}$ e por uma função de recompensa $\mathcal{R}$, uma solução para $\mathcal{P}$ é uma política ótima $\pi$, que associa a cada estado $s \in \mathcal{S}$ uma ação $a \in \mathbb{A}$ que produz o valor máximo esperado. Tal política $\pi$ pode ser obtida diretamente pelo método de iteração de valor (algoritmo VI) apresentado na Subseção 6.1.3.

\subsubsection{Qualidade das soluções}

Nessa subseção, mostramos como podemos obter soluções de diferentes qualidades para problemas de planejamento quantitativo para metas de alcançabilidade simples.

Planejamento para metas de alcançabilidade simples. Dada uma fórmula proposicional $\phi$ sobre $\mathbb{P}$, especificando uma meta de alcançabilidade simples, uma função de recompensa corres- 
pondente pode ser definida conforme a seguir:

$$
\mathcal{R}(s)=\left\{\begin{array}{ccc}
0 & \text { se } & s \not \phi \\
1 & \text { se } & s \vDash \phi
\end{array}\right.
$$

Dado um problema de planejamento $\mathcal{P}$, especificado por um domínio quantitativo $\mathcal{D}$ e por uma meta de alcançabilidade simples $\phi$, uma solução para $\mathcal{P}$ pode ser obtida pelo algoritmo de iteração de valor VI, adaptado da seguinte forma:

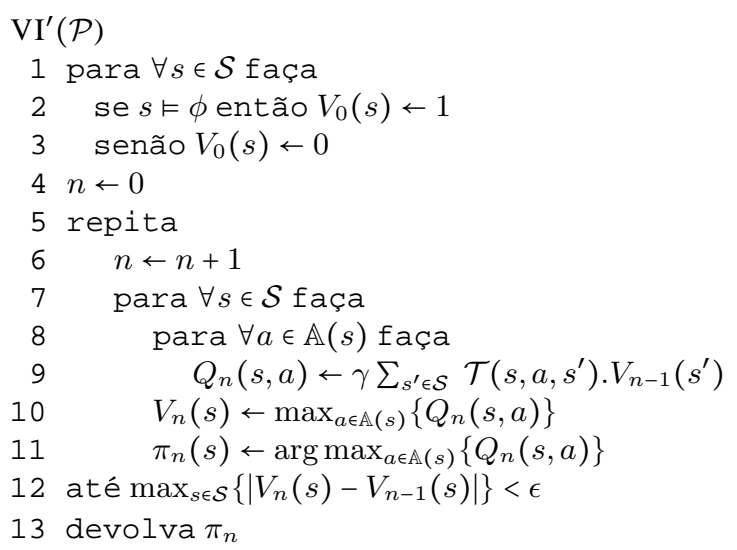

Esse algoritmo inicia atribuindo recompensa 1 aos estados que satisfazem a meta especificada pela fórmula $\phi$ (e 0 aos demais), conforme definido pela Equação 6.13. Intuitivamente, essas recompensas servem como um "incentivo" que atrai o agente para os estados metas. Em seguida, iterativamente, o algoritmo refina os valores dos estados, selecionando uma ação que maximize a recompensa esperada em cada um deles. A cada iteração $n$, e para cada estado $s$, o valor $V_{n}(s)$ é atualizado com base nos valores $V_{n-1}($.$) , que foram computados na iteração anterior. Para forçar$ o agente a preferir caminhos mais curtos para alcançar estados metas, a cada ação executada (i.e., transição de $s$ para $s^{\prime}$ ), recompensas futuras são descontadas por um fator $0<\gamma<1$. Portanto, se o agente alcança um estado meta através de um caminho de comprimento $n$ (i.e., após executar $n$ ações), ele recebe uma recompensa de $\gamma^{n}$. Assim, para maximizar a sua recompensa, o agente deve minimizar o comprimento dos caminhos para os estados metas.

Segundo [Ghallab et al., 2004], pode ser demonstrado que existe um número máximo de iterações necessárias para garantir que o algoritmo $\mathrm{VI}^{\prime}$ termina e devolve uma política ótima para o problema $\mathcal{P}$. Entretanto, em aplicações práticas, a condição normalmente utilizada para terminar o processo de iteração é a seguinte: 


$$
\max _{s \in \mathcal{S}}\left|V_{n}(s)-V_{n-1}(s)\right|<\epsilon .
$$

Com essa condição, podemos garantir que o algoritmo termina e devolve uma política $\epsilon$-ótima (i.e., para cada estado $s \in \mathcal{S}$, o valor $V^{\pi}(s)$ não difere do valor ótimo $V^{\pi^{\star}}(s)$ por mais que um erro constante $\epsilon$, arbitrariamente pequeno).

Soluções fracas. A seguir, apresentamos dois exemplos bastante simples que mostram que o algoritmo VI' (baseado num método clássico de solução de MDPs) não garante soluções fortes, nem fortes-cíclicas ${ }^{2}$. De fato, a única garantia que esse algoritmo oferece é que será devolvida uma política que permite ao agente um desempenho ótimo no caso esperado.

O Exemplo 6.1 mostra que, mesmo existindo uma solução forte, o algoritmo VI' pode devolver uma solução forte-cíclica.

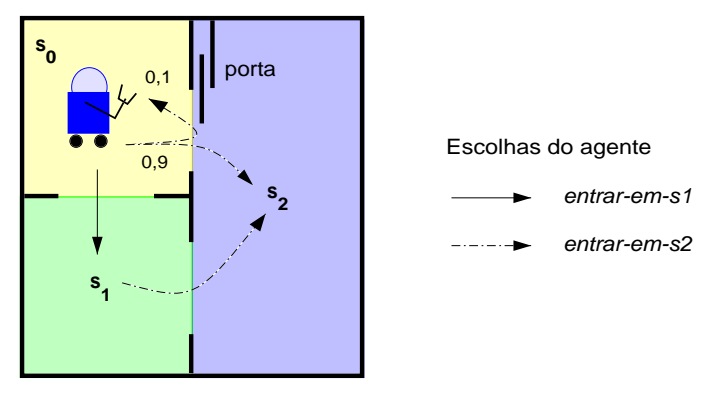

Figura 6.2: Domínio em que a solução ótima é forte-cíclica.

Exemplo 6.1 (O algoritmo VI' não garante solução forte). Considere o domínio de planejamento quantitativo ilustrado na Figura 6.2. Nesse domínio, ao executar a ação entrar-em- $s_{2}$, o agente pode acabar batendo na porta automática e permanecendo na sala $s_{0}$. Suponha que a meta do agente nesse domínio seja chegar à sala $s_{2}$. Apesar de existir uma solução forte para esse problema, o algoritmo $\mathrm{VI}^{\prime}$ devolve a seguinte solução forte-cíclica:

- em $s_{0}$, execute a ação entrar-em-s $s_{2}$;

- em $s_{1}$, execute a ação entrar-em- $s_{2}$;

- em $s_{2}$, execute a ação esperar.

\footnotetext{
${ }^{2}$ As soluções dos exemplos foram geradas pelo programa implementado em [Pereira \& Barros, 2007e].
} 
O Exemplo 6.2 mostra que, mesmo existindo uma solução forte-cíclica, o algoritmo VI' pode devolver uma solução estritamente fraca.

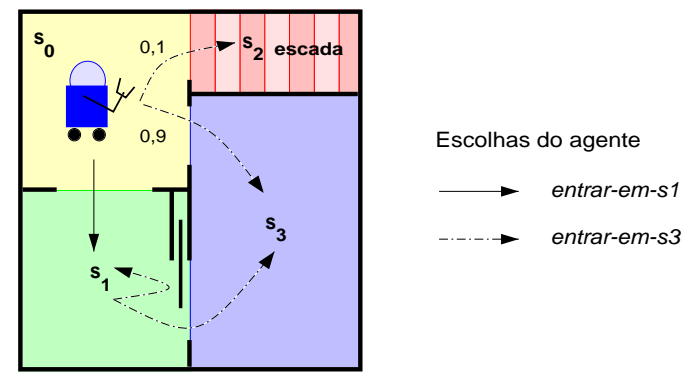

Figura 6.3: Domínio em que a solução ótima é estritamente fraca.

Exemplo 6.2 (O algoritmo VI' não garante solução forte-cíclica). Considere o domínio de planejamento quantitativo ilustrado na Figura 6.3. Nesse domínio, quando o agente tenta entrar-em$s_{3}$, a partir da sala $s_{0}$, ele pode acabar entrando na sala $s_{2}$, onde há uma escada que o impede de sair. Além disso, quanto o agente tenta entrar-em- $s_{3}$, a partir da sala $s_{1}$, ele pode bater na porta automática e permanecer no mesmo lugar. Suponha que a meta do agente nesse domínio seja chegar à sala $s_{3}$. Apesar de existir uma solução forte-cíclica para esse problema, o algoritmo VI' devolve a seguinte solução estritamente fraca:

- em $s_{0}$, execute a ação entrar-em-s $s_{3}$

- em $s_{1}$, execute a ação entrar-em-s $s_{3}$;

- em $s_{2}$, execute a ação esperar;

- em $s_{3}$, execute a ação esperar.

Como podemos constatar, o algoritmo VI' é um algoritmo de planejamento quantitativo fraco. Embora soluções fracas possam ser aceitáveis em algumas aplicações de planejamento automatizado, há muitas situações práticas em que falhas não são toleradas. Nessas situações, uma política que possa resultar em caminhos de execução mais longos, mas que necessariamente alcance um estado meta, é preferível a uma política que num caso otimista alcança um estado meta rapidamente e num caso pessimista fracassa em alcançar a meta de planejamento.

Soluções fortes-cíclicas. Quando soluções fortes-cíclicas são necessárias, um problema de planejamento quantitativo para meta de alcançabilidade simples pode ser visto como uma instân- 
cia do problema denominado caminho estocástico mínimo (SSP - STOCHASTIC SHORTEST PATH)

[Bertsekas \& Tsitsiklis, 1991]. Esse problema é formulado com base nas seguintes suposições:

- existe um estado inicial bem definido $s_{0} \in \mathcal{S}$;

- um estado meta pode ser alcançado com probabilidade 1 , a partir de qualquer estado $s \in \mathcal{S}$;

- a solução é uma política ótima parcial $\pi^{\star}: \mathcal{S} \mapsto \mathbb{A}$.

Ademais, caso existam várias políticas ótimas $\pi_{1}^{\star}, \ldots, \pi_{n}^{\star}$, aquela que cobre o menor subconjunto de $\mathcal{S}$ é considerada preferível. Note que essas suposições não são feitas nos MDPs mais gerais.

Dois algoritmos para solução de SSPs bastante conhecidos são:

- RtdP (Real-Time Dynamic Programming) [Barto et al., 1993] é uma versão baseada em simulação do algoritmo de iteração de valor [Puterman, 1994]. Essencialmente, RTDP é um método guloso que, partindo do estado inicial $s_{0}$, percorre vários caminhos até um estado meta. À medida em que esses caminhos vão sendo percorridos, para cada estado $s \in \mathcal{S}$ visitado, o algoritmo escolhe a ação $a \in \mathbb{A}$ que maximiza o valor da função $Q(s, a)$. A terminação das simulações é garantida pela hipótese de que um estado meta pode ser alcançado com probabilidade 1 , a partir do estado inicial $s_{0}$. Ademais, para guiar as simulações, o algoritmo utiliza também uma função de avaliação heurística $H$, que faz com que os caminhos mais prováveis sejam percorridos com maior freqüência. A cada iteração do algoritmo, a função RTDPTRIAL é chamada para encontrar a ação gulosa com relação a $H$ no estado corrente $s$. Ao final de cada chamada, os valores da heurística $H(s)$ e da ação $\pi(s)$ são atualizados. Caso a heurística fornecida inicialmente seja admissível, então o algoritmo RTDP a fará convergir para a função valor ótima $V^{\star}$ e, conseqüentemente, a política $\pi$ também convergirá para uma política ótima $\pi^{\star}$.

- LrTdP (Labeled Real-Time Dynamic Programming) [Bonet \& Geffner, 2003b] é uma versão do algoritmo RTDP que rotula estados para os quais a função valor já convergiu. Devido à estratégia de exploração do espaço de estados adotada pelo algoritmo RTDP, caminhos improváveis tendem a ser ignorados, o que torna a convergência do método bastante lenta. Com o mecanismo de rotulação introduzido nessa nova versão, entretanto, quando os estados nos caminhos mais prováveis já convergiram (i.e., já foram rotulados como "resolvidos"), o algoritmo é forçado a escolher caminhos menos prováveis, fazendo com que a velocidade de convergência do método seja acelerada. 


\subsection{Planejamento probabilístico forte}

Como mostramos na seção anterior, existem algoritmos que garantem encontrar soluções fracas (e.g., VI [Puterman, 1994]) e fortes-cíclicas (e.g., RTDP [Bertsekas \& Tsitsiklis, 1991]) para problemas de planejamento quantitativo. Entretanto, até onde sabemos ${ }^{3}$, não existem algoritmos que garantam encontrar soluções fortes para esse tipo de problema. Nessa seção, propomos um algoritmo de planejamento probabilístico forte [Pereira et al., 2007], que combina técnicas de verificação de modelos (vide Capítulo 2) e teoria da decisão (vide Apêndice C). Nesse algoritmo, a função de pré-imagem forte é utilizada para garantir que apenas soluções fortes sejam consideradas durante o planejamento; enquanto o princípio de otimalidade [Bellman, 1957] é utilizado para garantir que apenas uma política ótima, entre aquelas consideradas durante o planejamento, seja devolvida como solução final.

\subsubsection{Necessidade de soluções fortes}

Muitas aplicações de planejamento quantitativo envolvem tarefas de alto-risco, que podem resultar em grandes perdas de dinheiro, equipamentos ou, até mesmo, vidas humanas. Como exemplos dessas aplicações, podemos citar controle de veículos [Simmons et al., 1994, Pell et al., 1998, Zilberstein et al., 2002], agentes de negócios [Goodwin et al., 2002], controle de desastres ambientais [Cohen et al., 1989] e planejamento de processos industriais [Murthy et al., 1999]. Nessas aplicações, maximizar recompensas apenas no caso esperado nem sempre é a escolha mais adequada [Liu \& Koenig, 2005]. O Exemplo 6.3, adaptado de [Taha, 1987]), ilustra essa idéia.

Exemplo 6.3 (Aversão a riscos). Considere as seguintes opções: (i) inverstir uma determinada quantia num negócio que pode resultar num ganho de $R$ \$10.000,00 ou nada, com igual probabilidade; ou (ii) investir a mesma quantia num outro negócio com ganho garantido de $R \$ 4.000,00$. Muitos investidores certamente prefeririam a opção (ii), apesar do ganho esperado ser menor. Tais investidores são aversos a riscos, ou seja, eles preferem a segurança de um bom desempenho no pior caso. Assim, um agente que escolhesse maximizar o ganho apenas no caso esperado (i.e., opção (i)), em metade das vezes, deixaria muitos investidores bastante descontentes.

\footnotetext{
${ }^{3}$ O algoritmo de busca $\mathrm{AO}^{\star}$ [Martelli \& Montanari, 1973] garante encontrar soluções fortes, mas requer o espaço de busca seja acíclico (uma restrição que não desejamos impor aos domínios de planejamento que estamos considerando).
} 
A despeito da incerteza associada aos efeitos das ações, em muitas aplicações práticas:

- falhas não são toleradas - é preciso garantir que, executando a política sintetizada pelo planejador, o agente alcançará a sua meta sem correr riscos. Por exemplo, numa aplicação envolvendo um agente móvel, não requemos correr o risco de que esse agente seja danificado durante a execução de uma tarefa, ainda que esse risco seja muito pequeno;

- tempo é um recurso limitado - é preciso garantir que a meta será alcançada o mais rapidamente possível no caso esperado, dentro de um período de tempo limitado e mínimo no pior caso. Por exemplo, se um agente móvel tem uma bateria com carga limitada, não queremos correr o risco de que a execução do plano se estenda além da quantidade de energia disponível nessa bateria.

Com o algoritmo de planejamento probabilístico que propomos nessa seção, garantimos a síntese de soluções robustas, com restrições de qualidade que vão além daquelas que a teoria usual de MDPs ou as técnicas de verificação de modelos podem garantir isoladamente. Mais especificamente, garantimos a síntese de políticas acíclicas (que executam em tempo limitado, mesmo em domínios cíclicos) que alcançam a meta de planejamento com 100\% de probabilidade, executando o mínimo de passos no pior caso, com desempenho ótimo no caso esperado.

Devemos ainda salientar que, em princípio, o problema de planejamento probabilístico forte que tratamos é um tipo de MDP restrito [Altman, 1999]; ou seja, um MDP com restrições adicionais sobre trajetórias. Há, porém, duas dificuldades em se empregar diretamente a teoria geral de MDPs restritos: a primeira delas é que não é inteiramente óbvio como podemos modelar a restrição "probabilidade zero de que um ciclo ocorra", de uma forma que permita a busca eficiente de uma solução; a segunda é que, para MDPs restritos, o princípio de programação dinâmica [Bellman, 1957] não pode ser aplicado e, portanto, eles não podem ser tratados pelos algoritmos normalmente empregados para resolver MDPs. De fato, soluções para MDPs restritos, em geral, são políticas aleatorizadas, que requerem o uso de programação linear para sua obtenção. Por outro lado, o método de planejamento probabilístico forte que propomos admite indução regressiva e, portanto, é mais simples e mais eficiente que a abordagem mais geral baseada em MDPs restritos [Dolgov \& Durfee, 2005].

\subsubsection{Domínio quantitativo $v s$. qualitativo}

Para apresentarmos o algoritmo híbrido que propomos, precisamos antes definir de que modo um domínio de planejamento quantitativo pode ser interpretado como qualitativo. De acordo com as 
Definições 3.2 e 6.3, a única diferença entre esses dois tipos de domínios de planejamento está no tipo de função de transição de estados considerada em cada um deles; sendo que nos domínios quantitativos essa função é probabilística. Assim, para interpretar um domínio quantitativo como qualitativo, basta definir a função de transição de estados do modelo qualitativo em termos da função de transição de estados do modelo quantitativo.

Dada a função de transição $\mathcal{T}: \mathcal{S} \times \mathbb{A} \times \mathcal{S} \mapsto[0,1]$ de um domínio quantitativo, a função de transição $\mathcal{T}: \mathcal{S} \times \mathbb{A} \mapsto 2^{\mathcal{S}}$, do domínio qualitativo correspondente, é definida como:

$$
\mathcal{T}(s, a)=\left\{s^{\prime} \in \mathcal{S}: \mathcal{T}\left(s, a, s^{\prime}\right)>0\right\}
$$

\subsubsection{Metas de alcançabilidade simples}

Dado um problema de planejamento quantitativo para meta de alcançabilidade simples $\mathcal{P}=$ $\left\langle\mathcal{D}, s_{0}, \phi\right\rangle$, o algoritmo de planejamento probabilístico forte PPF [Pereira et al., 2007] inicia atribuindo recompensa 1 aos estados metas (i.e., estados que satisfazem a fórmula $\phi$ ) e construindo uma política $\pi$ que associa, a cada um desses estados, a ação trivial $\tau$. Em seguida, alternadamente, o algoritmo PPF computa pré-imagens fortes e valores ótimos esperados. Usando a função PRÉIMAGEMForTEMAP, ele garante que a política sintetizada necessariamente alcançará a meta de planejamento (independentemente do não-determinismo); e usando a função EsCOLHA, ele garante que sempre que um estado for mapeado a mais que uma ação (pela função de pré-imagem forte), apenas uma ação com valor esperado ótimo entre elas poderá ser escolhida naquele estado.

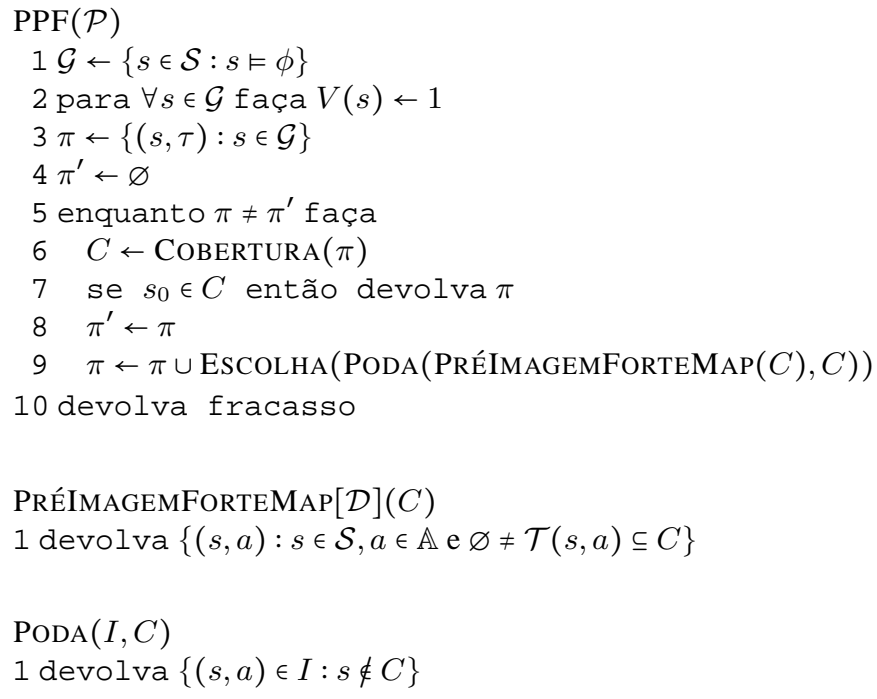


Conforme podemos ver a seguir, a função EsCOLHA implementa o princípio de otimalidade [Bellman, 1957] usando indução regressiva. Isso é possível porque o uso combinado das funções PRÉIMAGEMForTEMAP e PODA evita ciclos, permitindo que os estados cobertos pela política em construção possam ser ordenados topologicamente (Figura 6.5). Assim, processando os estados em ordem topológica reversa, quando o valor de um estado é computado, o valor de cada um dos seus possíveis sucessores já é conhecido.
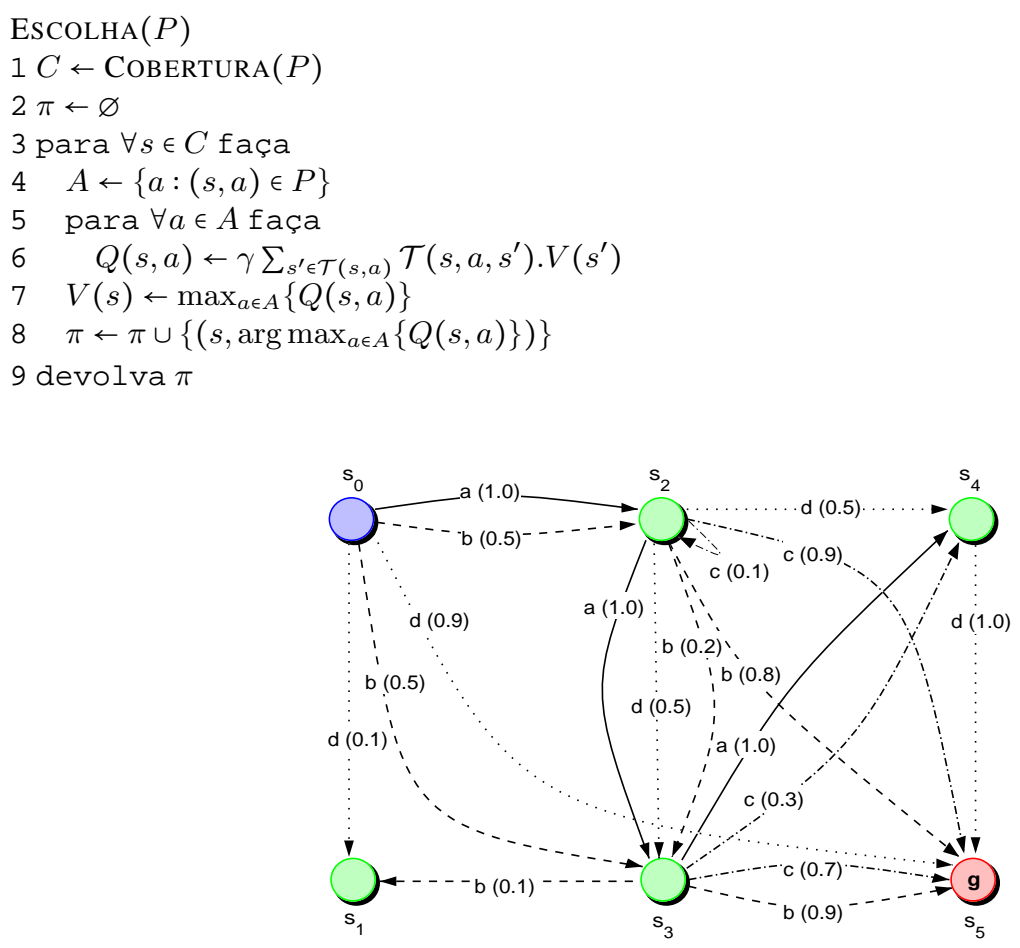

Figura 6.4: Um domínio de planejamento probabilístico.

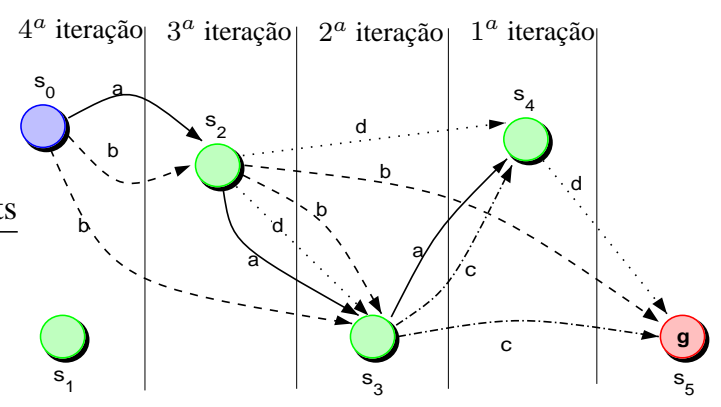

Figura 6.5: Ordenação topológica dos estados do domínio na Figura 6.4. 


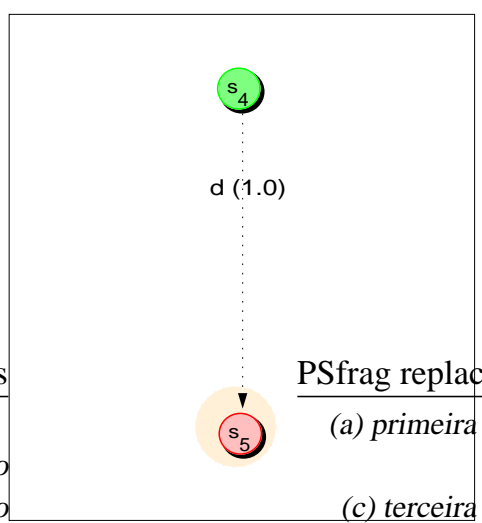

(a) primeira iteração

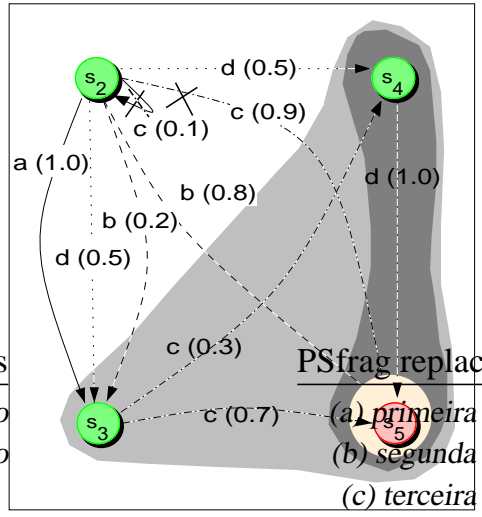

(c) terceira iteração

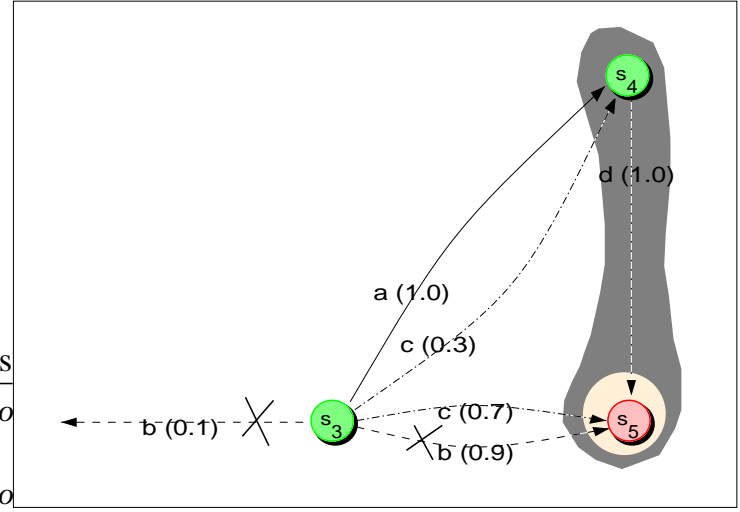

(b) segunda iteração

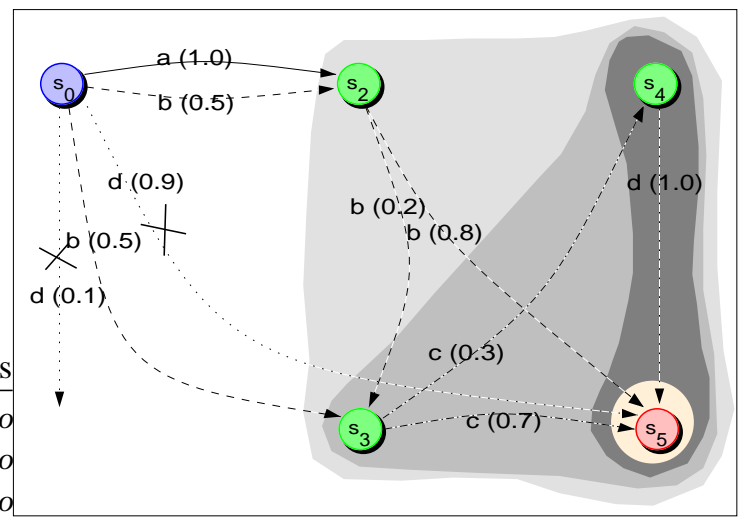

(d) quarta iteração

Figura 6.6: Execução do algoritmo de planejamento probabilístico forte.

O Exemplo 6.4 fornece uma intuição sobre o funcionamento do algoritmo PPF.

Exemplo 6.4 (Funcionamento do algoritmo PPF). Considere o problema de planejamento $\mathcal{P}=$ $\left.\left\langle\mathcal{D}, s_{0}, g\right\}\right\rangle$, onde $\mathcal{D}$ é o domínio ilustrado na Figura 6.4. Inicialmente, temos $V\left(s_{5}\right)=1$ e $\pi=\left\{\left(s_{5}, \tau\right)\right\}$. Então, para $\gamma=0.9$ :

- Na primeira iteração (Figura 6.6-a), a pré-imagem forte podada da cobertura da política $\pi$ é o conjunto $\left\{\left(s_{4}, d\right)\right\}$. Calculando o valor esperado da ação d no estado $s_{4}$, temos $Q\left(s_{4}, d\right)=\gamma \times 1.0 \times V\left(s_{5}\right)=0.9$. Assim, fazemos $V\left(s_{4}\right)=0.9$ e $\pi=\left\{\left(s_{4}, d\right),\left(s_{5}, \tau\right)\right\}$.

- Na segunda iteração (Figura 6.6-b), a pré-imagem forte podada da cobertura da política $\pi$ 
é o conjunto $\left\{\left(s_{3}, a\right),\left(s_{3}, c\right)\right\}$. Usando pré-imagem forte, evitamos a ação $b$, que poderia causar uma falha caso fosse executada no estado $s_{3}$ (pois a execução de $b$ em $s_{3}$ pode levar o agente ao estado terminal $s_{1}$ ). Os valores esperados para as demais ações são:

$Q\left(s_{3}, a\right)=\gamma \times 1.0 \times V\left(s_{4}\right)=0.81$

$Q\left(s_{3}, c\right)=\gamma \times\left(0.3 \times V\left(s_{4}\right)+0.7 \times V\left(s_{5}\right)\right)=0.87$

Com base nesses valores, claramente, devemos preferir a ação c. Então, fazemos $V\left(s_{3}\right)=$ 0.87 e $\pi=\left\{\left(s_{3}, c\right),\left(s_{4}, d\right),\left(s_{5}, \tau\right)\right\}$.

- Na terceira iteração (Figura 6.6-c), a pré-imagem forte podada da cobertura da política $\pi$ é o conjunto $\left\{\left(s_{2}, a\right),\left(s_{2}, b\right),\left(s_{2}, d\right)\right\}$. Nesse caso, a computação da pré-imagem forte descarta a ação c, cuja execução em $s_{2}$ poderia causar um ciclo. Os valores esperados para as demais ações são:

$Q\left(s_{2}, a\right)=\gamma \times\left(1.0 \times V\left(s_{3}\right)\right)=0.79$

$Q\left(s_{2}, b\right)=\gamma \times\left(0.2 \times V\left(s_{3}\right)+0.8 \times V\left(s_{5}\right)\right)=0.88$

$Q\left(s_{2}, d\right)=\gamma \times\left(0.5 \times V\left(s_{3}\right)+0.5 \times V\left(s_{4}\right)\right)=0.80$

Sendo a ação b a melhor escolha possível para o estado $s_{2}$. Então, fazemos $V\left(s_{2}\right)=0.88$ e $\pi=\left\{\left(s_{2}, b\right),\left(s_{3}, c\right),\left(s_{4}, d\right),\left(s_{5}, \tau\right)\right\}$

- Finalmente, na última iteração (Figura 6.6-d), a pré-imagem forte podada da cobertura da política $\pi$ é o conjunto $\left\{\left(s_{0}, a\right),\left(s_{0}, b\right)\right\}$. A ação d, que poderia causar uma falha, é descartada. Os valores esperados são:

$$
\begin{aligned}
& Q\left(s_{0}, a\right)=\gamma \times\left(1.0 \times V\left(s_{2}\right)\right)=0.789 \\
& Q\left(s_{0}, b\right)=\gamma \times\left(0.5 \times V\left(s_{3}\right)+0.5 \times V\left(s_{2}\right)\right)=0.787
\end{aligned}
$$

Agora, a melhor escolha é a ação a. Então, fazemos $V\left(s_{0}\right)=0.789$ e atualizamos a política em construção para $\pi=\left\{\left(s_{0}, a\right),\left(s_{2}, b\right),\left(s_{3}, c\right),\left(s_{4}, d\right),\left(s_{5}, \tau\right)\right\}$. Como o estado inicial $s_{0}$ é coberto por essa política, o algoritmo de planejamento probabilístico forte pára e devolve a política $\pi$ como solução.

Os Teoremas 6.1 e 6.2, cujas provas são apresentadas no Apêndice A, garantem a otimalidade da solução devolvida pelo algoritmo PPF. 
Teorema 6.1. Se um problema de planejamento quantitativo $\mathcal{P}$ tem uma solução forte, então o algoritmo $\operatorname{PPF}(\mathcal{P})$ devolve uma política para $\mathcal{P}$ que é ótima no pior caso.

Teorema 6.2. A política devolvida pelo algoritmo $\operatorname{PPF}(\mathcal{P})$ é uma ótima no caso esperado, entre todas aquelas que são fortes e ótimas no pior caso.

\subsubsection{Metas de alcançabilidade estendidas}

Conforme a Definição 5.1, uma meta de alcançabilidade estendida é um par de fórmulas $\left(\varphi_{1}, \varphi_{2}\right)$, onde $\varphi_{1}$ especifica uma condição a ser preservada durante a execução de uma política e $\varphi_{2}$ especifica uma condição a ser alcançada ao final da execução dessa política.

Para que o algoritmo PPF possa resolver problemas com metas de alcançabilidade estendidas, bastam pequenas modificações. A principal dessas modificações ocorre na função de poda, que agora também recebe como parâmetro o conjunto $I_{1}$, contendo todos os estados que satisfazem a condição $\varphi_{1}$, a ser preservada ao longo dos caminhos de execução da política sendo construída.

$\operatorname{PoDA}^{\prime}\left(R, S, I_{1}\right)$

1 devolva $\left\{(s, a) \in R: s \notin S\right.$ e $\left.s \in I_{1}\right\}$

Nessa nova versão, além dos pares $(s, a)$ cujos estados já foram cobertos em alguma das iterações anteriores do algoritmo de planejamento probabilístico, a função PODA' deve descartar também aqueles pares cujos estados não satisfazem a condição de preservação $\varphi_{1}$.

Algumas modificação simples também são necessárias em PPF:

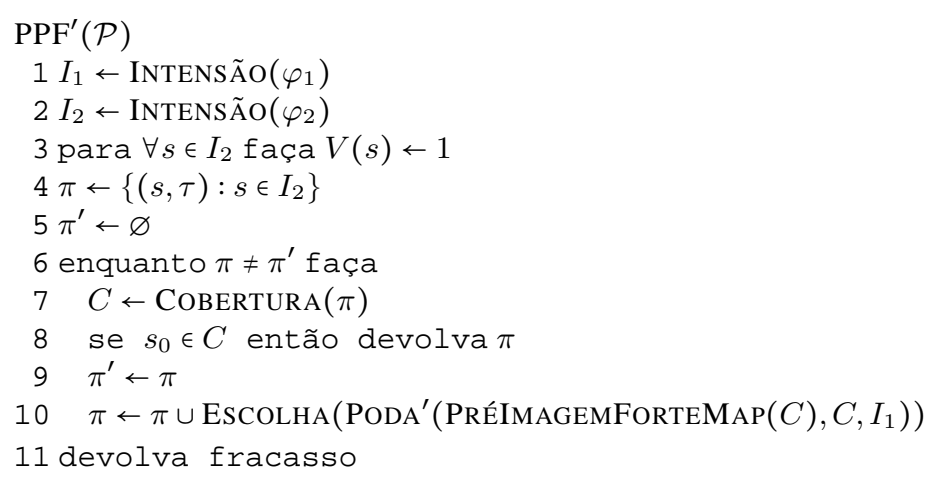

Essa nova versão do algoritmo PPF, utiliza a função InTEnsão, apresentada na Subseção 5.3.3, para computar os conjuntos de estados que satisfazem as fórmulas $\varphi_{1}$ e $\varphi_{2}$, especificadas 
em $\alpha$-CTL. As funções PréImagemForteMap e Escolha, apresentadas na Subseção 6.3.3, não precisam de nenhuma alteração para serem utilizadas pelo algoritmo $\mathrm{PPF}^{\prime}$.

\subsection{Sumário}

Nesse capítulo, apresentamos o arcabouço de planejamento quantitativo baseado em processos de decisão markovianos [Boutilier et al., 1999, Pereira \& Barros, 2007e] e discutimos a qualidade das soluções obtidas com esse arcabouço. Particularmente, notamos que existem algoritmos capazes de encontrar soluções fracas (e.g., VI [Puterman, 1994]) e fortes-cíclicas (e.g., RTDP [Bertsekas \& Tsitsiklis, 1991]) para problemas de planejamento quantitativo; mas não existem algoritmos capazes de garantir soluções fortes para esse tipo de problema. Motivados por essa constatação, apresentamos um algoritmo de planejamento probabilístico forte [Pereira et al., 2007], que combina técnicas de verificação de modelos e teoria da decisão, para resolver problemas de planejamento quantitativo para metas de alcançabilidade simples. Finalmente, concluímos o capítulo mostrando como o algoritmo híbrido proposto pode ser modificado para tratar também metas de alcançabilidade estendidas. 


\section{Capítulo 7}

\section{Conclusão}

Nos últimos anos, planejamento automatizado vem sendo cada vez mais requisitado em aplicações práticas de diversas áreas que requerem soluções confiáveis para problemas de planejamento envolvendo metas complexas. Esses problemas, em geral, além de possuírem enormes espaços de estados, ainda dependem de tomada de decisão sob incerteza, o que dificulta não apenas a especificação de metas com requisitos de qualidade desejados, mas também a síntese e a validação de planos para alcançar essas metas. Em decorrência desse fato, várias abordagens interessantes foram propostas para tratar problemas de planejamento sob incerteza. Entre elas, verificação de modelos tem se mostrado uma opção muito atrativa, sobretudo, por dois motivos:

- permite a implementação de métodos de planejamento eficientes e altamente escaláveis;

- é baseada no uso de métodos formais, o que torna mais confiáveis as soluções obtidas pelos métodos de planejamento.

Também observamos que mesmo os planejadores que representam o estado-da-arte na área de planejamento automatizado ainda estão, na sua maioria, voltados para o tratamento de metas de alcançabilidade simples, como aquelas consideradas no planejamento clássico, que não nos permitem especificar ou resolver problemas de planejamento envolvendo metas mais complexas.

Em vista disso, nessa tese propusemos uma forma de aumentar a expressividade das metas de planejamento clássico, que resultou na classe de metas que denominamos metas de alcançabilidade estendidas. Ademais, para tratar essa classe de metas mais complexas, que até então ainda não havia sido explorada pela comunidade de planejamento, adotamos o arcabouço de verificação de modelos. Um problema que encontramos ao adotar esse arcabouço é que as técnicas existentes 
em verificação de modelos estão quase sempre relacionadas ao uso da lógica temporal CTL, cuja expressividade se mostrou inadequada para tratar a classe de metas de alcançabilidade estendidas que propusemos. Assim, precisamos definir uma nova versão da lógica CTL, que denominamos $\alpha$-CTL. Usando $\alpha$-CTL, pudemos então especificar metas de alcançabilidade estendidas (e respectivas qualidades de soluções desejadas), bem como formalizar algoritmos de síntese e validação de planos para essas metas. Finalmente, discutimos como garantir a qualidade das soluções quando dispomos de um modelo do ambiente de planejamento onde as probabilidades das transições causadas pela execução das ações são conhecidas.

\subsection{Trabalhos correlatos}

Alguns trabalhos correlatos importantes são discutidos a seguir.

Metas estendidas e qualidades de soluções. Conforme mostramos na Seção 5.2, existem metas estendidas que não podemos tratar usando CTL. Motivados por essa mesma observação, Baral \& Zhao propuseram uma nova lógica temporal de tempo ramificado, denominada P-CTL [Baral \& Zhao, 2004, Baral \& Zhao, 2006], que estende a lógica CTL adicionando quantificadores de políticas. Usando P-CTL, além de podermos especificar metas estendidas que não podem ser especificadas em CTL, podemos ainda especificar qualidades mistas de soluções (i.e., critérios que relaxam a restrição de qualidade desejada, em função das limitações impostas pelas características do domínio de planejamento; sendo que a solução obtida pode ser composta de uma parte forte, uma parte forte-cíclica e uma parte fraca, com prioridades decrescentes).

De fato, a classe de metas estendidas que podem ser especificadas em P-CTL é mais abrangente que a classe de metas estendidas que podem ser especificadas em $\alpha$-CTL. Entretanto, até onde sabemos, nenhum algoritmo para síntese de políticas para metas especificadas em P-CTL foi proposto. Em [Baral \& Zhao, 2006], os autores mostram apenas como alguns tipos de metas estendidas podem ser especificados em P-CTL e como a semântica dessa lógica pode ser usada para a validação de políticas dadas para essas metas (essa mesma crítica é feita em [Thiébaux et al., 2006]). Assim, embora $\alpha$-CTL seja menos expressiva que P-CTL, acreditamos que a nossa abordagem é mais prática (já que apresentamos os algoritmos necessários para a síntese de políticas) e também mais eficiente que aquela proposta em [Baral \& Zhao, 2006] (já que a operação de quantificação sobre políticas em P-CTL tem um alto custo computacional). 
Abordagens híbridas. Problemas de planejamento reais freqüentemente são modelados como instâncias do problema do caminho estocástico mínimo (vide Subseção 6.2.3). Para esses problemas, planejamento quantitativo baseado em MDPs tem se mostrado uma abordagem bastante promissora; apesar dos atuais algoritmos para solução de MDPs (e.g., VI [Puterman, 1994] e LRTDP [Bonet \& Geffner, 2003b]) ainda não serem capazes de tratar problemas com espaços de estados realmente grandes. Por outro lado, muitos pesquisadores têm apontado planejamento qualitativo baseado em verificação de modelos como uma opção que pode ser mais atrativa. Planejadores desenvolvidos dentro dessa abordagem (e.g. MBP [Bertoli et al., 2001]) não fazem uso de informações sobre probabilidades e, por resolverem um problema supostamente mais simples, apresentam escalabilidade maior que aquela apresentada pelos planejadores quantitativos.

De fato, Mausam, Bertoli \& Weld realizaram um experimento envolvendo o planejador quantitativo GPT [Bonet \& Geffner, 2005] e o planejador qualitativo MBP [Bertoli et al., 2001], no qual eles constatam que o tempo que o MBP gasta para resolver um problema de planejamento é, em geral, muito menor que o tempo que o GPT gasta para resolver o problema correspondente; porém, a solução encontrada pelo MBP é geralmente muito menos eficiente que aquela encontrada pelo GPT. Motivados por essa observação, eles propuseram o arcabouço de planejamento híbrido HYBPLAN [Mausam et al., 2007], que combina os planejadores GPT e MBP. Nesse arcabouço, inicialmente o GPT é chamado com um limite de tempo máximo. Após esse tempo, se o valor do estado inicial convergiu, a solução encontrada pelo GPT é devolvida como solução final pelo НувPLAN; caso contrário, vários estados foram rotulados como resolvidos pelo GPT (como faz o algoritmo LRTDP [Bonet \& Geffner, 2003b]) e uma política ótima parcial $\pi_{\mathrm{GPT}}$ é obtida. Então, uma chamada é feita ao MBP e a política obtida, digamos $\pi_{\mathrm{MBP}}$, é combinada com a política parcial $\pi_{\mathrm{GPT}}$. Usando as informações nessa política combinada, HYBPLAN melhora a heurística empregada para guiar as simulações efetuadas pelo GPT e chama esse planejador novamente. Esse processo é repetido até que o GPT consiga obter uma política ótima completa, dentro do limite de tempo máximo estipulado.

Conforme observamos, a idéia básica do arcabouço de planejamento híbrido proposto em [Mausam et al., 2007] é a mesma do algoritmo de planejamento probabilístico forte PPF, que apresentamos na Subseção 6.3; ou seja, combinar o princípio de otimalidade de Bellman com técnicas de verificação de modelos. Porém, em PPF, não fazemos uso de heurísticas e operações básicas de computação de pré-imagem e valores ótimos são executadas de forma intercalada. De qualquer forma, acreditamos que os resultados apresentados em [Mausam et al., 2007] servem para reforçar a conjectura sobre a eficiência do nosso algoritmo. 
Iteração de valor topológica. Iteração de valor [Puterman, 1994] é um algoritmo bastante utilizado para solução de MDPs. Apesar disso, devido ao grande número de atualizações de valores de estados que ele efetua (muitas das quais redundantes), esse algoritmo pode ser extremamente ineficiente. Essa ineficiência resulta essencialmente do fato da iteração de valor não explorar a estrutura gráfica do MDP sendo resolvido que, conforme [Littman et al., 1995], é decisiva para a complexidade da sua solução.

Motivados por essa observação, Dai \& Goldsmith propuseram um algoritmo denominado TVi (Topological Value Iteration) [Dai \& Goldsmith, 2007]. A idéia básica desse algoritmo é levar em conta o fato de que existe uma relação causal entre estados de um MDP. Assim, se um estado $s^{\prime}$ pode ser alcançado após a execução de uma ação $a$ num estado $s$, então $V(s)$ depende de $V\left(s^{\prime}\right)$ e, portanto, atualizar $V\left(s^{\prime}\right)$ antes de $V(s)$ pode diminuir bastante o número de atualizações de valores de estados. Porém, um fato que impede a aplicação direta dessa idéia é que MDPs, em geral, possuem ciclos e, portanto, seus estados não podem ser ordenados topologicamente. Para contornar esse problema, dado um MDP cíclico $\mathcal{M}$, o algoritmo TVI deriva um novo MDP acíclico $\mathcal{M}^{\prime}$ correspondente, tal que todo componente fortemente conexo em $\mathcal{M}$ dá origem a um único meta-estado em $\mathcal{M}^{\prime}$. Então, para resolver o MDP derivado, TVI utiliza uma ordem topológica reversa para atualização de valores, chamando o algoritmo de iteração de valor (localmente) sempre que um meta-estado é encontrado. Conforme mostram os experimentos realizados em [Dai \& Goldsmith, 2007], além de ser mais eficiente que iteração de valor, o algoritmo TVI também tem desempenho superior ao de outros algoritmos que são considerados o estado-da-arte (LAO ${ }^{\star}$ [Hansen \& Zilberstein, 2001], LRTDP [Bonet \& Geffner, 2003b] e HDP [Bonet \& Geffner, 2003a]).

Conforme observamos, o algoritmo de planejamento probabilístico forte PPF, apresentado no Capítulo 6, além de gerar políticas parciais sobre $\mathcal{S}$, também atualiza os valores dos estados em ordem topológica reversa. Assim, embora não tenhamos realizado experimentos comparativos entre PPF e outros algoritmos para solução de MDPs, acreditamos que o algoritmo PPF tenha um desempenho comparável àquele do algoritmo TVI e, conseqüentemente, também seja bastante eficiente. Além disso, independentemente da questão de eficiência, nenhum dos algoritmos comparados em [Dai \& Goldsmith, 2007] é capaz de garantir soluções fortes (como faz o algoritmo PPF proposto nessa tese). 


\subsection{Contribuições desse trabalho}

As principais contribuições desse trabalho são:

- definição de uma classe de metas mais expressivas que aquelas do planejamento clássico (denominadas metas de alcançabilidade estendidas) que, além de especificar estados metas, também permitem a especificação de restrições sobre os caminhos de execução da política (conforme ressaltamos na Subseção 1.2.1, essa classe de metas ainda não havia sido devidamente tratada pela comunidade de planejamento);

- definição de uma lógica temporal cuja semântica permite o tratamento da classe de metas de alcançabilidade estendidas (conforme mostramos na Seção 5.2, a lógica CTL, que pode ser usada para especificar metas de alcançabilidade simples, não é adequada para o tratamento de metas de alcançabilidade estendidas);

- formulação de um arcabouço formal para resolver problemas de planejamento qualitativo em domínios não-determinísticos e completamente observáveis, envolvendo metas de alcançabilidade estendidas (até onde sabemos, esse é o primeiro trabalho que propõe a implementação de métodos para tratamento de metas de alcançabilidade estendidas, capaz de garantir a qualidade das soluções durante a sua síntese);

- formulação de um algoritmo para planejamento probabilístico forte para metas de alcançabilidade estendidas, que integra idéias de planejamento qualitativo e quantitativo.

\subsection{Trabalhos futuros}

Algumas extensões importantes desse trabalho são:

- Comparar as lógicas $\alpha$-CTL e CTL com relação à expressividade e à complexidade. Acreditamos que, embora $\alpha$-CTL seja mais sucinta que CTL, ambas as lógicas tenham a mesma complexidade computacional.

- Verificar a possibilidade de usar $\alpha$-CTL [Pereira \& Barros, 2007c] para tratar problemas de planejamento envolvendo metas do tipo "try its best", propostas em [Baral \& Zhao, 2006]. De fato, como mostramos em [Pereira \& Barros, 2007b], algumas dessas metas podem ser especificadas em $\alpha$-CTL; no entanto, ainda não temos algoritmos capazes de sintetizar soluções a partir dessas especificações. 
- Realizar uma análise comparativa de desempenho dos algoritmos PPF [Pereira et al., 2007] e VI [Puterman, 1994]. Com essa análise, esperamos mostrar que, para problemas de planejamento em domínios selecionados, o algoritmo PPF é mais eficiente que VI.

- Investigar o uso de $\alpha$-CTL para a implementação de funções de recompensas em MDPs. Acreditamos que, usando o algoritmo de verificação de modelos para $\alpha$-CTL, poderíamos atribuir recompensas aos estados de um MDP, antes que um método convencional para solução desse MDP fosse empregado. Como consequiência disso, teríamos especificações de meta de mais alto nível e ainda poderíamos reduzir o tamanho do MDP considerado durante a síntese da política. Nessa linha de pesquisa, também seria necessário realizar testes empíricos que comprovassem a capacidade de fórmulas $\alpha$-CTL expressarem metas estendidas num MDP. 


\section{Apêndice A}

\section{Provas dos teoremas}

Nesse apêndice, apresentamos as provas para os teoremas dos Capítulos 5 e 6 .

\section{A.1 Propriedades formais do algoritmo VACTL}

Teorema 5.1. Dados um modelo $\mathcal{D}$ com assinatura $(\mathbb{P}, \mathbb{A})$ e uma fórmula $\alpha$-CTL $\varphi$, a função INTENSÃO $[\mathcal{D}](\varphi)$ devolve o conjunto de estados $\llbracket \varphi \rrbracket_{\mathcal{D}}$.

Prova. A demonstração é feita por indução sobre a estrutura da fórmula $\varphi$. Para simplificar, vamos suprimir da nossa notação a indicação do domínio $\mathcal{D}$.

1. Base. A base da indução estabelece o resultado para fórmulas atômicas. Se $\varphi \in \mathbb{P}$, então a função $\operatorname{Intens} \tilde{A} O(\varphi)$ devolve o conjunto $\{s \in \mathcal{S}: \varphi \in \mathcal{L}(s)\}$ que, de acordo com a Definição 5.6, é justamente o conjunto de estados $\llbracket \varphi \rrbracket$.

2. Passo. Suponha que a hipótese de indução vale para subfórmulas de $\varphi$.

(a) IntENSÃo $\left(\neg \varphi_{1}\right)$ devolve $\mathcal{S} \backslash \operatorname{InTENSÃo}\left(\varphi_{1}\right)$. Mas, pela hipótese de indução, temos que $\mathcal{S} \backslash \operatorname{InTEns} \tilde{O} O\left(\varphi_{1}\right)=\mathcal{S} \backslash \llbracket \varphi_{1} \rrbracket$ e, de acordo com a Definição $5.6, \mathcal{S} \backslash \llbracket \varphi_{1} \rrbracket=\llbracket \neg \varphi_{1} \rrbracket$.

(b) $\operatorname{InTENS} \tilde{A} O\left(\varphi_{1} \wedge \varphi_{2}\right)$ devolve $\operatorname{InTENS} \tilde{A} O\left(\varphi_{1}\right) \cap \operatorname{IntEnSÃO}\left(\varphi_{2}\right)$. Mas, pela hipótese de indução, temos que $\operatorname{InTENSÃO}\left(\varphi_{1}\right) \cap \operatorname{InTENS} \tilde{\mathrm{A} O}\left(\varphi_{2}\right)=\llbracket \varphi_{1} \rrbracket \cap \llbracket \varphi_{2} \rrbracket$ e, de acordo com a Definição 5.6, $\llbracket \varphi_{1} \rrbracket \cap \llbracket \varphi_{2} \rrbracket=\llbracket \varphi_{1} \wedge \varphi_{2} \rrbracket$.

(c) $\operatorname{Intens} \tilde{A} O\left(\varphi_{1} \vee \varphi_{2}\right)$ devolve $\operatorname{Intens} \tilde{A} O\left(\varphi_{1}\right) \cup \operatorname{InTEnSÃo}\left(\varphi_{2}\right)$. Mas, pela hipótese de indução, temos que $\operatorname{InTENSÃO}\left(\varphi_{1}\right) \cup \operatorname{InTENSÃo}\left(\varphi_{2}\right)=\llbracket \varphi_{1} \rrbracket \cup \llbracket \varphi_{2} \rrbracket$ e, de acordo com a Definição 5.6, $\llbracket \varphi_{1} \rrbracket \cup \llbracket \varphi_{2} \rrbracket=\llbracket \varphi_{1} \vee \varphi_{2} \rrbracket$. 
(d) Intensão $\left(\exists \odot \varphi_{1}\right)$ devolve $\mathcal{T}_{\exists}^{-}\left(\operatorname{Intens} \tilde{a} O\left(\varphi_{1}\right)\right)$. Mas, pela hipótese de indução, temos que $\mathcal{T}_{\exists}^{-}\left(\operatorname{InTEns} \tilde{\mathrm{I} O}\left(\varphi_{1}\right)\right)=\mathcal{T}_{\exists}^{-}\left(\llbracket \varphi_{1} \rrbracket\right)$ e, de acordo com a Definição 5.6, $\mathcal{T}_{\exists}^{-}\left(\llbracket \varphi_{1} \rrbracket\right)=\llbracket \exists \odot \varphi_{1} \rrbracket$.

(e) INTENSÃo $\left(\forall \odot \varphi_{1}\right)$ devolve $\mathcal{T}_{\forall}^{-}\left(\operatorname{InTENSÃo}\left(\varphi_{1}\right)\right)$ que, analogamente ao caso anterior, corresponde a $\llbracket \forall \odot \varphi_{1} \rrbracket$.

(f) $\operatorname{IntEns} \tilde{A} O\left(\exists \boxminus \varphi_{1}\right)$ devolve Intens ÃoEG $\left(\varphi_{1}\right)$. Vamos mostrar que $\operatorname{IntEns} \tilde{\operatorname{IaEG}}\left(\varphi_{1}\right)$ $=\llbracket \exists \boxminus \varphi_{1} \rrbracket$. Pela hipótese de indução, a linha 1 do algoritmo InTEnsãoEG define $I=\llbracket \varphi_{1} \rrbracket$. Ademais, a cada iteração do laço na linha 3, a variável $I$ é atualizada pela atribuição $I \leftarrow I \cap \mathcal{T}_{\exists}^{-}(I)$, que corresponde ao funcional $\Gamma[I]=\llbracket \varphi_{1} \rrbracket \cap \mathcal{T}_{\exists}^{-}(I)$. Como $\Gamma[I]$ é monotônico sobre o reticulado $2^{\mathcal{S}}$ e o conjunto $\mathcal{S}$ é finito, do Teorema 2.1, segue que $\Gamma[I]$ tem um ponto-fixo máximo $\nu I .\left(\llbracket \varphi_{1} \rrbracket \cap \mathcal{T}_{\exists}^{-}(I)\right)$, que é o conjunto devolvido pela função IntensãoEG $\left(\varphi_{1}\right)$. Mas, de acordo com a Definição 5.6, $\nu I .\left(\llbracket \varphi_{1} \rrbracket \cap \mathcal{T}_{\exists}^{-}(I)\right)=\llbracket \exists \boxminus \varphi_{1} \rrbracket$.

(g) Intensão $\left(\forall \bullet \varphi_{1}\right)$ devolve $\operatorname{InTEnSÃoAG}\left(\varphi_{1}\right)$ que, analogamente ao caso anterior corresponde a $\llbracket \forall \bullet \varphi_{1} \rrbracket$.

(h) $\operatorname{Intensão}\left(\exists\left(\varphi_{1} \bullet \varphi_{2}\right)\right)$ devolve o conjunto $\operatorname{IntEns} \tilde{\operatorname{OaEU}}\left(\varphi_{1}, \varphi_{2}\right)$. Vamos mostrar que IntensãoEU $\left(\varphi_{1}, \varphi_{2}\right)=\llbracket \exists\left(\varphi_{1} \bullet \varphi_{2}\right) \rrbracket$. Pela hipótese de indução, as linhas 1 e 2 do algoritmo InTENSÃoEU definem $I_{1}=\llbracket \varphi_{1} \rrbracket$ e $I_{2}=\llbracket \varphi_{2} \rrbracket$, respectivamente. Ademais, a cada iteração do laço na linha 4, o conjunto $I_{2}$ é atualizado pela atribuição $I_{2} \leftarrow I_{2} \cup\left(I_{1} \cap \mathcal{T}_{\exists}^{-}\left(I_{2}\right)\right)$, que corresponde ao funcional $\Gamma\left[I_{2}\right]=\llbracket \varphi_{2} \rrbracket \cup\left(\llbracket \varphi_{1} \rrbracket \cap\right.$ $\left.\mathcal{T}_{\exists}^{-}\left(I_{2}\right)\right)$ ). Como $\Gamma\left[I_{2}\right]$ é monotônico sobre o reticulado $2^{\mathcal{S}}$ e o conjunto $\mathcal{S}$ é finito, do Teorema 2.1 , segue que $\Gamma\left[I_{2}\right]$ tem um ponto-fixo mínimo $\mu I_{2} \cdot\left(\llbracket \varphi_{2} \rrbracket \cup\left(\llbracket \varphi_{1} \rrbracket \cap\right.\right.$ $\left.\mathcal{T}_{\exists}^{-}\left(I_{2}\right)\right)$ ), que é o conjunto devolvido pela função InTEnsãoEU. Mas, de acordo com a Definição 5.6, $\mu I_{2} \cdot\left(\llbracket \varphi_{2} \rrbracket \cup\left(\llbracket \varphi_{1} \rrbracket \cap \mathcal{T}_{\exists}^{-}\left(I_{2}\right)\right)\right)=\llbracket \exists\left(\varphi_{1} \bullet \varphi_{2}\right) \rrbracket$.

(i) INTENSÃo $\left(\forall\left(\varphi_{1} \bullet \varphi_{2}\right)\right)$ devolve o conjunto InTENSÃOAU $\left(\varphi_{1}, \varphi_{2}\right)$ que, analogamente ao caso anterior, corresponde a $\llbracket \exists\left(\varphi_{1} \bullet \varphi_{2}\right) \rrbracket$.

(j) $\operatorname{Intens} \tilde{A} O\left(\exists \odot \varphi_{2}\right)$ devolve Intens ÃoEU $\left(T, \varphi_{2}\right)$ que, como foi demonstrado no caso (h), corresponde a $\llbracket \exists\left(\top \sqcup \varphi_{2}\right) \rrbracket$, que também pode ser escrito como $\llbracket \exists \diamond \varphi_{2} \rrbracket$.

(k) InTENSÃo $\left(\forall \odot \varphi_{2}\right)$ devolve InTENSÃOAU $\left(T, \varphi_{2}\right)$ que, analogamente ao caso anterior, corresponde a $\llbracket \forall \odot \varphi_{2} \rrbracket$. 
Corolário 5.1. Dados um modelo $\mathcal{D}$ com assinatura $(\mathbb{P}, \mathbb{A})$ e uma fórmula $\alpha$-CTL $\varphi$, o algoritmo $\operatorname{VActL}(\mathcal{D}, \varphi)$ sucede se e somente se todo estado em $\mathcal{D}$ satisfaz $\varphi$.

Prova. A atribuição na primeira linha do algoritmo $\operatorname{Vactl}$ define $C=\mathcal{S} \backslash \operatorname{Intens}$ Ão $(\varphi)$ e, pelo Teorema 5.1, $C=\mathcal{S} \backslash \llbracket \varphi \rrbracket_{\mathcal{D}}$. Então, se todo estado em $\mathcal{D}$ satisfaz $\varphi, C=\varnothing \mathrm{e}$, portanto, o algoritmo VACTL sucede. Por outro lado, se o algoritmo fracassa (devolvendo o contra-exemplo $C \neq \varnothing$ como resposta), então não é verdade que todo estado em $\mathcal{D}$ satisfaz $\varphi$.

\section{A.2 Propriedades formais do algoritmo PACTL}

Definição A.1. A distância fraca entre um estado s e um conjunto de estados $C$, denotada por $\delta(s, C)$, é o menor $i \in \mathbb{N}$ tal que existe um caminho de comprimento $i$ de s a algum $s^{\prime} \in C$. Se $s \in C$, definimos $\delta(s, C)=0$. Se não existe caminho de $s$ a $s^{\prime} \in C$, definimos $\delta(s, C)=\infty$.

Definição A.2. A distância forte entre um estado s e um conjunto de estados $C$, denotada por $\Delta(s, C)$, é definida como 0 , se $s \in C$; ou, então, é o menor $i \in \mathbb{N}$ tal que, para alguma ação $a \in \mathbb{A}(s)$ e para todo $s^{\prime} \in \mathcal{T}(s, a)$, a distância forte entre $s^{\prime}$ e $C$ é menor ou igual a $i-1$. Caso não exista $i \in \mathbb{N}$ que satisfaça essa condição, definimos $\Delta(s, C)=\infty$.

Lema A.1. Dados um domínio de planejamento $\mathcal{D}$ com assinatura $(\mathbb{P}, \mathbb{A})$ e uma fórmula proposicional $\varphi$ sobre $\mathbb{P}$, a função MODELo $[\mathcal{D}](e, \varphi)$ devolve o conjunto de estados $\llbracket \varphi \rrbracket_{\mathcal{D}}$.

Prova. A demonstração é feita por indução sobre a estrutura da fórmula $\varphi$. Para simplificar a notação, deixaremos implícitos o domínio $\mathcal{D}$ e o escopo $e$.

1. Base. Se $\phi \in \mathbb{P}$, então a função $\operatorname{Modelo}(\varphi)$ devolve o conjunto $\{s \in \mathcal{S}: \varphi \in \mathcal{L}(s)\}$ que, de acordo com Definição 5.6, é o conjunto de estados $\llbracket \varphi \rrbracket$.

2. Passo. Suponha que a hipótese de indução vale para subfórmulas de $\varphi$.

(a) $\operatorname{Modelo}\left(\neg \varphi_{1}\right)$ devolve o conjunto $\mathcal{S} \backslash \operatorname{Modelo}\left(\varphi_{1}\right)$. Mas, pela hipótese de indução, $\mathcal{S} \backslash \operatorname{Modelo}\left(\varphi_{1}\right)=\mathcal{S} \backslash \llbracket \varphi_{1} \rrbracket$ e, de acordo com a Definição 5.6, $\mathcal{S} \backslash \llbracket \varphi_{1} \rrbracket=\llbracket \neg \varphi_{1} \rrbracket$. 
(b) $\operatorname{Modelo}\left(\varphi_{1} \wedge \varphi_{2}\right)$ devolve o conjunto $\operatorname{Modelo}\left(\varphi_{1}\right) \cap \operatorname{Modelo}\left(\varphi_{2}\right)$. Mas, pela hipótese de indução, $\operatorname{Modelo}\left(\varphi_{1}\right) \cap \operatorname{Modelo}\left(\varphi_{2}\right)=\llbracket \varphi_{1} \rrbracket \cap \llbracket \varphi_{2} \rrbracket$ e, de acordo com a Definição 5.6, $\llbracket \varphi_{1} \rrbracket \cap \llbracket \varphi_{2} \rrbracket=\llbracket \varphi_{1} \wedge \varphi_{2} \rrbracket$.

(c) $\operatorname{Modelo}\left(\varphi_{1} \vee \varphi_{2}\right)$ devolve o conjunto $\operatorname{Modelo}\left(\varphi_{1}\right) \cup \operatorname{Modelo}\left(\varphi_{2}\right)$. Mas, pela hipótese de indução, $\operatorname{Modelo}\left(\varphi_{1}\right) \cup \operatorname{Modelo}\left(\varphi_{2}\right)=\llbracket \varphi_{1} \rrbracket \cup \llbracket \varphi_{2} \rrbracket$ e, de acordo com a Definição 5.6, $\llbracket \varphi_{1} \rrbracket \cup \llbracket \varphi_{2} \rrbracket=\llbracket \varphi_{1} \vee \varphi_{2} \rrbracket$.

Lema A.2. Ao final da i-ésima iteração da função ModeloEU $[\mathcal{D}]\left(\min , \varphi_{1}, \varphi_{2}\right)$, o modelo $M_{2}$ cobre todo estado $s \in \mathcal{S}$ tal que $\delta\left(s, \llbracket \varphi_{2} \rrbracket\right)=0$ ou $s \in \llbracket \varphi_{1} \rrbracket$ e $\delta\left(s, \llbracket \varphi_{2} \rrbracket\right) \leq i$.

Prova. Pelo Lema A.1, no início da primeira iteração da função ModeloEU, $M_{2}=\llbracket \varphi_{2} \rrbracket$ e, portanto, $M_{2}$ cobre todo estado $s \in \mathcal{S}$ tal que $\delta\left(s, \llbracket \varphi_{2} \rrbracket\right)=0$. Computando a pré-imagem fraca da cobertura de $M_{2}$, obtemos $I_{2}$, que cobre todo $s \in \mathcal{S}$ que tem pelo menos um sucessor em $\llbracket \varphi_{2} \rrbracket$. Em seguida, chamando a função $\operatorname{PodAU}\left(\min , I_{2}, \ldots\right)$, obtemos $P_{2}$, resultante da eliminação de $I_{2}$ de todos os estados que não estão em $\llbracket \varphi_{1} \rrbracket$ ou que estão em $\llbracket \varphi_{2} \rrbracket$; ou seja, $P_{2}$ cobre todo estado $s \in \mathcal{S}$ tal que $s \in \llbracket \varphi_{1} \rrbracket$ e $\delta\left(s, \llbracket \varphi_{2} \rrbracket\right)=1$. Portanto, após a execução da atribuição $M_{2} \leftarrow M_{2} \cup P_{2}$, no final da primeira iteração, o modelo $M_{2}$ cobre todo estado $s \in \mathcal{S}$ tal que $\delta\left(s, \llbracket \varphi_{2} \rrbracket\right)=0$ ou $s \in \llbracket \varphi_{1} \rrbracket$ e $\delta\left(s, \llbracket \varphi_{2} \rrbracket\right) \leq 1$. Suponha que no início da $i$-ésima iteração $(i \geq 2), M_{2}$ cobre todo estado $s \in \mathcal{S}$ tal que $\delta\left(s, \llbracket \varphi_{2} \rrbracket\right)=0$ ou $s \in \llbracket \varphi_{1} \rrbracket$ e $\delta\left(s, \llbracket \varphi_{2} \rrbracket\right) \leq(i-1)$. Então, quando a préimagem fraca da cobertura de $M_{2}$ é computada na $i$-ésima iteração, obtemos o conjunto $I_{2}$, que cobre todo $s \in \mathcal{S}$ que tem pelo menos um sucessor em $M_{2}$. Em seguida, chamando a função $\operatorname{PodAU}\left(\min , I_{2}, \ldots\right)$, eliminamos de $I_{2}$ todos os estados que não estão em $\llbracket \varphi_{1} \rrbracket$ ou que estão em $M_{2}$, e obtemos o conjunto $P_{2}$. Assim, claramente, se $s \in P_{2}$, no mínimo uma transição a partir de $s$ é necessária para alcançar um estado $s^{\prime} \in M_{2}$. Pela hipótese de indução, $M_{2}$ cobre todo estado $s^{\prime} \in \mathcal{S}$ tal que $\delta\left(s^{\prime}, \llbracket \varphi_{2} \rrbracket\right)=0$ ou $s^{\prime} \in \llbracket \varphi_{1} \rrbracket$ e $\delta\left(s^{\prime}, \llbracket \varphi_{2} \rrbracket\right) \leq(i-1)$. Então, como $P_{2} \cap M_{2}=\varnothing$, concluímos que $P_{2}$ cobre todo estado $s \in \mathcal{S}$ tal que $\delta\left(s, \llbracket \varphi_{2} \rrbracket\right)=i$. Assim, após a execução da atribuição $M_{2} \leftarrow M_{2} \cup P_{2}$, no final da $i$-ésima iteração, temos que $M_{2}$ cobre todo estado $s \in \mathcal{S}$ tal que $\delta\left(s, \llbracket \varphi_{2} \rrbracket\right)=0$ ou $s \in \llbracket \varphi_{1} \rrbracket$ e $\delta\left(s, \llbracket \varphi_{2} \rrbracket\right) \leq i$.

Lema A.3. Ao final da i-ésima iteração da função ModeloAU $[\mathcal{D}]\left(\min , \varphi_{1}, \varphi_{2}\right)$, o modelo $M_{2}$ cobre todo estado $s \in \mathcal{S}$ tal que $\Delta\left(s, \llbracket \varphi_{2} \rrbracket\right)=0$ ou $s \in \llbracket \varphi_{1} \rrbracket$ e $\Delta\left(s, \llbracket \varphi_{2} \rrbracket\right) \leq i$.

Prova. Pelo Lema A.1, no início da primeira iteração da função ModeloAU, $M_{2}=\llbracket \varphi_{2} \rrbracket \mathrm{e}$, portanto, $M_{2}$ cobre todo estado $s \in \mathcal{S}$ tal que $\Delta\left(s, \llbracket \varphi_{2} \rrbracket\right)=0$. Computando a pré-imagem forte 
da cobertura de $M_{2}$, obtemos $I_{2}$, que cobre todo estado $s \in \mathcal{S}$ cujos $a$-sucessores (para alguma ação $a \in \mathbb{A}(s))$ estão todos em $\llbracket \varphi_{2} \rrbracket$. Em seguida, chamando a função $\operatorname{PodAU}\left(\min , I_{2}, \ldots\right)$, obtemos $P_{2}$, resultante da eliminação de $I_{2}$ de todos os estados que não estão em $\llbracket \varphi_{1} \rrbracket$ ou que estão $\llbracket \varphi_{2} \rrbracket$; ou seja, $P_{2}$ cobre todo estado $s \in \mathcal{S}$ tal que $s \in \llbracket \varphi_{1} \rrbracket$ e $\Delta\left(s, \llbracket \varphi_{2} \rrbracket\right)=1$. Assim, após a execução da atribuição $M_{2} \leftarrow M_{2} \cup P_{2}$, no final da primeira iteração, $M_{2}$ cobre todo estado $s \in \mathcal{S}$ tal que $\Delta\left(s, \llbracket \varphi_{2} \rrbracket\right)=0$ ou $s \in \llbracket \varphi_{1} \rrbracket$ e $\Delta\left(s, \llbracket \varphi_{2} \rrbracket\right) \leq 1$. Suponha que no início da $i$-ésima iteração $(i \geq 2), M_{2}$ cobre todo estado $s \in \mathcal{S}$ tal que $\Delta\left(s, \llbracket \varphi_{2} \rrbracket\right)=0$ ou $s \in \llbracket \varphi_{1} \rrbracket$ e $\Delta\left(s, \llbracket \varphi_{2} \rrbracket\right) \leq(i-1)$. Então, quando a pré-imagem forte de $M_{2}$ é computada na $i$-ésima iteração, obtemos o conjunto $I_{2}$, que cobre todo estado $s \in \mathcal{S}$ cujos $a$-sucessores (para alguma ação $a \in \mathbb{A}(s)$ ) estão todos em $M_{2}$. Em seguida, chamando a função $\operatorname{PodaU}\left(\min , I_{2}, \ldots\right)$, eliminamos de $I_{2}$ todos os estados que não estão em $\llbracket \varphi_{1} \rrbracket$ ou que estão em $M_{2}$, e obtemos $P_{2}$. Assim, claramente, se $s \in P_{2}$, no mínimo uma transição a partir de $s$ é necessária para alcançar um estado $s^{\prime} \in M_{2}$. Pela hipótese de indução, $M_{2}$ cobre todo estado $s \in \mathcal{S}$ tal que $\Delta\left(s^{\prime}, \llbracket \varphi_{2} \rrbracket\right)=0$ ou $s^{\prime} \in \llbracket \varphi_{1} \rrbracket$ e $\Delta\left(s^{\prime}, \llbracket \varphi_{2} \rrbracket\right) \leq(i-1)$. Então, como $P_{2} \cap M_{2}=\varnothing$, concluímos que $P_{2}$ cobre todo $s \in \mathcal{S}$ tal que $\Delta\left(s, \llbracket \varphi_{2} \rrbracket\right)=i$. Assim, após a execução da atribuição $M_{2} \leftarrow M_{2} \cup P_{2}$, no final da $i$-ésima iteração, temos que $M_{2}$ cobre todo estado $s \in \mathcal{S}$ tal que $\Delta\left(s, \llbracket \varphi_{2} \rrbracket\right)=0$ ou $s \in \llbracket \varphi_{1} \rrbracket$ e $\Delta\left(s, \llbracket \varphi_{2} \rrbracket\right) \leq i$.

Proposição A.1. Dados um domínio de planejamento $\mathcal{D}$ com assinatura $(\mathbb{P}, \mathbb{A})$ e uma fórmula $\alpha$-CTL $\varphi=\exists\left(\varphi_{1} \sqcup \varphi_{2}\right)$, sendo $\varphi_{1}$ e $\varphi_{2}$ fórmulas proposicionais sobre $\mathbb{P}$, a função $\operatorname{ModeLo}(e, \varphi)$ devolve um submodelo $M \subseteq \mathcal{D}$, tal que CobertuRa $(M)=\llbracket \exists\left(\varphi_{1} \sqcup \varphi_{2}\right) \rrbracket_{\mathcal{D}}$.

Prova. Claramente, para $\varphi=\exists\left(\varphi_{1} \sqcup \varphi_{2}\right)$, a chamada à função Modelo devolve um submodelo $M \subseteq \mathcal{D}$, sendo $M=\operatorname{ModeloEU}\left(e, \varphi_{1}, \varphi_{2}\right)$. Pela Lema A.2, ao final da $i$-ésima iteração da função ModeloEU, obtemos um modelo $M$ que cobre todo estado $s \in \mathcal{S}$ tal que $\delta\left(s, \llbracket \varphi_{2} \rrbracket\right)=0$ ou $s \in \llbracket \varphi_{1} \rrbracket$ e $\delta\left(s, \llbracket \varphi_{2} \rrbracket\right) \leq i$. Como o conjunto $\mathcal{S}$ é finito, garantimos que a função ModELoEU termina em no máximo $|\mathcal{S}|$ iterações. Então, após a última iteração da função ModELoEU, o modelo $M$, devolvido como resposta pela função, cobre todo estado $s \in \mathcal{S}$ tal que $\delta\left(s, \llbracket \varphi_{2} \rrbracket\right)=0$ ou $s \in \llbracket \varphi_{1} \rrbracket$ e $\delta\left(s, \llbracket \varphi_{2} \rrbracket\right) \leq|\mathcal{S}|$. Como nenhum caminho acíclico em $\mathcal{D}$ tem comprimento maior que $|\mathcal{S}|$, concluímos que $M$ cobre todo estado $s \in \mathcal{S}$, a partir do qual existe um caminho até $s^{\prime} \in \llbracket \varphi_{2} \rrbracket$, passando apenas por estados em $\llbracket \varphi_{1} \rrbracket$. Logo, Cobertura $(M)=\llbracket \exists\left(\varphi_{1} \sqcup \varphi_{2}\right) \rrbracket_{\mathcal{D}}$.

Proposição A.2. Dados um domínio de planejamento $\mathcal{D}$ com assinatura $(\mathbb{P}, \mathbb{A})$ e uma fórmula $\alpha$-CTL $\varphi=\forall\left(\varphi_{1} \sqcup \varphi_{2}\right)$, sendo $\varphi_{1}$ e $\varphi_{2}$ fórmulas proposicionais sobre $\mathbb{P}$, a função $\operatorname{Modelo}(e, \varphi)$ devolve um submodelo $M \subseteq \mathcal{D}$, tal que COBERTURA $(M)=\llbracket \forall\left(\varphi_{1} \dot{\sim} \varphi_{2}\right) \rrbracket_{\mathcal{D}}$. 
Prova. Claramente, para $\varphi=\exists\left(\varphi_{1} \sqcup \varphi_{2}\right)$, a chamada à função ModELo devolve um submodelo $M \subseteq \mathcal{D}$, sendo $M=\operatorname{ModeloAU}\left(e, \varphi_{1}, \varphi_{2}\right)$. Pelo Lema A.3, ao final da $i$-ésima iteração da função ModeloAU, obtemos um modelo $M$ que cobre todo estado $s \in \mathcal{S}$ tal que $\Delta\left(s, \llbracket \varphi_{2} \rrbracket\right)=0$ ou $s \in \llbracket \varphi_{1} \rrbracket$ e $\Delta\left(s, \llbracket \varphi_{2} \rrbracket\right) \leq i$. Como o conjunto $\mathcal{S}$ é finito, garantimos que a função ModELOAU termina em no máximo $|\mathcal{S}|$ iterações. Então, após a última iteração da função ModELOAU, o modelo $M$, devolvido como resposta da função, cobre todo estado $s \in \mathcal{S}$ tal que $\Delta\left(s, \llbracket \varphi_{2} \rrbracket\right)=0$ ou $s \in \llbracket \varphi_{1} \rrbracket$ e $\Delta\left(s, \llbracket \varphi_{2} \rrbracket\right) \leq|\mathcal{S}|$. Como nenhum caminho acíclico em $\mathcal{D}$ pode ter comprimento maior que $|\mathcal{S}|$, concluímos que $M$ cobre todo estado $s \in \mathcal{S}$, a partir do qual todo caminho $s=$ $s_{0}, a_{0}, \ldots, s_{k-1}, a_{k-1}, s_{k}=s^{\prime}$ em $\mathcal{D}$ (para $a_{i} \in \mathbb{A}\left(s_{i}\right)$ e $k \leq|\mathcal{S}|$ ) leva até $s^{\prime} \in \llbracket \varphi_{2} \rrbracket$, passando apenas por estados em $\llbracket \varphi_{1} \rrbracket$. Logo, CobertuRA $(M)=\llbracket \forall\left(\varphi_{1} \sqcup \varphi_{2}\right) \rrbracket_{\mathcal{D}}$.

Proposição A.3. Dados um domínio de planejamento $\mathcal{D}$ com assinatura $(\mathbb{P}, \mathbb{A})$ e uma fórmula $\alpha$-CTL $\varphi=\forall \boxminus \exists\left(\varphi_{1} \sqcup \varphi_{2}\right)$, sendo $\varphi_{1}$ e $\varphi_{2}$ fórmulas proposicionais sobre $\mathbb{P}$, a função $\operatorname{ModELO}(e, \varphi)$ devolve um submodelo $M \subseteq \mathcal{D}$, tal que CoBertura $(M)=\llbracket \forall \boxminus \exists\left(\varphi_{1} \sqcup \varphi_{2}\right) \rrbracket_{\mathcal{D}}$.

Prova. Para $\varphi=\forall \bullet \exists\left(\varphi_{1} \sqcup \varphi_{2}\right)$, a chamada à função ModElo devolve um submodelo $M \subseteq$ $\mathcal{D}$, sendo $M=\operatorname{ModeloAG}\left(\max , \exists\left(\varphi_{1} \bullet \varphi_{2}\right)\right)$. Vamos mostrar que $\llbracket \forall \bullet \exists\left(\varphi_{1} \sqcup \varphi_{2}\right) \rrbracket \subseteq$ Cobertura $(M)$ é o invariante do laço na função ModeloAG. A primeira linha dessa função define $M=\operatorname{Modelo}\left(e, \exists\left(\varphi_{1} \sqcup \varphi_{2}\right)\right)$ e, portanto, pela Proposição A.1, temos que Cobertura $(M)=$ $\llbracket \exists\left(\varphi_{1} \sqcup \varphi_{2}\right) \rrbracket$. Como $\forall \bullet \exists\left(\varphi_{1} \sqcup \varphi_{2}\right) \rrbracket \subseteq \llbracket \exists\left(\varphi_{1} \sqcup \varphi_{2}\right) \rrbracket$, claramente, o invariante vale no início da primeira iteração. Suponha, então, que o invariante vale no início da $i$-ésima iteração, para $i \geq 1$. Computando a pré-imagem forte da cobertura de $M$, obtemos $I$, que cobre todo estado $s \in \mathcal{S}$ cujos $a$-sucessores (para alguma ação $a \in \mathbb{A}(s)$ ) estão todos em $M$. Em seguida, chamando a função PodaG, o modelo $M$ é atualizado de modo que apenas estados cobertos, simultaneamente, por $I$ e por $M$ sejam mantidos em $M$. Note que, se $s_{i}$ está em $I$ e não está em $M$, então $s_{i}$ não satisfaz a fórmula $\exists\left(\varphi_{1} \sqcup \varphi_{2}\right)$ e, portanto, $s_{i} \notin \llbracket \forall \boxminus \exists\left(\varphi_{1} \sqcup \varphi_{2}\right) \rrbracket$. Por outro lado se $s_{m}$ está em $M$ e não está em $I$, então, para toda ação $a \in \mathbb{A}(s)$, algum $a$-sucessor de $s_{m}$ não satisfaz a fórmula $\exists\left(\varphi_{1} \bullet \varphi_{2}\right)$ e, portanto, $s_{m} \notin \llbracket \forall \boxminus \exists\left(\varphi_{1} \bullet \varphi_{2}\right) \rrbracket$. Isso significa que, a atualização do modelo $M$ não elimina nenhum estado pertencente ao conjunto $\llbracket \forall \exists\left(\varphi_{1} \sqcup \varphi_{2}\right) \rrbracket$ e, portanto, se o invariante vale no início da $i$-ésima iteração, claramente, ele continuará valendo no início da $(i+1)$-iteração. Como a cada iteração o modelo $M$ é reduzido, e o conjunto $\mathcal{S}$ é finito, após um número finito de iterações, um ponto-fixo máximo é obtido. Pela condição estabelecida pelo invariante, temos que $\llbracket \forall \boxminus \exists\left(\varphi_{1} \sqcup \varphi_{2}\right) \rrbracket \subseteq$ COBERTURA $(M)$ e, pela condição para obtenção de ponto-fixo máximo, temos que Cobertura $(M) \subseteq \llbracket \forall ๑ \exists\left(\varphi_{1} \sqcup \varphi_{2}\right) \rrbracket$. Logo, o submodelo $M \subseteq \mathcal{D}$, devolvido pela função ModeloAG, é tal que Cobertura $(M)=\llbracket \forall \boxminus \exists\left(\varphi_{1} \sqcup \varphi_{2}\right) \rrbracket_{\mathcal{D}}$. 
Teorema 5.2. Seja $\mathcal{P}=\left\langle\mathcal{D}, s_{0}, \exists\left(\varphi_{1} \sqcup \varphi_{2}\right)\right\rangle$ um problema de planejamento para meta de alcançabilidade estendida. Se a chamada $\operatorname{PaCtL}(\mathcal{P})$ devolve uma política $\pi$, então $\pi$ é uma solução fraca para o problema de planejamento $\mathcal{P}$.

Prova. As duas primeiras linhas do algoritmo Pactl definem $M=\operatorname{Modelo}\left(\min , \exists\left(\varphi_{1} \sqcup \varphi_{2}\right)\right)$ e $C=\operatorname{Cobertura}(M)$, respectivamente. Então, de acordo com a Proposição A.1, temos que $C=\llbracket \exists\left(\varphi_{1} \sqcup \varphi_{2}\right) \rrbracket_{\mathcal{D}}$. Ademais, devido ao comando condicional na terceira linha de PACTL, se a chamada $\operatorname{PaCtL}(\mathcal{P})$ devolve uma política $\pi$, concluímos que $s_{0} \in C$ e que, portanto, no modelo $M$ existe um caminho leva de $s_{0}$ até algum $s^{\prime} \in \llbracket \varphi_{2} \rrbracket$, passando apenas por estados em $\llbracket \varphi_{1} \rrbracket$. Ademais, pela definição da função PréImagemFracaMap, usada durante a construção do modelo $M$, sabemos que, para cada estado $s$ coberto por $M$, qualquer ação $a$ associada a $s$ em $M$ permite alcançar um estado $s^{\prime}$ tal que $s^{\prime} \in \llbracket \varphi_{2} \rrbracket$ (estado meta) ou, então, $s^{\prime} \in \llbracket \varphi_{1} \rrbracket$ e $s^{\prime}$ também é coberto por $M$ (ou seja, a partir de $s^{\prime}$ existe um caminho que leva a um estado meta). Logo, qualquer mapeamento $\pi$ de estados em ações extraído de $M$ é uma solução fraca para o problema de planejamento $\mathcal{P}$.

Teorema 5.3. Seja $\mathcal{P}=\left\langle\mathcal{D}, s_{0}, \forall\left(\varphi_{1} \sqcup \varphi_{2}\right)\right\rangle$ um problema de planejamento para meta de alcançabilidade estendida. Se a chamada $\operatorname{PACTL}(\mathcal{P})$ devolve uma política $\pi$, então $\pi$ é uma solução forte para o problema de planejamento $\mathcal{P}$.

Prova. As duas primeiras linhas do algoritmo Pactl definem $M=\operatorname{Modelo}\left(\min , \forall\left(\varphi_{1} \sqcup \varphi_{2}\right)\right)$ e $C=\operatorname{Cobertura}(M)$, respectivamente. Então, de acordo com a Proposição A.2, temos que $C=\llbracket \forall\left(\varphi_{1} \sqcup \varphi_{2}\right) \rrbracket_{\mathcal{D}}$. Ademais, devido ao comando condicional na terceira linha de PACTL, se a chamada $\operatorname{PaCtL}(\mathcal{P})$ devolve uma política $\pi$, concluímos que $s_{0} \in C$ e que, portanto, no modelo $M$ todo caminho leva de $s_{0}$ até algum $s^{\prime} \in \llbracket \varphi_{2} \rrbracket$, passando apenas por estados em $\llbracket \varphi_{1} \rrbracket$. Ademais, pela definição da função PrÉIMAGEMForteMAP, usada durante a construção do modelo $M$, sabemos que, para cada estado $s$ coberto por $M$, qualquer ação $a$ associada a $s$ em $M$ necessariamente alcança um estado $s^{\prime}$ tal que $s^{\prime} \in \llbracket \varphi_{2} \rrbracket$ (estado meta) ou, então, $s^{\prime} \in \llbracket \varphi_{1} \rrbracket$ e $s^{\prime}$ também é coberto por $M$ (ou seja, a partir de $s /$ todos os caminhos são acíclicos e levam a estados metas). Logo, qualquer mapeamento $\pi$ de estados em ações extraído de $M$ é uma solução forte para o problema de planejamento $\mathcal{P}$.

Teorema 5.4. Seja $\mathcal{P}=\left\langle\mathcal{D}, s_{0}, \forall \bullet \exists\left(\varphi_{1} \sqcup \varphi_{2}\right)\right\rangle$ um problema de planejamento para meta de alcançabilidade estendida. Se a chamada $\operatorname{PACtL}(\mathcal{P})$ devolve uma política $\pi$, então $\pi$ é uma solução forte-cíclica para o problema de planejamento $\mathcal{P}$. 
Prova. As duas primeiras linhas do algoritmo Pactl definem $M=\operatorname{Modelo}\left(\min , \forall \boxminus \exists\left(\varphi_{1} \sqcup\right.\right.$ $\left.\left.\varphi_{2}\right)\right)$ e $C=\operatorname{Cobertura}(M)$, respectivamente. Então, de acordo com a Proposição A.3, temos que $C=\llbracket \forall \boxminus \exists\left(\varphi_{1} \sqcup \varphi_{2}\right) \rrbracket_{\mathcal{D}}$. Ademais, devido ao comando condicional na terceira linha de PACtL, se a chamada $\operatorname{PACtL}(\mathcal{P})$ devolve uma política $\pi$, concluímos que $s_{0} \in C$ e que, portanto, no modelo $M$, ao longo de qualquer caminho que parte de $s_{0}$, existe sempre a possibilidade de alcançar $s^{\prime} \in \llbracket \varphi_{2} \rrbracket$, passando apenas por estados em $\llbracket \varphi_{1} \rrbracket$. Ademais, pela definição da função PrÉIMAgemFracaMap, usada durante a construção do modelo $M$, sabemos que, para cada estado $s$ coberto por $M$, qualquer ação $a$ associada a $s$ em $M$ permite alcançar um estado $s^{\prime}$ tal que $s^{\prime} \in \llbracket \varphi_{2} \rrbracket$ (estado meta) ou, então, $s^{\prime} \in \llbracket \varphi_{1} \rrbracket$ e $s^{\prime}$ também é coberto por $M$ (ou seja, ao longo de qualquer caminho que parte de $s^{\prime}$, existe sempre a possibilidade de alcançar um estado meta). Logo, qualquer mapeamento $\pi$ de estados em ações extraído de $M$ é uma solução forte-cíclica para o problema de planejamento $\mathcal{P}$.

Teorema 5.5. Seja $\mathcal{P}=\left\langle\mathcal{D}, s_{0}, \varphi\right\rangle$ um problema de planejamento para meta de alcançabilidade estendida. Então, a chamada $\operatorname{PACTL}(\mathcal{P})$ devolve fracasso se e somente se $\mathcal{P}$ não tem solução.

Prova. Se a fórmula $\alpha$-CTL $\varphi$ especifica uma meta de alcançabilidade estendida, de acordo com as Proposições A.1, A.2 e A.3, a segunda linha do algoritmo PACTL define $C=\llbracket \varphi \rrbracket_{\mathcal{D}}$. Suponha que a chamada $\operatorname{PACtL}(\mathcal{P})$ devolva fracasso. Então, devido ao condicional na terceira linha de PACтL, concluímos que $s_{0} \notin C=\llbracket \varphi \rrbracket_{\mathcal{D}}$, ou seja, o estado inicial $s_{0}$ não satisfaz a meta de planejamento especificada pela fórmula $\varphi$ e, portanto, o problema $\mathcal{P}$ não tem solução. Suponha agora que $\mathcal{P}$ tenha solução. Nesse caso, $s_{0}$ satisfaz a meta especificada pela fórmula $\varphi$ (ou seja, $\left.s_{0} \in \llbracket \varphi \rrbracket_{\mathcal{D}}=C\right)$ e, portanto, devido ao condicional na terceira linha do algoritmo, concluímos que a chamada $\operatorname{PACtL}(\mathcal{P})$ não devolve fracasso.

Teorema 5.6. O menor caminho gerado pela execução de uma política $\pi$, obtida por meio da chamada $\operatorname{PACTL}\left(\left\langle\mathcal{D}, s_{0}, \exists\left(\varphi_{1} \sqcup \varphi_{2}\right)\right\rangle\right)$, é mínimo no melhor caso.

Prova. Para sintetizar um submodelo $M \subseteq \mathcal{D}$ para a meta de planejamento especificada pela fórmula $\exists\left(\varphi_{1} \sqcup \varphi_{2}\right)$, algoritmo PACTL faz uma chamada à função ModeloEU. De acordo com o Lema A.2, após a $i$-ésima iteração dessa função, o modelo $M$ construído cobre todo estado $s \in \mathcal{S}$ tal que $\delta\left(s, \llbracket \varphi_{2} \rrbracket\right)=0$ ou $s \in \llbracket \varphi_{1} \rrbracket$ e $\delta\left(s, \llbracket \varphi_{2} \rrbracket\right) \leq i$. Suponha que o modelo $M$ sendo construído pela função ModeloEU cubra o estado $s_{0}$ apenas após a $i$-ésima iteração. Então, pela definição distância fraca, segue que $i$ é o menor inteiro positivo tal que existe um caminho 
de comprimento $i$ em $\mathcal{D}$, levando de $s_{0}$ até um estado meta $s^{\prime} \in \llbracket \varphi_{2} \rrbracket$. Logo, o menor caminho gerado pela execução de uma política $\pi$ extraída de $M$ é mínimo no melhor caso.

Teorema 5.7. O maior caminho gerado pela execução de uma política $\pi$, obtida por meio da chamada $\operatorname{PACTL}\left(\left\langle\mathcal{D}, s_{0}, \forall\left(\varphi_{1} \sqcup \varphi_{2}\right)\right\rangle\right)$, é mínimo no pior caso.

Prova. Para sintetizar um submodelo $M \subseteq \mathcal{D}$ para a meta de planejamento especificada pela fórmula $\forall\left(\varphi_{1} \sqcup \varphi_{2}\right)$, algoritmo PACTL faz uma chamada à função ModELOAU. De acordo com o Lema A.3, após a $i$-ésima iteração dessa função, o modelo $M$ construído cobre todo estado $s \in \mathcal{S}$ tal que $\Delta\left(s, \llbracket \varphi_{2} \rrbracket\right)=0$ ou $s \in \llbracket \varphi_{1} \rrbracket \mathrm{e} \Delta\left(s, \llbracket \varphi_{2} \rrbracket\right) \leq i$. Suponha que o modelo $M$ sendo construído pela função ModeloAU cubra o estado $s_{0}$ apenas após a $i$-ésima iteração. Então, pela definição distância forte, segue que $i$ é o menor inteiro positivo tal que todo caminho levando de $s_{0}$ até um estado meta $s^{\prime} \in \llbracket \varphi_{2} \rrbracket$ em $\mathcal{D}$ tem comprimento menor ou igual a $i$. Logo, o maior caminho gerado pela execução de uma política $\pi$ extraída de $M$ é mínimo no pior caso.

\section{A.3 Propriedades formais do algoritmo PPF}

Teorema 6.1. Se um problema de planejamento quantitativo $\mathcal{P}$ tem uma solução forte, então o algoritmo $\operatorname{PPF}(\mathcal{P})$ devolve uma política para $\mathcal{P}$ que é ótima no pior caso.

Prova. Seja $\pi_{i}$ a política construída ao final da $i$-ésima iteração do algoritmo PPF e seja $C_{i}$ a cobertura dessa política. Por definição, $\pi_{0}$ cobre apenas estados metas e, portanto, $C_{0}=\mathcal{G}$. Então, no início da primeira iteração, se $s_{0} \in C_{0}$, claramente, o algoritmo devolve uma política para $\mathcal{P}$ que é ótima no pior caso (i.e., o maior caminho de $s_{0}$ até um estado meta tem comprimento 0 ). Do contrário, a pré-imagem forte podada $P_{0}$ do conjunto $C_{0}$ é computada. Como, para $(s, a) \in P_{0}$, todos os $a$-sucessores de $s$ são estados metas; independentemente das ações escolhidas pela função EsCOLHA, a política

$\left.\pi_{1}:=\pi_{0} \cup \operatorname{Escolha}\left(\operatorname{Poda}\left(\operatorname{PrÉ} \operatorname{ImagemForteMap}\left(C_{0}\right), C_{0}\right)\right)\right)$,

construída ao final da primeira iteração, cobre todos os estados a partir dos quais, no pior caso, há um caminho de comprimento 1 para um estado meta. Pela hipótese de indução, para $j<i$, a política $\pi_{j}$ cobre todos os estados a partir dos quais, no pior caso, existe um caminho de comprimento $j$ para um estado meta. Assim, na $i$-ésima iteração, se o estado inicial $s_{0}$ é coberto 
por $\pi_{i-1}$, o algoritmo devolve uma política para $\mathcal{P}$, que é ótima no pior caso. Do contrário, a préimagem forte podada $P_{i-1}$ do conjunto $C_{i-1}$ é computada. Para cada $(s, a) \in P_{i-1}$, pelo menos um $a$-sucessor de $s$ precisa, no pior caso, de $i-1$ passos para alcançar um estado meta (pois, do contrário, o estado $s$ já teria sido coberto pela política $\pi_{i-1} \mathrm{e}$, portanto, teria sido podado). Logo, independentemente das ações escolhidas pela função EscoLHA, a política

$\left.\pi_{i}:=\pi_{i-1} \cup \operatorname{Escolha}\left(\operatorname{Poda}\left(\operatorname{PrÉ} \operatorname{ImagemForteMap}\left(C_{i-1}\right), C_{i-1}\right)\right)\right)$,

construída ao final da $i$-ésima iteração, cobre todos os estados a partir dos quais, no pior caso, existe um caminho de comprimento $i$ para um estado meta.

Teorema 6.2. A política devolvida pelo algoritmo $\operatorname{PPF}(\mathcal{P})$ é uma ótima no caso esperado, entre todas aquelas que são fortes e ótimas no pior caso.

Prova. A otimalidade no caso esperado da política devolvida pelo algoritmo PPF é uma conseqüência direta do fato da função EsCOLHA ser baseada em indução regressiva e usar o princípio da otimalidade como critério de escolha da melhor ação, para cada estado coberto pela política sendo construída. 


\section{Apêndice B}

\section{Verificação e síntese de modelos usando $\alpha$-CTL}

Nesse apêndice, mostramos como a lógica temporal de tempo ramificado $\alpha$-CTL pode ser utilizada para verificação e síntese de modelos. Para implementar os protótipos que apresentamos nas próximas seções, utilizamos a linguagem SWI-PROLOG, versão 5.6.38.

\section{B.1 Especificação de modelos}

Para especificar um modelo, utilizamos os seguintes predicados:

- label/2, que associa estados a conjuntos de proposições satisfeitas nesses estados;

- trans/3, que, para cada par de estado $s$ e ação $a$, estabelece os $a$-sucessores de $s$.

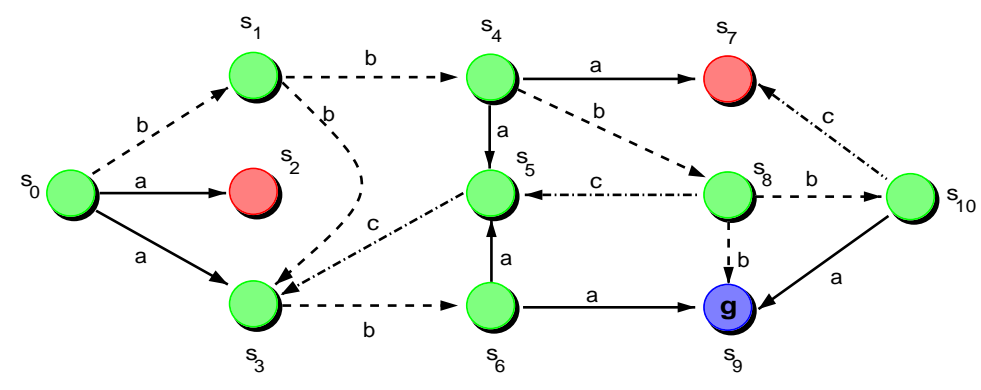

Figura B.1: Domínio de planejamento para meta de alcançabilidade simples $\mathcal{D}^{1}$. 
Exemplo B.1. O modelo apresentado na Figura B.1 pode ser especificado conforme a seguir:
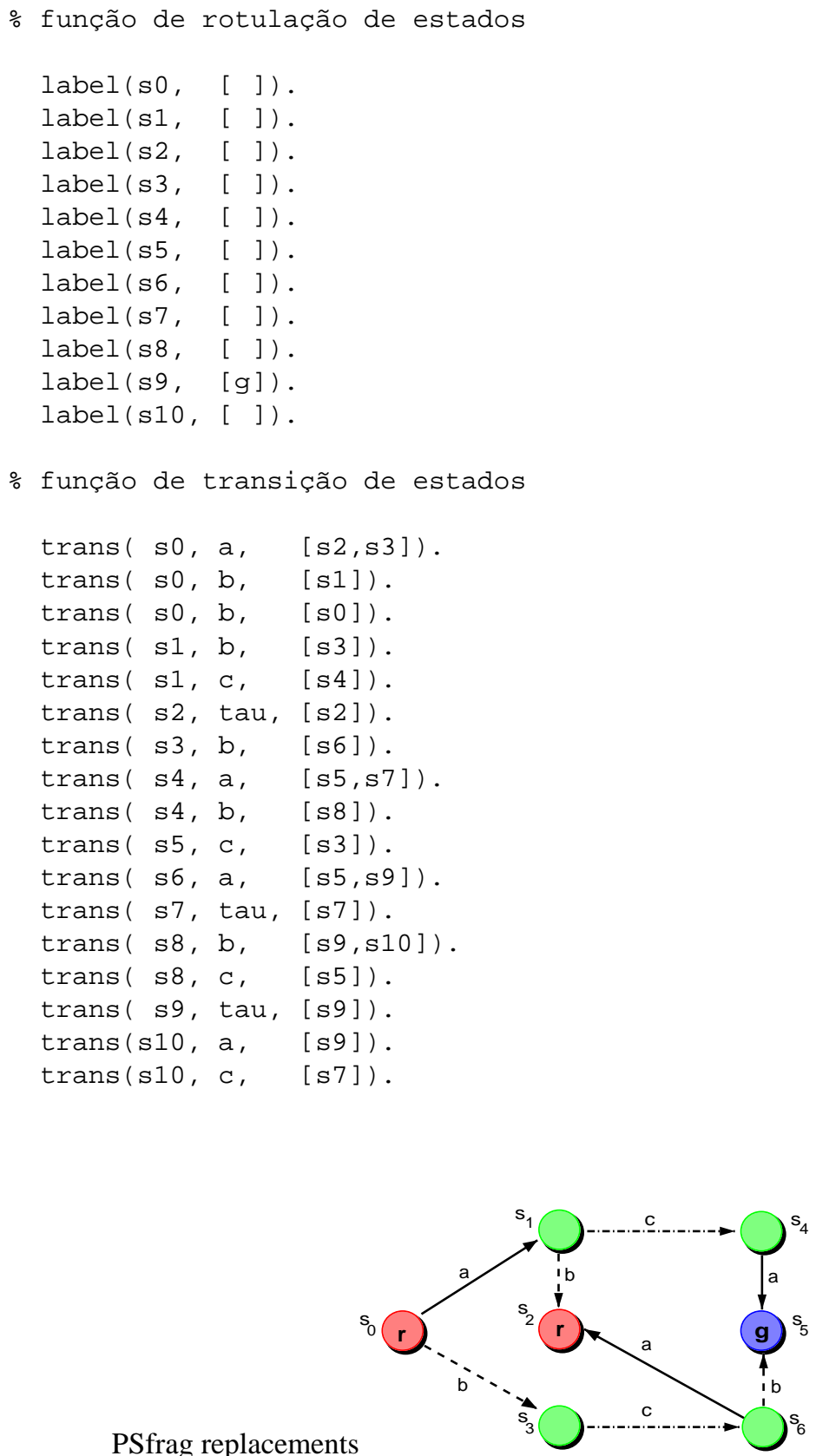

Figura B.2: Domínio de planejamento para meta de alcançabilidade estendida $\mathcal{D}^{2}$. 
Exemplo B.2. O modelo apresentado na Figura B.2 pode ser especificado conforme a seguir:

\% função de rotulação de estados

label (s0, [r]).

label (s1, [ ]).

label (s2, [r]).

label (s3, [ ]).

label (s4, [ ]).

label (s5, [g]).

label (s6, [ ]).

․ função de transição de estados

$\operatorname{trans}(\mathrm{s} 0, \mathrm{a}, \quad[\mathrm{s} 1])$.

$\operatorname{trans}(\mathrm{s} 0, \mathrm{~b}, \quad[\mathrm{~s} 3])$.

$\operatorname{trans}(\mathrm{s} 1, \mathrm{c}, \quad[\mathrm{s} 4])$.

$\operatorname{trans}(\mathrm{s} 1, \mathrm{~b}, \quad[\mathrm{~s} 2])$.

trans (s2, tau, [s2]).

$\operatorname{trans}(s 3, c, \quad[s 6])$.

$\operatorname{trans}(\mathrm{s} 4, \mathrm{a}, \quad[\mathrm{s} 5])$.

trans (s5, tau, [s5]).

$\operatorname{trans}(s 6, a, \quad[s 2])$.

$\operatorname{trans}(s 6, b, \quad[s 5])$.

\section{B.2 Verificador de modelos para $\alpha$-CTL}

A seguir, apresentamos um protótipo de verificador de modelos para $\alpha$-CTL. A notação para os operadores temporais usados nesse protótipo é apresentada na Tabela B.1.

\begin{tabular}{|c|c|c|c|c|c|c|c|c|c|c|c|}
\hline$\alpha$-CTL & $\neg$ & $\wedge$ & $\vee$ & $\exists \odot$ & $\forall \odot$ & $\exists \odot$ & $\forall \odot$ & $\exists \odot$ & $\forall \odot$ & $\exists \bullet$ & $\forall \bullet$ \\
\hline PROLOG & not & and & or & ex & ax & eg & ag & ef & af & eu & au \\
\hline
\end{tabular}

Tabela B.1: Operadores usados no verificador de modelos para $\alpha$-CTL.

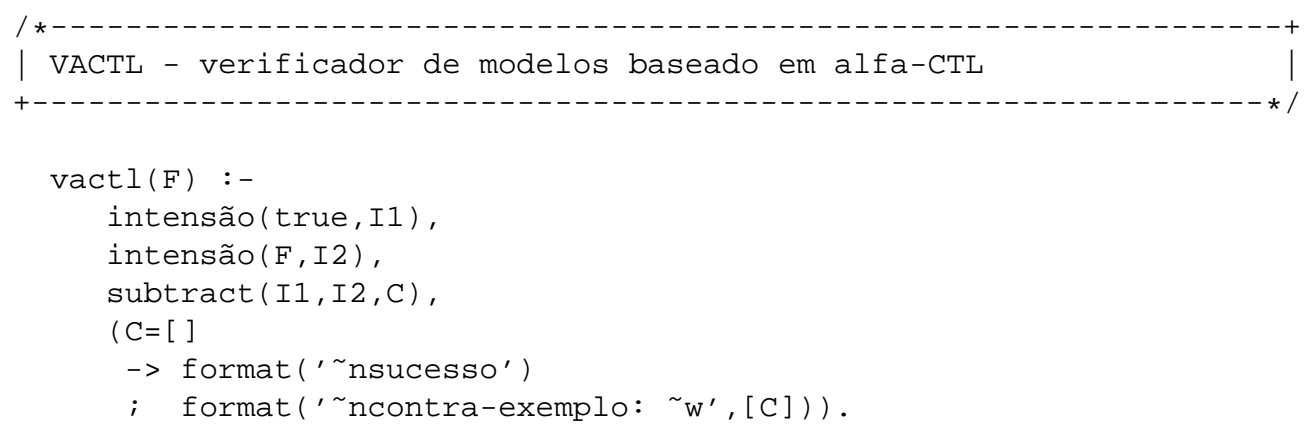


\% proposições atômicas

intensão $(P, I)$ :-

atomic $(P)$,

$\operatorname{set}(S, \operatorname{sat}(S, P), I)$.

o operadores proposicionais

intensão(not (P), I) :- !,

atomic (P),

intensão(true, I1),

intensão ( $P, I 2)$,

subtract (I1, I2, I) .

intensão (and $(F 1, F 2), I):-$ !,

intensão (F1, I1),

intensão (F2,I2),

intersection (I1, I2, I).

intensão $($ or $(F 1, F 2), I):-$ !,

intensão $(F 1, I 1)$,

intensão (F2, I2),

union (I1, I2, I).

- operadores temporais locais

intensão $(\operatorname{ex}(F), I):-$ !,

intensão $(F, I 1)$,

preImagemFraca (I1, I2),

union (I1, I2, I).

intensão $(\operatorname{ax}(F), I):-$ !,

intensão $(F, I 1)$,

preImagemForte (I1, I2),

union (I1, I2, I).

\% operadores temporais globais

intensão $(\operatorname{eg}(F), I):-$ !,

intensão ( $F, I 1)$,

pfmaxeg (nil, I1, I) .

intensão $(\operatorname{ag}(F), I)$ :- !,

intensão ( $F, I 1)$,

pfmaxAG (nil, I1, I) .

intensão(eu $(F 1, F 2), I)$ :- !,

intensão ( $F 1, I 1)$,

intensão (F2, I2),

pfminEU (I1, nil, I2, I) . 


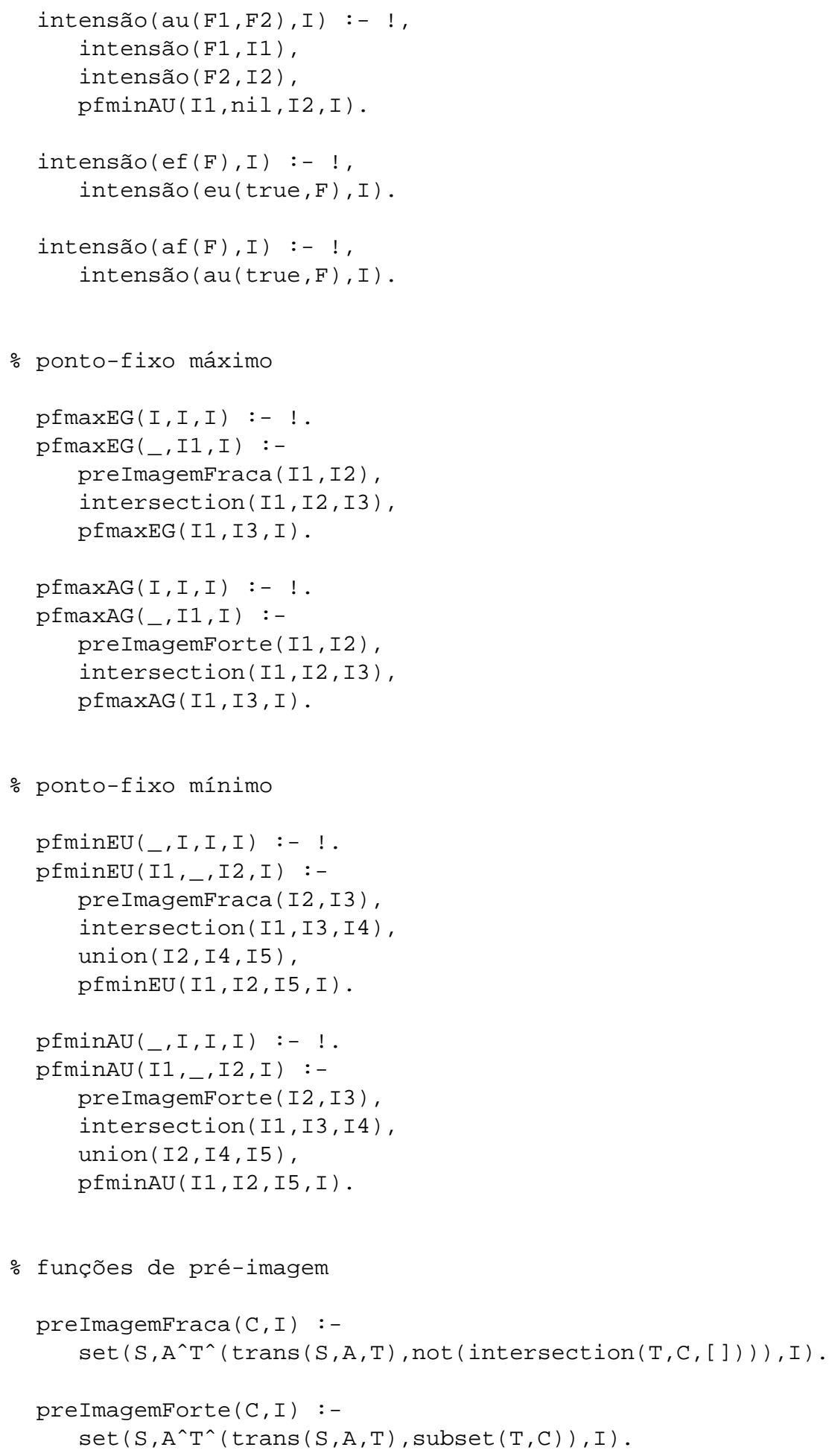




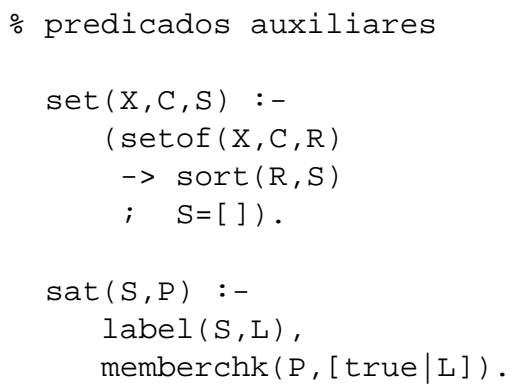

A seguir, apresentamos dois exemplos que mostram como podemos utilizar o verificador de modelos implementado pelo predicado vact $1 / 1$.

Exemplo B.3. Para verificar se o modelo apresentado na Figura B.1 satisfaz a propriedade $\forall \odot g$, basta digitar:

?- $\quad \operatorname{vactl}(\mathrm{af}(\mathrm{g}))$.

contra-exemplo: [s2, s3, s5, s6, s7]

Como resposta, o verificador apresenta o conjunto de estados a partir dos quais não é garantido alcançar um estado satisfazendo a propriedade $g$.

Exemplo B.4. Para verificar se o modelo apresentado na Figura B.2. satisfaz a propriedade $\forall((r \vee \forall \odot r \vee \forall \odot \forall \odot r) \sqcup g)$, basta digitar:

Como resposta, o verificador apresenta o conjunto de estados a partir dos quais não é garantido alcançar um estado satisfazendo a propriedade g, passando apenas por estados a partir dos quais é garantido alcançar um estado satisfazendo a propriedade r, em até dois passos. 


\section{B.3 Planejador baseado em $\alpha$-CTL}

A seguir, apresentamos um protótipo do planejador baseado em $\alpha$-CTL. A notação para os operadores temporais usados nesse protótipo é apresentada na Tabela B.1 (pág. 121).

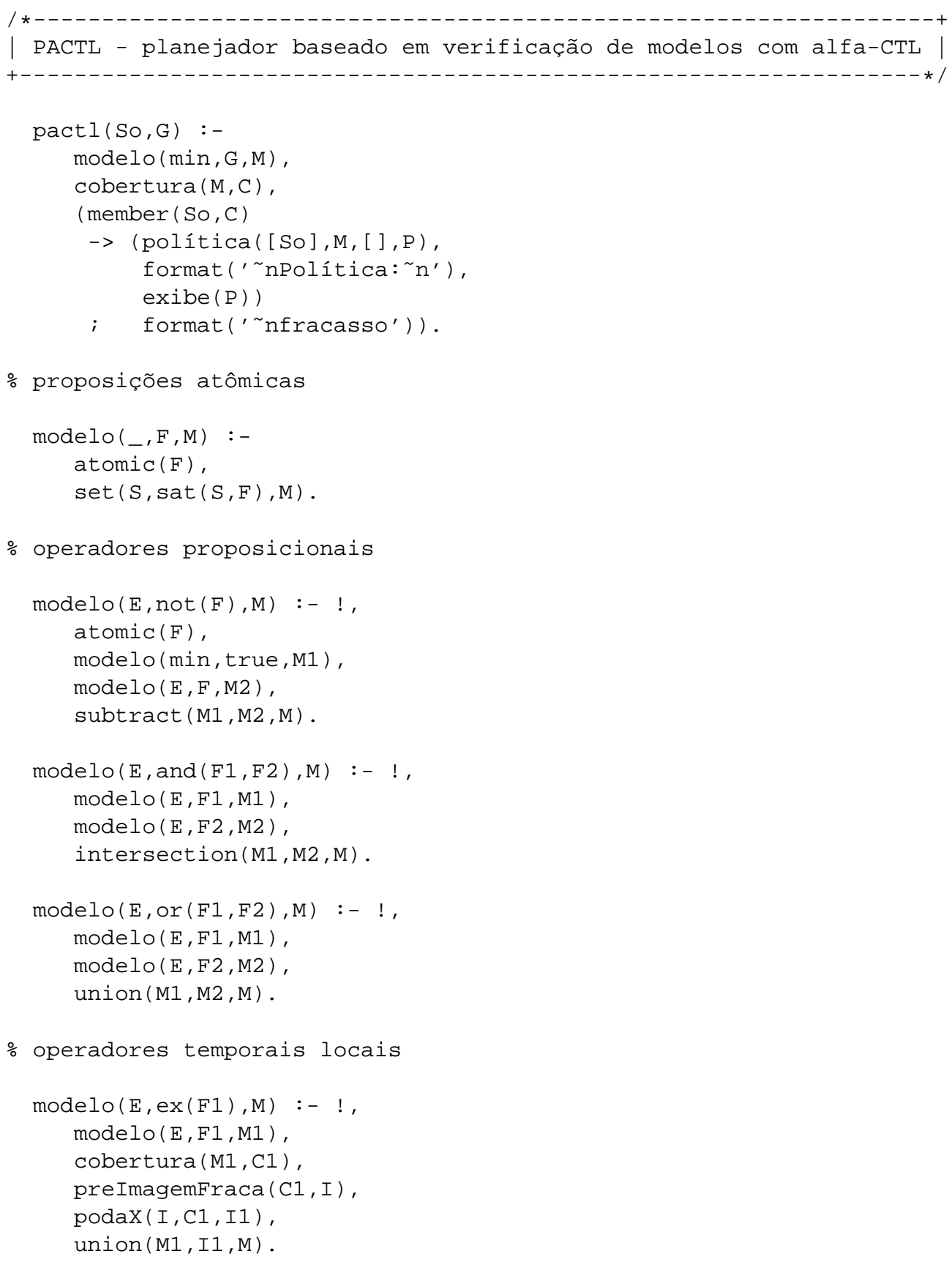




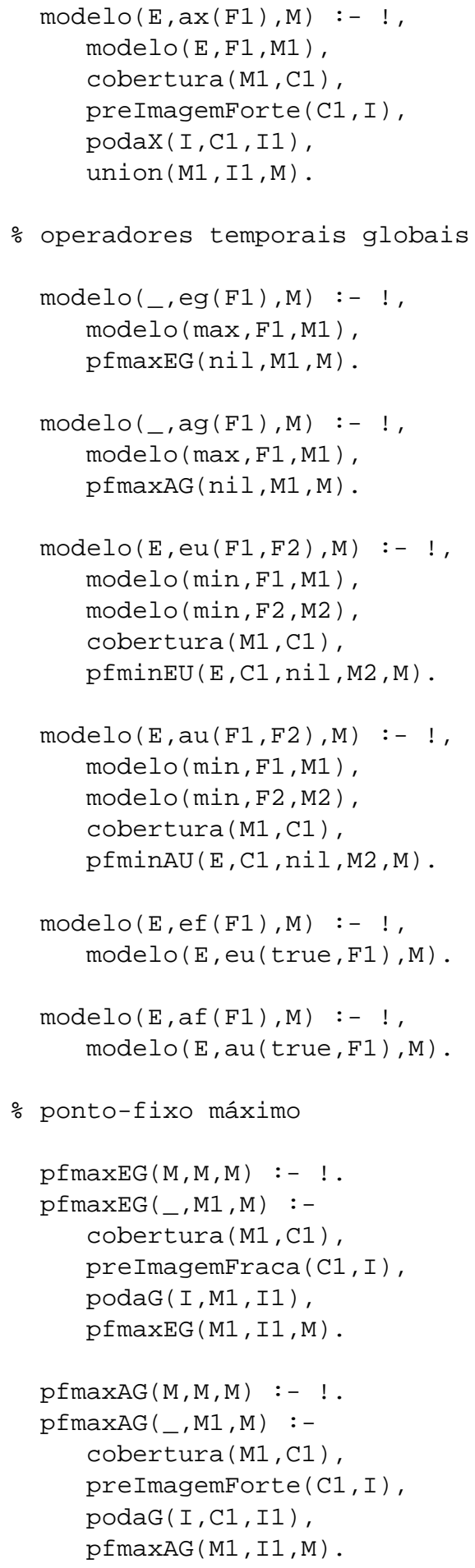




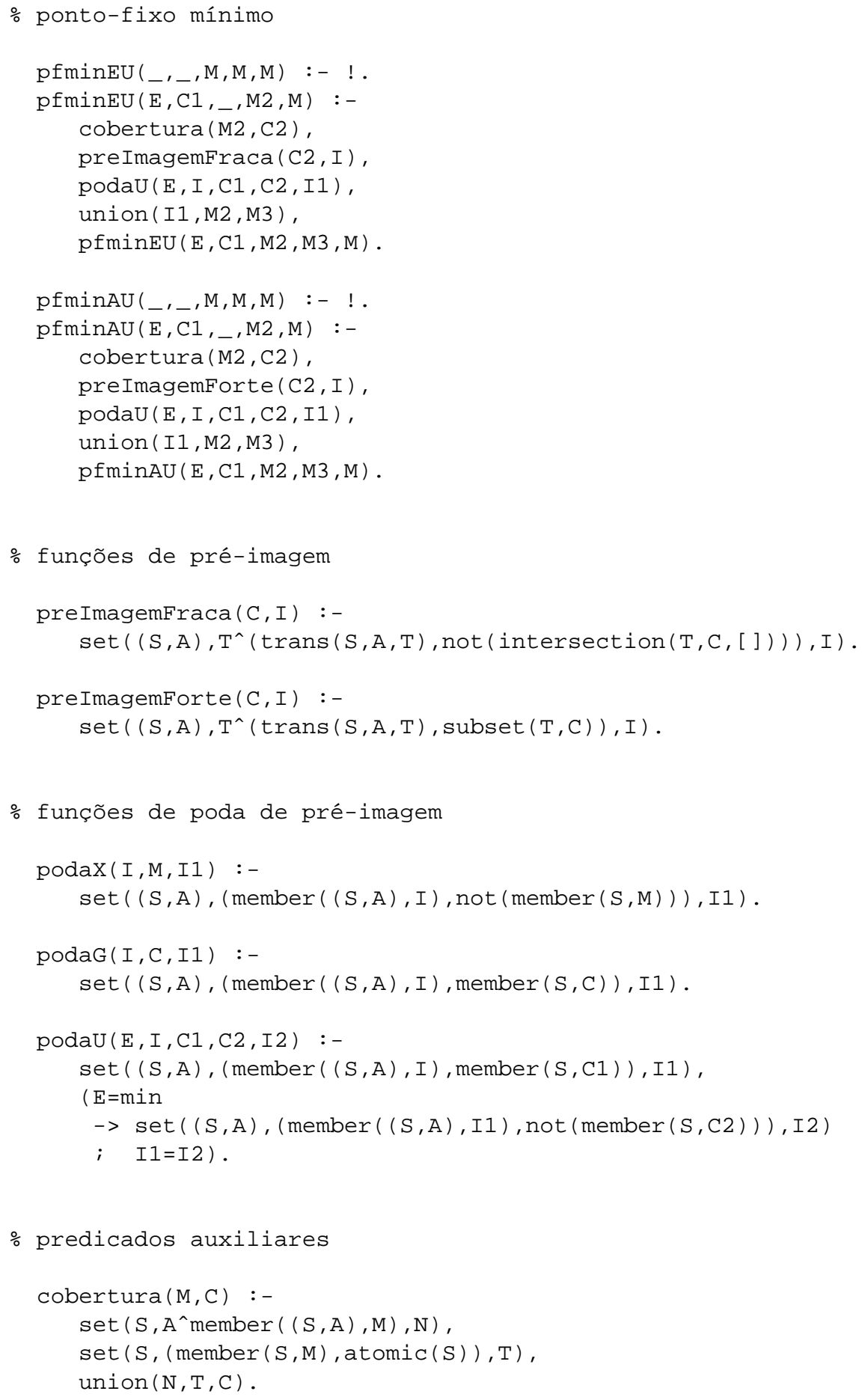




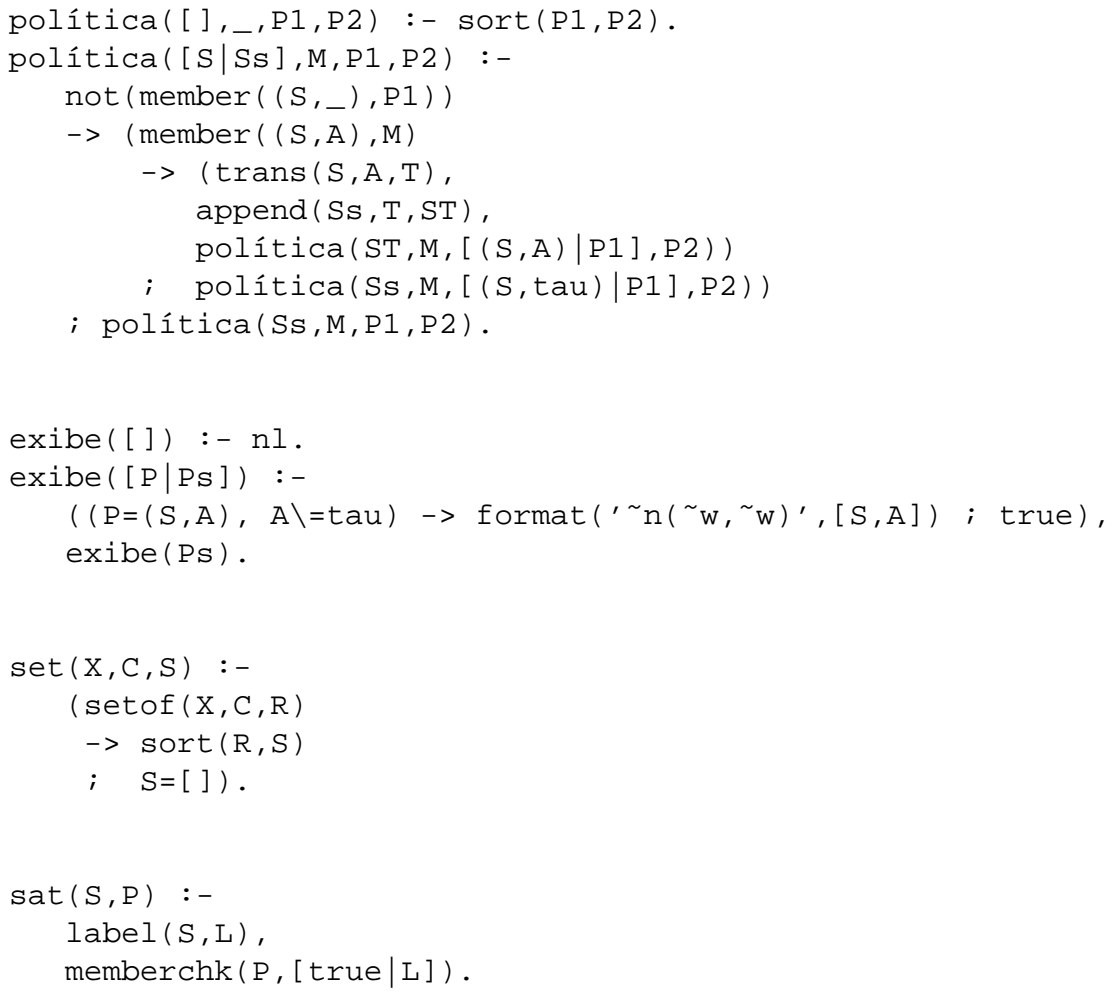

A seguir, apresentamos exemplos que mostram como podemos utilizar o planejador implementado pelo predicado pact $1 / 2$.

Exemplo B.5 (metas de alcançabilidade simples). Considere o domínio de planejamento apresentado na Figura B.1. Suponha que a meta de planejamento seja alcançar um estado satisfazendo a propriedade $g$, a partir do estado $s_{0}$.

Para obter uma solução fraca para esse problema $(\exists \bullet g)$, digite:

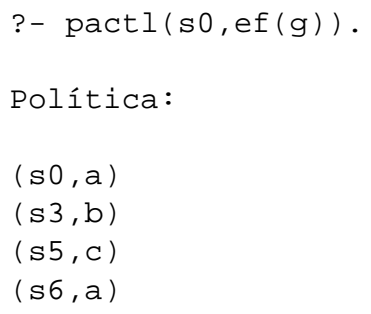


Para obter uma solução forte $(\forall \odot g)$, digite:

?- $\operatorname{pactl}(\mathrm{s} 0, \mathrm{af}(\mathrm{g}))$.

Política:

$(\mathrm{s} 0, \mathrm{~b})$

$(\mathrm{s} 1, \mathrm{c})$

$(s 10, a)$

$(\mathrm{s} 4, \mathrm{~b})$

$(\mathrm{s} 8, \mathrm{~b})$

Finalmente, para obter uma solução forte-cíclica $(\forall \bullet \exists \bullet g)$, digite:

?- pactl(s0,ag(ef(g))).

Política:

$(\mathrm{s} 0, \mathrm{~b})$

$(\mathrm{s} 1, \mathrm{~b})$

$(\mathrm{s} 3, \mathrm{~b})$

$(\mathrm{s} 5, \mathrm{c})$

$(s 6, a)$

Exemplo B.6 (meta de alcançabilidade estendida). Considere o domínio de planejamento apresentado na Figura B.2. e suponha que a meta de planejamento seja alcançar, garantidamente, um estado satisfazendo a propriedade g, passando apenas por estados a partir dos quais seja garantido alcançar um estado satisfazendo a propriedade $r$, em no máximo dois passos (i.e., $\forall((r \vee \forall \odot r \vee \forall \odot \forall \odot r) \sqcup g)$. Então, basta digitar:

?- $\operatorname{pactl}(\mathrm{s} 0, \mathrm{au}(\operatorname{or}(r, \operatorname{or}(\operatorname{ax}(r), \operatorname{ax}(\operatorname{ax}(r)))), g))$.

Política:

$(\mathrm{s} 0, \mathrm{~b})$

$(s 3, c)$

$(\mathrm{s} 6, \mathrm{~b})$ 


\section{Apêndice C}

\section{Teoria da decisão}

A disponibilidade de informação quantitativa sobre a incerteza que um agente tem a respeito do comportamento da natureza altera radicalmente o modo como ele toma suas decisões. Quando um agente racional toma decisões com base num modelo de incerteza puramente qualitativo, ele escolhe suas ações levando em conta apenas o fato dessas ações permitirem ou não que sua meta seja alcançada. Porém, quando um agente dispõe de um modelo de incerteza quantitativo, para tomar uma decisão, ele deve primeiro definir suas preferências entre os diferentes resultados das diversas ações possíveis e, depois, ponderar essas preferências levando em conta os graus de crença que ele tem a respeito da possibilidade de ocorrência de cada resultado. Em suma, quando dispomos de um modelo de incerteza quantitativo, uma estratégia racional de seleção de ações para o agente deve ser baseada na teoria da decisão [French, 1986], que combina teoria da probabilidade, para lidar com graus de crença, e teoria da utilidade, para lidar com preferências.

\section{C.1 Teoria da probabilidade}

Um formalismo para descrever graus de crença deve ser capaz de tratar dois aspectos importantes: (i) a representação de sentenças, às quais são atribuídos os graus de crença; e (ii) a representação de dependências dos graus de crença em relação à experiência do agente. Nessa subseção, apresentamos um formalismo para descrição de graus de crença que é baseado em [Russell \& Norvig, 2002, Seção 13.2]. Nesse formalismo, usamos uma extensão da lógica proposicional para representar sentenças; e declarações de probabilidade (in)condicional para representar dependências dos graus de crença em relação à experiência do agente. 
Proposições. Uma variável aleatória representa o resultado, inicialmente desconhecido, de um determinado experimento. Cada variável aleatória $X$ tem um domínio $\Omega_{X}$, denominado espaço amostral de $X$. Elementos de um espaço amostral são denominados estados elementares. Assumimos que os estados elementares de um espaço amostral são mutuamente exclusivos e exaustivos e, portanto, cada experimento deve ter exatamente um resultado.

Uma proposição atômica é uma expressão da forma $X=\omega$, onde $X$ é uma variável aleatória e $\omega \in \Omega_{X}$ é um estado elementar no espaço amostral de $X$. Uma proposição composta, ou simplesmente proposição, é uma combinação lógica de outras proposições atômicas ou compostas, e.g., $(X=a) \wedge((Y=b) \vee(Y=c))$.

Exemplo C.1 (Lançamento de uma moeda). Ao lançarmos uma moeda, há dois resultados possíveis: cara ou coroa. Então, se X é uma variável aleatória representando o resultado desse experimento, seu espaço amostral é $\Omega_{X}=\{$ cara, coroa $\}$. Ademais, como os estados elementares num espaço amostral são mutuamente exclusivos e exaustivos, segue que a proposição $(X=$ cara $\wedge X=$ coroa $)$ é contraditória e que a proposição $(X=$ cara $\vee X=$ coroa $)$ é válida.

Medidas de probabilidade. Uma medida de probabilidade é uma função $\mathcal{P}$, que associa a cada proposição um grau de crença entre 0 e 1 . Um grau de crença definido a priori, na ausência de qualquer outra evidência, é denominado probabilidade incondicional. A probabilidade incondicional associada a uma proposição $a$ é denotada por $P(a)$. Quando obtemos alguma evidência relativa a variáveis aleatórias anteriormente desconhecidas, em vez de probabilidades incondicionais, devemos usar probabilidades condicionais. A probabilidade condicional associada a uma proposição $a$, dado que conhecemos a evidência $b$, é denotada por $\mathcal{P}(a \mid b)$. Probabilidades condicionais podem ser definidas em termos de probabilidades incondicionais, como a seguir:

$$
\mathcal{P}(a \mid b)=\frac{\mathcal{P}(a \wedge b)}{\mathcal{P}(b)}, \text { sendo } \mathcal{P}(b) \neq 0 .
$$

Axiomas de probabilidade. Os axiomas de probabilidade definem a semântica das declarações de probabilidade. Como as probabilidades condicionais podem ser definidas em termos de probabilidades incondicionais (vide Equação C.1), os axiomas básicos de probabilidade (também denominados axiomas de Kolmogorov) são definidos apenas para declarações de probabilidade incondicional: 
1. Toda proposição $a$ tem uma probabilidade entre 0 e 1 ; ou seja, $0 \leq \mathcal{P}(a) \leq 1$.

2. Proposições contraditórias têm probabilidade 0 , e proposições válidas têm probabilidade 1; ou seja, $\mathcal{P}(\perp)=0$ e $P(T)=1$.

3. A probabilidade de uma disjunção é dada por: $\mathscr{P}(a \vee b)=\mathscr{P}(a)+\mathscr{P}(b)-\mathscr{P}(a \wedge b)$.

Exemplo C.2 (Regra da negação). Podemos derivar uma série de fatos úteis a partir dos axiomas básicos de probabilidade. Por exemplo, a regra da negação pode ser derivada da seguinte forma:

$$
\begin{aligned}
\mathcal{P}(a \vee \neg a) & =\mathcal{P}(a)+\mathcal{P}(\neg a)-\mathcal{P}(a \wedge \neg a) & & \text { (pelo axioma 3, substituindo b por } \neg a) \\
\mathcal{P}(\top) & =\mathcal{P}(a)+\mathcal{P}(\neg a)-\mathcal{P}(\perp) & & \text { (por equivalência lógica) } \\
1 & =\mathcal{P}(a)+\mathcal{P}(\neg a) & & \text { (pelo axioma 2) } \\
\mathcal{P}(\neg a) & =1-\mathcal{P}(a) & & \text { (por álgebra) }
\end{aligned}
$$

Os axiomas de probabilidade também implicam que toda distribuição de probabilidades sobre uma determinada variável aleatória $X$ deve somar 1 , ou seja:

$$
\sum_{\omega \in \Omega_{X}} \mathcal{P}(X=\omega)=1
$$

\section{C.2 Teoria da utilidade}

A teoria da utilidade [von Neumann \& Morgenstern, 1944] proporciona um método para escolhas em contextos onde todas as possíveis alternativas podem ser enumeradas. Segundo essa teoria, as preferências de um agente sobre os resultados de suas ações são captadas por uma função de utilidade, que atribui um único número para expressar a desejabilidade de cada resultado. Essas utilidades são combinadas com as probabilidades de ocorrência dos resultados das ações, para fornecerem uma utilidade esperada referente a cada ação.

Relação de preferência. Uma estrutura de preferência pode ser formulada como uma relação de preferência binária. Seja $\Omega$ o conjunto de todos os efeitos resultantes das decisões de um agente. Assumimos que a estrutura de preferência do agente sobre $\Omega$ é capturada pela relação de preferência $>$ sobre $\Omega$, definida como uma relação binária com a seguinte propriedade: para todo par de resultados $\omega, \omega^{\prime} \in \Omega$,

- $\omega$ é preferível $a \omega^{\prime}$ se e somente se $\omega>\omega^{\prime}$. 
Por conveniência, também definimos mais duas outras relações binárias $\sim$ e $\gtrsim$ como segue: para todo par de resultados $\omega, \omega^{\prime} \in \Omega$,

- $\omega \sim \omega^{\prime}$ se e somente se $\omega \ngtr \omega^{\prime}$ e $\omega^{\prime} \nsucc \omega$;

- $\omega \gtrsim \omega^{\prime}$ se e somente se $\omega>\omega^{\prime}$ ou $\omega \sim \omega^{\prime}$.

A relação de preferência $\gtrsim$ é uma relação de ordem total sobre $\Omega$, ou seja, quaisquer dois resultados $\omega, \omega^{\prime} \in \Omega$ são comparáveis. Formalmente, a relação de preferência induzida por $>\mathrm{e} \sim$ é uma relação binária com as seguintes propriedades: para todo $\omega, \omega^{\prime}, \omega^{\prime \prime} \in \Omega$,

1. Reflexividade: $\omega \sim \omega$.

2. Ordenabilidade: $\left(\omega>\omega^{\prime}\right) \vee\left(\omega^{\prime}>\omega\right) \vee\left(\omega \sim \omega^{\prime}\right)$.

3. Transitividade: $\left(\omega>\omega^{\prime}\right) \wedge\left(\omega^{\prime}>\omega^{\prime \prime}\right) \Rightarrow\left(\omega>\omega^{\prime \prime}\right)$.

Essas três propriedades, conhecidas como axiomas básicos de utilidade, especificam as restrições semânticas óbvias sobre preferências. As duas primeiras propriedades estabelecem que, dados dois resultados quaisquer, o agente deve preferir um ao outro ou, então, classificá-los como igualmente preferíveis (mas não pode simplesmente evitar uma decisão entre eles). A terceira propriedade estabelece que a estrutura de preferências de um agente deve ser transitiva; do contrário ele poderá agir de forma irracional.

Exemplo C.3 (Violação da transitividade). Suponha que um agente tenha preferências não transitivas $\omega>\omega^{\prime}>\omega^{\prime \prime}>\omega$ (Figura C.1). Se inicialmente o agente possui $\omega$, podemos oferecer-lhe $\omega^{\prime \prime}$ em troca de $\omega$ e mais alguma quantia em dinheiro. Como o agente prefere $\omega^{\prime \prime}$, ele aceita fazer essa troca. Em seguida, podemos oferecer $\omega^{\prime}$ em troca de $\omega^{\prime \prime}$ e extrair mais outra quantia do agente. Finalmente, oferecemos $\omega$ em troca de $\omega^{\prime}$. Isso nos leva de volta à situação inicial, exceto pelo fato de que agora o agente tem menos dinheiro.

Preferências sobre loterias. Estruturas de preferência podem ser estendidas para efeitos incertos. Efeitos incertos podem ser representados como distribuições de probabilidades discretas sobre $\Omega$, denominadas loterias. Uma loteria $\lambda$ com resultados possíveis $\lambda_{1}, \ldots, \lambda_{n}$, que podem ocorrer com probabilidades $\mathcal{P}\left(\lambda_{1}\right)=p_{1}, \ldots, \mathcal{P}\left(\lambda_{n}\right)=p_{n}$, é escrita como:

$$
\lambda=\left[p_{1}, \lambda_{1} ; \ldots ; p_{n}, \lambda_{n}\right], \text { onde } \sum_{i=1}^{n} p_{i}=1 .
$$

Em geral, cada resultado de uma loteria pode ser um resultado atômico ou uma outra loteria. Uma loteria degenerada, com apenas um resultado possível $\omega$, pode ser escrita como $\omega$ ou $[1, \omega]$. 


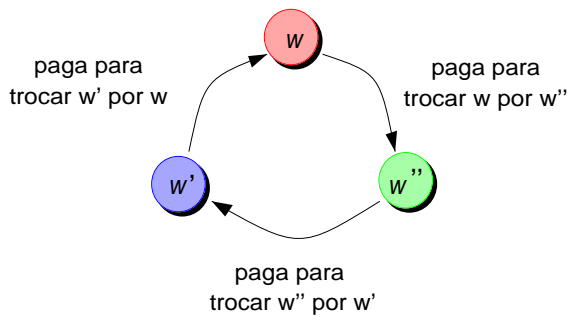

Figura C.1: Comportamento irracional gerado pela violação à propriedade de transitividade.

Exemplo C.4 (Ações como loterias). Considere a situação ilustrada na Figura C.2. Quando o agente tenta entrar-no-elevador, pode ser que a porta automática se feche antes que ele consiga ter êxito (com probabilidade 0.2); do contrário, ele consegue chegar ao térreo. Por outro lado, quando o agente escolhe ir-pela-escada, ele sempre consegue chegar ao térreo. Em termos de planejamento, a ação entrar-no-elevador pode ser vista como a loteria [0.2, saguao; 0.8 , terreo]; enquanto a ação ir-pela-escada pode ser vista como a loteria degenerada $[1$, terreo].

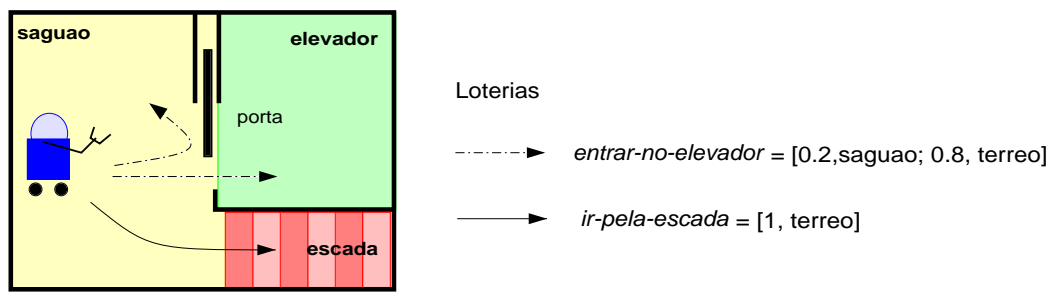

Figura C.2: Ações com efeitos incertos vistas como loterias.

A principal questão para a teoria da utilidade é compreender como as preferências entre loterias complexas estão relacionadas a preferências entre os resultados subjacentes nessas loterias. Para tanto, precisamos impor restrições sobre a relação de preferência entre loterias, de modo semelhante àquele como impusemos restrições sobre os graus de crença, a fim de obter os axiomas de probabilidade (vide Subseção C.1).

Seja $\Lambda(\Omega)$ o conjunto de todas as loterias sobre o conjunto de resultados $\Omega$. Estruturas de preferências sobre loterias devem ter propriedades adicionais, que são consistentes com a intuição humana sobre estruturas envolvendo incerteza [Russell \& Norvig, 2002]. Sejam três loterias $\lambda, \lambda^{\prime}, \lambda^{\prime \prime} \in \Lambda(\Omega)$ : 
1. Continuidade: se $\lambda^{\prime}$ estiver entre $\lambda$ e $\lambda^{\prime \prime}$ em preferência, então há alguma probabilidade $p$ de que o agente fique indiferente entre escolher $\lambda^{\prime}$ por garantia ou escolher a loteria que produz $\lambda$ com probabilidade $p$ e $\lambda^{\prime \prime}$ com probabilidade $1-p$ :

$\lambda>\lambda^{\prime}>\lambda^{\prime \prime} \Rightarrow \lambda^{\prime} \sim \exists p\left[p, \lambda ; 1-p, \lambda^{\prime \prime}\right]$.

2. Substitutibilidade: se um agente está indiferente entre $\lambda$ e $\lambda^{\prime}$, então o agente está indiferente entre outras duas loterias complexas que são a mesma loteria, exceto pelo fato de $\lambda$ ser substituída por $\lambda^{\prime}$ em uma delas:

$\lambda \sim \lambda^{\prime} \Rightarrow\left[p, \lambda ; 1-p, \lambda^{\prime \prime}\right] \sim\left[p, \lambda^{\prime} ; 1-p, \lambda^{\prime \prime}\right]$.

3. Monotonicidade: suponha que existam duas loterias que tenham os mesmos dois resultados, $\lambda$ e $\lambda^{\prime}$. Se um agente prefere $\lambda$ a $\lambda^{\prime}$, então o agente deve preferir a loteria que tem uma probabilidade mais alta para $\lambda$ :

$\lambda>\lambda^{\prime} \Rightarrow\left(p \geq q \Leftrightarrow\left[p, \lambda ; 1-p, \lambda^{\prime}\right] \gtrsim\left[q, \lambda ; 1-q, \lambda^{\prime}\right]\right)$.

4. Decomponibilidade: loterias complexas podem ser reduzidas a loterias simples com o uso das leis da probabilidade:

$$
\left[p, \lambda ; 1-p,\left[q, \lambda^{\prime} ; 1-q, \lambda^{\prime \prime}\right]\right] \sim\left[p, \lambda ;(1-p) q, \lambda^{\prime} ;(1-p)(1-q), \lambda^{\prime \prime}\right] .
$$

Essas propriedades, conhecidas como axiomas estendidos de utilidade, implicam que a relação de preferência estendida para loterias é uma ordem total sobre $\Lambda(\Omega)$.

Princípio da utilidade. Se a relação de preferência estendida satisfaz os sete axiomas de utilidade apresentados nessa subseção, então existe uma função de utilidade $u: \Lambda(\Omega) \mapsto \mathbb{R}$, tal que, para todo par de loterias $\lambda, \lambda^{\prime} \in \Lambda(\Omega)$ :

- $u(\lambda)>u\left(\lambda^{\prime}\right)$ se e somente se $\lambda>\lambda^{\prime}$;

- $u(\lambda)=u\left(\lambda^{\prime}\right)$ se e somente se $\lambda \sim \lambda^{\prime}$.

A utilidade esperada de uma loteria $\lambda=\left[p_{1}, \lambda_{1} ; \ldots ; p_{n}, \lambda_{n}\right]$ é o somatório do produto da probabilidade $p_{i}$ de cada resultado $\lambda_{i}$ pela utilidade $u\left(\lambda_{i}\right)$ desse resultado:

$$
u(\lambda)=u\left(\left[p_{1}, \lambda_{1} ; \ldots ; p_{n}, \lambda_{n}\right]\right)=\sum_{i=1}^{n} p_{i} . u\left(\lambda_{i}\right)
$$

Assim, a função de utilidade pode ser definida apenas para elementos de $\Omega$; e sua definição sobre $\Lambda(\Omega)$ pode ser obtida usando a Equação (C.4). Ademais, a função de utilidade é única, a 
menos de uma transformação linear, ou seja, $u_{1}($.$) e u_{2}()=.a . u_{1}()+$.$b , para a>0$, são funções de utilidade correspondentes à mesma estrutura de preferências.

Tomada de decisão. Dado um conjunto finito de loterias $\Lambda(\Omega)$, um agente racional irá escolher a loteria que é maximal em $\Lambda(\Omega)$, ou seja, a loteria que maximiza a utilidade esperada de todos os resultados possíveis. Sob esse princípio, um problema de decisão entre loterias pode ser resolvido com a ajuda de uma função de utilidade: o agente encontra a utilidade máxima esperada:

$$
u^{\star}=\max _{\lambda \in \Lambda(\Omega)} u(\lambda)
$$

e, então, toma uma decisão ótima escolhendo uma loteria cuja utilidade máxima esperada é $u^{\star}$ :

$$
\lambda^{\star}=\arg \max _{\lambda \in \Lambda(\Omega)} u(\lambda)
$$

É importante salientar que a existência de uma função de utilidade, que descreve as preferências de um agente racional sob algum aspecto, não significa necessariamente que esse agente deva maximizar explicitamente essa função em suas deliberações. O comportamento racional pode depender de vários outros fatores, alguns dos quais podem ser mais importantes que apenas a maximização da utilidade explícita [Russell \& Norvig, 2002]. Por exemplo, podem existir diversas sequiências de ações que levem um agente a atingir sua meta: algumas mais rápidas, outras mais seguras, outras mais confiáveis ou, ainda, mais econômicas; maximizar um desses aspectos em detrimento de outros pode não ser a decisão mais racional para um certo agente. 


\section{Referências Bibliográficas}

[Altman, 1999] Altman, E. (1999). Constrained Markov decision processes. Chapman \& Hall, Florida.

[Andersen, 1997] Andersen, H. R. (1997). Introduction to binary decision diagrams. In http://www.itu.dk/people/hra/notes-index.html. (acessado em 08/10/2007).

[Bacchus \& Kabanza, 1996] Bacchus, F. \& Kabanza, F. (1996). Planning for temporally extended goals. In $A A A I^{\prime} 96$, pp. 1215-1222. AAAI Press.

[Baral \& Zhao, 2004] Baral, C. \& Zhao, J. (2004). Goal specification in presence of nondeterministic actions. In de Mántaras, R. L. e Saitta, L., ECAI, pp. 273-277, Valencia, Spain. IOS Press.

[Baral \& Zhao, 2006] Baral, C. \& Zhao, J. (2006). Goal specification, non-determinism and quantifying over policies. In AAAI-06, Boston, Massachusetts, USA. AAAI Press.

[Barto et al., 1993] Barto, A. G., Bradtke, S. J., \& Singh, S. P. (1993). Learning to act using real-time dynamic programming. UM-CS-1993-002.

[Bäckström, 1995] Bäckström, C. (1995). Expressive equivalence of planning formalisms. Artificial Intelligence, 76 (1-2):17-34.

[Bellman, 1957] Bellman, R. E. (1957). Dynamic programming. Princeton University Press, USA.

[Bertoli et al., 2001] Bertoli, P., Cimatti, A., Pistore, M., Roveri, M., \& Traverso, P. (2001). MBP: a model based planner. In IJCAI-01, Seatle.

[Bertoli \& Pistore, 2004] Bertoli, P. \& Pistore, M. (2004). Planning with extended goals and partial observability. In ICAPS, pp. 270-278. 
[Bertsekas \& Tsitsiklis, 1991] Bertsekas, D. P. \& Tsitsiklis, J. N. (1991). An analysis of stochastic shortest path problems. Math. Oper. Res., 16(3):580-595.

[Blackwell \& Girshik, 1979] Blackwell, D. \& Girshik, M. A. (1979). Theory of games and statistical decisions. Dover, New York.

[Bonet \& Geffner, 2003a] Bonet, B. \& Geffner, H. (2003a). Faster heuristic search algorithms for planning with uncertainty and full feedback. In IJCAI-03, pp. 1233-1238.

[Bonet \& Geffner, 2003b] Bonet, B. \& Geffner, H. (2003b). Labeled RTDP: Improving the convergence of real-time dynamic programming. In ICAPS-03.

[Bonet \& Geffner, 2005] Bonet, B. \& Geffner, H. (2005). mGPT: A probabilistic planner based on heuristic search. In JAIR, volume 24, pp. 933-944.

[Boutilier et al., 1999] Boutilier, C., Dean, T., \& Hanks, S. (1999). Decision-theoretic planning: structural assumptions and computational leverage. Journal of Artificial Intelligence Research, 11:1-94.

[Brafman \& Hoffmann, 2004] Brafman, R. I. \& Hoffmann, J. (2004). Conformant planning via heuristic forward search: a new approach. In ICAPS, pp. 355-364.

[Bryant, 1986] Bryant, R. E. (1986). Graph-based algorithms for Boolean function manipulation. IEEE Transactions on Computers, 35(8):677-691.

[Bryant, 1992] Bryant, R. E. (1992). Symbolic Boolean manipulation with ordered binarydecision diagrams. ACM Computing Surveys, 24(3):293-318.

[Bryce \& Kambhampati, 2004] Bryce, D. \& Kambhampati, S. (2004). Heuristic guidance measures for conformant planning. In ICAPS, pp. 365-375.

[Burch et al., 1990] Burch, J. R., Clarke, E. M., McMillan, K., Dill, D., \& Hwang, L. (1990). Symbolic Model Checking: $10^{20}$ States and Beyond. In Proceedings of the Fifth Annual IEEE Symposium on Logic in Computer Science, pp. 1-33, Washington, D.C. IEEE Computer Society Press.

[Bylander, 1994] Bylander, T. (1994). The computational complexity of propositional STRIPS planning. Artificial Intelligence, 69(1-2):165-204. 
[Cimatti et al., 1997] Cimatti, A., Giunchiglia, F., Giunchiglia, E., \& Traverso, P. (1997). Planning via model checking: A decision procedure for $\mathcal{A R}$. In $E C P$, pp. 130-142.

[Cimatti et al., 1998] Cimatti, A., Roveri, M., \& Traverso, P. (1998). Strong planning in nondeterministic domains via model checking. In Artificial Intelligence Planning Systems, pp. 36-43.

[Clarke \& Emerson, 1982] Clarke, E. M. \& Emerson, E. A. (1982). Design and synthesis of synchronization skeletons using branching-time temporal logic. In Logic of Programs, Workshop, pp. 52-71, London, UK. Springer-Verlag.

[Clarke \& Wing, 1996] Clarke, E. M. \& Wing, J. (1996). Formal methods: state of the art and future directions. In ACM Computing Systems Surveys, volume 28(4), pp. 623-643, US. CMU.

[Cohen et al., 1989] Cohen, P. R., Greenberg, M. L., Hart, D. M., \& Howe, A. E. (1989). Trial by fire: understanding the design requirements for agents in complex environments. AI Mag., $10(3): 34-48$.

[Cormen et al., 2001] Cormen, T. H., Leiserson, C. E., Rivest, R. L., \& Stein, C. (2001). Introduction to algorithms. MIT Press e McGraw-Hill, Cambridge, MA, USA.

[Dai \& Goldsmith, 2007] Dai, P. \& Goldsmith, J. (2007). Topological value iteration algorithm for Markov decision processes. In Veloso, M. M., editor, IJCAI, pp. 1860-1865.

[Daniele et al., 1999] Daniele, M., Traverso, P., \& Vardi, M. Y. (1999). Strong cyclic planning revisited. In ECP, pp. 35-48.

[Dolgov \& Durfee, 2005] Dolgov, D. A. \& Durfee, E. H. (2005). Stationary deterministic policies for constrained MDPs with multiple rewards, costs, and discount factors. In IJCAI, pp. 1326-1331.

[Emerson, 1990] Emerson, E. A. (1990). Temporal and modal logic. In Handbook of Theoretical Computer Science, Volume B: Formal Models and Semantics (B), pp. 995-1072. .

[Emerson \& Halpern, 1986] Emerson, E. A. \& Halpern, J. Y. (1986). "Sometimes" and "not never" revisited: on branching versus linear time temporal logic. In Journal of the ACM, volume 33(1), pp. 151-178. 
[Fikes \& Nilsson, 1990] Fikes, R. E. \& Nilsson, N. J. (1990). STRIPS: A new approach to the application of theorem proving to problem solving. In Allen, J., Hendler, J., \& Tate, A., Readings in Planning, pp. 88-97. Kaufmann, San Mateo, CA.

[French, 1986] French, S. (1986). Decision theory: an introduction to the mathematics of rationality. Halsted Press, New York, NY, USA.

[Ghallab et al., 2004] Ghallab, M., Nau, D., \& Traverso, P. (2004). Automated planning: theory and practice. Morgan Kaufmann Publishers Inc., USA.

[Giunchiglia et al., 1997] Giunchiglia, E., Kartha, G. N., \& Lifschitz, V. (1997). Representing action: indeterminacy and ramifications. Artificial Intelligence, 95(2):409-438.

[Giunchiglia \& Traverso, 1999] Giunchiglia, F. \& Traverso, P. (1999). Planning as model checking. In $E C P$, pp. 1-20.

[Goodwin et al., 2002] Goodwin, R., Akkiraju, R., \& Wu, F. (2002). A decision-support system for quote generation. In Eighteenth national conference on Artificial intelligence, pp. 830837, Menlo Park, CA, USA. American Association for Artificial Intelligence.

[Hansen \& Zilberstein, 2001] Hansen, E. A. \& Zilberstein, S. (2001). LAO^ ${ }^{\star}$ a heuristic search algorithm that finds solutions with loops. In JAI, volume 29, pp. 35-62.

[Jensen et al., 2001] Jensen, R. M., Veloso, M. M., \& Bowling, M. H. (2001). OBDD-based optimistic and strong cyclic adversarial planning. In Proceedings of the 6th European Conference on Planning (ECP-O1).

[Jensen et al., 2004] Jensen, R. M., Veloso, M. M., \& Bryant, R. E. (2004). Fault tolerant planning: toward probabilistic uncertainty models in symbolic non-deterministic planning. In ICAPS, pp. 335-344.

[Kabanza et al., 1997] Kabanza, F., Barbeau, M., \& St.-Denis, R. (1997). Planning control rules for reactive agents. Artificial Intelligence, 95(1):67-11.

[Korf, 1987] Korf, R. E. (1987). Planning as search: a quantitative approach. Artificial Intelligence, 33:65-88.

[Kripke, 1963] Kripke, S. (1963). Semantical considerations on modal logic. Acta Philosophica Fennica, 16:83-94. 
[Krulwich, 1992] Krulwich, B. (1992). Planning for soft goals. In Proceedings of the first international conference on Artificial intelligence planning systems, pp. 289-290, San Francisco, CA, USA. Morgan Kaufmann Publishers Inc.

[Kushmerick et al., 1994] Kushmerick, N., Hanks, S., \& Weld, D. (1994). An algorithm for probabilistic least-commitment planning. In Proceedings of the Twelfth National Conference on Artificial Intelligence (AAAI-94), volume 2, pp. 1073-1078, Seattle, Washington, USA. AAAI Press/MIT Press.

[Lago et al., 2002] Lago, U. D., Pistore, M., \& Traverso, P. (2002). Planning with a language for extended goals. In Eighteenth national conference on Artificial intelligence, pp. 447-454, Menlo Park, CA, USA. American Association for Artificial Intelligence.

[LaValle, 2006] LaValle, S. M. (2006). Planning algorithms. Cambridge University Press, Cambridge, U.K. (disponível em http://planning.cs.uiuc.edu/).

[Levesque et al., 1997] Levesque, H. J., Reiter, R., Lesperance, Y., Lin, F., \& Scherl, R. B. (1997). GOLOG: A logic programming language for dynamic domains. Journal of Logic Programming, 31(1-3):59-83.

[Littman et al., 1995] Littman, M. L., Dean, T., \& Kaelbling, L. P. (1995). On the complexity of solving markov decision problems. In UAI-95, pp. 394-402, Providence, Rhode Island. AAAI Press / MIT Press.

[Liu \& Koenig, 2005] Liu, Y. \& Koenig, S. (2005). Existence and finiteness conditions for risksensitive planning: Results and conjectures. In Proceedings of the 21th Annual Conference on Uncertainty in Artificial Intelligence (UAI-05), pp. 354-36, Arlington, Virginia. AUAI Press.

[Markov, 1971] Markov, A. (1971). Extension of the limit theorems of probability theory to a sum of variables connected in a chain. In Dynamic Probabilistic Systems, volume 1, New York, USA. John Wiley \& Sons.

[Martelli \& Montanari, 1973] Martelli, A. \& Montanari, U. (1973). Additive AND/OR graphs. In IJCAI, pp. 1-11, Stanford, CA.

[Mausam et al., 2007] Mausam, Bertoli, P., \& Weld, D. S. (2007). A hybridized planner for stochastic domains. In Veloso, M. M., editor, IJCAI, pp. 1972-1978. 
[McCarthy, 1963] McCarthy, J. (1963). Situations, actions, and causal laws. In Minsky, M., editor, Semantic Information Processing, pp. 410-418. MIT Press, Cambridge, MA.

[McDermott, 1998] McDermott, D. (1998). PDDL — the planning domain definition language.

[McMillan, 1992] McMillan, K. L. (1992). Symbolic model checking: an approach to the state explosion problem. In PhD thesis, CMU.

[Müller-Olm et al., 1999] Müller-Olm, M., Schimidt, D., \& Steffen, B. (1999). Model checking: a tutorial introduction. In SAS'99, LNCS 1694, pp. 330-354.

[Murthy et al., 1999] Murthy, S., Akkiraju, R., Goodwin, R., Keskinocak, P., Rachlin, J., Wu, F., Yeh, J., Fuhrer, R., Kumaran, S., Aggarwal, A., Sturzenbecker, M., Jayaraman, R., \& Daigle, R. (1999). Cooperative multiobjective decision support for the paper industry. Interfaces, 29(5):5-30.

[Newell \& Simon, 1963] Newell, A. \& Simon, H. (1963). GPS, a program that simulates human thought. In Feigenbaum, E. \& Feldman, J., Computers and Thought, pp. 279-293. McGrawHill, New York.

[Nicola \& Vaandrager, 1990] Nicola, R. D. \& Vaandrager, F. (1990). Action versus state based logics for transition systems. In Proceedings of the LITP spring school on theoretical computer science on Semantics of systems of concurrent processes, pp. 407-419, New York, NY, USA. Springer-Verlag New York, Inc.

[Nilsson, 1980] Nilsson, N. J. (1980). Principles of artificial intelligence. Tioga, Palo Alto.

[Pecheur \& Raimondi, 2006] Pecheur, C. \& Raimondi, F. (2006). Symbolic model checking of logics with actions. In MoChArt 2006, pp. 1215-1222. Springer Verlag.

[Pednault, 1989] Pednault, E. P. D. (1989). ADL: Exploring the middle ground between strips and the situation calculus. In $K R$, pp. 324-332.

[Pell et al., 1998] Pell, B., Bernard, D. E., Chien, S. A., Gat, E., Muscettola, N., Nayak, P. P., Wagner, M. D., \& Williams, B. C. (1998). An autonomous spacecraft agent prototype. Auton. Robots, 5(1):29-52.

[Pereira \& Barros, 2002] Pereira, S. L. \& Barros, L. N. (2002). High-level robot programs based on abductive event calculus. In CogRob, Cognitive Robotics, Toronto, Canada. (poster). 
[Pereira \& Barros, 2004a] Pereira, S. L. \& Barros, L. N. (2004a). Formalizing planning algorithms: a logical framework for the research on extending the classical planning approach. In ICAPS, Workshop: Connecting Planning Theory with Practice, Canada. (poster).

[Pereira \& Barros, 2004b] Pereira, S. L. \& Barros, L. N. (2004b). High-level robot programming: an abductive approach using event calculus. In SBIA - Advances in Artificial Intelligence, volume 3171 of Lecture Notes in Computer Science, pp. 73-82.

[Pereira \& Barros, 2004c] Pereira, S. L. \& Barros, L. N. (2004c). Planning with abduction: a logical framework to explore extensions to classical planning. In SBIA - Advances in Artificial Intelligence, volume 3171 of Lecture Notes in Computer Science, pp. 62-72.

[Pereira \& Barros, 2007a] Pereira, S. L. \& Barros, L. N. (2007a). Diagramas de decisão binária. RT-MAC-2007-05, IME-USP, São Paulo, Brasil.

[Pereira \& Barros, 2007b] Pereira, S. L. \& Barros, L. N. (2007b). Formalizing planning algorithms for temporally extended goals. RT-MAC-2007-09, IME-USP, São Paulo, Brasil.

[Pereira \& Barros, 2007c] Pereira, S. L. \& Barros, L. N. (2007c). A logic-based agent that plans for extended reachability goals. In Journal of Autonomous Agents and Multi-Agent Systems, Special issue on Computational Logic-based Agents, Canada. (aceito em 15/11/2007).

[Pereira \& Barros, 2007d] Pereira, S. L. \& Barros, L. N. (2007d). A model checker for automated planning with extended reachability goals. In Simpósio Brasileiro de Métodos Formais, Minas Gerais, Brasil. (poster)

[Pereira \& Barros, 2007e] Pereira, S. L. \& Barros, L. N. (2007e). Planejamento baseado em processos de decisão markovianos. RT-MAC-2007-04, IME-USP, São Paulo, Brasil.

[Pereira et al., 2007] Pereira, S. L., Barros, L. N., \& Cozman, F. G. (2007). Strong probabilistic planning. RT-MAC-2007-08, IME-USP, São Paulo, Brasil.

[Pistore et al., 2001] Pistore, M., Bettin, R., \& Traverso, P. (2001). Symbolic techniques for planning with extended goals in non-deterministic domains.

[Pistore \& Traverso, 2001] Pistore, M. \& Traverso, P. (2001). Planning as model checking for extended goals in non-deterministic domains. In IJCAI, pp. 479-486.

[Pnueli, 1977] Pnueli, A. (1977). The temporal logic of programs. In Proc. 18th Symp. Foundations of Computer Science, pp. 46-57. 
[Puterman, 1994] Puterman, M. L. (1994). Markov decision processes—discrete stochastic dynamic programming. John Wiley \& Sons, Inc., NY.

[Ramadge \& Wonham, 1989] Ramadge, P. J. G. \& Wonham, W. M. (1989). The control of discrete event systems. Proceedings of the IEEE, 77(1):81-98.

[Reiter, 2001] Reiter, R. (2001). Knowledge in action - logical foundations for specifying and implementing dynamical systems. MIT Press, Cambridge, MA, USA.

[Rintanen, 2004] Rintanen, J. (2004). Complexity of planning with partial observability. In ICAPS, pp. 345-354.

[Russell \& Norvig, 2002] Russell, S. \& Norvig, P. (2002). Artificial Intelligence: a modern approach, 2nd ed. Prentice-Hall, NJ,USA.

[Saiedian, 1996] Saiedian, H. (1996). An invitation to formal methods. IEEE Computer, 29(4):16-30.

[Schnoebelen, 2003] Schnoebelen, P. (2003). The complexity of temporal logic model checking. In Advances in Modal Logic, papers from 4th Int. Workshop on Advances in Modal Logic (AiML'2002), Sep.-Oct. 2002, Toulouse, France. World Scientific.

[Shanahan, 1997] Shanahan, M. (1997). Solving the frame problem: a mathematical investigation of the common sense law of inertia. MIT Press, Cambridge, MA, USA.

[Simmons et al., 1994] Simmons, R., Krotkov, E., Hebert, M., \& Katragadda, L. (1994). Experience with rover navigation for lunar-like terrains. In Proc. Intl. Lunar Exploration Conf., San Diego, California.

[Taha, 1987] Taha, H. A. (1987). Operations research: an introduction, 4th ed. MacMillan Publishing Co., Inc., Indianapolis, IN, USA.

[Tarski, 1955] Tarski, A. (1955). A lattice-theoretical fixpoint theorem and its applications. In Pacific J. Math., volume 5, pp. 285-309.

[Thiébaux et al., 2006] Thiébaux, S., Gretton, C., Slaney, J., Price, D., \& Kabanza, F. (2006). Decision-theoretic planning with non-markovian rewards. JAIR, 25(2):75-118.

[Trevizan et al., 2007] Trevizan, F. W., Cozman, F. G., \& de Barros, L. N. (2007). Planning under Risk and Knightian Uncertainty. In Veloso, M. M., editor, IJCAI, pp. 2023-2028. 
[Vardi, 1998a] Vardi, M. Y. (1998a). Linear vs. branching time: a complexity-theoretic perspective. In Logic in Computer Science, pp. 394-405.

[Vardi, 1998b] Vardi, M. Y. (1998b). Sometimes and not never re-revisited: on branching versus linear time. In International Conference on Concurrency Theory, pp. 1-17.

[von Neumann \& Morgenstern, 1944] von Neumann, J. \& Morgenstern, O. (1944). Theory of games and economic behavior. Princeton Univertisy Press, New Jersey.

[Weld, 1994] Weld, D. S. (1994). An introduction to least commitment planning. AI Magazine, 15(4):27-61.

[Zilberstein et al., 2002] Zilberstein, S., Washington, R., Bernstein, D. S., \& Mouaddib, A.-I. (2002). Decision-theoretic control of planetary rovers. In Revised Papers from the International Seminar on Advances in Plan-Based Control of Robotic Agents, , pp. 270-289, London, UK. Springer-Verlag.

[Ziller \& Schneider, 2005] Ziller, R. \& Schneider, K. (2005). Combining supervisor synthesis and model checking. Trans. on Embedded Computing Sys., 4(2):331-362. 


\section{Índice Remissivo}

ação, 2

algoritmo

FORTE, 52

FORTECÍCLICO, 55

FrACO, 55

PACTL, 75

PI, 88

PPF, 97

$\mathrm{PPF}^{\prime}, 101$

VACTL, 71

VI, 88

$\mathrm{VI}^{\prime}, 91$

árvore

de computação, 17

de decisão binária, 29

classes

de soluções, 49

condição

alcance, 61

preservação, 61

decisão ótima, 137

diagrama

de decisão binária, 29

domínio

qualitativo, 6

quantitativo, 6 domínio de planejamento

qualitativo, 38

espaço amostral, 132

estado, 2

elementar, 132

estrutura

de Kripke, 16

de execução, 48

evento exógeno, 4

execução

contexto, 60

fator de desconto, 86

fluente, 40

inerte, 40

não-inerte, 40

fórmula

intensão, 70

função

característica, 32

Cobertura, 75

INTENSÃO, 71

INTENSÃOAG, 72

INTENSÃOAU, 73

INTENSÃOEG, 72

INTENSÃOEU, 73

Modelo, 76 


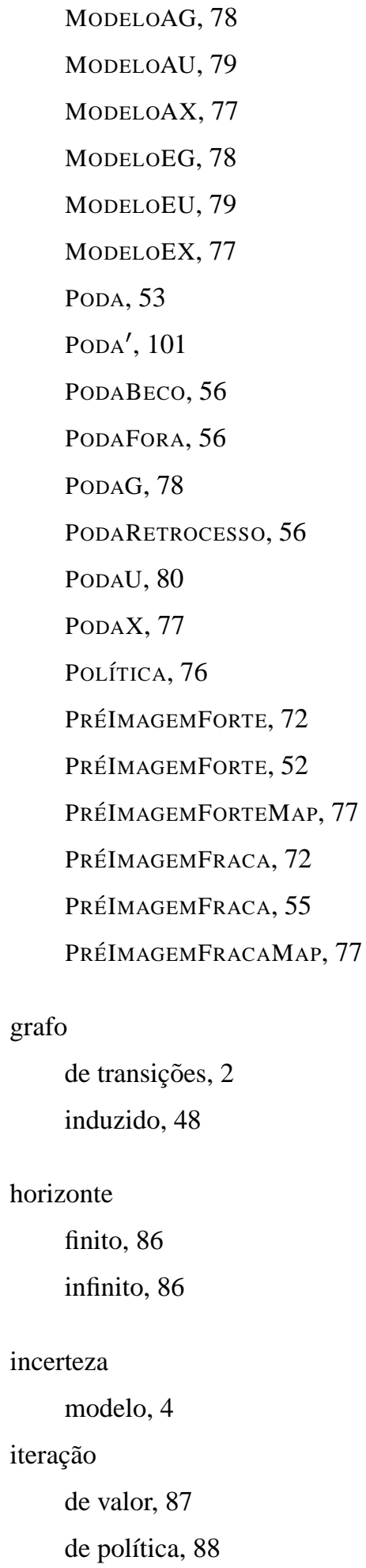

Kolmogorov, axiomas, 132

linguagem

$\mathcal{A R}, 39$

sentenças, 40

lógica

$\alpha$-CTL, 68

modal, 16

temporal, 17

complexidade, 21

CTL, 19

expressividade, 20

LTL, 18

operadores, 18

loterias, 134

meta

alcançabilidade estendida, 61

alcançabilidade simples, 47

estendida, 59

subclasses, 63

modelo

temporal, 16

observabilidade

completa, 5

parcial, 5

planejador, 37

planejamento

clássico, 1

complexidade, 4

domínio, 2 
mundo dos blocos, 2

verificador, 15

problema, 3

solução, 3

política, 7

estacionária, 85

estendida, 60

não-estacionária, 86

ótima, 87

ponto-fixo, 23

preferência

relação, 133

probabilidade

axiomas, 132

condicional, 132

distribuição, 133

incondicional, 132

medidas, 132

produto relacional, 33

teoria

da probabilidade, 131

da utilidade, 133

utilidade

axiomas, 134

esperada, 136

função, 136

princípio, 136

variável

aleatória, 132

verificação

de modelos simbólicos, 26

modelo, 15

problema, 15 